LA W RENCE LIVERMORE NATIONAL LABORATORY
Validation of Numerical Simulations of Explosion Fields

A. L. Kuhl

January 4, 2013 
This document was prepared as an account of work sponsored by an agency of the United States government. Neither the United States government nor Lawrence Livermore National Security, LLC, nor any of their employees makes any warranty, expressed or implied, or assumes any legal liability or responsibility for the accuracy, completeness, or usefulness of any information, apparatus, product, or process disclosed, or represents that its use would not infringe privately owned rights. Reference herein to any specific commercial product, process, or service by trade name, trademark, manufacturer, or otherwise does not necessarily constitute or imply its endorsement, recommendation, or favoring by the United States government or Lawrence Livermore National Security, LLC. The views and opinions of authors expressed herein do not necessarily state or reflect those of the United States government or Lawrence Livermore National Security, LLC, and shall not be used for advertising or product endorsement purposes.

This work performed under the auspices of the U.S. Department of Energy by Lawrence Livermore National Laboratory under Contract DE-AC52-07NA27344. 
DRAFT 8

\title{
Validation of Numerical Simulations of Explosion Fields
}

\author{
Allen L. Kuhl \\ AX Division, WCI \\ Lawrence Livermore National Laboratory \\ 7000 East Avenue \\ Livermore CA 94551
}

\section{LLNL-TR-}

October 16, 2012 



\section{EXECUTIVE SUMMARY}

This report describes test problems that can be used to validate numerical simulations of nuclear explosion fields. A particular emphasis is placed on turbulent mixing and other "non-ideal" effects (thermal layer precursors, turbulent wall jets and dusty boundary layer flows) created by the interaction of the explosion wave with ground surfaces. The importance of such effects is illustrated by a comparison of the peak stagnation pressure versus ground range. In the precursor regime $\left(H O B<700 \mathrm{ft} / K T^{1 / 3}\right.$ and $\left.G R<1100 \mathrm{ft} / K T^{1 / 3}\right)$ stagnation pressures from non-ideal effects are an order of magnitude larger that the corresponding ideal-surface case. Seven problems are considered:

(i) blast waves from nuclear explosions,

(ii) blast wave reflections from ideal surfaces,

(iii) blast wave reflections from ground surfaces,

(iv) spherical mixing layers,

(v) Kelvin-Helmholtz mixing layers,

(vi) wall-bounded mixing layers,

(vii) height of burst curves.

The need for additional work is described in the Recommendations section, which includes tasks in support of the V\&V, Complex Hydro and Output and Effects Programs. 



\section{Table of Contents}

Chapter

page

Introduction

Problem 1: Blast Waves from Nuclear Explosions

1.1 Introduction

1.2 Radiation-hydrodynamics simulation

1.3 Free Air Curves (sea level and US standard atmospheres)

Appendix A: Analytic Solution for a Point Explosion

Appendix B: Analytic Solution for Atmospheric Structure

Problem 2: Blast Wave Reflections from Ideal Surfaces

2.1 Regular reflection from wedge: $M_{s}=2.05, \theta_{w}=60$ degrees, argon, $\gamma=5 / 3$

2.2 Mach reflection from wedge: $M_{s}=2.03, \theta_{w}=27$ degrees, air, $\gamma=1.4$

2.3 Double-Mach reflection from a wedge: $M=8.70, \theta=27$ degrees, air

2.4 Shock reflection factors for air

2.5 Reflection of HE-driven blast wave from a planar surface: $H O B=33.6 \mathrm{~cm} / \mathrm{kg}^{1 / 3}$

2.6 Assessment: reflected pressure versus ground range

Problem 3: Blast Wave Reflections from Ground Surfaces

Appendix A: Empirical thermal layer from Event Priscilla

Appendix B: Color visualization of the precursor wall jet flow

Appendix C: Color visualization of the dusty boundary layer surface

Problem 4: Spherical Mixing Layers

4.1 Visualization

4.2 evolution of the mean profiles and $r m s$ profiles

4.3 evolution of rotational and dilatational TKE spectra

4.4 decay of TKE

Problem 5: Kelvin-Helmholtz Mixing Layers

5.1 Case 1-He over $\mathrm{N}_{2}: U_{1}=1.45 \& U_{2}=0.55 ; \rho_{1}=1 \& \rho_{2}=7 \quad\left(\rho_{i} U_{i}^{2}=Q\right)$

5.2 Case $2-\mathrm{N}_{2}$ over He: $U_{1}=1.45 \& U_{2}=0.55 ; \rho_{1}=7 \& \rho_{2}=1 \quad\left(Q_{1}=49 Q\right)$

5.3 Case $3-\rho=$ const : $U_{1}=1.25 \& U_{2}=0.75 ; \rho_{1}=1 \& \rho_{2}=1$

5.4 Case 4- $\mathrm{N}_{2}$ over air: $U_{1}=1.45 \& U_{2}=0.55 ; \rho_{1}=1 \& \rho_{2}=1 \quad(\rho \cong$ const $)$

Problem 6: Wall Mixing Layers

6.1 dusty boundary layer behind a shock

6.2 dense-gas mixing layer

Problem 7: Height of Burst Curves

7.1 HOB curves from 1-g NITROPENTA charges over ideal surfaces

7.2 HOB curves from 8-lb PBX9404 charges for ideal surfaces

7.3 Nuclear HOB curves for ideal surfaces

Summary and Conclusions

Recommendations

Proposals

References 



\section{Introduction}

This manuscript describes a framework for validating LLNL computer codes for simulating nuclear explosion fields. A particular emphasis is placed on turbulent mixing and "non-ideal" effects ${ }^{1,23}$ resulting from the interaction of an explosion wave with real surfaces. These problems have traditionally proved difficult to validate, even though there appears to be a wealth of information available.

The simplest case is the reflection of a spherical blast wave from an ideal surface, shown in Fig. 1. For the considered height-of-burst $(H O B)$, a simple Mach stem structure (incident shock, Mach stem, reflected shock and slip line $)$ has formed by the time $(0.77 \mathrm{~s})$ depicted in Fig. 1a. This blast-wave-induced Mach stem structure has been visualized by a shadow photograph presented in Fig. 1b.

One of the principal differences between blast waves from nuclear charges versus high explosive (HE) charges is the high temperature in the nuclear fireball $\left(\sim 10^{7} \mathrm{~K}\right)$ versus the $\mathrm{HE}$ fireball $(\sim 3,000 \mathrm{~K})$. The nuclear fireball emits thermal radiation that is absorbed by the ground, forming a high-sound-speed layer on the surface. The reflected shock propagates faster in the hot layer than in the air above it - forming a precursor shock that out-runs the main blast wave, as shown in Fig. 2. Therefore the flow near the surface is dramatically modified compared to the idealsurface case shown in Fig. 1.

If the reflecting surface is actually a ground surface, then the high-speed flow near the surface forms a high-speed wall jet that entrains dust into the flow, forming a turbulent dusty boundary layer (Fig. 3). The flow near the ground is dramatically different than that found in reflections from ideal surfaces (Fig. 1) and hot surfaces (Fig. 2). For example, the peak stagnation pressure for a nuclear $H O B$ is shown in Fig. 4 as a function of ground range $(G R)$. It shows that the peak stagnation pressure for a nuclear $H O B$ can be an order of magnitude larger than that from a blast reflection from an ideal surface. And it is the stagnation pressure that is the relevant parameter for loading above ground structures. This is not taken into account in weapon effects models [1] which are based on ideal-surface $H O B$ curves (see Problem 7).

In this manuscript, we have identified seven problems related to the above-described flow fields:

- Problem 1. Blast Waves from Nuclear Explosions: early-time radiation hydrodynamics flow that evolves into a gasdynamic explosion field. The principal diagnostic is the socalled free air curve (peak over-pressure versus radius from the explosion center).

- Problem 2. Blast Wave Reflections from Ideal Surfaces: regular and Mach reflections from wedges, and the reflection of an HE-driven blast wave from a surface are compared with experimental data.

\footnotetext{
${ }^{1}$ Samuel Glasstone (Editor), Effects of Nuclear Weapons, US Atomic Energy Commission, April 1962, 730 pp.

2 A. L. Kuhl, Airblast Effects from Nuclear Explosions over Real Surfaces, DNUG Meeting, April 2012 (in press).

${ }^{3}$ A. L. Kuhl, P. Wang, Nuclear Explosion at 500 feet above a Ground Surface, NECDC 2012 Proceedings (in press)
} 


\section{Introduction}

- Problem 3. Blast Wave Reflections from Ground Surfaces: precursor shock structure, turbulent wall jet and dusty boundary layer flow.

- Problem 4. Spherical Mixing Layers: evolution of the turbulent flow, mixing layer growth, mean and root-mean-squared $(R M S)$ profiles in the mixing layer, decay of the turbulent kinetic energy, evolution of the turbulent kinetic energy spectra (both rotational and dilatational components).

- Problem 5. Kelvin-Helmholz Mixing Layers: transition in free shear layers are compared with the fundamental experiments of Brown \& Roshko (1974) and Oster \& Wignanski (1982).

- Problem 6. Shock-induced Wall Mixing Layers: transition in dusty boundary layers and dense-gas boundary layers is compared with experiments.

- Problem 7. Height of Burst Curves: $H O B$ curves for HE-driven blast wave reflections from smooth versus ideal surfaces (Reichenbach \& Kuhl, 1991) are compared with nuclear $H O B$ curves for ideal surfaces (Carpenter, 1994).

We hope that this manuscript will serve as benchmark for validating hydro-code simulations of nuclear explosions and their reflections from various surfaces. 


\section{Introduction}

(a) Numerical Simulation $(t=0.77 \mathrm{~s})^{3}$

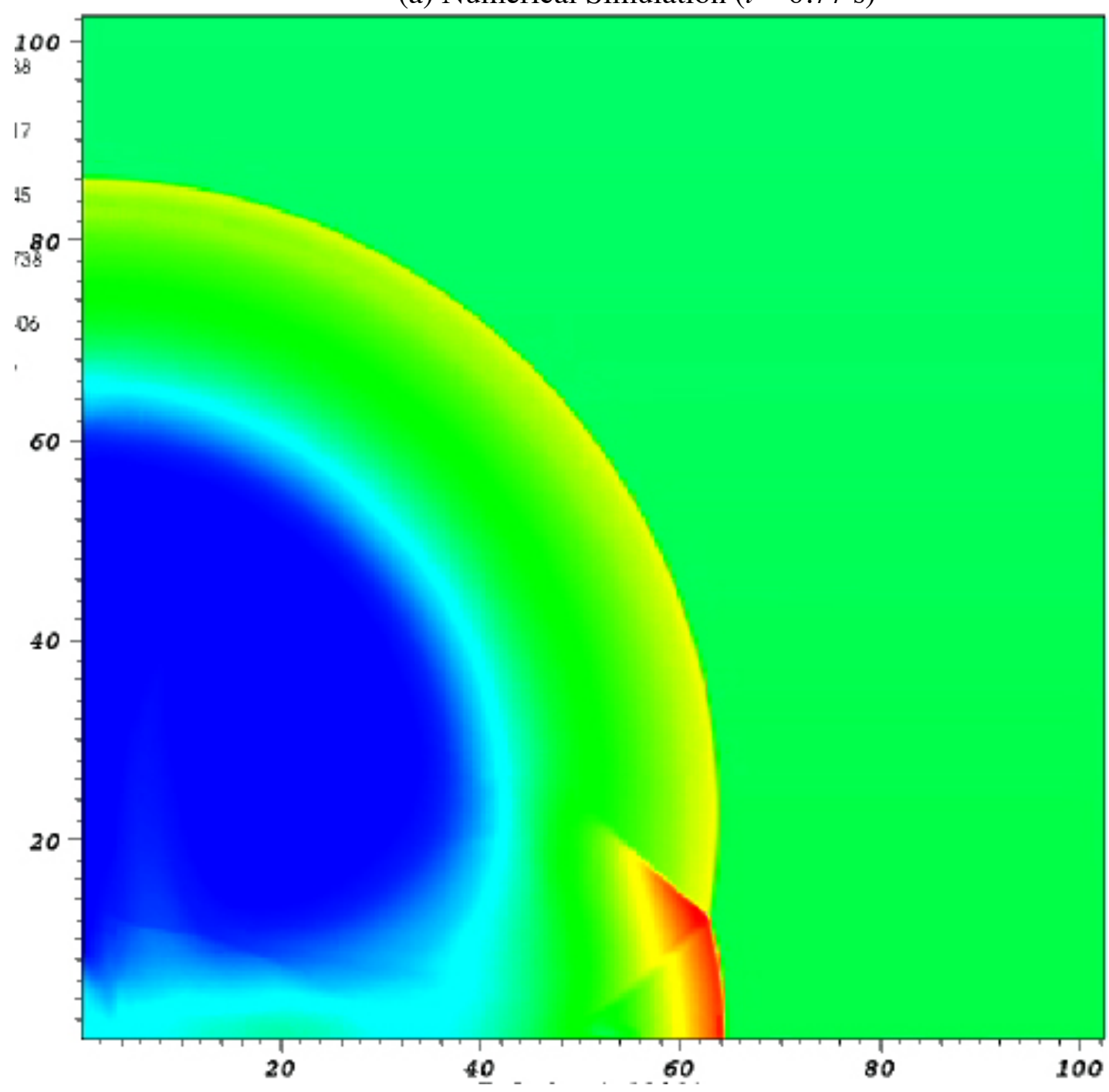

(b) Shadow Photograph (courtesy of EMI)

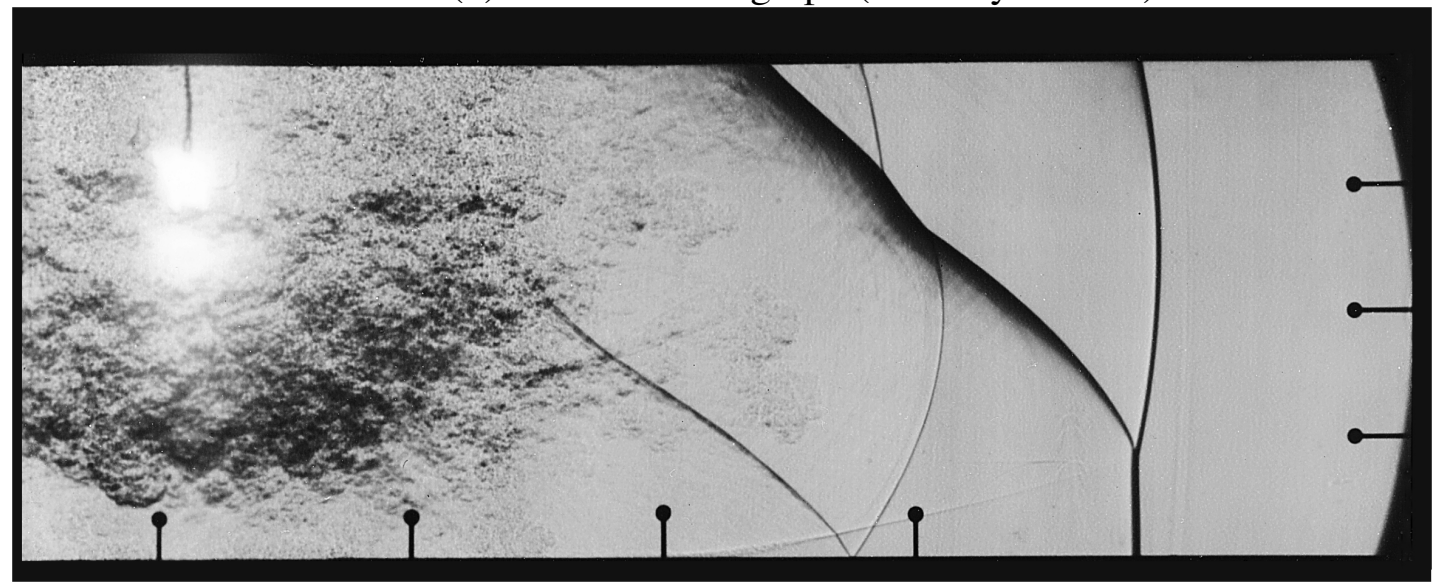

Figure 1. Blast reflection from an ideal surface. 


\section{Introduction}

(a) Numerical Simulation ${ }^{3}$

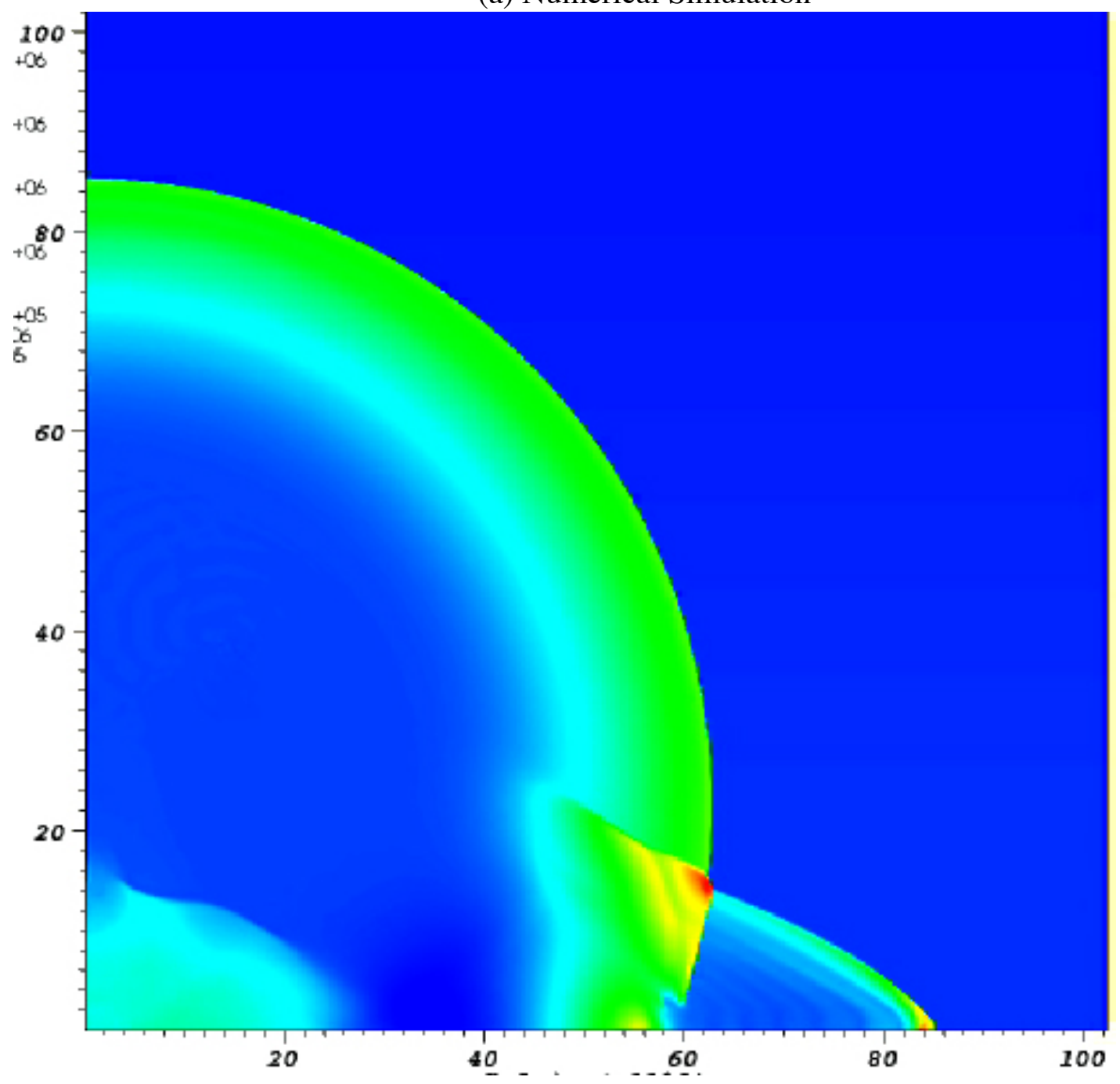

(b) Shadow Photograph (courtesy of EMI)

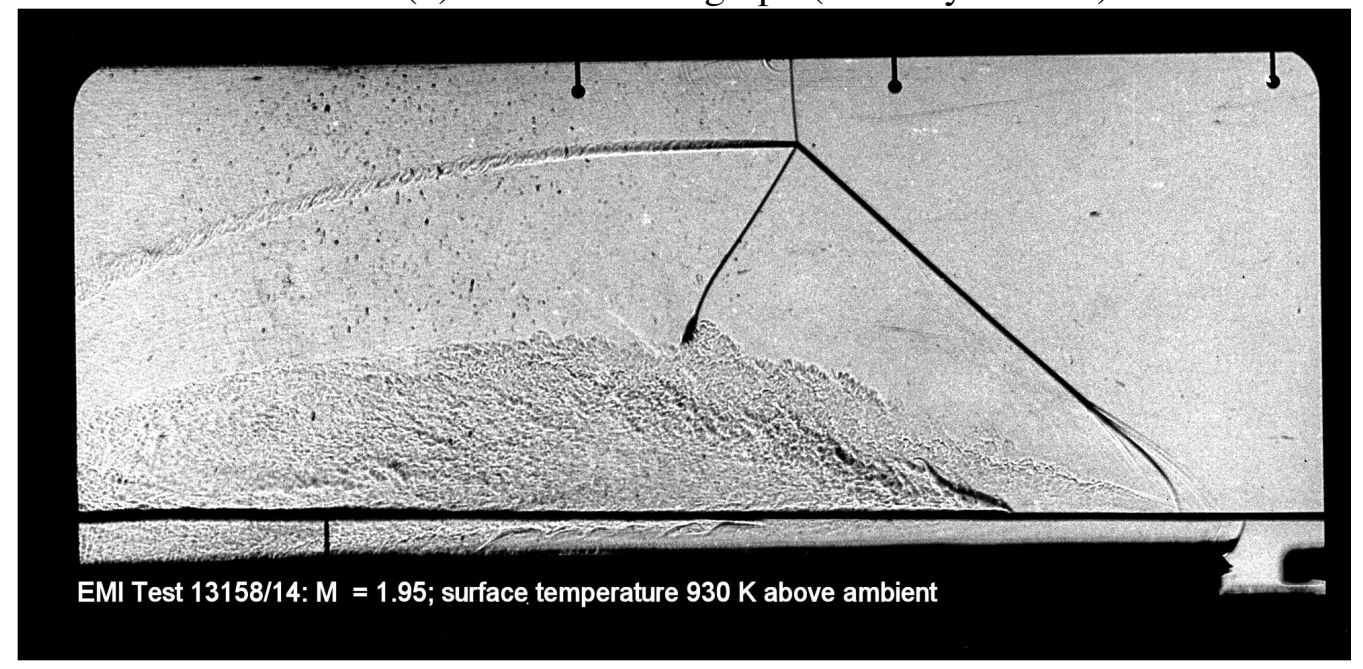

Figure 2. Blast wave reflection from a hot surface. 


\section{Introduction}

(a) Numerical Simulation ${ }^{3}$

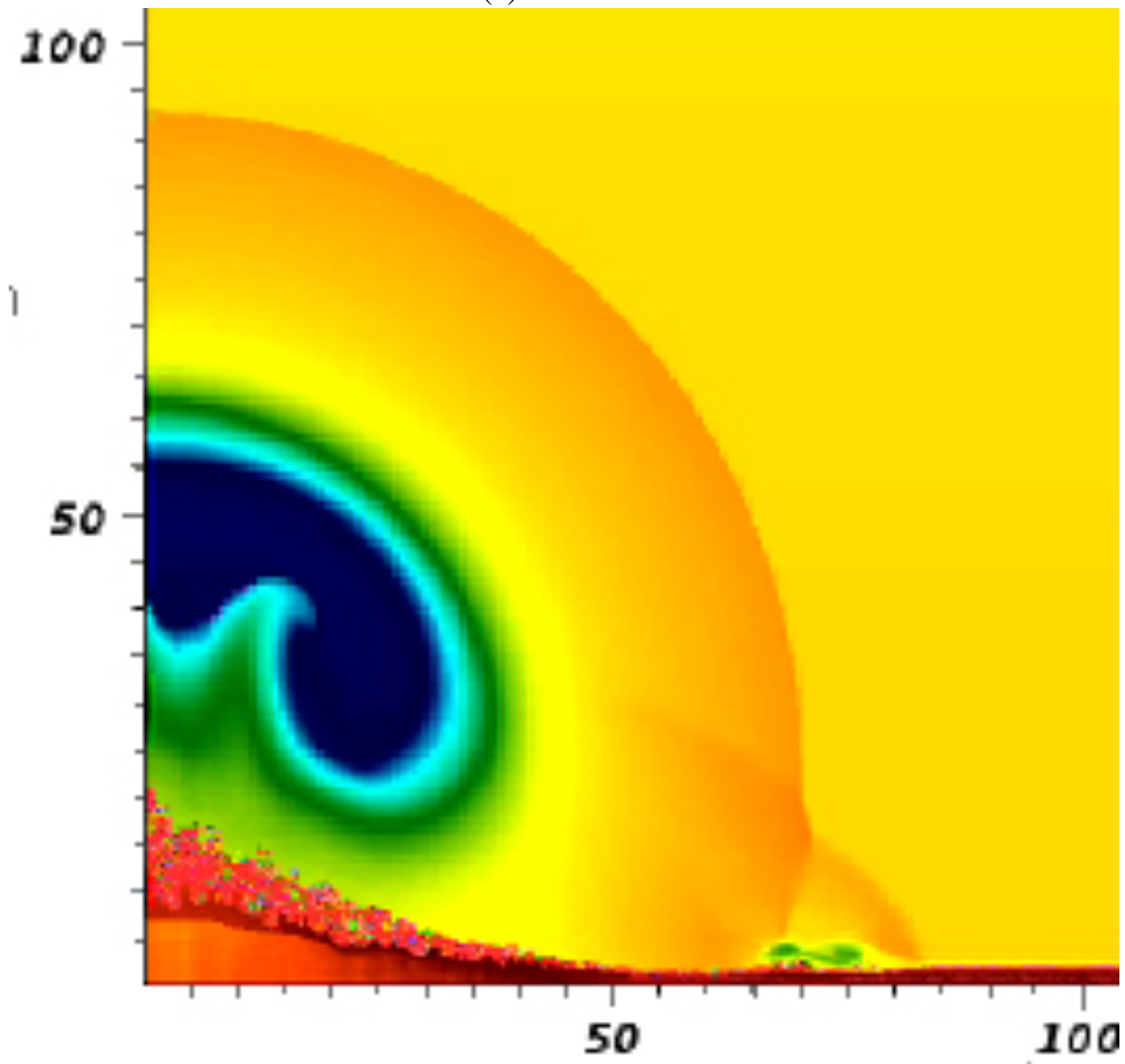

(b) Shadow Photograph (courtesy of EMI)

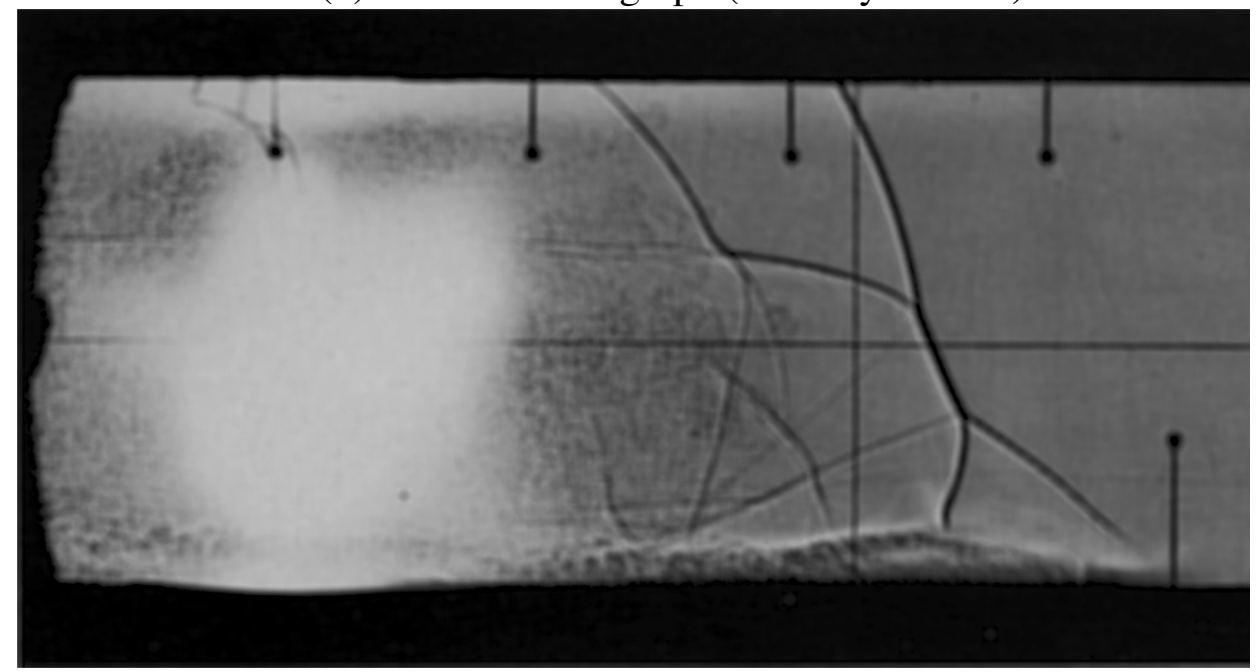

Figure 3. Blast wave reflection from a ground surface. 


\section{Introduction}

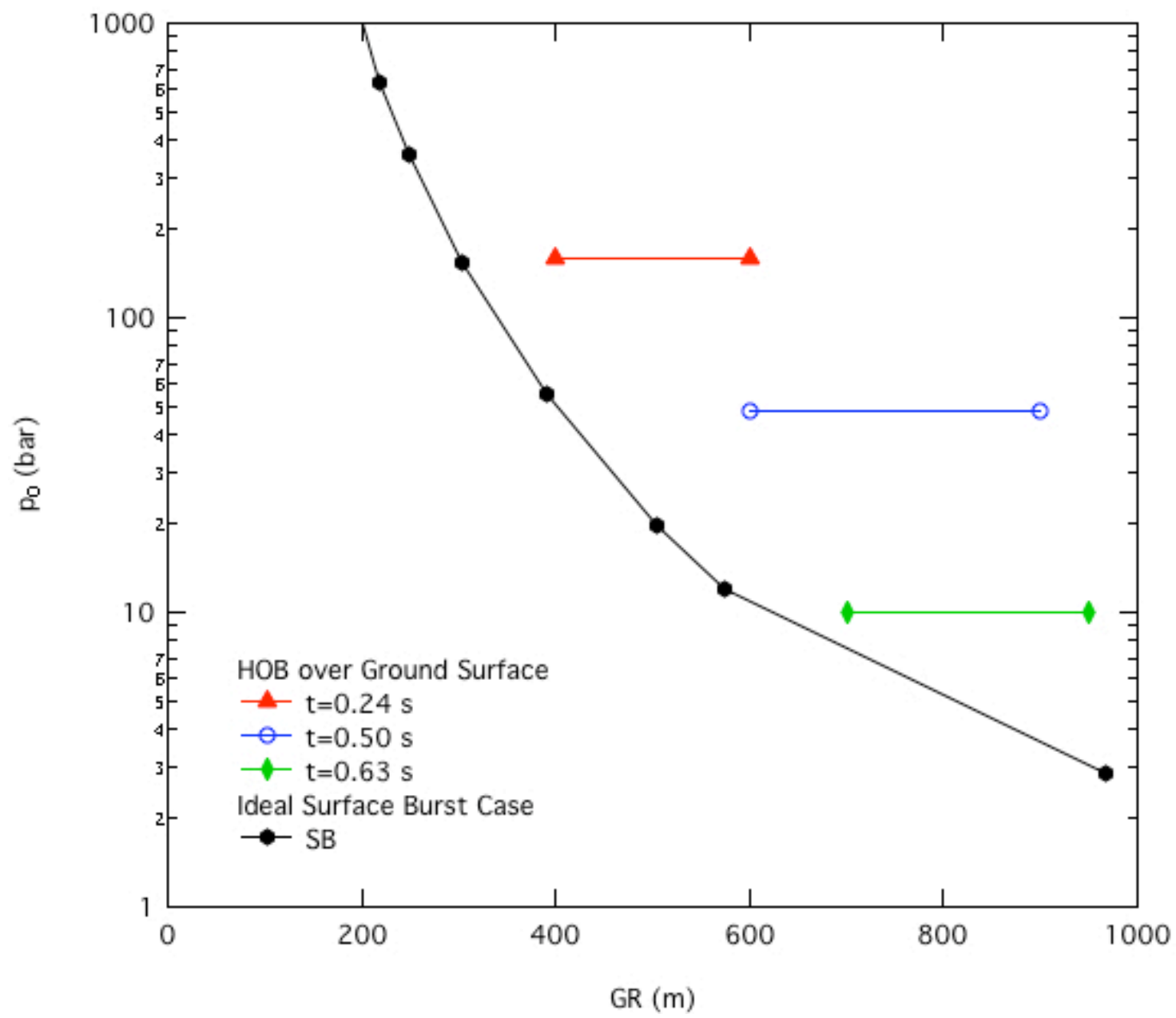

Figure 4. The peak stagnation pressure $\left(p_{0}\right)$ as a function of ground range (GR) for a height of burst (HOB) explosion is compared with that from an ideal surface burst ${ }^{3}$. The airblastprecursor-induced wall jet increases the stagnation pressure on above-ground structures by about factor of 10 , compared to the ideal surface case (for $G R<1000 m$ ). This "non-ideal" effect is not taken into account in previous studies. 


\section{Problem 1: Blast Waves from Nuclear Explosions}

\section{Introduction}

The theoretical description of blast waves from nuclear charges has been predicted by Hal Brode using a one-dimensional Lagrangian radiation-hydrodynamics code with a von Neumann artificial viscosity to capture the shock [1]-[4], [8]-[9]. The solution was scaled to 1 kiloton using cube-root scaling and published in the Annual Reviews of Nuclear Science in 1968. Much of that article was used by Samuel Glasstone in his famous monograph: Review of Nuclear Weapons Effects [5] published in 1962, and by Philip J. Dolan, Editor of Effects Manual 1 (EM-1) Part I: Phenomenology [22] published by the Defense Nuclear Agency in 1972.

The flow field from a nuclear explosion is quite accurately described by the "point explosion solution", first discovered by Academician Leonid Ivanovich Sedov [16-17] in 1946, and independently by Sir Geoffrey I. Taylor [12-13] in 1950. This is one of the prominent similarity solutions found in Sedov's seminal monograph: Similarity and Dimensional Analysis in Mechanics - the definitive work on this subject. Its mathematical underpinnings were provided by Bridgeman's famous book: Dimensional Analysis published in 1922. This method was used by Oppenheim et al. [11] to predict all possible self-similar blast waves bounded by a strong shock in 1972. Other similarity solutions can be found in Stanyukovich's book: Unsteady Motion of Continuous Media [18-19] published in 1960.

The point-source solution was extended to low pressures by John von Neumann in 1941. Shock tracking was used to accurately capture the shock jump conditions in the 1D Lagrangian simulation [14]. Originally this manuscript (NDRC Div. B, Report AM-9, 1941) was classified; in 1955 it was published under the title "Blast Wave Calculation" [15].

\section{Radiation Hydrodynamics Solution}

The formation of a blast wave from a nuclear explosion is illustrated in Fig. 1. The time-distance diagram shows the propagation and decay of the radiation front and the case shock formed by the expanding bomb vapors. The case shock overtakes the radiation front at $t=0.1 \mathrm{~ms} / \mathrm{KT}^{1 / 3}$ and $R=8 \mathrm{~m} / K T^{1 / 3}$; thereafter the flow field is accurately represented by the point source solution, as shown in Fig. 2. The fireball diameter from the nuclear simulation is about 10 percent bigger due to radiation-diffusion growth, over and above the hydrodynamic growth.

\section{Free Air Curves ${ }^{4}$}

Computed points from Brode's kiloton (KT) and megaton (MT) rad-hydro simulations are shown in Fig. 3. Notice that the kiloton results (blue points) and MT results (red points) lie on a single composite curve:

$$
\Delta p \sim 2.6+3.5 \times 10^{8} / R^{2.7}
$$

\footnotetext{
${ }^{4}$ i.e., the peak over-pressure as a function of scaled radius from the explosion center.
} 


\section{Problem 1. Blast Waves from Nuclear Explosions}

The blast wave from a 36.6 KT explosion was simulated with our two-dimensional (2D) AMR code. A second order Godunov scheme was used to integrate the gas dynamic conservation laws. A tabulated equation of state, based on Gilmore's thermodynamic solution for the equilibrium properties of air up to $10^{7} \mathrm{~K}$ [6], was used. Two cases were computed: (i) a 2D explosion in a uniform sea level atmosphere, and (ii) a 2D explosion in a stratified US Standard atmosphere (see Appendix B for its definition). The point explosion solution (Fig. A2) was used to initialize the grid, starting when the shock radius was $160 \mathrm{ft} / \mathrm{KT}^{1 / 3}$. Results were cube root scaled to one kiloton, and presented in Fig. 4. Computed peak shock pressures for the uniform-atmosphere case (black points) lie on top of the Brode's free air curve. This proves that outside $30 \mathrm{ft} / K T^{1 / 3}$, the blast wave solution is totally gasdynamic (i.e., radiation diffusion no longer influences the solution). Also shown in Fig. 4 are the results from the 2D simulation of an explosion in the US standard atmosphere (green points), sampled along a horizontal radius at the burst height. One can see that the green points fall further and further below the free air curve as the blast wave expands. This is because the pressure gradient in the atmosphere induces a rarefaction in the blast wave, causing the shock to weaken when measured in the horizontal plane.

\section{References}

[1] H. L. Brode, Numerical solutions of spherical blast waves, J. App. Phys. 16 (6) 1955, pp. 766-775. (Evolution of blast wave from point-source solution: 2 kilo-bars to 0.1 bar regime)

[2] H. L. Brode, Theoretical Description of the Blast and Fireball for a Sea-Level Megaton Explosion, RM-2248, Rand Corp. 1959, 147 pp.

[3] H. L. Brode, Theoretical Description of the Blast and Fireball for a Sea-Level Kiloton Explosion, RM-2246, 1966 (FOUO).

[4] H. L. Brode, Review of Nuclear Weapons Effects, Annual Reviews of Nuclear Science vol. 18, 1968, pp. 153-202.

[5] S. Glasstone (Ed.) The Effects of Nuclear Weapons, US Atomic Energy Commission, 1962, 730 pp.

[6] F. R. Gilmore, Equilibrium Composition and Thermodynamic Properties of Air to 24,000 K, Rand Corp. Report RM-1543, 1955, 68 pp; also see DASA 1971-1 THERMAL RADIATION PHENOMENA, J. L. Magee and H. Aroeste, Eds. 3-27-67-1 Vol. 1, May 1967, 333 pp. (vid. esp. Chapter 1: The Equilibrium Thermodynamic Properties of High Temperature Air by F. R. Gilmore).

[7] H. L. Brode, Blast wave from a spherical charge, Physics of Fluids 2 (2) 1959, 217-229; also Calculation of the Blast Wave from a Spherical Charge of TNT, Rand Corp. Report \# RM-1965, 1957, $1-68$.

[8] R. K. M. Landshoff, Ed., Thermal Radiation Phenomena; Volume 5: H. L. Brode and R. K. M. Landshoff Radiation Hydrodynamics of High Temperature Air, DASA 1917-5, Lockheed Missiles and Space Company, 1967, 172 pp. (vid. esp. Chapter 2: Physics of Fireballs, pp. 36-58, one kiloton at sea level conditions).

[9] H. L. Brode, Gasdynamic motion with radiation: a general numerical method, Astronautica ACTA vol. 14 (5), 1969, pp. 443-444.

[10] M. Dubin, N. Sissenwine, H. Wexler, cochairmen, US Standard Atmosphere 1962, US Government Printing Office, Washington, DC 1962, 278 pp.

[11] A. K. Oppenheim, A. L. Kuhl, E. A. Lundstrom, M. M. Kamel, A parametric study of self-similar blast waves, J. Fluid Mech. 52(4), 1972, pp. 657-682.

[12] Taylor, G. I., "The Formation of a Blast Wave by a Very Intense Explosion, Part 1: Theoretical Discussion," first published in British Report RC-210, 1941; revised version in Proc. Royal Society of London Series A, Vol. 201, 1950, pp. 159-174.

[13] Taylor, G. I., "The Formation of a Blast Wave by a Very Intense Explosion, Part 2: The Atomic 


\section{Problem 1. Blast Waves from Nuclear Explosions}

Explosion of 1945" Proc. Royal Society of London Series A, Vol. 201, 1950, pp 175-186.

[14] von Neumann, J. "The Point Source Solution," first published in NDRC Div. B. Report AM-9, 1941; then in Shock Hydrodynamics and Blast Waves, Bethe, H. A., ed., AECD-2860, 1944; revised version in Blast Waves, Bethe, H. A., ed. , Los Alamos Scientific Laboratory Report LA-2000, 1947, pp. 2755; reprinted in John von Neumann Collected Works, Taub, A. H, ed., Vol. VI, Pergamon Press, New York 1963, pp. 219-237.

[15] von Neumann, J., and Goldstine, H., "Blast Wave Calculation," Comm. Pure and Applied Mathematics Vol. 8, 1955, pp. 327-353; reprinted in John von Neumann Collected Works, Taub, A. M., ed., Vol. VI, Pergamon Press , New York, 1963 , pp . 386-412.

[16] Sedov, L. I., "The Motion of Air due to an Intense Explosion" Doklady AN SSSR, Vol. 52, No. 1, 1946, pp 17-20.

[17] Sedov, L. I., "Rasprostraneniya sil'nykh vzryvnykh voln" (The Propagation of Intense Explosion Waves), Prikladnaya Matematika i Mekhanika, Vol. 10, No 2, 1946, pp. 241-250.

[18] Stanyukovich. K. P., "Application of Particular Solutions for Equations of Gas Dynamics to the Study of Blast and Shock Waves," Report of the Academy of Science of the USSR, Vol. 52, No. 7, 1946.

[19] Stanyukovich. K. P., Unsteady Motion of Continuous Media, Gostekhizdat, Moscow, 1955; English translation. Holt, M., ed., Pergamon Press, New York, 1960, 745 pp.

[20] P. W. Bridgeman, Dimensional Analysis, Yale University Press, 1922, 113 pp.

[21] Sedov, L. I., Similarity and Dimensional Method in Mechanics, Friedman, M., translator, Holt, H., ed., 4th Printing, Academic Press, New York, 1959, (vid. sp. "Chapter IV: One-dimensional Unsteady Motion of a Gas"); orginially published as Methody teorii razmernostey I teorii podobiyav mekhanike (Methods of Dimensional Theory and Similarity Theory in Mechanics), MoscowLeningrad, Gostekhizdat, 1944, 136 pp.

[22] P. J. Dolan, Editor, Capabilities of Nuclear Weapons, DNA EM-1 Part I: Phenomenology, Defense Nuclear Agency, Washington, DC 1972 (revised 1 August 1981).

[23] A. L. Kuhl, P. Wang, Nuclear Explosion at 500 feet above a Ground Surface, NECDC 2012 Proceedings (in press)

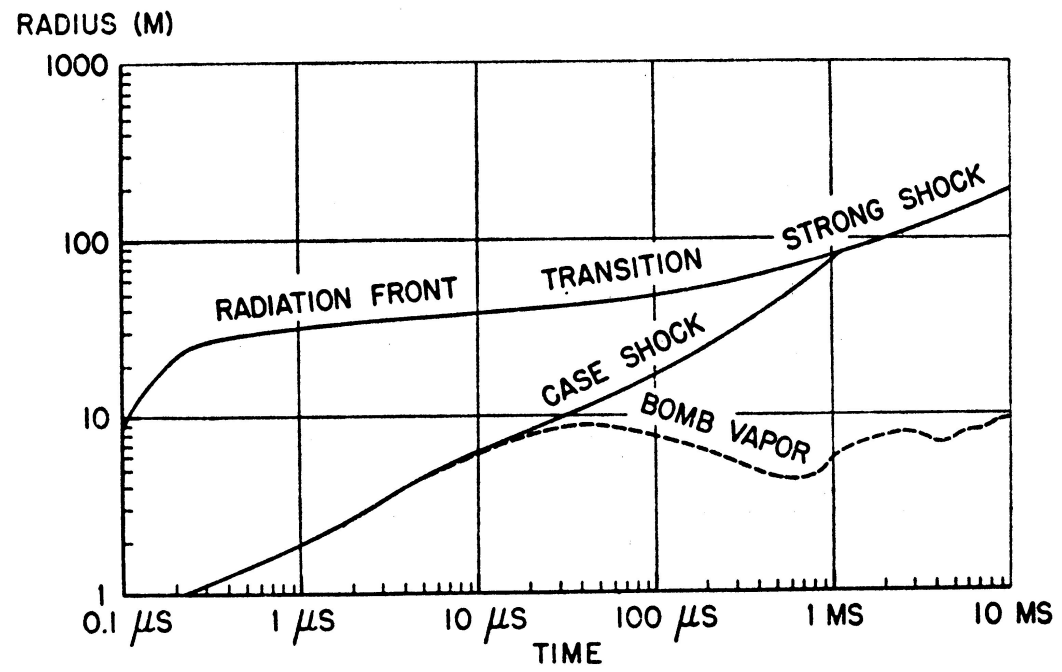

Figure 1.1 Time-distance diagram of the evolution of the thermal wave front and bomb case shock for a 1-MT explosion, based on Brode's 1D numerical simulations [4]. 


\section{Problem 1. Blast Waves from Nuclear Explosions}
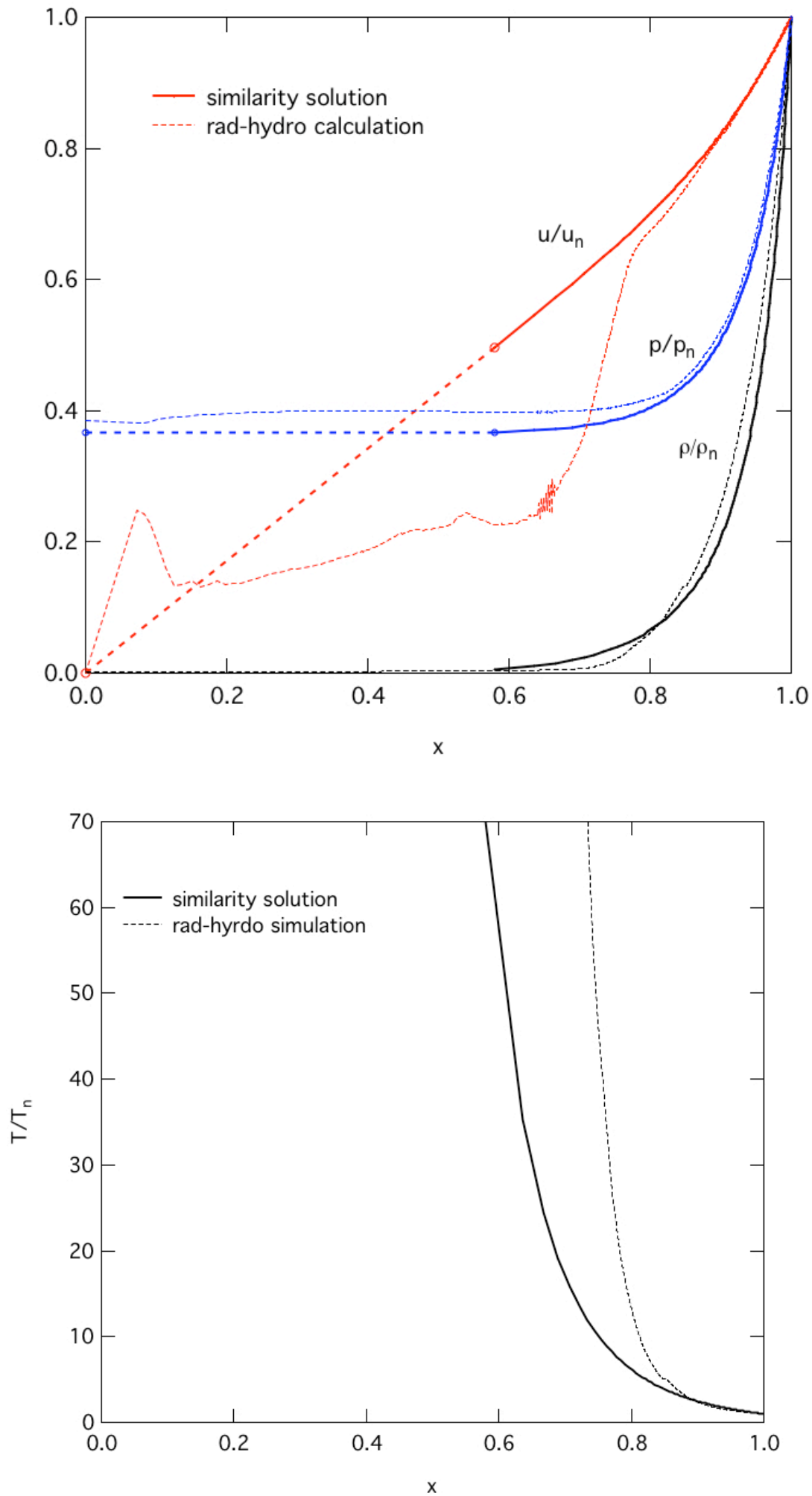

Figure 1.2. Comparison of the rad-hydro simulation with the similarity solution (Appendix 1). 
Problem 1. Blast Waves from Nuclear Explosions

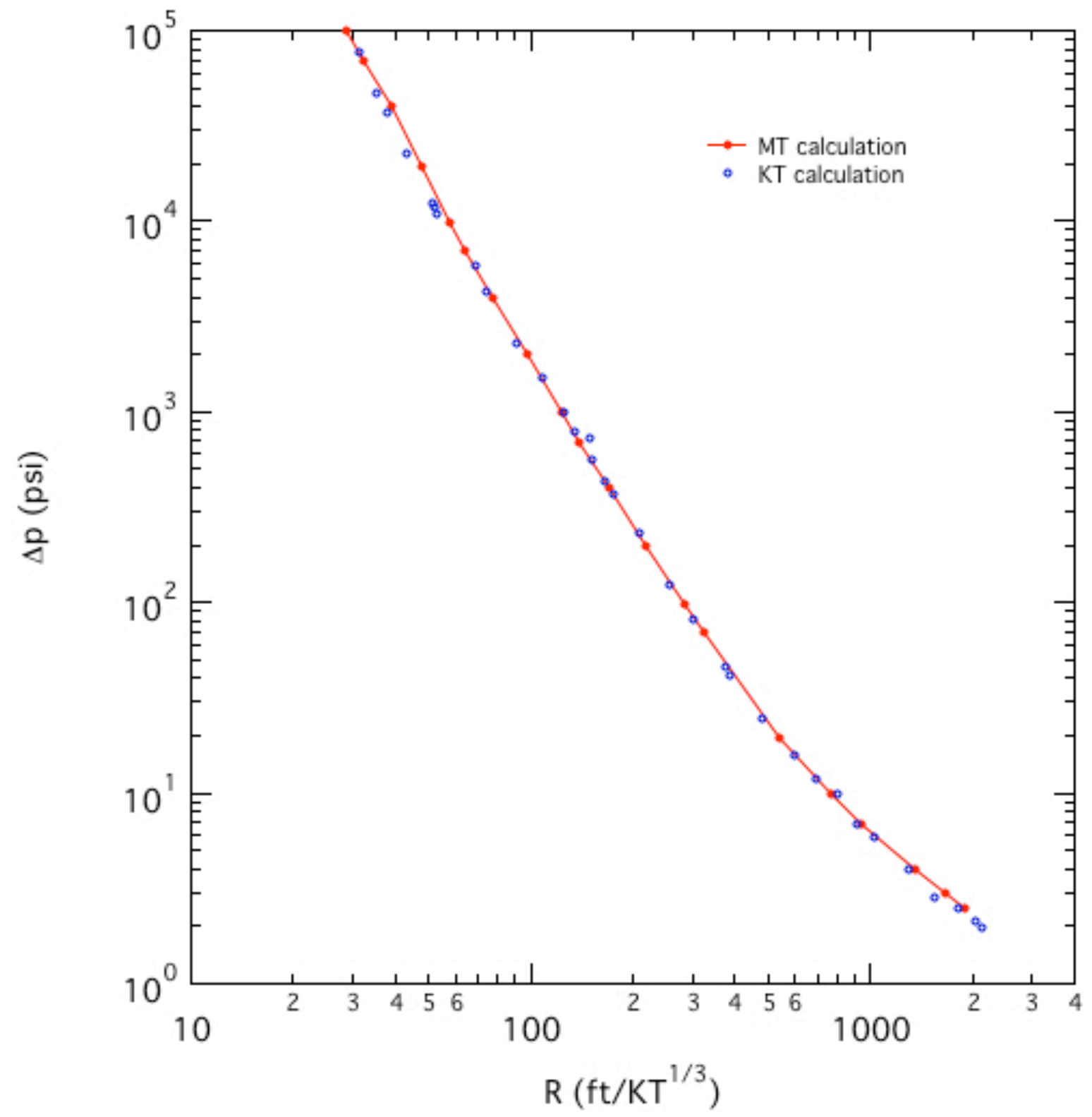

Figure 1.3 Theoretical free-air curve for nuclear explosions in a sea-level atmosphere, as computed by Brode. ${ }^{[1]}$ 
Problem 1. Blast Waves from Nuclear Explosions

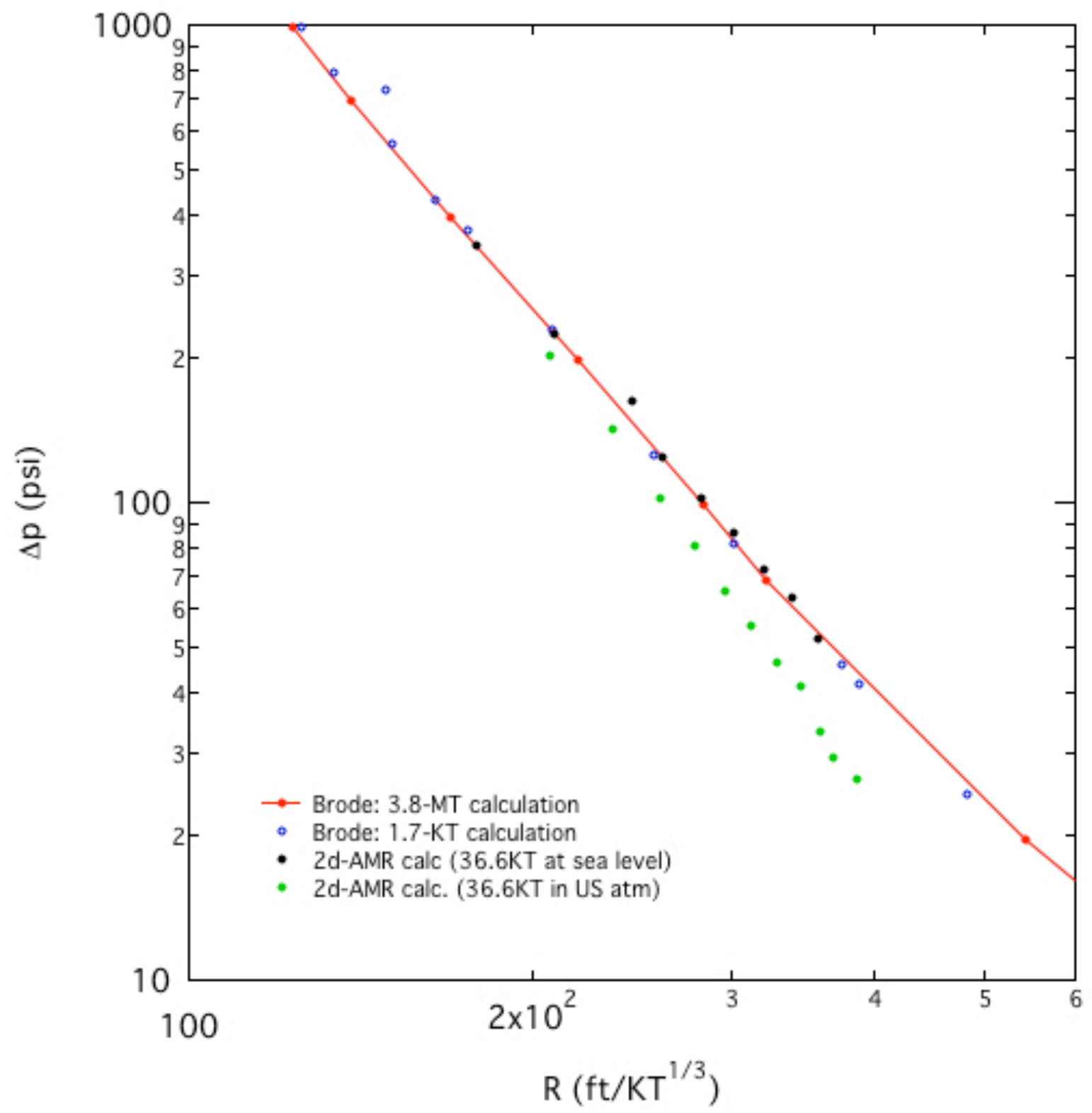

Figure 1.4 Comparison of the pressure-range curves from AMR code simulations of $36.6 \mathrm{KT}$ nuclear explosions in a sea level atmosphere (black dots) ${ }^{23}$ and a US standard atmosphere (green dots $)^{23}$ with the free air curve of Brode. 


\section{Problem 1. Blast Waves from Nuclear Explosions}

\section{Appendix A. Analytic Solution for a Point Explosion ${ }^{5}$}

1. Integral Curve: $Z(F)=\frac{\gamma-1}{2} \frac{(1-F) F^{2}}{F-1 / \gamma} \quad 1 / \gamma \leq F \leq 2 /(\gamma+1)$

2. Quadrature: $\quad x(F)=\left(\frac{F}{F_{n}}\right)^{\frac{-2}{\lambda+2}}\left(\frac{F-1 / \gamma}{F_{n}-1 / \gamma}\right)^{d_{1}}\left(\frac{c-F}{c-F_{n}}\right)^{d_{2}}$

where

$$
\begin{aligned}
& \lambda=j+1=3 \\
& \alpha=(j+1)(\gamma-1)+2=3 \gamma-1=3.2 \\
& c=(\lambda+2) / \alpha=5 / \alpha=5 /(3 \gamma-1)=1.56250 \\
& d_{1}=\frac{\gamma-1}{\alpha(\gamma c-1)}=0.10526 \\
& d_{2}=-\frac{2+(\gamma+1)(\gamma c-2) c}{\alpha c(\gamma c-1)}=-0.40592 \\
& F_{n}=\frac{2}{\gamma+1}=0.83333 \\
& Z_{n}=\gamma F_{n}\left(1-F_{n}\right)=0.1944
\end{aligned}
$$

\section{Flow Field:}

Velocity: $\quad \frac{u(x)}{u_{n}}=x \frac{F}{F_{n}}$

Density: $\quad \frac{\rho(x)}{\rho_{n}}=\left[\left(\frac{Z}{Z_{n}}\right)\left(\frac{1-F}{1-F_{n}}\right) x^{5}\right]^{\frac{1}{(\gamma-2)}}$

Temperature: $\quad \frac{T(x)}{T_{n}}=x^{2} \frac{Z}{Z_{n}}$

Pressure: $\quad \frac{p(x)}{p_{n}}=\frac{\rho}{\rho_{n}} \frac{T}{T_{n}}$

\footnotetext{
${ }^{5}$ A. K. Oppenheim, A. L. Kuhl, E. A. Lundstrom, M. M. Kamel, A parametric study of self-similar blast waves, $J$. Fluid Mech. 5(4), 1972, pp. 657-682.
} 
Problem 1. Blast Waves from Nuclear Explosions

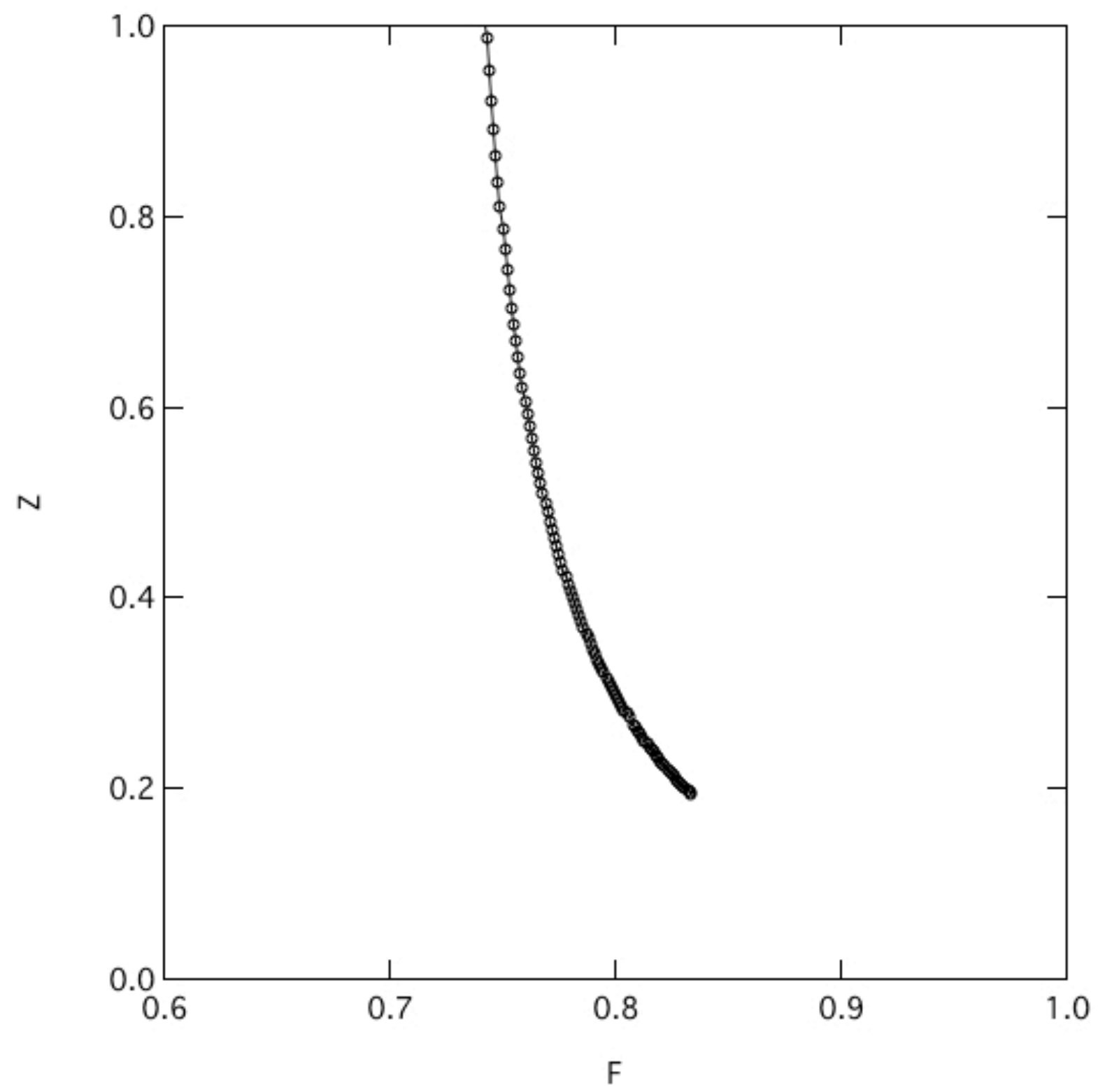

Figure A-1. The Z-F phase plane for analysis of self-similar blast waves $(j=2, \gamma=1.4)$. 
Problem 1. Blast Waves from Nuclear Explosions
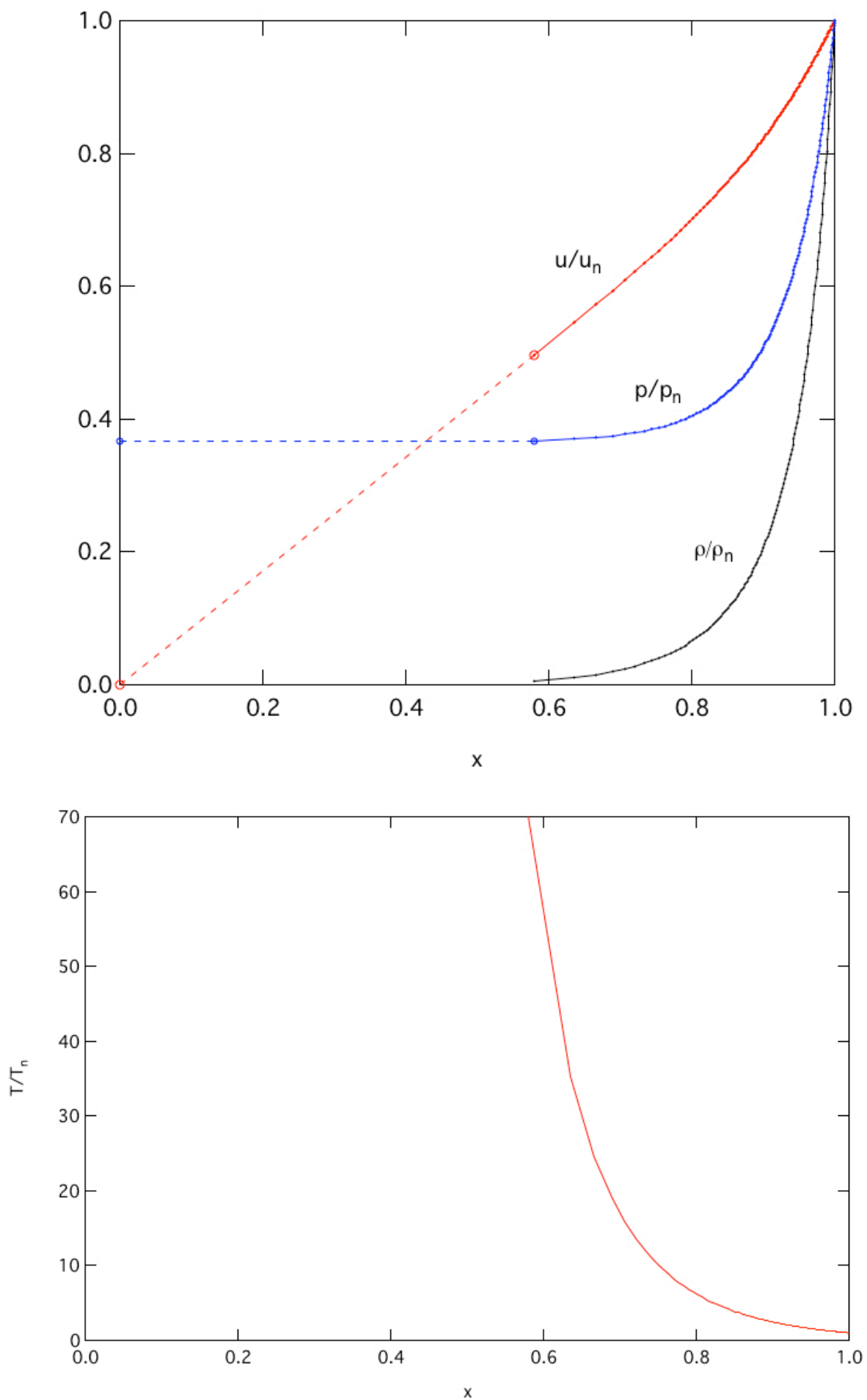

Figure A-2. Similarity solution for a point explosion blast wave $(\gamma=1.4)$. 


\section{Problem 1. Blast Waves from Nuclear Explosions}

\section{Appendix B: Analytic Solution for Atmospheric Structure ${ }^{6}$}

Hydrostatic Law:

$$
d p=-\rho(z) g(z) d z
$$

Perfect Gas Law: $\quad \rho=p \frac{M_{0}}{R_{u}} \frac{1}{T}$

Empirical Temperature Profile: $\quad T(H)=T_{b}+L_{b}^{\prime}\left(H-H_{b}\right)$

Geo-potential Height: $\quad h=H-H_{b}=\int_{H_{b}}^{H} d H=\int \frac{g(z)}{g_{0}} d z$

Pressure:

$$
\begin{aligned}
& d p=-p \frac{M_{0}}{R_{u}} \frac{g(z) d z}{T(z)}=-p \frac{g_{0} M_{0}}{R_{u}} \frac{g(z) d z}{g_{0} T(z)} \\
& \int d \log p=-\frac{g_{0} M_{0}}{R_{u}} \int \frac{d H}{T(H)}=-\frac{g_{0} M_{0}}{R_{u}} \int \frac{d H}{T_{b}+L_{b}^{\prime} H} \\
& \log _{e}\left(p / p_{b}\right)=-\frac{g_{0} M_{0}}{R_{u}} \log _{e}\left(\frac{T_{b}+L_{b}^{\prime}\left(H-H_{b}\right)}{T_{b}}\right)
\end{aligned}
$$

Power Law $\left(L_{b}^{\prime} \neq 0\right): \quad \quad \frac{p}{p_{b}}=\left(\frac{T_{b}}{T_{b}+L_{b}^{\prime} h}\right)^{\frac{g_{0} M_{0}}{R_{u} L_{b}^{\prime}}}$

Exponential Law $\left(L_{b}^{\prime}=0\right): \quad \frac{p}{p_{b}}=\exp \left(-\frac{g_{0} M_{0}}{R_{u} T_{b}} h\right)$

Altitude Regime $0<H<11 \mathrm{~km}$ :

$$
\frac{p}{p_{o}}=\left(\frac{288.15-6.5 h}{288.15}\right)^{C_{1}}
$$

Altitude Regime $11 \mathrm{~km}<H<20 \mathrm{~km}: \frac{p}{p_{1}}=\exp \left(-C_{2} h\right)$

Density:

$$
\frac{\rho(H)}{\rho_{0}}=\frac{p(H)}{p_{0}} \times \frac{T_{0}}{T(H)}
$$

where $h \equiv H-H_{b}(k m)$

\footnotetext{
${ }^{6}$ M. Dubin, N. Sissenwine, H. Wexler, Co-chairmen, US Standard Atmosphere 1962, US Government Printing Office, Washington, DC 1962, 278 pp. (produced by the United States Committee on Extension to the Standard Atmosphere: COESA)
} 


\section{Problem 1. Blast Waves from Nuclear Explosions}

Evaluation of Constants

$$
\begin{aligned}
& C_{1}=-\frac{g_{0} M_{0}}{R_{u} L_{b}^{\prime}}=\frac{980.665\left(\mathrm{~cm} / \mathrm{s}^{2}\right) \times 28.9644(\mathrm{~g} / \mathrm{mol})}{8.31432 \times 10^{7}(\mathrm{dyn}-\mathrm{cm} / \mathrm{mol}-\mathrm{K}) \times 6.5 \mathrm{~K} / 10^{5} \mathrm{~cm}}=5.255822518 \\
& C_{2}=\frac{g_{0} M_{0}}{R_{u} T_{1}}=\frac{980.665\left(\mathrm{~cm} / \mathrm{s}^{2}\right) \times 28.9644(\mathrm{~g} / \mathrm{mol})}{8.31432 \times 10^{7}(\mathrm{dyn}-\mathrm{cm} / \mathrm{mol}-\mathrm{K}) \times 216.65(\mathrm{~K})}=0.157688413 / \mathrm{km} \\
& p_{0}=1.325 \times 10^{6} \text { dynes } / \mathrm{cm}^{2} \& T_{0}=288.15 \mathrm{~K} @ \text { sea level } ; L_{1}^{\prime}=-6.5 \mathrm{~K} / \mathrm{km} \\
& p_{1}=0.223361 \times p_{0} \& T_{1}=216.65 \mathrm{~K} @ 11 \mathrm{~km} ; L_{2}^{\prime}=0 \\
& g_{0}=980.665 \mathrm{~cm} / \mathrm{s}^{2} \\
& M_{0}=28.9644 \mathrm{~g} / \mathrm{mol} \\
& R_{u}=8.31432 \text { joules } / \mathrm{K}-\mathrm{mol}
\end{aligned}
$$

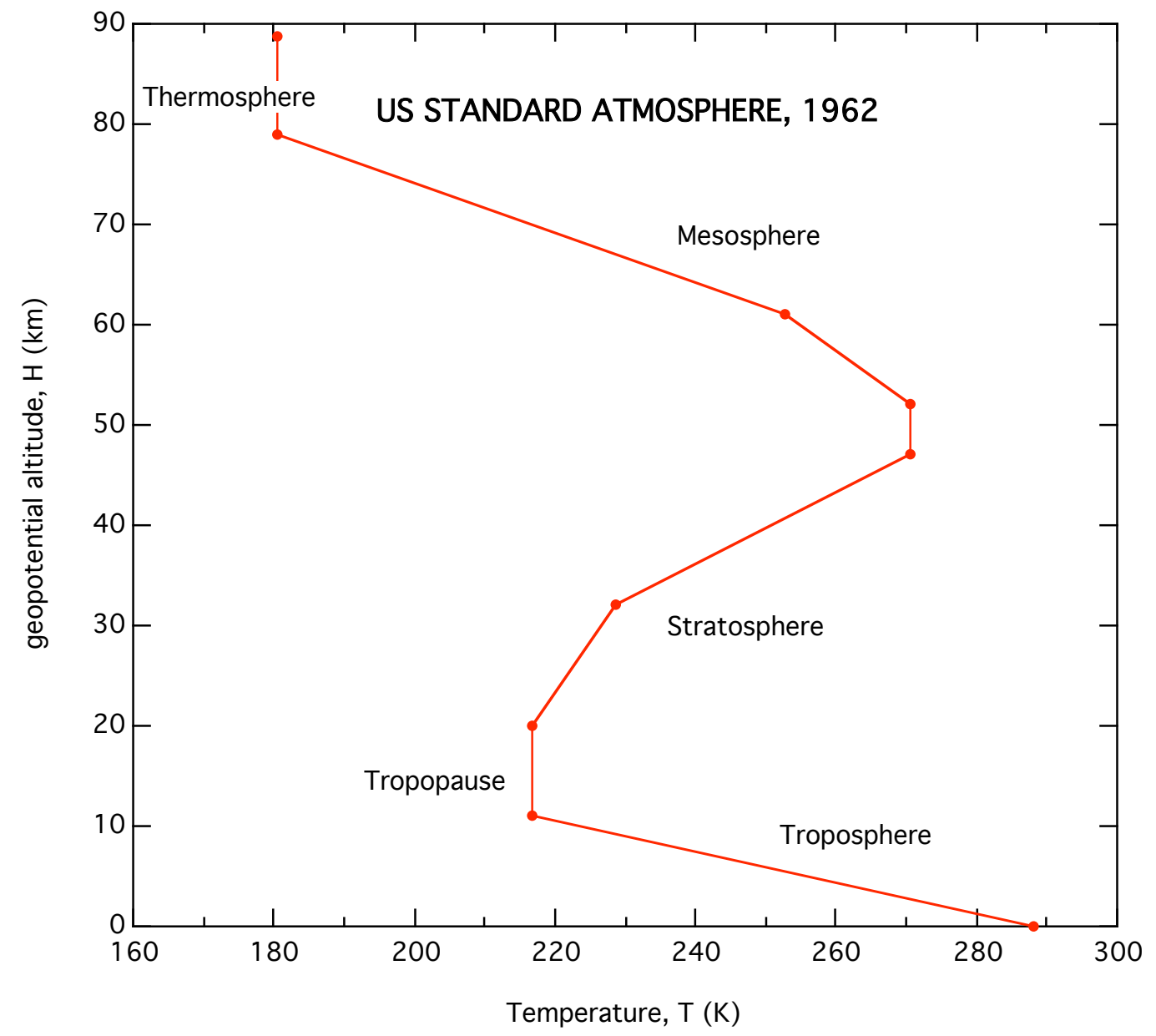

Figure B-1. Temperature profile of the US Standard Atmosphere 1962. 
Problem 1. Blast Waves from Nuclear Explosions

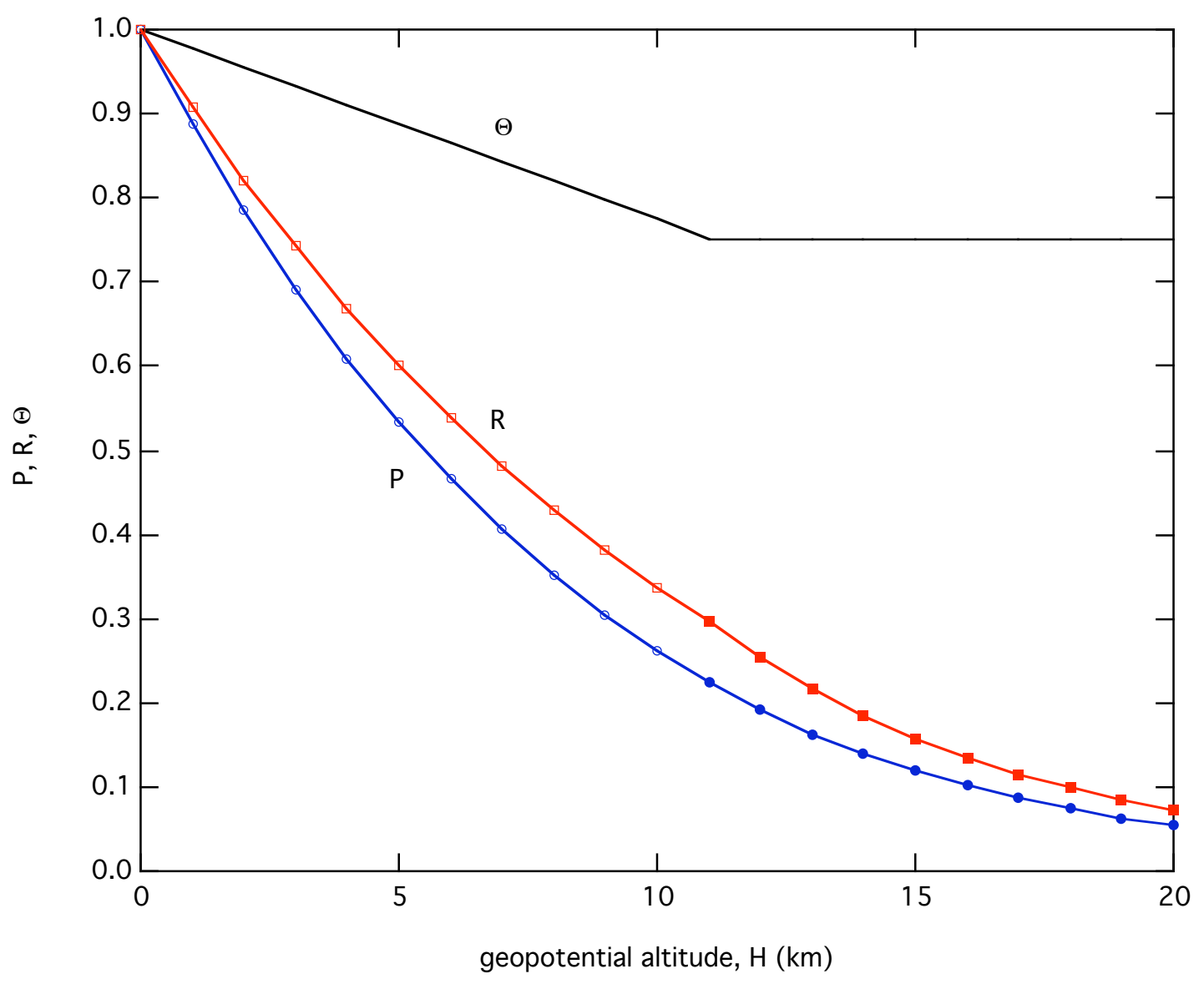

Figure B-2. Atmospheric structure based on the temperature profile of the US standard atmosphere 1962. 


\section{Problem 2: Blast Wave Reflections from Ideal Surfaces}

2.1 Regular Reflection from a Wedge $(M=2.05, \theta=60 \text { degrees, argon, } \gamma=1.3)^{7,8}$

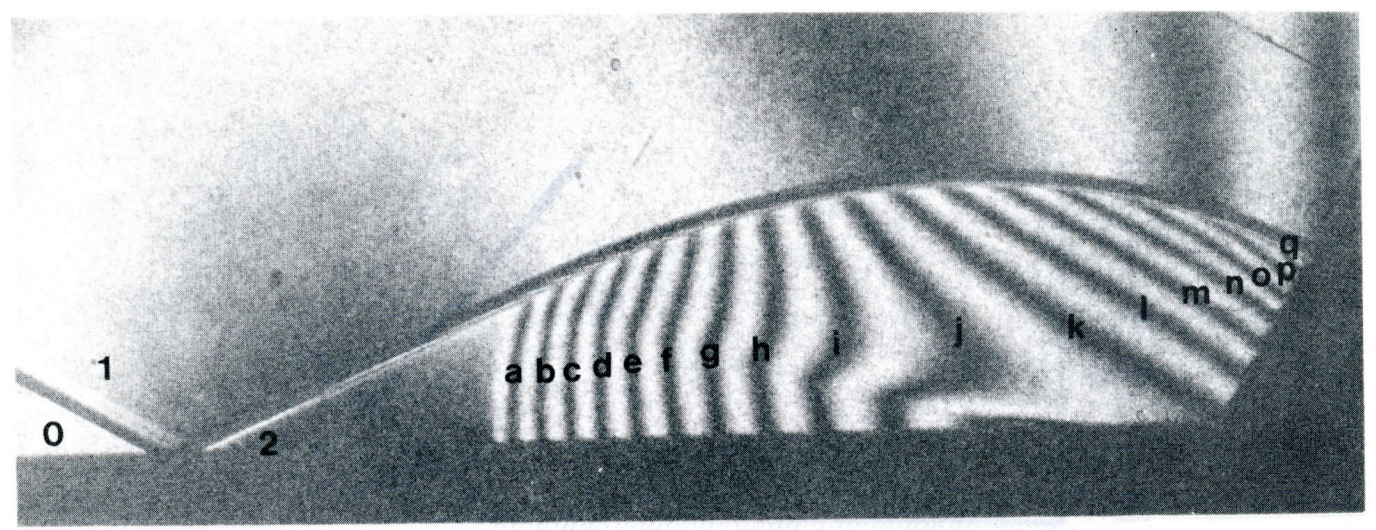

$\begin{array}{cccc}\text { Region } & \rho / \rho_{0} & \text { Region } & \rho / \rho_{0} \\ 0 & 1.00 & \mathrm{~h} & 3.86 \\ \mathrm{l} & 2.33 & \mathrm{i} & 3.80 \\ \mathrm{2} & 4.38 & \mathrm{j} & 3.73 \\ \mathrm{a} & 4.32 & \mathrm{k} & 3.67 \\ \mathrm{~b} & 4.25 & 1 & 3.60 \\ \mathrm{c} & 4.19 & \mathrm{~m} & 3.54 \\ \mathrm{~d} & 4.12 & \mathrm{n} & 3.47 \\ \mathrm{e} & 4.06 & \mathrm{o} & 3.41 \\ \mathrm{f} & 3.99 & \mathrm{p} & 3.34 \\ \mathrm{~g} & 3.93 & \mathrm{q} & 3.28\end{array}$

Figure 4 a - Interferogram

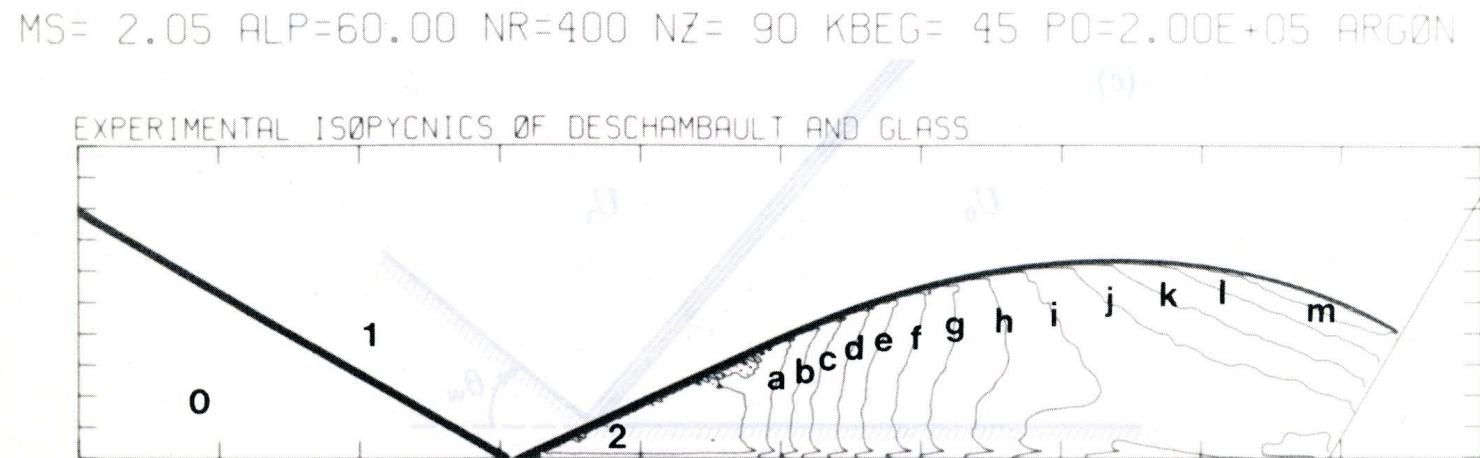

\footnotetext{
${ }^{7}$ H. M. Glaz, P. Colella, I. I. Glass, and R. L. Deschambault, A Detailed Numerical, Graphical and Experimental Study of Shock Wave Reflections, LBL-20033, Lawrence Berkeley Laboratory, 1985, 380 pp.

${ }^{8}$ H. M. Glaz, P. Colella, I. I. Glass, R. L. Deschambault (1985a) A numerical study of oblique shock-wave reflections with experimental comparisons. Proc. R. Soc. London, Ser. A, 398, pp. 117-140.
} 
2.2 Mach Reflection from a Wedge $(M=2.03, \theta=27 \text { degrees, air, } \gamma=1.4)^{7}$

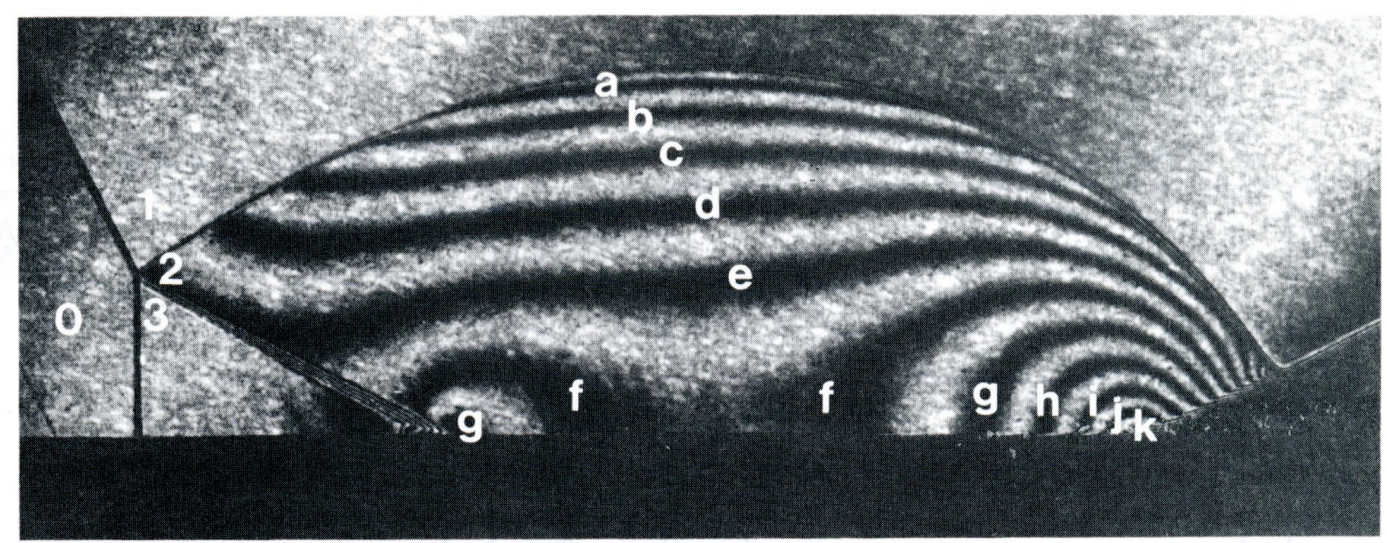

$\begin{array}{cc}\text { Region } & \rho / \rho_{0} \\ \text { l } & 1.00 \\ 2 & 3.68 \\ 3 & 3.33 \\ \text { a } & 3.36 \\ \text { b } & 3.44 \\ \text { c } & 3.52 \\ \text { d } & 3.60 \\ \text { e } & 3.68 \\ \text { f } & 3.75 \\ \text { g } & 3.83 \\ \text { h } & 3.91 \\ \text { i } & 3.99 \\ \text { j } & 4.06 \\ \text { k } & 4.14\end{array}$

Figure $15 a$ - Interferogram

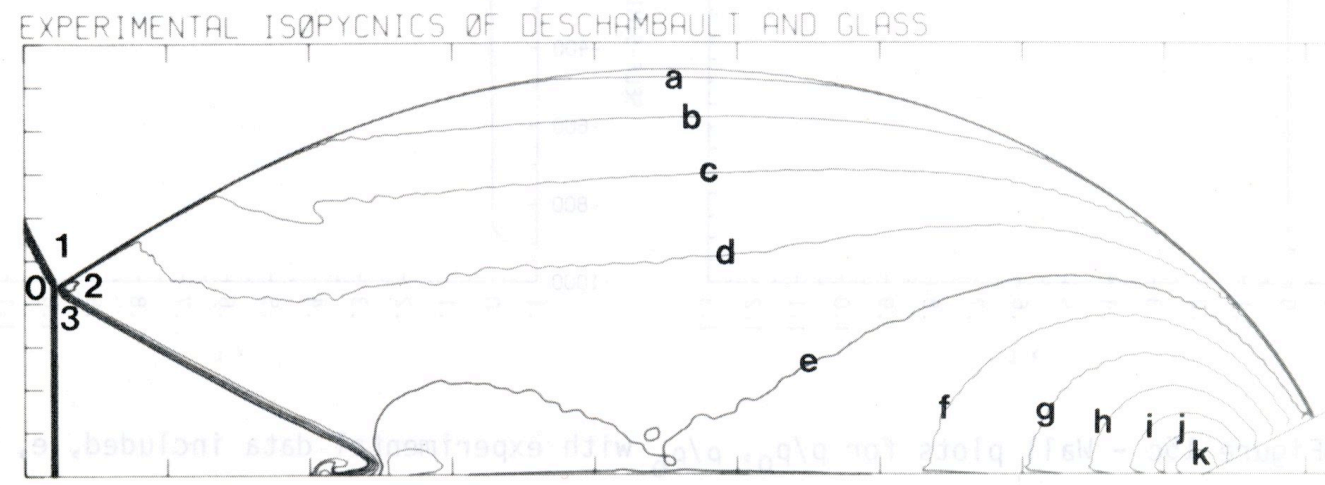


Problem 2. Blast Wave Reflections from Ideal Surfaces

2.3 Double Mach Reflection from a Wedge $(M=8.70, \theta=27 \text { degrees, air })^{7}$

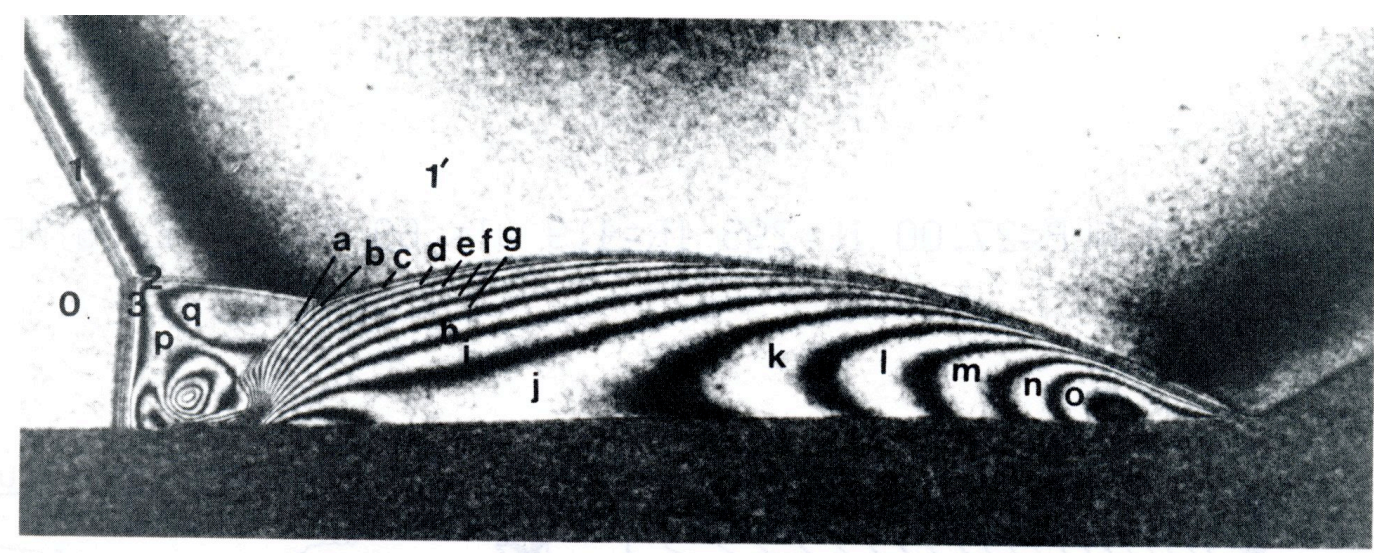

$\begin{array}{crcc}\text { Region } & \rho / \rho_{0} & \text { Region } & \rho / \rho_{0} \\ 0 & 1.00 & \mathrm{~g} & 13.32 \\ \mathrm{l} & 5.63 & \mathrm{~h} & 13.95 \\ \mathrm{I}^{\prime} & 6.89 & \mathrm{i} & 14.58 \\ 2 & 7.44 & \mathrm{j} & 15.21 \\ 3 & 5.74 & \mathrm{k} & 15.84 \\ \mathrm{a} & 9.53 & \mathrm{l} & 16.47 \\ \mathrm{~b} & 10.16 & \mathrm{~m} & 17.10 \\ \mathrm{c} & 10.79 & \mathrm{n} & 17.73 \\ \mathrm{~d} & 11.42 & \mathrm{o} & 18.36 \\ \mathrm{e} & 12.05 & \mathrm{p} & 6.37 \\ \mathrm{f} & 12.68 & \mathrm{q} & 8.07\end{array}$

Figure 16a - Interferogram

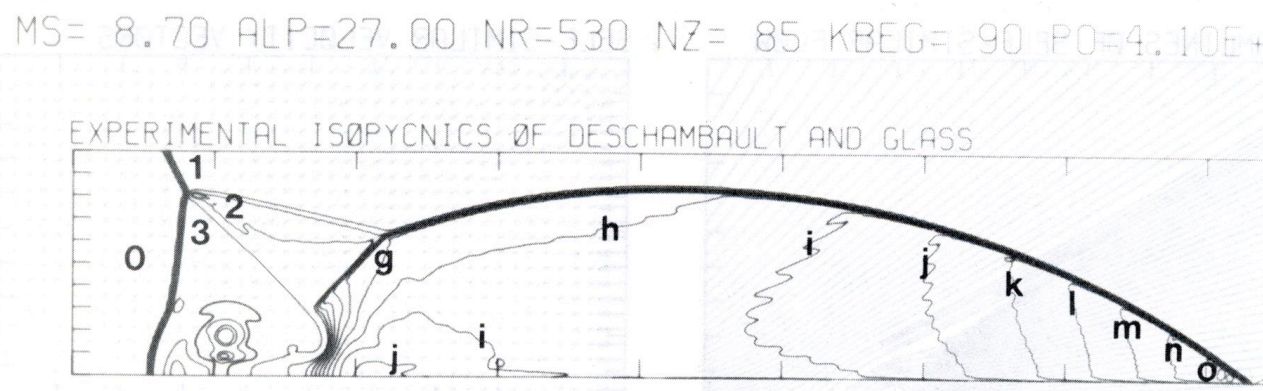


Problem 2. Blast Wave Reflections from Ideal Surfaces

Test Matrix: Shock Reflections from Wedges ${ }^{7}$

\begin{tabular}{|c|c|c|c|c|c|c|c|c|c|c|}
\hline CASE & FIGURE & GAS & TYPE & $\theta w$ & Ms & Po (k Pa) & po (gm/cc) & EOS & NX & NY \\
\hline 1 & 4 & Argon & RR & $60^{\circ}$ & 2.05 & 20.00 & $3.23 \times 10^{-4}$ & $\gamma=5 / 3$ & 355 & 90 \\
\hline 2 & 5 & Air & $\mathbf{R R}$ & $45^{\circ}$ & 1.26 & 101.12 & $1.146 \times 10^{-3}$ & $\begin{array}{l}\gamma=1.4 \\
\text { Hansen }\end{array}$ & 350 & 160 \\
\hline 3 & 6 & Air & SMR & $45^{\circ}$ & 1.50 & 50.66 & $5.73 \times 10^{-4}$ & $\begin{array}{l}\gamma=1.4 \\
\text { Hansen }\end{array}$ & 375 & 160 \\
\hline 4 & 7 & Air & DMR & $47^{\circ}$ & 3.03 & 3.33 & $3.77 \times 10^{-5}$ & $\begin{array}{l}\gamma=1.4 \\
\text { Hansen }\end{array}$ & 500 & 120 \\
\hline 5 & 8 & Air & CMR & $30^{\circ}$ & 2.65 & 13.33 & $1.52 \times 10^{-4}$ & $\begin{array}{l}\gamma=1.4 \\
\text { Hansen }\end{array}$ & 390 & 125 \\
\hline 6 & 9 & Argon & CMR & $30^{\circ}$ & 5.07 & 4.00 & $6.45 \times 10^{-5}$ & $\gamma=5 / 3$ & 420 & 140 \\
\hline 7 & 10 & Air & CMR & $10^{\circ}$ & 10.37 & 6.67 & $7.53 \times 10^{-5}$ & Hansen & 400 & 140 \\
\hline 8 & 11 & Air & SMR & $40^{\circ}$ & 1.66 & 33.33 & $3.8 \times 10^{-4}$ & $\begin{array}{l}\gamma=1.4 \\
\text { Hansen }\end{array}$ & 375 & 135 \\
\hline 9 & 12 & Air & DMR & $40^{\circ}$ & 2.87 & 16.67 & $1.9 \times 10^{-4}$ & $\begin{array}{l}\gamma=1.4 \\
\text { Hansen }\end{array}$ & 420 & 110 \\
\hline 10 & 13 & Air & DMR & $40^{\circ}$ & 3.72 & 6.00 & $6.87 \times 10^{-5}$ & $\begin{array}{l}\gamma=1.4 \\
\text { Hansen }\end{array}$ & 420 & 100 \\
\hline 11 & 14 & Air & DMR & $40^{\circ}$ & 4.62 & 2.80 & $3.19 \times 10^{-5}$ & $\begin{array}{l}\gamma=1.4 \\
\text { Hansen }\end{array}$ & 420 & 90 \\
\hline 12 & 15 & Air & SMR & $27^{\circ}$ & 2.03 & 33.33 & $3.87 \times 10^{-4}$ & $\gamma=1.4$ & 350 & 130 \\
\hline 13 & 16 & Air & DMR & $27^{\circ}$ & 8.70 & 4.10 & $4.76 \times 10^{-5}$ & Hansen & 440 & 85 \\
\hline 14 & 17 & Air & C/DMR & $20^{\circ}$ & 7.19 & 8.00 & $9.29 \times 10^{-5}$ & Hansen & 420 & 120 \\
\hline 15 & 18 & Air & DMR & $20^{\circ}$ & 8.86 & 4.10 & $4.65 \times 10^{4}$ & Hansen & 500 & 110 \\
\hline
\end{tabular}

\subsection{Shock Reflection Factors for Air}

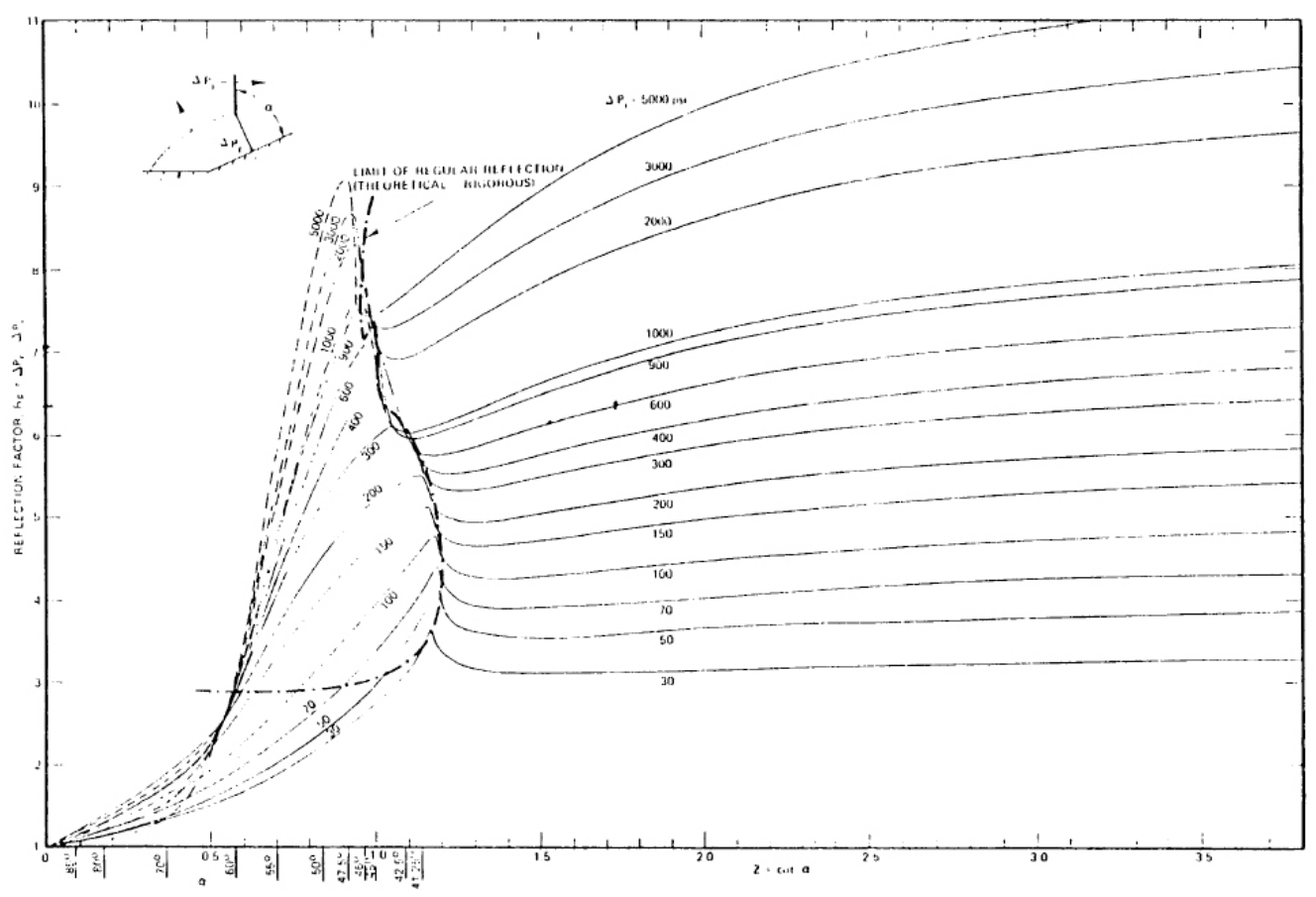

ADIABATIC AIR SHOCK REFLECTION FACTORS 


\title{
Problem 2. Blast Wave Reflections from Ideal Surfaces
}

\subsection{HE-Driven Blast Wave Reflection from a Surface ${ }^{9}$}

\begin{abstract}
Flow fields associated with one-dimensional free-air explosions are well known both for the point-source case and for the case of a blast wave driven by the detonation of a highexplosive (HE) charge. Considered here is the two-dimensional case of the reflection of a spherical, HE-driven blast wave from an ideal plane surface. The evolution of the flow field was calculated with a non-diffusive numerical algorithm for accurately solving the Euler equations. This algorithm is based on a second-order Godunov scheme and a monotonicity algorithm that is designed to give sharp shocks and contact surfaces while smooth regions of the flow remain smooth yet free of numerical diffusion. The incident HE-driven blast wave was accurately captured by a fine-zoned one-dimensional calculation that was continuously fed into the two-dimensional mesh. The latter incorporated a fine-zoned mesh that followed the reflection region and accurately resolved the complicated flow structure occurring on multiple length scales. Major findings in the regular reflection region were as follows. Portions of the main reflected shock reflected within the channel formed by the wall and the dense HE products, thus creating additional pressure pulses on the wall. Coherent vortex structures formed on the fireball as a result of the interaction of the reflected shock with this contact surface. The flow did indeed make a transition to a double-Mach structure, but this transition was delayed 1.5 to 3.8 degrees beyond the two-shock limit of regular reflection because the nascent Mach stem was less than one cell high in this region. The double-Mach structure with its two moving stagnation points was similar (but not identical) to an equivalent shock-on-wedge case. A key feature of this flow was a supersonic wall jet (velocity of 3.5 to $4.3 \mathrm{~km} / \mathrm{s}$ ) consisting of a free shear layer and a wall boundary layer. The wall jet was laminar in these calculations, but should actually be turbulent due to Reynolds number considerations. Nevertheless, calculated peak pressures were found to be in excellent agreement with experimental data at all ground ranges.
\end{abstract}

\section{Introduction}

Considered here is the two-dimensional axisymmetric reflection of a spherical highexplosives (HE)-driven blast wave from a plane surface. The temporal evolution of the flow field was calculated with a second-order Eulerian Godunov scheme that accurately solves such inviscid compressible flow problems on a very fine computational mesh. The accuracy of the solution was confirmed by experimental pressure data for the same problem.

The details of flow fields associated with one-dimensional free-air explosions are well established. Consider, for example, the similarity solutions for spherical blast waves: the point explosion solution of Taylor (1941) and Sedov (1946), and all classes of blast waves bounded by strong shocks (Oppenheim et ale 1972a) and by strong Chapman-Jouguet detonations (Oppenheim et ale 1972b). Other examples are the non-self-similar solutions of the decay of a point-source explosion: the original finite difference calculation (Von Neumann and Goldstine

${ }^{9}$ P. Colella, R. E. Ferguson, H. M. Glaz, A. L. Kuhl, Mach reflection from an HE-driven blast wave, Dynamics of Explosions, AIAA Progress in Astronautics and Aeronautics, vol. 106, 1985, pp. 388-421. 


\section{Problem 2. Blast Wave Reflections from Ideal Surfaces}

1955), the method of integral relations solution (Korobeinikov and Chushkin 1966), the method of characteristics solution (Okhotsimskii et ale 1957), and the Lagrangian finite difference calculations (Brode 1955). Also well established are non-self-similar solutions of the decay of spherical blast waves driven by a solid, high-explosives (HE) charge (Brode 1959).

However, when one considers the reflection of such spherical blast waves from a plane surface, a detailed description of the flow fields is not generally available. Such flows are inherently two-dimensional. They are driven by decaying blast waves, and hence they are intrinsically non-self-similar. They depend parametrically on the scaled height of burst (HOB) of the explosion, the blast source, and the equation of state (EOS) of the medium (e.g., y varies for real air). Hence, such flow fields are not amenable to general solution; each represents a particular case.

Much of our knowledge of such reflections comes from considering the flow field in the near vicinity of the reflection point. By neglecting the rarefaction wave behind the incident shock, one can equate the flow to that produced by a plane, square wave shock reflecting from a plane surface. This is, of course, a good approximation when the flow behind the reflected shock is supersonic (relative to the reflection point). Many tools then become available. For example, one can use the shock polar technique (Courant and Friedrichs 1948) with an appropriate equation of state to predict peak pressures in the regular reflection regime; whereas in the Mach reflection regime, one must resort to experimental data of shock reflections from wedges (e.g., Bertrand 1972). One can use experimental shock-on-wedge results and their associated empirical theories to predict the transition to Mach reflection and the approximate shock structure. ${ }^{10}$ Indeed, such analysis predicts that for strong shocks, transition will proceed from regular to double-Mach reflection. One can even view the height-of-burst problem as a continuous sequence of shock-onwedge configurations for which the wedge angle varies from 90 degrees at ground zero to 0 degrees at an infinite ground range. Nevertheless, such techniques have a limited utility. They are always approximations to a truly non-self-similar problem, and they do not describe the entire flowfield. To overcome such limitations, one must resort to height-of-burst experiments and two-dimensional numerical simulations.

Height-of-burst experiments utilizing HE blast wave sources have been conducted (Baker 1973). Typically, flow field measurements are limited to near-surface static and total pressure histories at a small number of ground ranges, and high-speed photography. Often there is much scatter in the data due to non-repeatability of the HE charges; this scatter limits the scientific usefulness of the data. Some of the most repeatable data come from tests performed with 8-1b spheres of PBX-9404 (Carpenter 1974). Nevertheless, such measurements are not sufficient to allow one to reconstruct the entire flow field. For that, a numerical simulation of the flow is required.

Today, one can simulate the reflection of a spherical blast wave from a plane surface with numerical codes that solve the inviscid two-dimensional Euler equations of gasdynamics: for example, a simulation of the Tunguska meteorite explosion at an $\mathrm{HOB}=305 \mathrm{~m} / \mathrm{KT}^{1 / 3}$

\footnotetext{
${ }^{10}$ See , for example, Ben-Dar and Glass (1978, 1979), Ando and Glass (1981), Shirouzu and Glass (1982), Lee and Glass (1984), Deschambault and Glass (1983), Bazhenova et al. (1984), Hu and Glass (1986), Hornung (1985), and Hornung and Taylor (1982).
} 


\section{Problem 2. Blast Wave Reflections from Ideal Surfaces}

(Shurshalov 1978) and the calculation of a point-source case detonated at an HOB $=31.7$ $\mathrm{m} / \mathrm{KT}^{1 / 3}$ (Fry et al., 1981). How accurate are such calculations? One of the difficulties in numerical simulation of such flows is the disparity of length scales in the problem; for example, the height-of-burst scale versus the Mach stem height (typically less than 1/10 the height-of-burst scale) versus the boundary layer scale (which is much smaller than the Mach stem height). One must take special care to design the computational mesh to take such disparate length scales into account. With the memory size and speed of class VI computers such as the CRAY I, such largescale computations are now possible (although expensive). Of course, one needs a minimaldiffusion numerical algorithm to maximize the information per grid point. A noteworthy example is the second-order Eulerian Godunov scheme of Colella and Glaz (1984, 1985). This code has been used to simulate shock-on-wedge experiments in the regular reflection regime and in the simple, complex, and double-Mach reflection regimes. Excellent agreement with data was obtained for those cases for which viscous and non-equilibrium effects were negligible in the experiments (Glaz et al. 1985a, 1985b, 1986). In some of the double-Mach reflection cases for which such effects were not small, qualitative agreement was still found for flowfield features such as contact surface/second Mach stem interaction and subsequent vortex rollup. Nevertheless, the question remains: How accurately can one numerically simulate the truly non-steady heightof-burst case?

The objective of this work was then to perform a highly resolved numerical simulation of the two-dimensional reflection of an HE-driven blast wave with the abovementioned Godunov scheme and to check the accuracy of the solution by comparing it with precision experimental data. An 8-1b PBX-9404 charge experiment detonated at $\mathrm{HOB}=51.66 \mathrm{~cm}$ (Carpenter 1974) was selected for that purpose. A zoning convergence study (with a fine grid mesh spacing of 1.2, 0.6, and, finally, $0.3 \mathrm{~mm}$ ) was performed to demonstrate that the results were independent of cell size.

The computational technique including the second-order Godunov scheme, the equations of state, the initial conditions, and the grid dynamics are described in $\$ 2$. The numerical results, such as the incident one-dimensional blast wave, the regular reflection regime, transition, the double-Mach reflection regime, comparisons of surface data, and comparisons with an equivalent shock-on-wedge case, are presented in $\S 3$. Conclusions and recommended improvements are offered in $\S 4$.

\section{Computational Method}

The equations of compressible hydrodynamics in one space variable, written in conservation form, are

where

$$
\frac{\partial}{\partial t} U+\frac{\partial}{\partial V} A F+\frac{\partial}{\partial r} H=0
$$




\section{Problem 2. Blast Wave Reflections from Ideal Surfaces}

$$
U=\left\{\begin{array}{l}
\rho \\
\rho u \\
\rho v \\
\rho E_{T} \\
\rho \chi
\end{array}\right\}, \quad F(U)=\left\{\begin{array}{l}
\rho u \\
\rho u^{2} \\
\rho u v \\
\rho u E_{T} \\
\rho u \chi
\end{array}\right\}, \quad H(U)=\left\{\begin{array}{l}
0 \\
p \\
0 \\
0 \\
0
\end{array}\right\}
$$

Here, $\rho$ is the density; $E_{T}=e+\left(u^{2}+v^{2}\right) / 2$ is the total energy per unit mass, where $e$ is the internal energy per unit mass, $u$ is the component of velocity in the r-direction, and $v$ is the transverse component of velocity; $p$ is the pressure; $\chi$ represents an arbitrary advected scalar quantity; and $V \equiv V(r)=r^{\alpha+1} /(\alpha+1)$ is a volume coordinate, $A \equiv A(r)=d V / d r=r^{\alpha}$. The values $\alpha=0,1,2$ correspond to Cartesian, cylindrical, and spherical symmetry, respectively. This particular representation of the equations follows Colella and Woodward (1984) and corresponds closely to the finite-difference equations that follow.

The pressure is given by an equation of state:

$$
p \equiv p(\rho, e)
$$

for single-fluid hydrodynamics. For the calculations presented here, it is necessary to use a twofluid model, where the two fluids are the detonation product gases and air. Each of these materials has associated with it an equation of state of the form of Eq. (2). We let $\chi$ denote the volume fraction of high explosives (HE), so that in a mixed cell, $0 \leq \chi \leq 1$. Then our two-fluid treatment is defined by the last equation in Eq. (1) and by setting

$$
p=\chi p_{H E}+(1-\chi) p_{\text {air }}
$$

wherever a pressure is needed by the numerical method. This relatively crude treatment (in particular, our reliance on the mixture density and internal energy precludes referring to the model as a true two-fluid model) turns out to be sufficient for the present problem. This is largely due to the fact that the dynamics of the material interface are not of major interest and they do not directly interact with the Mach stem region flow field, which is the focal point of this study. Our treatment here will be superseded by a true multi-material algorithm based on the simple line interface calculation (SLIC) algorithm of Noh and Woodward (1976) and the Eulerian second-order Godunov scheme for single-fluid hydrodynamics (Colella et al., 1986).

The numerical method used in this study is the version of the second-order Eulerian Godunov scheme described in Colella and Glaz (1985). This version was especially designed to handle general equations of state of the type encountered here. The modifications necessary for non-Cartesian symmetries (i.e., $\alpha=1$ or 2 ) are described in Colella and Woodward (1984). Operator splitting is used to solve multi-dimensional problems; in the axisymmetric calculation of $\S 3$, this means that Eq. (1) with $\alpha=1$ are solved in the radial direction with $u$ set to the radial component of velocity; and then Eq. (1) with $\alpha=0$ are solved in the axial direction with $u$ set to 


\section{Problem 2. Blast Wave Reflections from Ideal Surfaces}

the axial component of velocity. A brief overview of the method for solving Eq. (1) is presented below.

Let $U^{n}=\left\{U_{j}^{n}\right\}$ represent the cell-averaged solution at time level $t=t^{n}$, i.e.,

$$
U_{j}^{n}=\left(\Delta V_{j}\right)^{-1} \int_{V_{j-1 / 2}^{n}}^{V_{j+1 / 2}^{n}} U\left(r, t^{n}\right) d V
$$

The computational objective is to define $U^{n+1}$ in terms of $U^{n}$. The conservative, second-orderin-time, finite-difference representation of Eq. (1) is:

$$
\Delta V_{j}^{n+1} U_{j}^{n+1}=\Delta V_{j}^{n} U_{j}^{n}-\Delta t^{n}\left[A_{j+1 / 2}^{n+1 / 2} F_{j+1 / 2}^{n+1 / 2}-A_{j-1 / 2}^{n+1 / 2} F_{j-1 / 2}^{n+1 / 2}+\left(H_{j+1 / 2}^{n+1 / 2}-H_{j-1 / 2}^{n+1 / 2}\right) \frac{2}{\Delta r_{j}^{n}+\Delta r_{j}^{n+1}}\right]
$$

where

$$
\Delta V_{j}=V_{j+1 / 2}-V_{j-1 / 2}, V_{j+1 / 2} \equiv V\left(r_{j+1 / 2}\right) \text { and } A_{j+1 / 2}^{n+1 / 2}=\left(\Delta t^{n}\right)^{-1} \int_{0}^{1} A\left[r_{j+1 / 2}^{n}+\beta\left(r_{j+1 / 2}^{n+1}-r_{j+1 / 2}^{n}\right)\right] d \beta
$$

Here, typically $F_{j+1 / 2}^{n+1 / 2}=F\left(U_{j+1 / 2}^{n+1 / 2}\right)$, and $U_{j+1 / 2}^{n+1 / 2}$ represents the average of $U$ along the $(j, j+1)$ interface, i.e.,

$$
U_{j+1 / 2}^{n+1 / 2}=\left(\Delta t^{n}\right)^{-1} \int_{t^{n}}^{t^{n+1}} U\left[r_{j+1 / 2}^{n}+s_{j+1 / 2}^{n+1 / 2}\left(t-t^{n}\right), t\right] d t \quad \text { where } \quad s_{j+1 / 2}^{n+1 / 2}=\left(r_{j+1 / 2}^{n+1 / 2}-r_{j+1 / 2}^{n}\right) / \Delta t
$$

Evidently, a computational scheme in the form Eq. (5) is defined by specifying $U_{j+1 / 2}^{n+1 / 2}$ as a function of $U^{n}$.

The first order Godunov scheme is defined by setting $U_{j+1 / 2}^{n+1 / 2}$ to the solution of the Riemann problem $\left(U_{j}^{n}, U_{j+1}^{n}\right)$ evaluated along the line $r / t=s_{j+1 / 2}^{n+1 / 2}$. The high-order scheme is conceptually similar in that the Riemann problem $\left(U_{j+1 / 2, L}^{n}, U_{j+1 / 2, R}^{n}\right)$ is constructed and solved in the same way. However, the left and right states are now functions of $\left(U_{j-2}^{n}, \ldots U_{j+2}^{n}\right)$, respectively. These additional data are used to create monotonized piecewise-linear profiles in each computational zone, from which a version of the method of characteristics is based to get new values centered on the interface. The overall construction, including the solution of the Riemann problem, is equivalent to the method of characteristics (up to second order) for smooth flow in determining the interface fluxes. Further details, such as monotonicity constraints and additional constructions necessary near strong discontinuities, may be found in the references mentioned.

An important aspect of our numerical method is that we do not require equation-of-state evaluations at each step in the Riemann problem iterative solution; it is only necessary for the 


\section{Problem 2. Blast Wave Reflections from Ideal Surfaces}

approximate method that the equation of state be evaluated for each $U_{j}^{n}$. The information required by the algorithm are the dimensionless quantities $\gamma \equiv \gamma(\rho, e)$ and $\Gamma \equiv \Gamma(\rho, e)$ such that

and

$$
p=(\gamma-1) \rho e
$$

$$
c^{2}=\Gamma p / \rho
$$

where $c$ is the speed of sound. Note that $\gamma \neq \Gamma$ for a non-polytropic equation of state.

The equations of state used in the calculations of $\S 3$ are the equilibrium air EOS of Gilmore (1955) and Hansen (1959), and the Jones-Wilkins-Lee (JWL) EOS for PBX-9404 detonation product gases (Dobratz 1974). The caloric JWL equation of state takes the form

$$
p=A\left(1-\omega \rho_{0} / R_{1} \rho\right) e^{-R_{1} \rho_{0} / \rho}+B\left(1-\omega \rho_{0} / R_{2} \rho\right) e^{-R_{2} \rho_{0} / \rho}+\omega \rho e
$$

whereas the isentrope is given by

$$
p=A e^{-R_{1} \rho_{0} / \rho}+B e^{-R_{2} \rho_{0} / \rho}+C\left(\rho_{0} / \rho\right)^{-(\omega+1)}
$$

where $\rho_{0}$ is the initial charge density and the JWL parameters for PBX-9404 are $\mathrm{A}=8.545$ Mbar; $\mathrm{B}=0.2049$ Mbar; $\mathrm{C}=0.00754 \mathrm{Mbar} ; \mathrm{R}_{1}=4.60 ; \mathrm{R}_{2}=1.35 ; \omega=0.25$. The behavior of $\gamma$ for the JWL EOS may be found by fitting Eq. (6) to Eq. (8), and $\Gamma(\rho, e)$ can be calculated from the isentrope using Eq. (9); in this case. $c^{2}$ is obtained in closed form (Glaz, 1979) and Eq. (7) may be used to calculate $\Gamma$.

The calculation was run in two stages: first as a one-dimensional free air burst until ground strike, and then as a two-dimensional reflection problem. The one-dimensional calculation was initialized when the detonation wave reached the charge radius $R_{c}$. The flow field inside the charge at that time was assumed to be that of an ideal Chapman-Jouguet (CJ) detonation (Taylor 1950; Kuhl and Seizew 1978) with no afterburning. Using the JWL parameters for a PBX-9404 charge with an initial density of $\rho_{0}=1.84 \mathrm{~g} / \mathrm{cm}^{3}$, the CJ state is: $p_{C J}=370 \mathrm{kbars} ; \rho_{C J}=2.485 \mathrm{~g} / \mathrm{cm}^{3} ; e_{C J}=8.142 \times 10^{10} \mathrm{erg} / \mathrm{g} ; W_{C J}=8.8 \mathrm{~km} / \mathrm{s} ; u_{C J}=2.28 \mathrm{~km} / \mathrm{s}$ $q_{C J}=5.543 \times 10^{10} \mathrm{erg} / \mathrm{g} ; \Gamma=2.85 ; \chi=1$.

For an $8-1 \mathrm{~b}$ sphere the charge radius was $R_{c}=7.76 \mathrm{~cm}$. The ambient atmosphere was initialized as $p_{a}=1.00$ bar; $\rho_{a}=1.1687 \times 10-3 \mathrm{~g} / \mathrm{cm}^{3} ; e_{a}=2.1390 \times 10^{9} \mathrm{erg} / \mathrm{g} ; u=0 ; \chi=0$. A fine-zoned grid $(\Delta r=0.3 \mathrm{~mm}$ ) was dynamically moved with the shock to accurately capture the complex flow in that region. Coarse zones $(\Delta r=3 \mathrm{~mm})$ were used near $r=0$ and for large $r$; and a transition region connected these cells with the fine grid. After initialization, the evolution of the one-dimensional blast wave was calculated by solving Eqs. (1) with $\alpha=2$ until the shock radius was equal to the height of burst $(51.66 \mathrm{~cm} ; t=97.44 \mu \mathrm{s})$. This solution was then conservatively interpolated onto a two-dimensional mesh. 


\section{Problem 2. Blast Wave Reflections from Ideal Surfaces}

The two-dimensional mesh covered a region $0 \leq r \leq 100 \mathrm{~cm}$ and $0 \leq z \leq 20 \mathrm{~cm}$ (617 r cells by $214 z$ cells). Note that the top of the grid was below the height of burst to pack as many cells near the wall as possible. During the computation, the reflected shock never reached the upper boundary; consequently, it could be treated with a time-dependent Dirichlet boundary condition. The Dirichlet data were provided by continuing to update the one-dimensional solution for each step of the two-dimensional calculation and feeding this solution into the top boundary. The bottom boundary was treated as an ideal (slip flow) reflecting plane. The left and right boundaries were treated as a symmetry line and an outflow boundary, respectively. A uniform fine-grid region ( $267 \mathrm{r}$ cells by $140 \mathrm{z}$ cells) with $\Delta r=\Delta z=0.3 \mathrm{~mm}$ was dynamically moved to follow the rightmost shock (the incident wave at early times and the Mach stem at late times). Again, transition and coarse cells $(\Delta r=\Delta z=3 \mathrm{~mm})$ were used around the fine-grid region. The two-dimensional calculation was continued until the Mach reflection point reached $80 \mathrm{~cm}$ $(270 \mu s)$. This required 3200 computational steps and about 9 hours CP time on the CRAY 1.

\section{Results}

\subsection{Incident HE-Driven Blast Wave}

In 1959, Brode performed a pioneering calculation of a spherically symmetric blast wave driven by the detonation of a spherical TNT charge (initial charge density of $1.5 \mathrm{~g} / \mathrm{cm}^{3}$, detonation pressure of $157 \mathrm{kbar}$ ). The one-dimensional Lagrangian finite-difference scheme used the artificial viscosity technique (Von Neumann and Richtmeyer, 1950) to capture shock fronts, and variable gamma equations of state to describe the air and detonation products gases. He found that an extremely strong rarefaction wave was created when the detonation wave reached the radius of the charge. This rarefaction accelerated the detonation products to a velocity of about $5.5 \mathrm{~km} / \mathrm{s}$. The interface or contact surface, CS, between the air and the detonation products acted like a spherical piston - thus creating an air shock (maximum peak pressure of about 400 bars). The resulting blast wave behaved like a decaying piston-driven blast wave (Sedov 1959) for shock pressures greater than about 7 bars, and approached the point-source similarity solution thereafter. The aforementioned rarefaction wave caused the detonation products to over-expand to a velocity larger than that induced by the air shock. This incompatibility was resolved by an inward-facing shock, which eventually imploded and created a series of secondary pulses at late times.

Our calculation was performed for a spherical PBX-9404 charge (initial charge density of $1.84 \mathrm{~g} / \mathrm{cm}^{3}$ and detonation pressure of $370 \mathrm{kbar}$ ). The resulting blast wave was qualitatively similar to Brode's results; hence, the results will not be reported here in detail. Quantitative differences were as follows. Peak velocities reached about $17 \mathrm{~km} / \mathrm{s}$, whereas the maximum peak air-shock pressure reached about $1 \mathrm{kbar}$, owing to the larger detonation pressure of the PBX charge. The blast wave approached the point-source solution at a shock overpressure of about 13 bars versus 7 bars for TNT. The air shock arrived at ground zero (i.e., at a shock radius corresponding to the $\mathrm{HOB}=51.66 \mathrm{~cm}$, or 6.78 charge radii) with an incident over-pressure of 98.86 bars; hence, the flow field corresponded to a piston-driven wave throughout the entire regime of the two-dimensional calculation. This led to shock interactions that are unique to the HE case. 


\section{Problem 2. Blast Wave Reflections from Ideal Surfaces}

\subsection{Overall View of the Two-Dimensional Reflection}

An overall view of the two-dimensional reflection of the spherical HE-driven blast wave from an ideal plane surface is depicted in Fig. 1 in terms of isochoric, iso-energy, and isobaric contours at different times. Thirty equally spaced contour values were used, with the minimum and maximum value and step size identified on the plot. This technique gives a concise display of the major features of the two-dimensional flow field: discontinuities appear as heavy dark lines (where many contours group together), rarefaction waves appear as a fan of contour lines, while plateau regions are contour-free. Contact surfaces may be identified as discontinuities in density and internal energy, without any jump in pressure or velocity; slip lines may be distinguished as contact surfaces with a discontinuous change in velocity; shocks are denoted by discontinuities with sharp jumps in pressure.

In Fig. I, the incident shock (I), the contact surface (CS) separating the detonation products and air, and the inward-facing shock ( $\left.\mathrm{I}^{\prime}\right)$ of the incident blast wave are clearly visible. Reflection of the incident shock I from the plane surface creates the main reflected shock R, which effectively stops the contact surface CS. At small ground ranges, the reflected shock propagates upward very slowly because of the large, downward-directed dynamic pressure of the detonation products gases of the incident wave. This is markedly different from the case of the reflection of a point explosion blast wave in which the reflected shock propagates very rapidly through the low-density, high-sound-speed region near the blast center (Fry et a1. 1981).

Interactions of the reflected shock R with the contact surface CS and the shock I' create additional shocks near the ground and generate vortex structures on CS and SL', as shown in Figs. la and $\mathrm{lb}$. The reflected shock also deflects the contact surface away from the Mach stem region so that in this calculation, the detonation products are not entrained in the Mach stem flow.

\subsection{The Regular Reflection Region}

A detailed view of the flow field near the end of the regular reflection region $(t=171 \mu s$, reflection point at $50 \mathrm{~cm}$ ) is shown in Figs. $2 \mathrm{a}$ and $2 \mathrm{~b}$. The weaker discontinuities are somewhat difficult to pick out when they are located in the coarse-zoned region; hence they have been depicted schematically in Fig. 2c.

The reflected shock $\mathrm{R}$ interacts with the incident-wave contact surface $\mathrm{CS}$ at point $\mathrm{A}$, creating a reflected shock $\mathrm{RC} 1$ and deflecting $\mathrm{CS}$. Shock $\mathrm{RC} 1$ reflects off the wall at point $\mathrm{B}$ as a regular reflection, thus creating a second peak pressure on the wall. The reflected portion of $\mathrm{RC} 1$ reflects off contact surface $\mathrm{CS}$ at point $\mathrm{C}$, creating reflected shock RC2, and further deflects contact surface CS. Shock RC2 reflects off the wall at point D as a regular reflection, thus creating a third peak pressure on the wall. The reflected portion of shock RC2 reflects off contact surface $\mathrm{CS}$ at point E, creating a third reflected shock RC3.

The transmitted portion of shock $\mathrm{R}$ emanating from point $\mathrm{A}$ interacts with the incident shock I' (oblique shock interaction) at point F, creating a slip line SL'. The transmitted portion of shock I' emanating from point $\mathrm{F}$ interacts obliquely with the transmitted portion of shock RC1 emanating from $\mathrm{C}$ at point $\mathrm{G}$; transmitted shocks from this reflection interact with the contact 


\section{Problem 2. Blast Wave Reflections from Ideal Surfaces}

surface CS at point H, and with slip line SL' at J. At earlier times, a transmitted shock from point $\mathrm{J}$ interacted with the main reflected shock at point L. Density/internal energy gradients in the incident wave cause kinks in the main reflected wave at points $\mathrm{K}$ and $\mathrm{K}$ '.

In summary, the following features were found in the regular reflection region. The main reflected wave $\mathrm{R}$ reflects within the channel formed by the wall and the dense detonation products (CS), causing additional pressure pulses on the wall. Shock interactions with contact surfaces at points $\mathrm{A}$ and $\mathrm{F}$ inviscidly generate positive and negative vorticity, respectively, which rolls up into vortex structures shown in Figs. la and lb. Finally, the main contact surface CS is idealized in this calculation as a discontinuity. We know experimentally, however, that this surface is irregular and diffused - perturbations on this surface grow owing to a Rayleigh-Taylor mechanism and these lead to local turbulent mixing during the evolution of the incident blast wave (Anisimov et al. 1983). The strength of reflected shocks RC1 and RC2 will depend on the mixing across the contact surface CS. These inviscid calculations, which do not take into account such turbulent mixing, no doubt overestimate the strength of shocks RC1 and RC2.

Table 1 Comparison of regular to double-Mach transitions

\begin{tabular}{|l|c|c|c|}
\hline Source & $\begin{array}{c}\text { Wedge angle } \\
\theta \text { (degrees) }\end{array}$ & $\begin{array}{c}\text { Incident shock angle } \\
\alpha \text { (degrees) }\end{array}$ & $\begin{array}{c}\text { Transition } \\
\text { ground range (cm) }\end{array}$ \\
\hline $\begin{array}{l}\text { Limit of regular reflection: } \\
\text { Kuhl (1982) }\end{array}$ & 47 & 43 & 48.2 \\
Gilmore EOS for air & 46 & 44 & 49.9 \\
Glass (1982) & & & \\
Hansen EOS for air & & & \\
HOB calculation: & 45.5 & 44.5 & 52.5 \\
PRR & 43.2 & 46.8 & 55 \\
DMR & & & \\
\hline
\end{tabular}

\subsection{The Transition Regime}

A detailed view of the shock structure in the transition region is given in Fig. 3. In this calculation, transition from regular reflection (RR) to double-Mach reflection (DMR) occurred at a ground range of greater than $52.5 \mathrm{~cm}$ and less than $55 \mathrm{~cm}$, with corresponding incident shock angles of 44.5 and 46.8 degrees, respectively. Comparison with the limit of existence regular reflection (i.e., the so-called deflection criterion) for real air in Table 1 indicates that the calculated regular reflection region persisted in this height-of-burst calculation for 1.5 to 3.8 degrees beyond the theoretical limit. Note that a similar persistence of regular reflection (PRR) has been observed for shock reflections from wedges, both experimentally (Bleackney and Taub 1949; Henderson and Lozzi 1975) and numerically, with the same hydro code used here (Glaz et a1. 1985a, 1985b, 1986).

One can identify three potential reasons for persistence of regular reflection: 1) real viscosity, 2) numerical viscosity, and 3) inadequate zoning. Real viscosity must be rejected for this case because it was not included in the calculation. The second-order Godunov scheme used here leaves essentially no numerical viscosity in the smooth regions of the flow. However, all shock-capturing schemes introduce numerical dissipation at shock fronts to allow a smooth 


\section{Problem 2. Blast Wave Reflections from Ideal Surfaces}

transition between pre-shock and post-shock states. When such algorithms are used to calculate shock waves near a wall, a "numerical wall boundary layer" is formed (Noh 1976). The primary effect of this is to create an artificial "wall heating"-typically a few percent. This effect can be seen in the density and radial velocity contours of Fig. 2, which exhibit a kink at about the 3-mm height (about ten cells).

A concerted effort was made to minimize computational cell-size effects. The 617 by 214 grid used essentially all of the one mega-word fast core space available on a CRAY 1 computer. The fine-zoned grid ( 267 by 140 cells) that slid with the reflection region used cells of 0.3 by 0.3 $\mathrm{mm}$. This resulted in 83 radial cells between the reflection point at $52.5 \mathrm{~cm}$ and the $55-\mathrm{cm}$ point, and one would think that would constitute adequate zoning. However, the Mach stem grows from a point (in the inviscid theory) and is never captured computationally until the shock structure grows large enough to be resolved on the mesh. Note that at the $55-\mathrm{cm}$ location, the Mach stem was only four cells high. If a Mach stem existed at the $52.5-\mathrm{cm}$ ground range, it would be less than one cell high; hence, it would not have been resolved. A more detailed inviscid calculation of transition using a local adaptive grid refinement (e.g., Berger and Colella 1986) is required to conclusively resolve this zoning question. We speculate that such inviscid calculations will indeed confirm that double-Mach reflection will exist immediately after passing the RR limit. Therefore, we believe that the persistence of regular reflection in these calculations is caused by inadequate zoning and the numerical wall boundary layer, while the persistence behavior observed in experiments is due to a viscous wall boundary effect. To conclusively prove the latter, a viscous calculation of the oblique shock structure at the wall is required.

Peculiar gasdynamic effects were observed in this calculation in the PRR region. It is well known that as the incident shock angle increases in the regular reflection region, one encounters the sonic criterion (where sound waves can reach all the way to the reflection point) about 1 degree before one reaches the RR limit (Henderson and Lozzi 1975). In such a case, the reflected shock is no longer straight but continuously curved near the reflection point. The present calculations also exhibit such effects. Figure 3 shows that the reflected shock is straight at a ground range of $45 \mathrm{~cm}(\mathrm{RR})$ but curved near the reflection point at $52.5 \mathrm{~cm}$. As shown in Table 2, the angle of the main portion of the reflected shock increases smoothly through transition; however, at the wall it jumps from about 24 degrees at the $50-\mathrm{cm}$ range to about 37 degrees at the $52.5-\mathrm{cm}$ range.

The pressure and velocity profiles on the wall also changed dramatically in the PRR region. When the reflection point was at $45 \mathrm{~cm}$, the pressure and velocity gradients were well behaved. However, in the PRR region (e.g., with the reflection point at $52.5 \mathrm{~cm}$ ), the pressure and velocity gradients on the wall become very large as one approaches the reflection point from the left.

In summary, the limit of regular reflection for this case is 43 to 44 degrees (depending on the particular equation of state used for air) with a corresponding ground range to transition of 48.2 to $49.9 \mathrm{~cm}$. In this calculation, regular reflection seemed to persist to a ground range of about $52.5 \mathrm{~cm}(\alpha=44.5$ degrees), but the reflected shock angle near the surface at this range was consistent with that of double-Mach reflection ( $\beta \sim 37$ degrees). Hence, we believe that it was indeed a nascent double-Mach structure that was not computationally resolved on the mesh. 


\section{Problem 2. Blast Wave Reflections from Ideal Surfaces}

As we shall see in the next section, both local adaptive mesh refinement and turbulence modeling are required to properly model certain details of the flow in the double Mach region and, by implication, to accurately predict transition.

Table 2 Shock angles near transition

\begin{tabular}{|c|c|c|c|c|}
\hline $\begin{array}{c}\text { Ground range } \\
(\mathrm{cm})\end{array}$ & $\begin{array}{c}\text { Incident shock } \\
\text { angle } \alpha(\mathrm{deg})\end{array}$ & $\begin{array}{c}\text { Off wall } \\
\beta(\mathrm{deg})\end{array}$ & $\begin{array}{c}\text { Reflected shock angle } \\
\text { Near wall } \\
\beta_{0}(\mathrm{deg})\end{array}$ & Regime \\
\hline 45 & 42.5 & 19 & 19 & RR \\
\hline 50 & 44 & 24 & 24 & PRR \\
\hline 52.5 & 46.5 & 26 & $\sim 37$ & PRR \\
\hline 55 & 48 & 28.5 & $\sim 37$ & DMR \\
\hline 57.5 & 49 & 33 & $\sim 37$ & DMR \\
\hline 60 & 51 & 34 & $\sim 37$ & DMR \\
\hline
\end{tabular}

\subsection{The Double-Mach Region}

A detailed view of the complex flow field in the double-Mach region is presented in Fig. $4(t=270 \mu \mathrm{s}$, Mach stem at $80 \mathrm{~cm})$. The domain of these figures represents the fine-zoned region of the calculation ( $267 \mathrm{r}$ cells by $140 \mathrm{z}$ cells with a cell size of $0.3 \mathrm{~mm}$ ). The incident shock (I), the reflected shock ( $\left.\mathrm{R}^{\prime}\right)$, and the main Mach stem (Ml) meet at the main triple point (TP1), generating a slip line (SL1) that has positive vorticity. Air flows along SL1 and impacts on the wall, thus creating a large local pressure. This point actually corresponds to a moving stagnation point (SP1), which is particularly evident in the relative vector velocity plot of Fig. 4c (the coordinate system is moving with the velocity of SP1 at $2.308 \mathrm{~km} / \mathrm{s}$ ). The flow over-expands from SP1 (about 145 bars) by means of a strong rarefaction wave (RW) and forms a lowpressure ( 10-bar minimum) supersonic wall jet (see the Mach number contours, relative to SP1, of Fig. 4c).

The gas velocity in the wall jet ( 3.5 to $4.3 \mathrm{~km} / \mathrm{s}$ ) is larger than the wave velocity of the Mach stem $(\sim 2.75 \mathrm{~km} / \mathrm{s})$, so the jet rams into the rear of the Mach stem. This interaction pushes out the foot of the Mach stem and forces the jet to expand upward two dimensionally, thus forming a rotational flow and a main vortex VI, which has positive rotation. The rotational flow near VI is locally supersonic, and embedded shocks ( $\mathrm{S}^{\prime}, \mathrm{S}^{\prime \prime}$. and $\left.\mathrm{S}^{\prime \prime \prime}\right)$ can be seen.

The toeing-out of the Mach stem creates a second triple point, TP2. This is actually an inverted Mach stem structure with an incident shock M1', a reflected shock S", a Mach stem M1, and a slip line SL2 that has negative vorticity. This slip line flows up and over the main vortex V1, approaches the wall and stagnates, thus creating a second moving stagnation point (SP2), which is also evident in the relative velocity vector plot of Fig. 4c. At a range of $80 \mathrm{~cm}, \mathrm{SP} 2$ has shocked-up on the wall. All of slip line SL2 and some of SL1 are entrained in a second vortex structure V2, which has negative rotation (see Fig. 4c). All of the fluid entering the main Mach stem M1 between triple points TP1 and TP2 is entrained in vortex V2 (see the vorticity contour plot of Fig. 4c). 


\section{Problem 2. Blast Wave Reflections from Ideal Surfaces}

The gas velocity is supersonic above slip line SL1 and subsonic below it. Pressure waves from SP1 coalesce, forming the second Mach stem M2. The latter interacts with the reflected shock forming a third triple point (TP3). This also appears to be an inverted Mach structure with an incident wave R', a reflected wave M2, a Mach stem R, and a slip line (SL3). A fourth triple point (TP4) can be seen on shock M2. It appears to be a remnant of the interaction of the embedded shock S"' with SL1. It has an incident shock M2, a reflected shock R", a Mach stem M2', and a slip line SL4. Shock R" terminates on slip line SL3, while shock M2' terminates on slip line SL1.

Secondary vortex structures are evident on slip line SL1 (caused by shock M2 and local rarefaction waves) and slip line SL2 (induced by shock S"'), and near vortex V1 (the entrained part of SL1 that was shocked by S").

The rarefaction wave behind the incident shock propagates through the DMR structure (see, for example, the density, internal energy, and pressure contour plots of Fig. 4a) just as in the regular reflection case; but this appears to be a weak effect, since the main discontinuities (R', SL1 and M2) are basically straight lines.

Even at the $80-\mathrm{cm}$ range, the wall jet was quite thin $(1.8 \mathrm{~mm})$ and not well resolved (about six cells high); although very fine zoning was used here, it was still too coarse for adequate numerical resolution. The slip line SL1 on top of the jet is a free shear layer subject to KelvinHelmholtz instabilities. Here the Reynolds number of the jet was about $3 \times 10^{4}$ based on jet height. It will no doubt develop vortex structures, leading to turbulent mixing. Also, the wall boundary layer will reduce the radial momentum of the jet. Hence, turbulence effects will influence the entrainment of the main vortex V1 and the toeing-out of M1' (i.e., there will be less pushing). These effects were not modeled in this calculation and may, in fact, influence transition.

\subsection{Surface Data}

Figure 5 gives a detailed snapshot of the complete flowfield on the surface at the end of regular reflection $(\mathrm{t}=171 \mu \mathrm{s}$, reflection point at $50 \mathrm{~cm})$ and in the fully resolved double-Mach region $(t=270 \mu \mathrm{s}$, Mach stem toe at $80 \mathrm{~cm})$. Such plots augment the interpretation of the contour plots in Figs. 2 and 4. The reflected shocks R, RC1, and RC2 (as well as additional wave structures near the origin) can be seen. Both $\mathrm{RC} 1$ and $\mathrm{RC} 2$ reside in the coarse-zoned region at these times; hence they appear somewhat diffused. In the double-Mach region, the main features of the flow are sharp and well resolved. Moving stagnation points SP1 and SP2, the shocks M1' and S', and the slip surface SL1 are clearly visible. The gas over-expands from stagnation point SP1, reaching a maximum velocity of about $4.2 \mathrm{~km} / \mathrm{s}$ (Mach number of about 5.4) before it is shocked by S', thereby becoming compatible with conditions behind M1'.

In effect, the double-Mach structure focuses the blast energy toward the surface, thereby greatly extending the high enthalpy flow region. For example, dynamic pressures of 600 bars, pitot pressures of 1200 to $1400 \mathrm{bars}$, and total enthalpy of nearly $10^{11} \mathrm{erg} / \mathrm{g}$ seen in the regular reflection region at $50 \mathrm{~cm}$ are extended to a ground range of $80 \mathrm{~cm}$ as a result of the doubleMach flow field. 


\section{Problem 2. Blast Wave Reflections from Ideal Surfaces}

The calculated surface-level peak overpressures of the various shocks are plotted as a function of ground range in Fig. 6. The incident overpressure at ground zero of 98.6 bars reflects to a peak value of 880 bars. The corresponding theoretical reflection factor for real air is 9.43 , which results in a theoretical reflected pressure of 932 bars (versus 880 bars for the twodimensional calculation, or 6 percent low due to the zoning). The reflected pressure curve $\mathrm{R}$ agrees very well with the experimental data of Carpenter (1974), thus indirectly confirming that the calculated incident blast wave closely simulated the experimental blast wave. Near ground zero, the shocks $\mathrm{RC} 1$ and $\mathrm{RC} 2$ are much stronger than the reflected shock $\mathrm{R}$, but they decay more rapidly. As mentioned before, calculated values for $\mathrm{RC} 1$ and $\mathrm{RC} 2$ are expected to be too large because of the sharp contact surface in the calculation.

Note in particular that the pressure range curve for shock R suffers a jolt at $49.5 \mathrm{~cm}$ (i.e., near the RR limit) and locally increases at $53 \mathrm{~cm}$-this behavior perhaps being a consequence of the arrival of the sonic point singularity and the formation of a nascent Mach stem.

In the double-Mach region, the main stagnation point SP1 decays from 290 bars at transition to a value of almost 100 bars at $80 \mathrm{~cm}$. Stagnation point SP2 and shock M1' decay rather slowly from 100 bars to 75 bars. In general the calculated peaks in the double-Mach region are in excellent agreement with the experimental data (Carpenter, 1974), even at $53 \mathrm{~cm}$, where the grid points were inadequate to resolve the double-Mach stem.

\subsection{Comparisons with DMR on Wedges}

Considerably more is known about the details of double-Mach shock structures created by plane shock reflections from wedges. Such flows have many useful features:

1. The flows are self-similar (two-dimensional Cartesian), and hence are more amenable to analysis.

2. Experimental photographic results (e.g., Schlieren, shadowgraph, and interferometric data) are available to verify code calculations.

3. The complicating effects of a rarefaction wave behind the incident shock are absent.

In addition, wedge results (e.g., reflection factors) are often used to approximate the height-of-burst case. Hence, it is useful to explore the equivalence of the DMR flow field for the wedge case corresponding to the height-of-burst case.

The double-Mach structure at a time of $270 \mu s$ (Mach stem at $80 \mathrm{~cm}$ ) from the present calculation was selected for comparison. At this time the incident shock Mach number (MI) was 5.46 with an incident shock angle of about 57 degrees. The equivalent wedge case was constructed as follows. A $500 \times 100$ two-dimensional Cartesian mesh was chosen with square zones ( $\Delta x=\Delta y=1$ unit ). The shock properties corresponding to a $M_{I}=5.46$ real air shock were continuously fed into the left side of the grid at a shock angle of 57 degrees (wedge angle of 33 degrees). The second-order Godunov scheme (with Gilmore's equation of state for real air) was then used to calculate the reflected flow field. 


\section{Problem 2. Blast Wave Reflections from Ideal Surfaces}

The results of the wedge case are shown in Fig. 7. By design, the calculations are identical at the main triple point TP1. The overall features of the wedge flow field are quite similar to the height-of-burst case (Fig. 4), considering that the wedge case was about 2.4 times more coarsely zoned. Peak pressures on the wall (SP1) were 133 bars (instantaneous value at $75.2 \mathrm{~cm}$ ) for the height-of-burst case and 122 bars for the wedge, yielding "reflection factors" of 3.8 and 3.5 , respectively.

The principal differences are the reflected shock angle and the location of triple point TP3. The reflected shock angle of 49 degrees for the height-of-burst case is considerably steeper than the 22-deg angle for the wedge case. In the height-of-burst case, the rarefaction wave behind the incident shock allows the reflected shock $\mathrm{R}$ to move upward more easily into the incident wave. This causes the second Mach stem (M2) to be more vertical and the length of the reflected shock R' to be about half the value found for the wedge case. (To elucidate these points, shocks M2 and R for the height-of-burst case are depicted as dashed lines on the wedge results.) Consequently, the distance between points SP1 and M1' (i.e., the DMR duration) is somewhat shorter in the height-of-burst case.

In summary, we may conclude that the height-of-burst case is truly non-steady, and hence not amenable to similarity analysis. The rarefaction wave behind the incident shock modifies the reflected shock angle at TP3 and thereby influences the location and shape of the second Mach stem M2, compared to the equivalent wedge case. Because peak reflected pressures and "reflection factors" are a consequence of the gasdynamic state at the main triple point, they are similar for the two cases. However, the height-of-burst "reflection factor" ( $\left.R=\Delta p_{S P 1} / \Delta p_{I}\right)$ must be based on instantaneous values (with SP1, TP1, and MI' all being at different radii from the explosion center).

\section{Conclusions}

The present calculation demonstrates that the reflection of a spherical HE-driven blast wave from a plane surface creates complex flow structures on multiple length scales. In the regular reflection region, portions of shock $\mathrm{R}$ reflect within the channel formed by the wall and the dense detonation products, thus producing additional pressure pulses on the wall. The interaction of shock R with contact surface CS and slip line SL' inviscidly generates vorticity, which leads to the formation of large-scale vortex structures (i.e., turbulent mixing) on the interface between the detonation products and the air. In the double-Mach flow structure, slip lines emanating from triple points TP1 and TP2 are directed downward. The flow is forced to turn parallel at the wall, thereby converting some of the flow kinetic energy into pressure and creating stagnation points SPI and SP2 that move with the DMR structure. This also creates a supersonic wall jet consisting of a free shear layer and a wall boundary layer.

The Reynolds number of the jet is quite large, ranging from $3 \times 10^{4}$ for this case to $10^{7}$ for large-scale explosions. Hence, one would expect strong turbulent mixing at the free shear layer; however, the wall jet in these calculations was laminar. The second-order Godunov algorithm used here is non-diffusive enough to be able to calculate the evolution of discrete vortex structures started from inviscid Kelvin-Helmholtz instabilities (Glowacki et al., 1986) if adequate zoning is used in the jet (about five times finer than that used here). The wall boundary layer was not modeled. Both effects will influence the horizontal momentum of the jet, the toeing-out of 


\section{Problem 2. Blast Wave Reflections from Ideal Surfaces}

the Mach stem, and the rotational flow of the main vortices VI and V2. Adaptive gridding and a viscous wall boundary layer capability are needed to accurately model these details.

A double-Mach shock structure appeared in this calculation at a ground range between 52.5 and $55 \mathrm{~cm}$, which was 1.5 to 3.8 degrees beyond the limit of regular reflection. We believe that the so-called persistence of regular reflection in this calculation was caused by inadequate computational zoning, whereas the persistence in experiments is due to viscous wall boundary layer effects. Adaptive gridding and a viscous wall boundary layer capability are again needed to accurately calculate such flows.

The double-Mach shock structure directs some of the blast energy toward the surface, and thereby extends the high enthalpy flow to larger ground ranges. The calculated surface-level peak pressures are in excellent agreement with experimental data at all ground ranges.

A shock-on-wedge calculation was also performed to simulate the double-Mach flow field from the height-of-burst case at $\mathrm{t}=270 \mu \mathrm{s}$ (Mach stem at $80 \mathrm{~cm}$ ). Overall features of the flow were quite similar in both cases. The principal differences were the reflected shock angle, which was larger in the height-of-burst case, and the location of triple point TP3, which was closer to TPI in the height-of-burst case. These effects were attributed to the incident wave rarefaction effects and true non-steadiness of the height-of-burst case.

\section{References}

[1] S. Ando, I. I. Glass, (1981) Domains and boundaries of pseudo-stationary oblique-shackwave reflections in carbon dioxide. Proceedings of the 7th International Symposium on the Military Applications of Blast Simulation, pp. 3.6-1 to 3.6-24. Defence Research Establishment, Suffield, Ralston, Alberta, Canada, V.I.

[2] A. I. Anisimov, Ya. B. Zel'dovich, N. A. Inogamov, M. F. Ivanov, (1983) The Taylor instability of contact boundary between detonation products and a surrounding gas. Shock Waves, Explosions and Detonations, edited by J.R. Bowen et al. Progress in Astronautics and Aeronautics, 87, AAIA, New York.

[3] W. E. Baker (1973) Explosions in Air, University of Texas Press, Austin, Texas, pp. 118-149.

[4] T. V. Bazhenova, L. G. Gvozdeva, M. A. Nettleton (1984) Unsteady interactions of shock waves. Progress Aerospace Sciences 21, pp. 249-331.

[5] G. Ben-Dor I. I. and Glass (1978) Non-stationary oblique-shock-wave reflections: Actual isopycnics and numerical experiments. AlAA J. 16(11), pp. 1146-1153.

[6] G. Ben-Dor, I. I. Glass (1979) Domains and boundaries of non-stationary oblique-shockwave reflexions. 1. Diatomic gas. J. Fluid Mech. 92 (12), pp. 459-496.

[7] M. Berger, P. Colella (1986) Local adaptive mesh refinement for shock hydrodynamics $J$. Comp. Phys. 82(1) pp. 64-84.

[8] B. F. Bertrand (1972) Measurement of pressure on Mach reflection of strong shock waves in a shock tube. Mun. Report 2196, Ballistics Research Laboratory, Aberdeen, Md.

[9] W. Bleackney, A. H. Taub (1949) Interaction of shock waves . Rev. Mod. Phys. 21, pp. 584605. 


\section{Problem 2. Blast Wave Reflections from Ideal Surfaces}

[10] H. L. Brode (1955) Numerical solutions of spherical blast waves. J. Appl. Phys. 26(6), pp. 766-775.

[11] H. L. Brode (1959) Blast wave from a spherical charge, Phys. Fluids 2(2), pp. 217-229.

[12] H. J. Carpenter, D. J. Michalski, Eight-lb PBX9404 HOB Experiments, Vol. 1: Experimental Description and Data Analysis, DNA-TR-94-129-V1, Defense Nuclear Agency, 1995.

[13] P. Colella, H. M. Glaz (1984) Numerical computation of complex shock reflections in gases. Proceedings of the 9th International Conference on Numerical Methods in Fluid Dynamics, Saclay, France, edited by F. Soubbaramayer and J.P. Boujot. Springer-Verlag, Berlin.

[14] P. Colella, H. M. Glaz (1985) Efficient solution algorithms for the Riemann problem for real gases. J. Comp. Phys. 59(2), 264-289.

[15] Colella, P., Glaz, H.M., and Ferguson, R.E. (1986) Eulerian multi-material transport calculations using the second-order Godunov scheme and SLIC (in press).

[16] P. Colella, P. R. Woodward (1984) The piecewise parabolic method (PPM) for gasdynamical simulations. J. Comp. Phys. 54(1), pp. 174-201.

[17] R.Courant, K. O. Friedrichs (1948) Supersonic Flow and Shock Waves. Wiley, New York.

[18] R. L. Deschambault, I. I. Glass (1983) An update on non-stationary oblique-shock-wave reflections: Actual isopycnics and numerical experiments. J. Fluid Mech. 131, pp. 27-57.

[19] B. Dobratz (1974) Properties of chemical explosives and explosive simulants. UCRL-51319, University of California-Lawrence Livermore Laboratory, Livermore, Calif.

[20] Fry, M., Picone, J.M., Boris, J.P., and Book, D.L. (1981) Transition to double-Mach stem for nuclear explosion at 104-ft height of burst. NRL Memorandum Report 4630, Naval Research Laboratory, Washington, D.C.

[21] F. R. Gilmore (1955) Equilibrium composition and thermodynamic properties of air to 24000 K, RM-1543, Rand Corporation, Santa Monica, Calif.

[22] I. I. Glass (1982) private communication, UTIAS, University of Toronto, Toronto, Canada.

[23] H. M. Glaz (1979) Development of random choice numerical methods for blast wave problems. NSWC/NOL TR 78-211, Naval Surface Weapons Center, White Oak, Md.

[24] H. M. Glaz, P. Colella, I. I. Glass, R. L. Deschambault (1985a) A numerical study of oblique shock-wave reflections with experimental comparisons. Proc. R. Soc. London, Ser. A, 398, pp. 117-140.

[25] H. M. Glaz, P. Colella, I. I. Glass, R. L. Deschambault (1985b) A detailed numerical, graphical, and experimental study of oblique shock wave reflections. UTIAS Report 285, University of Toronto, Toronto, Canada.

[26] H. M. Glaz, I. I. Glass, J. C. J. Hu, P. Walter (1986) Oblique shock wave reflections in SF6: A comparison of calculation and experiment. $10^{\text {th }}$ International Colloquium on the Dynamics of Explosions and Reactive Systems August 4-9, 1985, Berkeley, California (to be published by AlAA).

[27] W. J. Glowacki, A. L. Kuhl, H. M. Glaz, R. E. Ferguson (1986) Shock wave interactions with high sound speed layers, Proceedings of the $20^{\text {th }}$ Shock Tube Symposium, 29 July-2 Aug, 1985, Berkeley, California (forthcoming). 


\section{Problem 2. Blast Wave Reflections from Ideal Surfaces}

[28] C. F. Hansen (1959) Approximations for the thermodynamic and transport properties of high-temperature air, NASA TR R-50.

[29] L. F. Henderson, A. Lozzi (1975) Experiments on transition of Mach reflexion, Part 1. J. Fluid Mech. 68, pp. 139-155.

[30] H. Hornung (1985) Regular and Mach reflection of shock waves. Ann. Rev. Fluid Mech. 18, pp. 33-58.

[31] H. G. Hornung, J. R. Taylor (1982) Transition from regular to Mach reflection of shock waves, Part 1: The effect of viscosity in the pseudo-steady case, J. Fluid Mech. 123, pp. 143153.

[32] J. T. C. Hu, I. I. Glass (1986) An interferometric and numerical study of pseudo-stationary oblique-shock-wave reflections in sulfur hexafluoride (SF6), Proc. R. Soc. London (to be published).

[33] A. L. Kuhl (1982) private communication. R\&D Associates, Marina del Rey, Calif.

[34] A. L. Kuhl, M. R. Seizew (1978) Analysis of ideal, strong Chapman-Jouguet detonations, TRW Tech. Rept. 78.4735, 9-1, TRW Systems, Redondo Beach, Calif.

[35] V. P. Korobeinikov, P. I. Chushkin (1966) Plane, cylindrical, and spherical blast waves in a gas with counter-pressure, Proceedings of the Steklov Institute of Mathematics, Akademiya Nauk Moscow, SSSR, pp. 4-33.

[36] J. H. Lee, I. I. Glass (1984) Domains and boundaries of pseudo-stationary oblique shockwave reflections in air, UTIAS Report 262, University of Toronto, Toronto, Canada; see also, Prog. Aerosp. Sci. 21, pp. 53-80.

[37] W. F. Noh (1976) Numerical methods in hydrodynamic calculations, UCRL-52112, Lawrence Livermore National Laboratory, Livermore, Calif.

[38] Noh, W.F., and Woodward, P.R. (1976) SLIC (simple line interface calculation) Proceedings of the $5^{\text {th }}$ International Conference on Numerical Methods in Fluid Dynamics, 28 June-3 July 1976, Enschede, Netherlands, edited by A.I. Van der Vooren and P.J. Zandbergen, pp. 330-340, Springer-Verlag, Berlin.

[39] D. F. Okhotsimskii, I. L. Kondrasheva, Z. P. Vlasova, R. K. Kazakova R.K. (1957)

Transactions of the Steklov Mathematics Institute, 50. Akademiya Nauk, Moscow, SSSR.

[40] A. K. Oppenheim, A. L. Kuhl, E. A. Lundstrum, M. M. Kamel (1972a) A parametric study of self-similar blast waves. J. Fluid Mech. 52(4), pp. 657-682.

[41] A. K. Oppenheim, A. L. Kuhl, M. M Kamel (1972b) On self-similar blast waves headed by the Chapman-Jouguet detonation, J. Fluid Mech. 55(2), pp. 257-270.

[42] L. I. Sedov (1946) Propagation of strong blast waves, Prikl. Mat. Mech. 10, pp. 241-250.

[43] L. I. Sedov (1959) Similarity and Dimensional Methods in Mechanics. Academic Press, New York.

[44] M. Shirouzu, I. I. Glass (1982) An assessment of recent results on pseudo-stationary oblique-shock-wave reflections. UTIAS Report 264, University of Toronto, Toronto, Canada.

[45] L. V. Shurshalov (1978) Two-dimensional calculations of an explosion in the real atmosphere, Arch. Mech. 30(4-5), pp. 629-643. 


\section{Problem 2. Blast Wave Reflections from Ideal Surfaces}

[46] G. I. Taylor (1941) The formation of a blast wave by a very intense explosion. British Report RC-210 (1941), reviewed in Proc. R. Soc. London, Ser. A, 201 (1) (1950), pp. 175186.

[47] G. I. Taylor (1950) The dynamics of the combustion products behind plane and spherical detonation fronts in explosives. Proc. R. Soc. London, Ser. A, 200(1), pp. 235-247.

[48] J. Von Neumann, H. Goldstine (1955) Blast wave calculation. Comm. on Pure and Appl. Math. 8, pp. 327-353.

[49] J. Von Neumann, R. D. Richtmeyer (1950) A method for the numerical calculations of hydrodynamic shocks. J. Appl. Phys. 21, pp. 232-7. 
Problem 2. Blast Wave Reflections from Ideal Surfaces
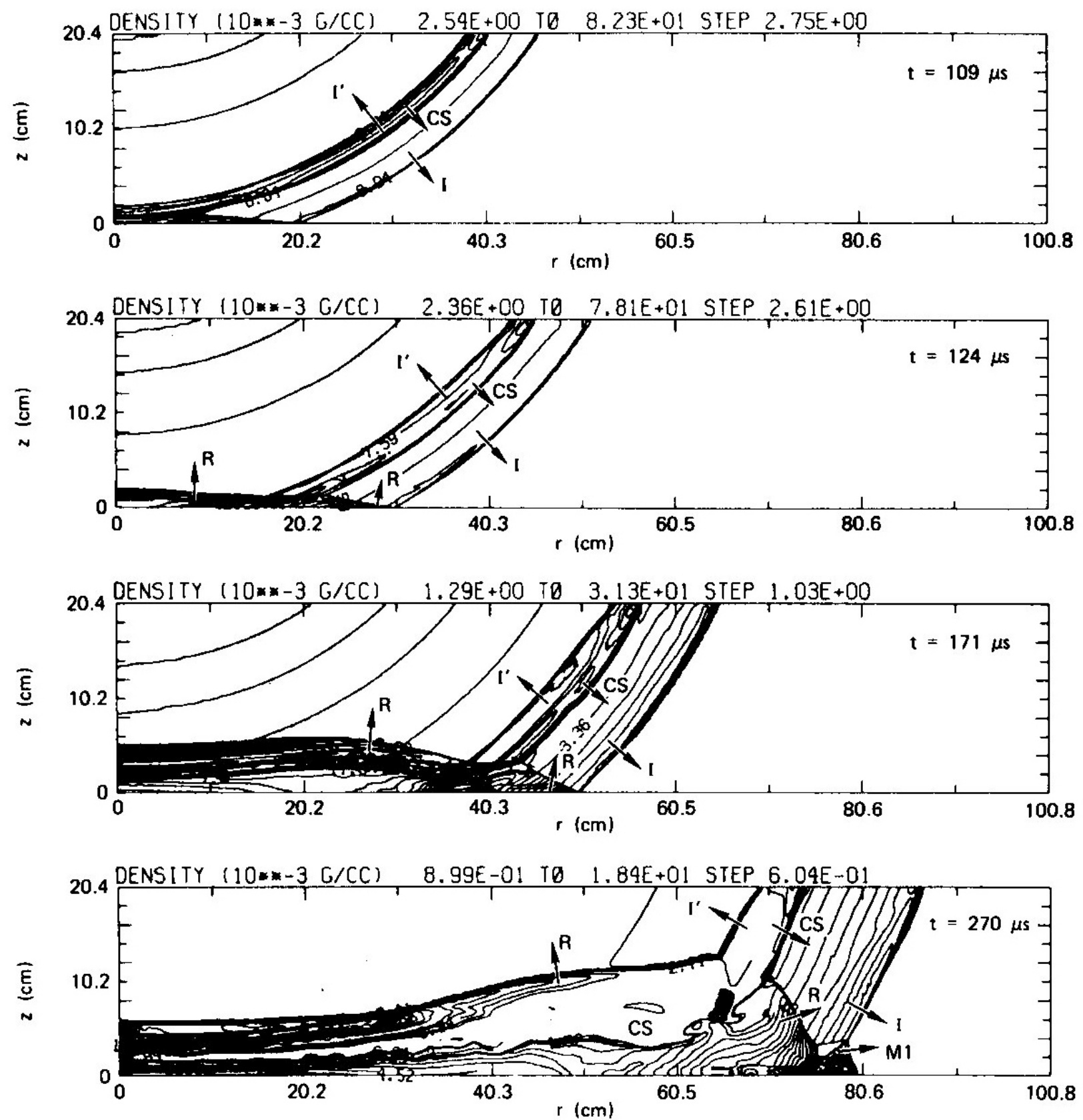

Figure 1a. Transition from regular to double-Mach reflection for a spherical, HE-driven blast wave reflecting from an ideal plane surface. Density contours $\left(\mathrm{mg} / \mathrm{cm}^{3}\right)$. 
Problem 2. Blast Wave Reflections from Ideal Surfaces
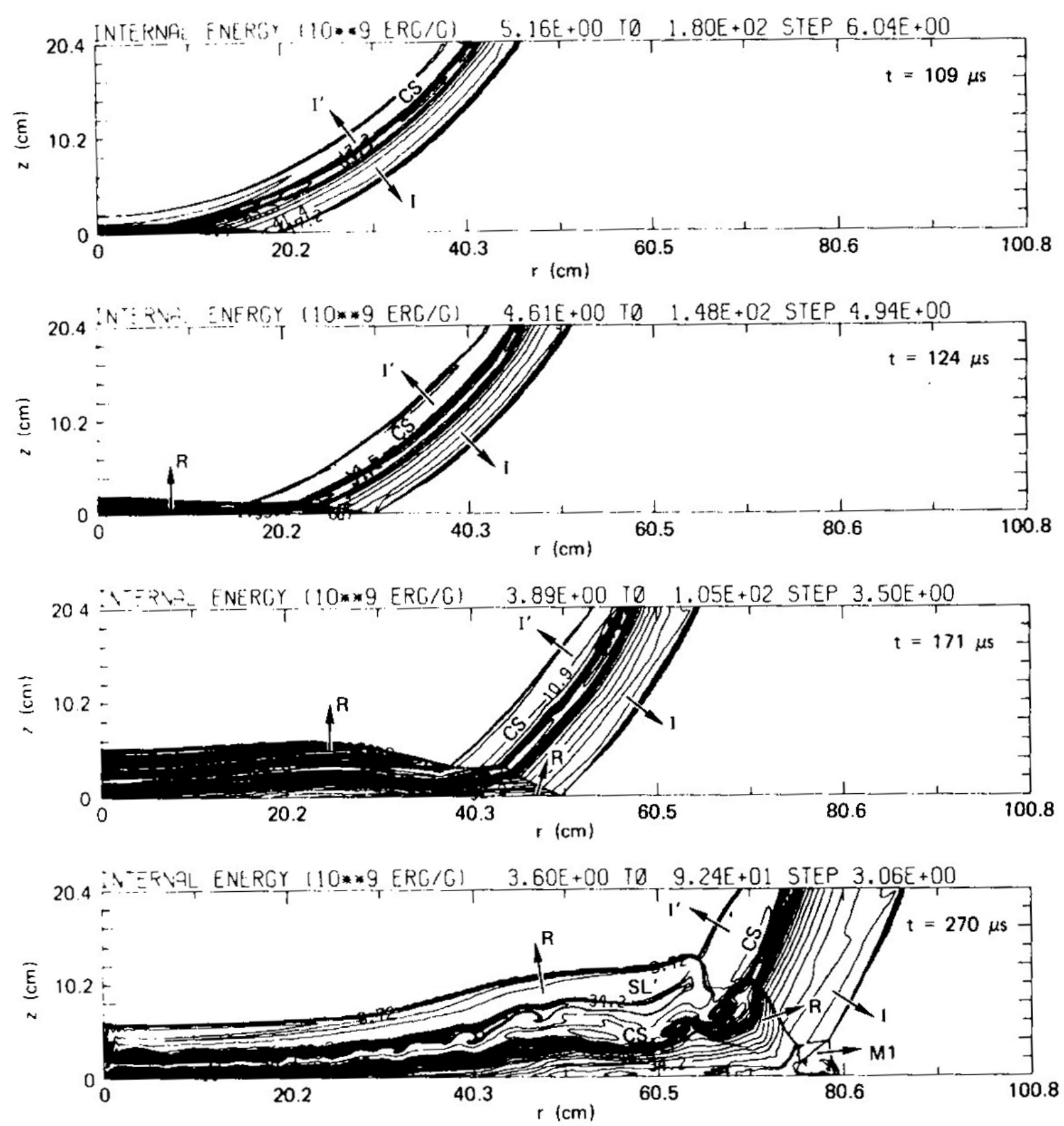

Figure 1b. Transition from regular to double-Mach reflection for a spherical, HE-driven blast wave reflecting from an ideal plane surface. Internal energy contours $\left(10^{9} \mathrm{erg} / \mathrm{g}\right)$. 
Problem 2. Blast Wave Reflections from Ideal Surfaces
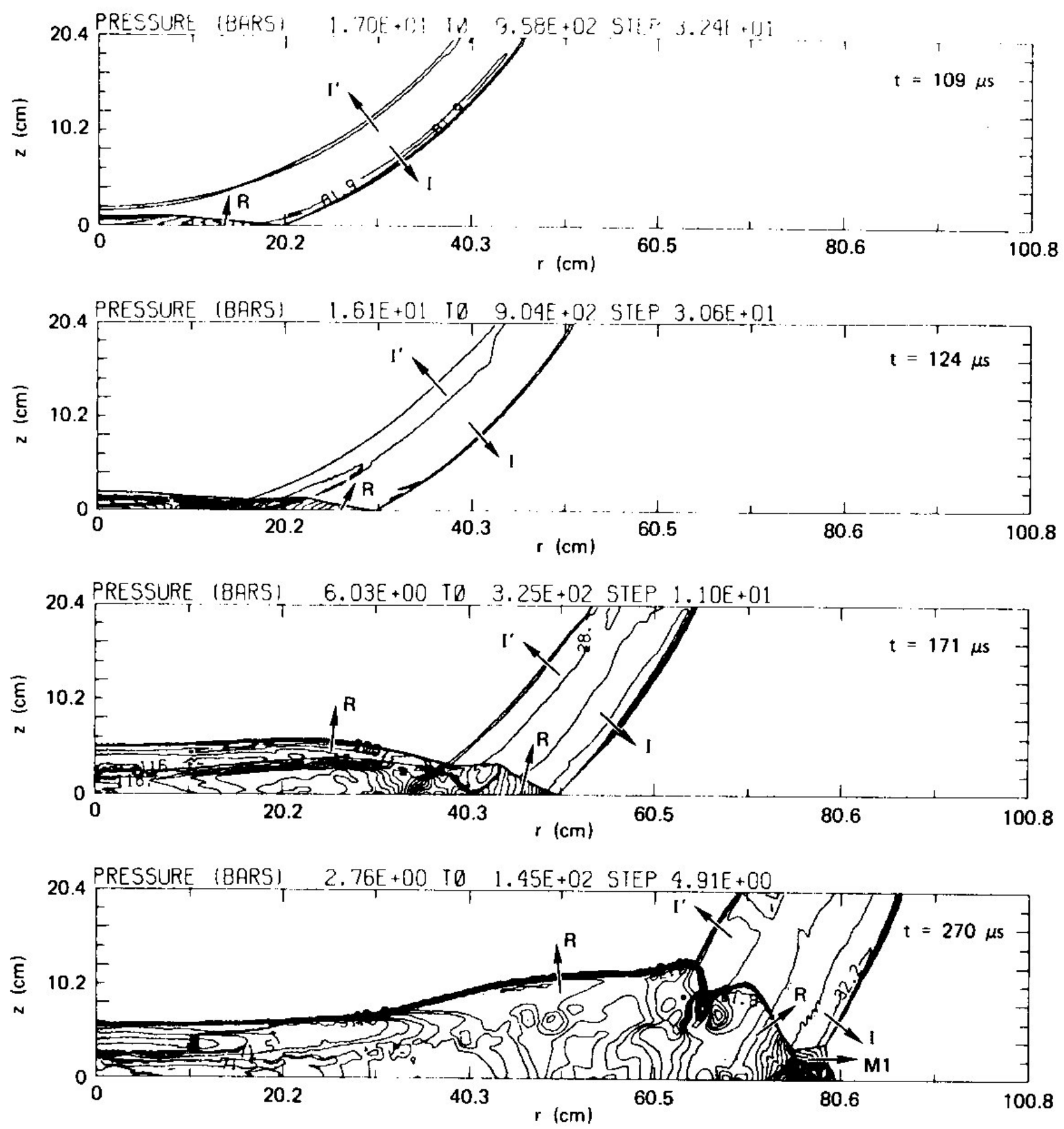

Figure 1c. Transition from regular to double-Mach reflection for a spherical, HE-driven blast wave reflecting from an ideal plane surface. Pressure contours (bar). 
Problem 2. Blast Wave Reflections from Ideal Surfaces
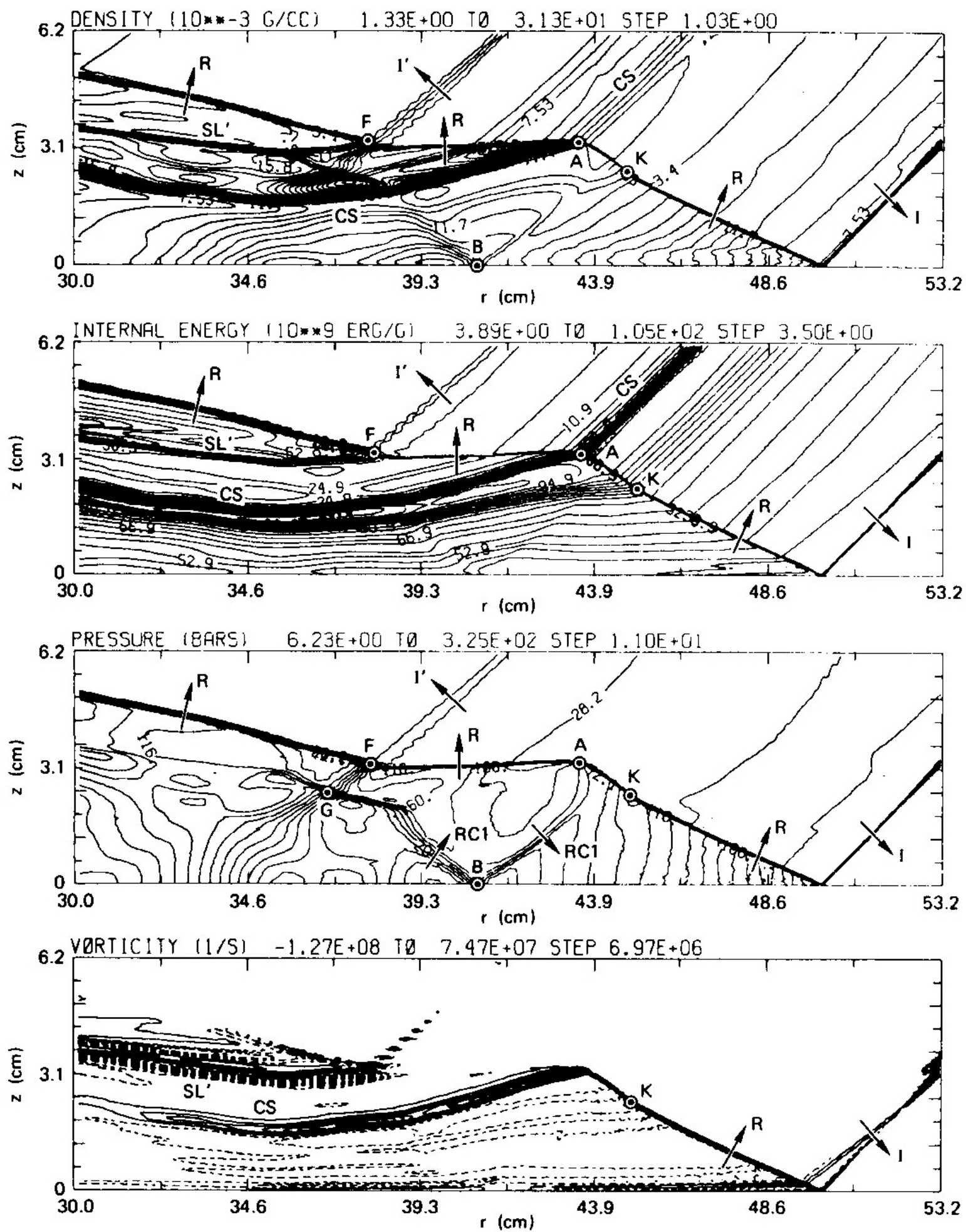

Figure 2a. Interaction of the reflected wave R with the contact surface CS and shock I' in the regular reflection regime $(t=171 \mu \mathrm{s}$; reflection point at $50 \mathrm{~cm})$. 
Problem 2. Blast Wave Reflections from Ideal Surfaces
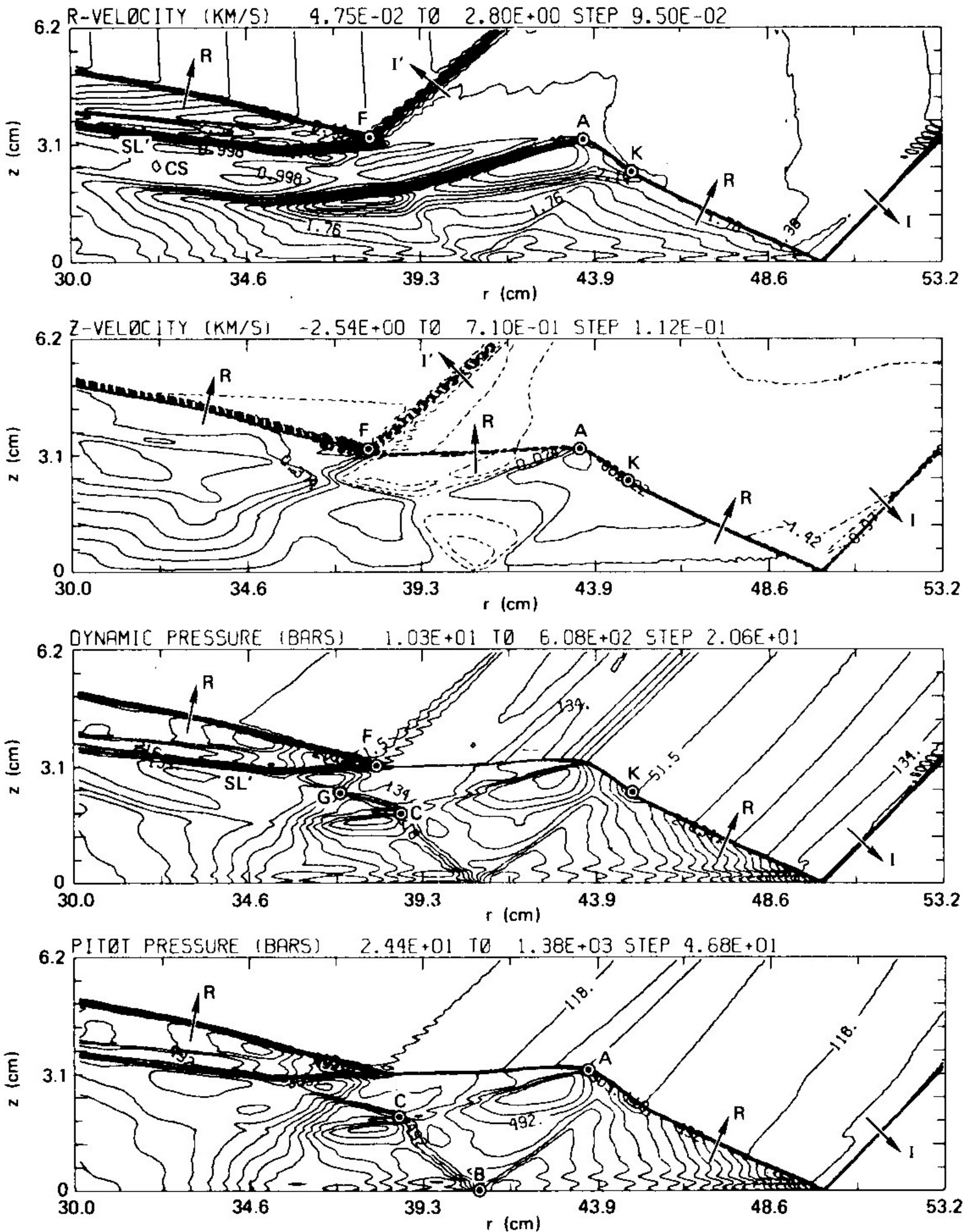

Figure 2b. Interaction of the reflected wave $\mathrm{R}$ with the contact surface CS and shock I' in the regular reflection regime $(t=171 \mu \mathrm{s}$; reflection point at $50 \mathrm{~cm})$. 
Problem 2. Blast Wave Reflections from Ideal Surfaces
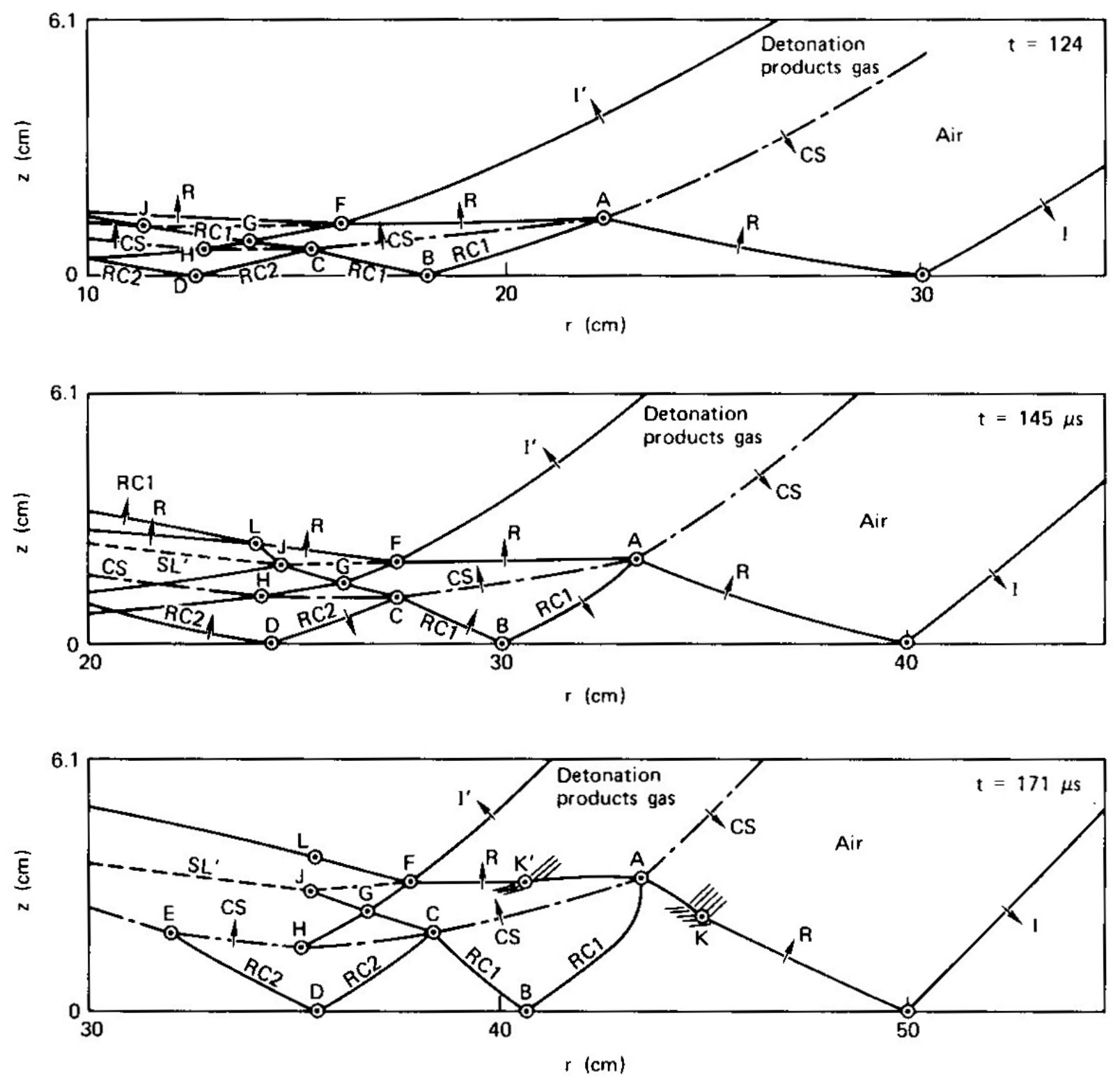

Figure 2c. Interaction of the reflected wave R with the contact surface CS and shock I' in the regular reflection regime. Schematic showing wave interactions (a) $t=124 \mu \mathrm{s}, r=30 \mathrm{~cm}$, (b) $t=145 \mu \mathrm{s}, r=40 \mathrm{~cm}$, (c) $t=171 \mu \mathrm{s}, r=50 \mathrm{~cm}$. 
Problem 2. Blast Wave Reflections from Ideal Surfaces
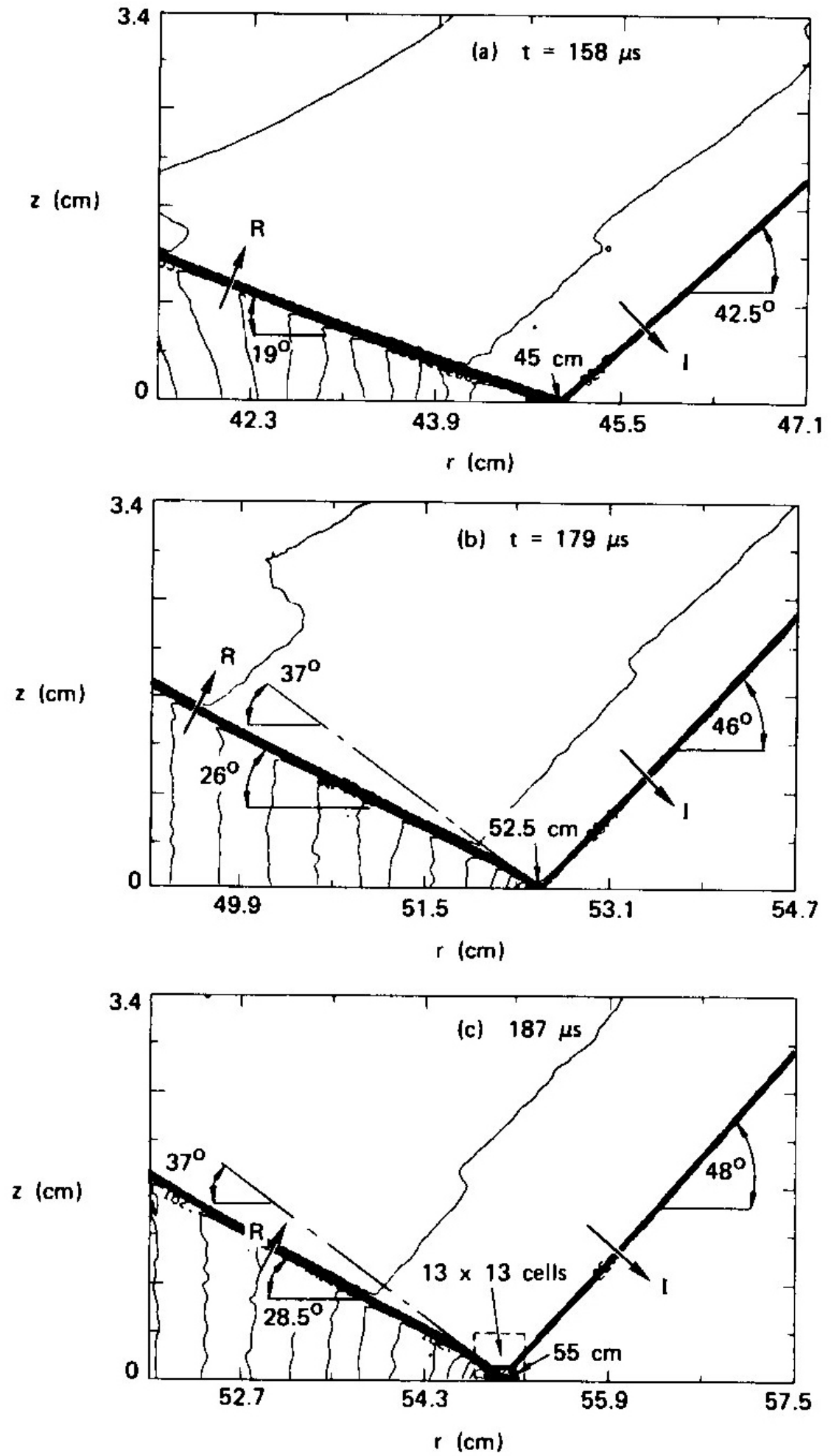

Figure 3. Pressure contours showing transition from regular to double-Mach reflection: (a) $t=158 \mu \mathrm{s}, r=45 \mathrm{~cm}$ (RR), (b) $t=179 \mu \mathrm{s}, r=52.5 \mathrm{~cm}$ (PRR), (c) $t=187 \mu \mathrm{s}, r=53 \mathrm{~cm}$ (DMR). 
Problem 2. Blast Wave Reflections from Ideal Surfaces
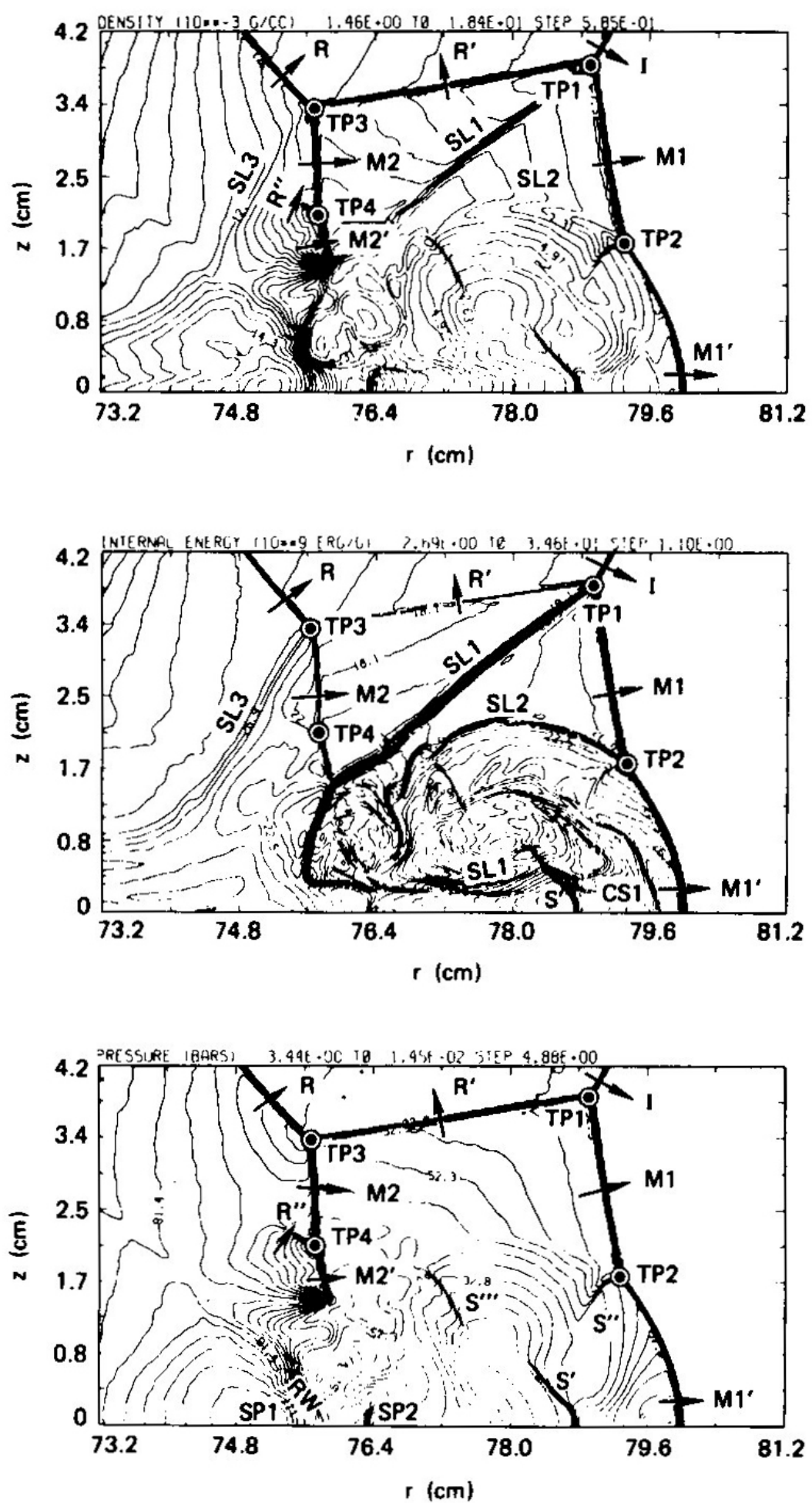

Figure 4a. Shock structure in the double-Mach reflection regime $(t=270 \mu \mathrm{s}$, Mach stem at 80 $\mathrm{cm})$. 
Problem 2. Blast Wave Reflections from Ideal Surfaces
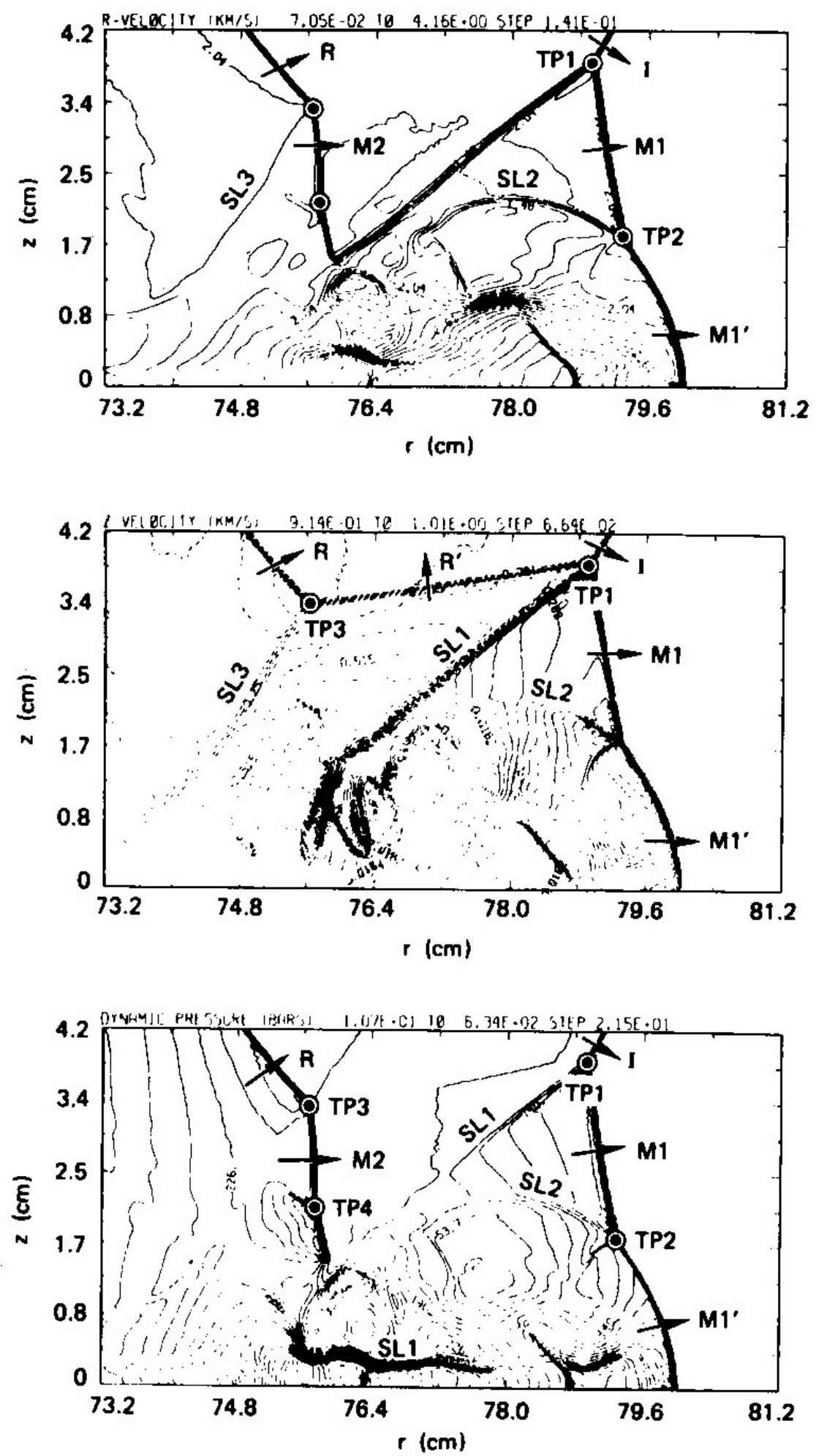

Figure 4b. Shock structure in the double-Mach reflection regime $(t=270 \mu s$, Mach stem at 80 $\mathrm{cm})$. 
Problem 2. Blast Wave Reflections from Ideal Surfaces
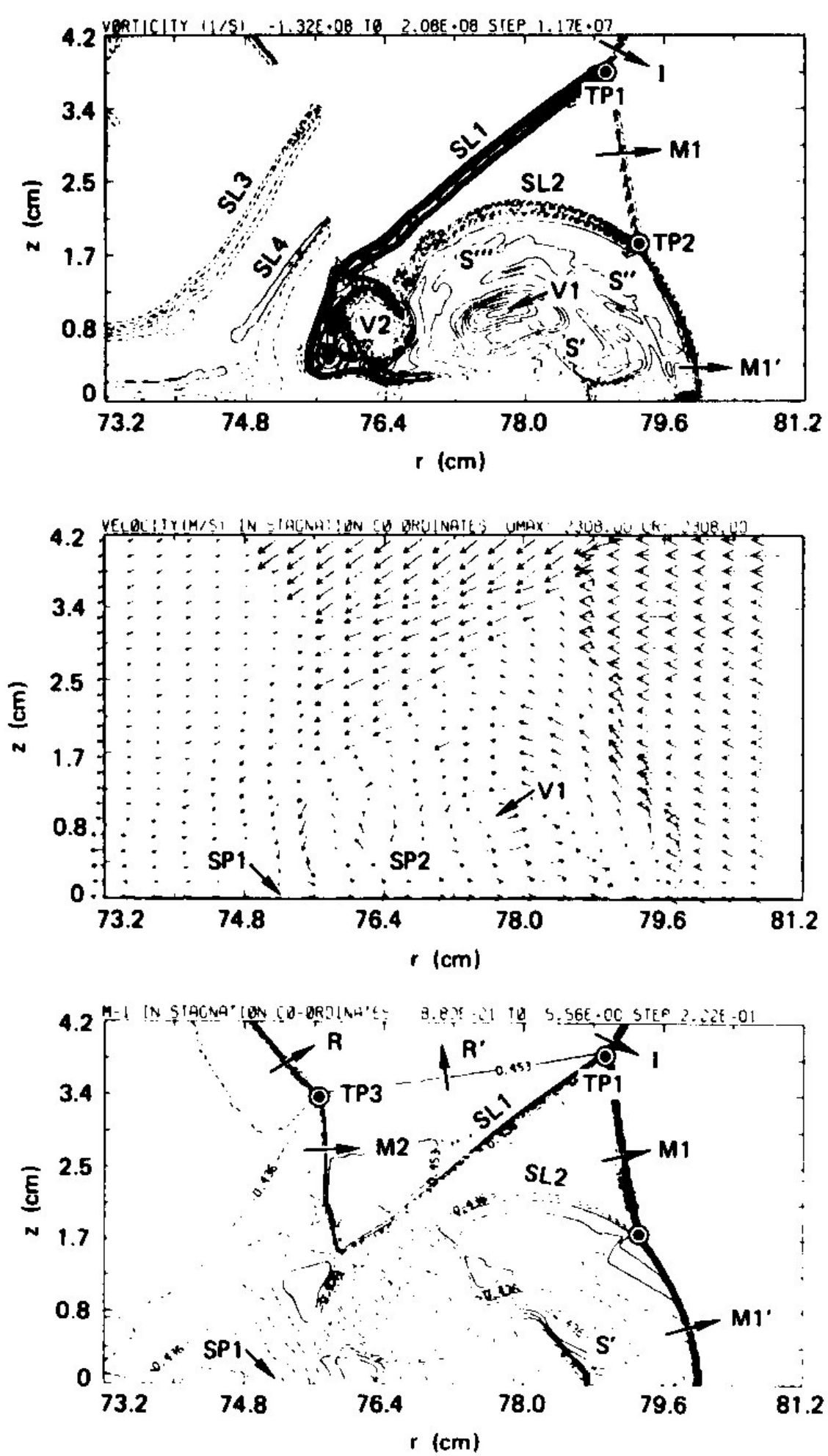

Figure 4c. Shock structure in the double-Mach reflection regime $(t=270 \mu s$, Mach stem at 80 $\mathrm{cm})$. 


\section{Problem 2. Blast Wave Reflections from Ideal Surfaces}
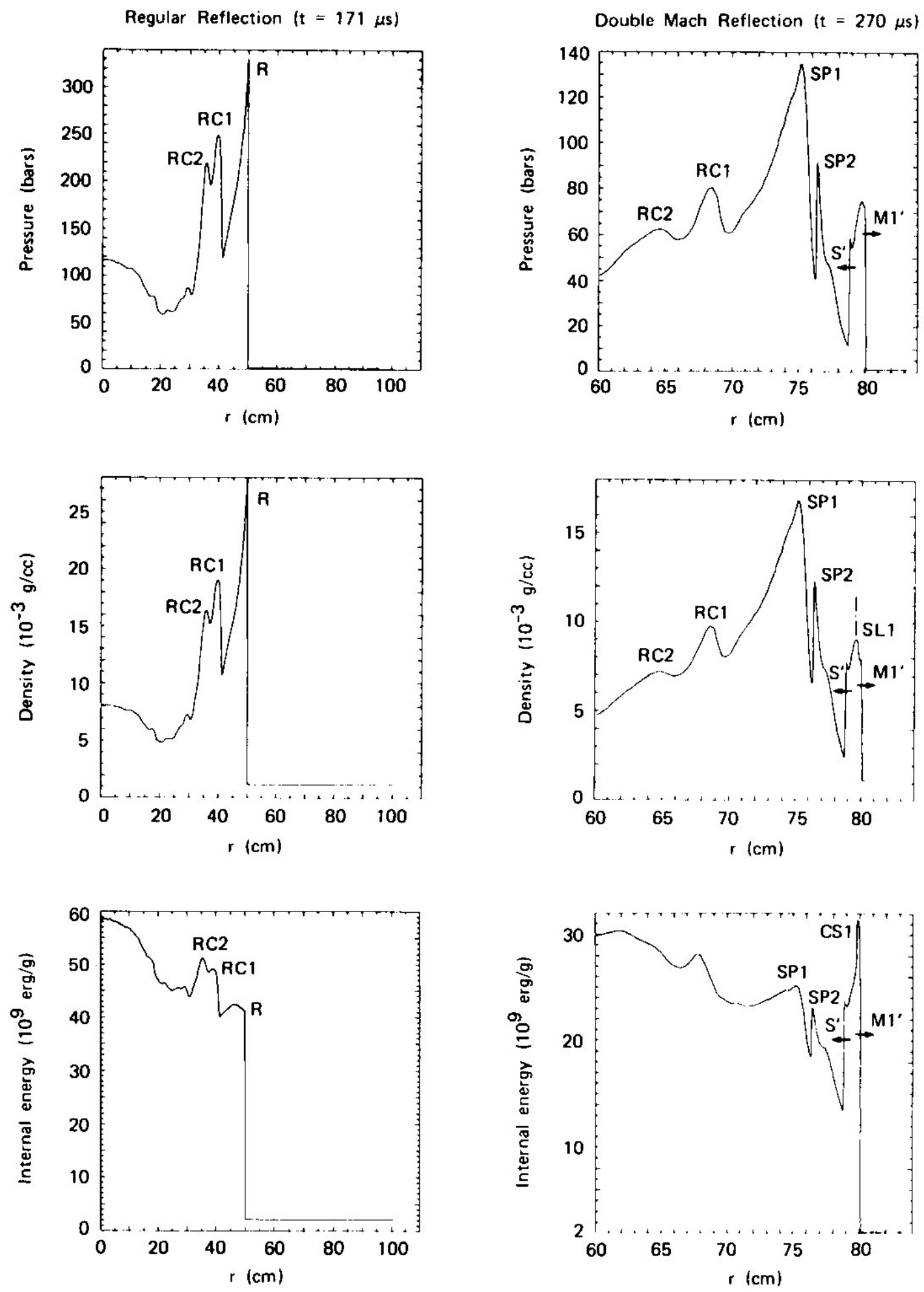

Figure 5a. Comparison of surface-level flow fields in the regular reflection regime ( $t=171 \mu \mathrm{s})$ and double-Mach reflection regime $(t=270 \mu s)$ 
Problem 2. Blast Wave Reflections from Ideal Surfaces
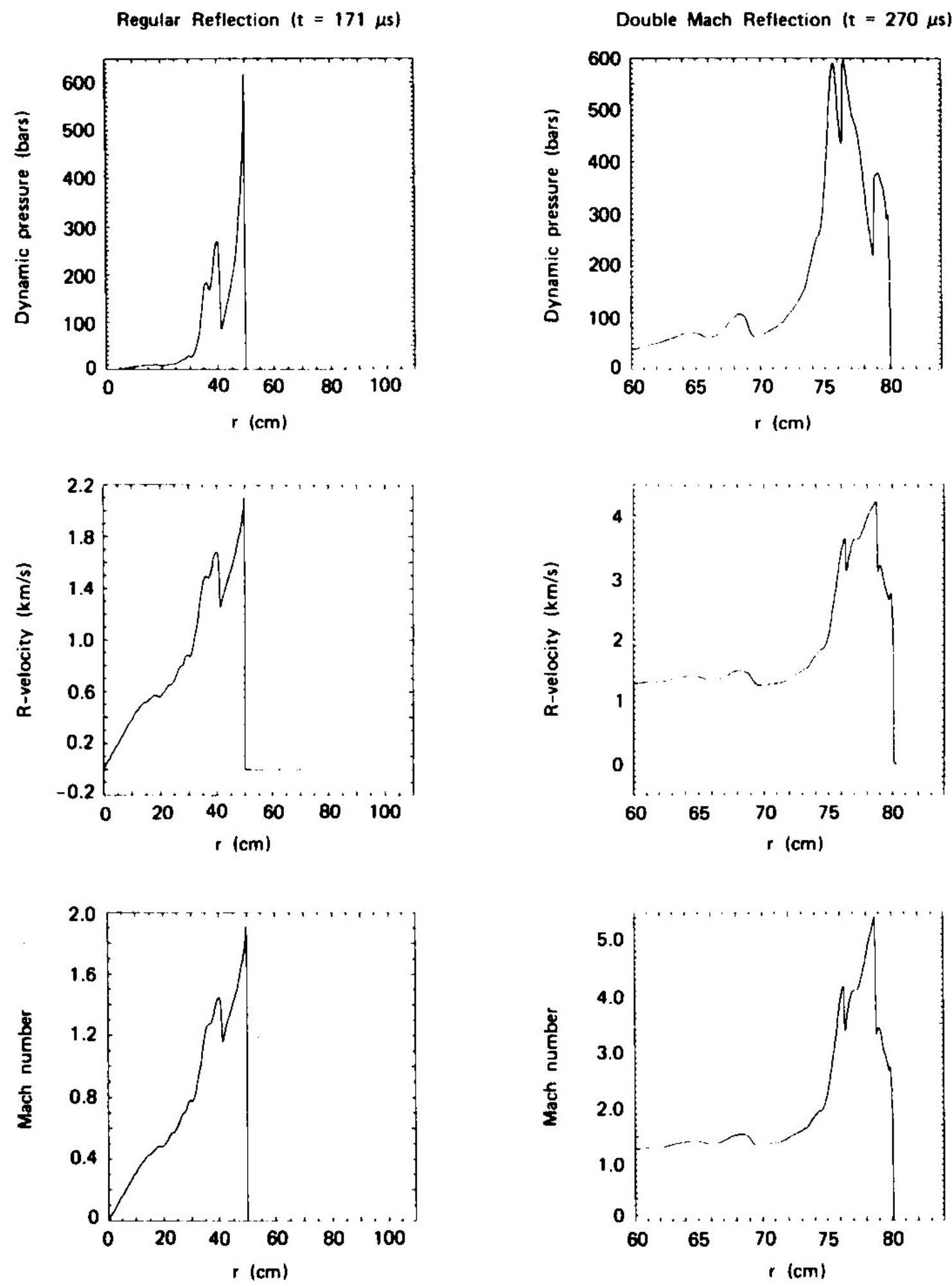

Figure 5b. Comparison of surface-level flow fields in the regular reflection regime ( $t=171 \mu \mathrm{s})$ and double-Mach reflection regime $(t=270 \mu s)$. 
Problem 2. Blast Wave Reflections from Ideal Surfaces
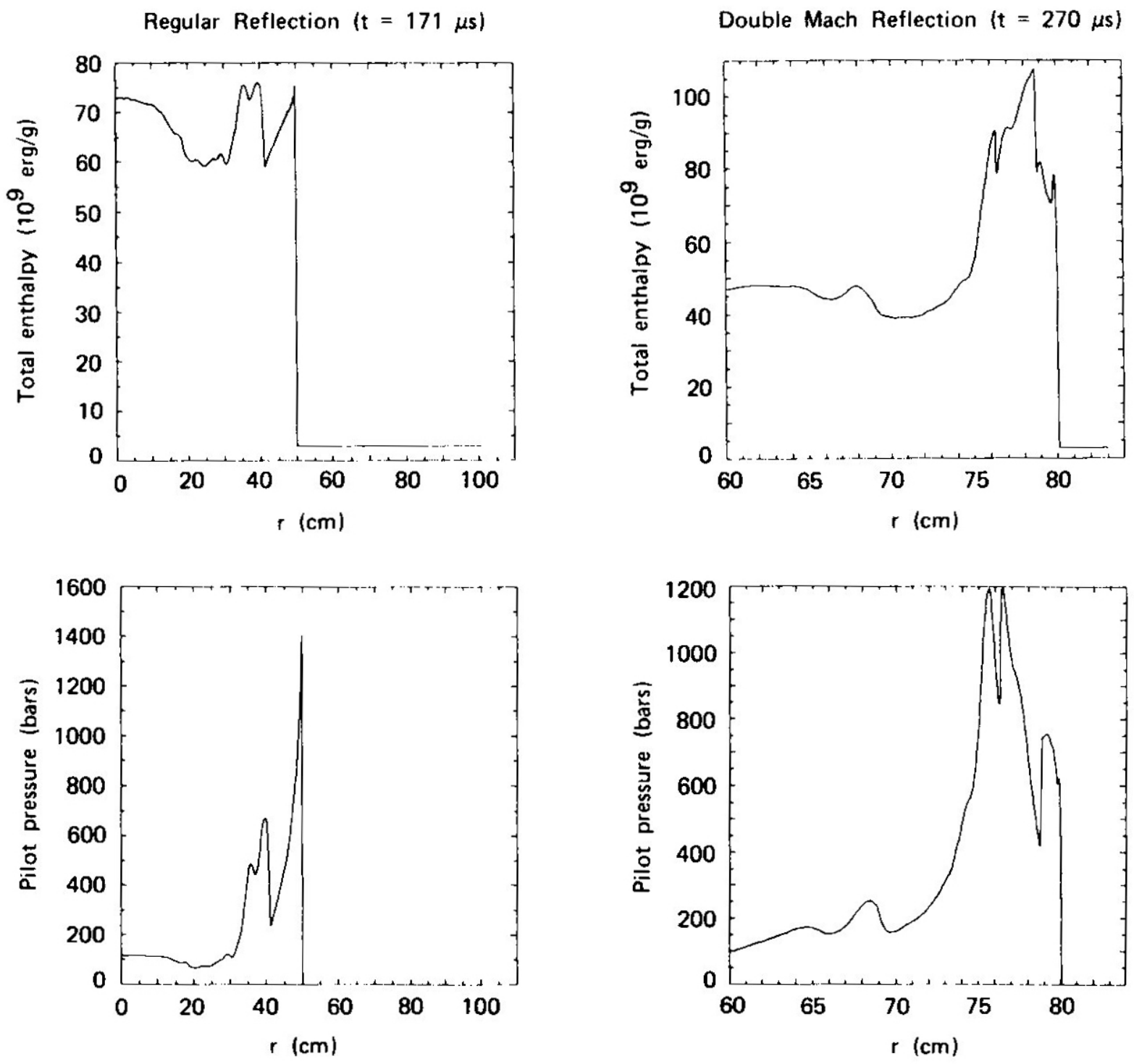

Figure 5c. Comparison of surface-level flow fields in the regular reflection regime ( $t=171 \mu \mathrm{s})$ and double-Mach reflection regime $(t=270 \mu s)$ 
Problem 2. Blast Wave Reflections from Ideal Surfaces

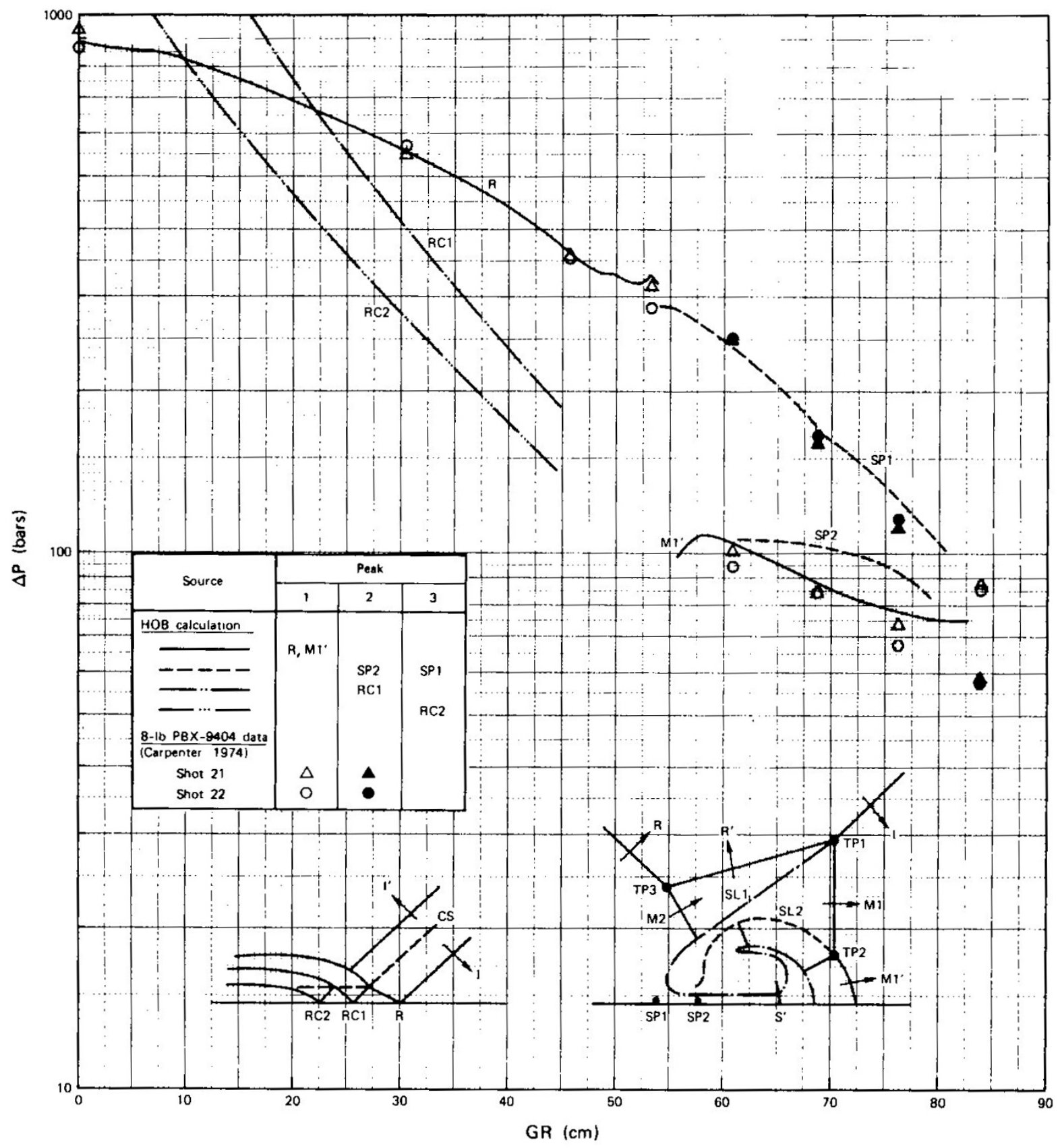

Figure 6. Comparison of calculated peak pressures on the surface with experimental data (Carpenter, 1974). 
Problem 2. Blast Wave Reflections from Ideal Surfaces

\subsection{Assessment}
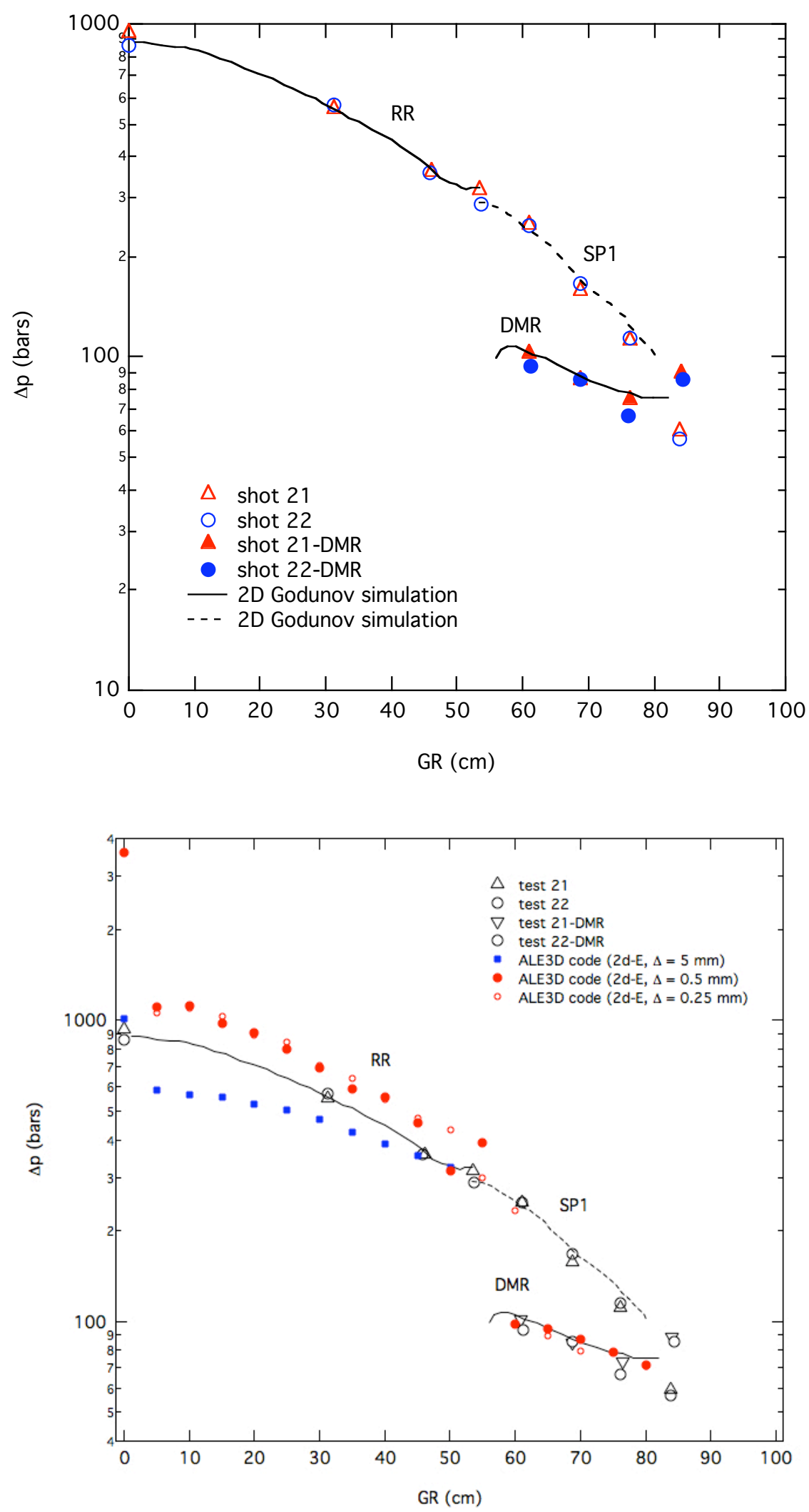


\section{Problem 2. Blast Wave Reflections from Ideal Surfaces}

Table 3-1. Test matrix, atmospheric conditions, and scaling factors for 8-1b PBX-9404 spheres.

\begin{tabular}{|c|c|c|c|c|c|c|c|c|c|c|}
\hline $\begin{array}{l}\text { Test } \\
\text { No. }\end{array}$ & $\begin{array}{l}\text { Test } \\
\text { date }\end{array}$ & $\begin{array}{c}\mathrm{HOB} \\
(\mathrm{ft})\end{array}$ & $\begin{array}{c}P_{0} \\
\text { (in. } \mathrm{Hg} \text { ) }\end{array}$ & $\begin{array}{l}\mathrm{T}_{0} \\
\left.{ }^{\circ} \mathrm{C}\right)\end{array}$ & $S_{p}$ & $\mathrm{~S}_{\mathrm{d}}$ & $S_{t}$ & $\mathrm{~S}_{\mathrm{i}}$ & $\begin{array}{c}\text { SHOB } \\
\text { (ft) }\end{array}$ & $\begin{array}{c}\text { SHOB } \\
\left(\mathrm{ft} / \mathrm{KT}^{1 / 3}\right) \\
\end{array}$ \\
\hline 24 & 19 Oct. 1973 & 7.250 & 29.62 & 33.40 & 1.0102 & 0.9966 & 1.0280 & 1.0384 & 7.226 & 338 \\
\hline 6 & 20 Aug. 1973 & 6.750 & 29.53 & 24.30 & 1.0132 & 0.9956 & 1.0116 & 1.0250 & 6.720 & 315 \\
\hline $\begin{array}{l}4 \\
5 \\
\end{array}$ & $\begin{array}{l}14 \text { Aug. } 1973 \\
\text { 16 Aug. } 1973 \\
\end{array}$ & $\begin{array}{l}6.350 \\
6.350 \\
\end{array}$ & $\begin{array}{l}29.39 \\
29.48 \\
\end{array}$ & $\begin{array}{l}29.20 \\
27.78 \\
\end{array}$ & & & & & $\begin{array}{l}6.312 \\
6.319 \\
\end{array}$ & $\begin{array}{l}295 \\
296 \\
\end{array}$ \\
\hline $\begin{array}{l}1 \\
2 \\
3 \\
\end{array}$ & $\begin{array}{l}18 \text { Jul. } 1973 \\
19 \text { Jul. } 1973 \\
25 \text { Jul. } 1973 \\
\end{array}$ & $\begin{array}{l}5.933 \\
5.933 \\
5.933 \\
\end{array}$ & $\begin{array}{l}29.56 \\
29.44 \\
29.41 \\
\end{array}$ & $\begin{array}{l}27.50 \\
16.67 \\
29.50 \\
\end{array}$ & & & & & $\begin{array}{l}5.909 \\
5.901 \\
5.899 \\
\end{array}$ & $\begin{array}{l}277 \\
277 \\
276 \\
\end{array}$ \\
\hline $\begin{array}{l}7 \\
8 \\
\end{array}$ & $\begin{array}{l}22 \text { Aug. } 1973 \\
23 \text { Aug. } 1973 \\
\end{array}$ & $\begin{array}{l}5.080 \\
5.080 \\
\end{array}$ & & $\begin{array}{l}28.60 \\
28.60 \\
\end{array}$ & & & & & $\begin{array}{l}5.062 \\
5.062 \\
\end{array}$ & $\begin{array}{l}237 \\
237 \\
\end{array}$ \\
\hline $\begin{array}{r}9 \\
25 \\
\end{array}$ & & $\begin{array}{l}3.813 \\
3.813 \\
\end{array}$ & & $\begin{array}{l}25.00 \\
27.00 \\
\end{array}$ & & & & & $\begin{array}{l}3.796 \\
3.799 \\
\end{array}$ & $\begin{array}{l}178 \\
178 \\
\end{array}$ \\
\hline $\begin{array}{l}10 \\
11 \\
\end{array}$ & $\begin{array}{l}14 \text { Sep. } 1973 \\
17 \text { Sep. } 1973 \\
\end{array}$ & $\begin{array}{l}2.967 \\
2.967 \\
\end{array}$ & & $\begin{array}{l}20.00 \\
20.40 \\
\end{array}$ & & & & & $\begin{array}{l}2.957 \\
2.958 \\
\end{array}$ & $\begin{array}{l}140 \\
140 \\
\end{array}$ \\
\hline $\begin{array}{l}12 \\
13 \\
14 \\
\end{array}$ & $\begin{array}{l}19 \text { Sep. } 1973 \\
20 \text { Sep. } 1973 \\
21 \text { Sep. } 1973 \\
\end{array}$ & $\begin{array}{l}2.700 \\
2.700 \\
2.700 \\
\end{array}$ & $\begin{array}{l}29.66 \\
29.64 \\
29.58 \\
\end{array}$ & $\begin{array}{l}26.20 \\
24.80 \\
22.70 \\
\end{array}$ & $\begin{array}{l}1.0088 \\
1.0095 \\
1.0115 \\
\end{array}$ & & & $\begin{array}{l}1.0252 \\
1.0233 \\
1.0211 \\
\end{array}$ & $\begin{array}{l}2.692 \\
2.692 \\
2.690 \\
\end{array}$ & $\begin{array}{l}131 \\
131 \\
131 \\
\end{array}$ \\
\hline $\begin{array}{l}15 \\
16 \\
\end{array}$ & $\begin{array}{l}24 \text { Sep. } 1973 \\
25 \text { Sep. } 1973 \\
\end{array}$ & $\begin{array}{l}2.543 \\
2.543 \\
\end{array}$ & $\begin{array}{l}29.50 \\
29.46 \\
\end{array}$ & $\begin{array}{l}22.80 \\
26.00 \\
\end{array}$ & & & & & $\begin{array}{l}2.531 \\
2.530 \\
\end{array}$ & $\begin{array}{l}123 \\
123 \\
\end{array}$ \\
\hline $\begin{array}{l}17 \\
18 \\
19 \\
\end{array}$ & $\begin{array}{l}1 \text { Oct. } 1973 \\
3 \text { Oct. } 1973 \\
5 \text { Oct. } 1973 \\
\end{array}$ & $\begin{array}{l}2.118 \\
2.118 \\
2.118 \\
\end{array}$ & $\begin{array}{l}29.60 \\
29.55 \\
29.53\end{array}$ & $\begin{array}{l}24.40 \\
26.80 \\
20.60\end{array}$ & $\begin{array}{l}1.0108 \\
1.0126 \\
1.0132 \\
\end{array}$ & $\begin{array}{l}0.9964 \\
0.9958 \\
0.9956 \\
\end{array}$ & & $\begin{array}{l}1.0235 \\
1.0288 \\
1.0186 \\
\end{array}$ & $\begin{array}{l}2.110 \\
2.109 \\
2.109\end{array}$ & $\begin{array}{l}107 \\
107 \\
107\end{array}$ \\
\hline $\begin{array}{l}20 \\
21 \\
\end{array}$ & $\begin{array}{l}8 \text { Oct. } 1973 \\
9 \text { Oct. } 1973 \\
\end{array}$ & $\begin{array}{l}1.695 \\
1.695 \\
\end{array}$ & $\begin{array}{l}29.50 \\
29.52 \\
\end{array}$ & $\begin{array}{l}17.80 \\
23.00 \\
\end{array}$ & $\begin{array}{l}.0143 \\
1.0136 \\
\end{array}$ & $\begin{array}{l}0.9953 \\
0.9955 \\
\end{array}$ & $\begin{array}{l}1.0001 \\
1.0092 \\
\end{array}$ & $\begin{array}{l}1.0144 \\
1.0230 \\
\end{array}$ & $\begin{array}{l}1.687 \\
1.687 \\
\end{array}$ & $\begin{array}{l}92.6 \\
92.6 \\
\end{array}$ \\
\hline $\begin{array}{l}22 \\
26 \\
\end{array}$ & $\begin{array}{l}11 \text { Oct. } 1973 \\
30 \text { Oct. } 1973 \\
\end{array}$ & $\begin{array}{l}1.272 \\
1.272 \\
\end{array}$ & $\begin{array}{l}29.50 \\
29.70 \\
\end{array}$ & $\begin{array}{l}24.60 \\
32.00 \\
\end{array}$ & $\begin{array}{l}1.0143 \\
1.0074 \\
\end{array}$ & $\begin{array}{l}0.9953 \\
0.9975 \\
\end{array}$ & $\begin{array}{l}1.0117 \\
1.0266 \\
\end{array}$ & $\begin{array}{l}1.0262 \\
1.0342 \\
\end{array}$ & $\begin{array}{l}1.266 \\
1.269 \\
\end{array}$ & $\begin{array}{l}74.6 \\
74.8 \\
\end{array}$ \\
\hline $\begin{array}{l}23 \\
27 \\
28\end{array}$ & $\begin{array}{l}12 \text { Oct. } 1973 \\
5 \text { Nov. } 1973 \\
6 \text { Nov. } 1973\end{array}$ & $\begin{array}{l}0.848 \\
0.848 \\
0.848\end{array}$ & $\begin{array}{l}29.52 \\
29.75 \\
29.79\end{array}$ & $\begin{array}{l}27.00 \\
28.80 \\
22.40\end{array}$ & $\begin{array}{l}1.0136 \\
1.0057 \\
1.0044\end{array}$ & $\begin{array}{l}0.9955 \\
0.9981 \\
0.9985\end{array}$ & $\begin{array}{l}1.0160 \\
1.0217 \\
1.0113\end{array}$ & $\begin{array}{l}1.0298 \\
1.0276 \\
1.0157\end{array}$ & $\begin{array}{l}0.844 \\
0.846 \\
0.847\end{array}$ & $\begin{array}{l}- \\
57.2 \\
57.2\end{array}$ \\
\hline
\end{tabular}




\title{
Problem 3: Blast Wave Reflections from Ground Surfaces ${ }^{11}$
}

\begin{abstract}
Numerical simulations of the turbulent dusty flow induced by an explosion over a ground surface were performed with a second-order Godunov code. Adaptive Mesh Refinement (AMR) was used to capture the convective mixing processes on the computational grid. The most prominent feature of the flow was a turbulent wall jet that was induced by the precursor shock refraction into a thermal layer on the surface. Dust lofting occurred because the rotational structures of the boundary layer entrained dust from the ground surface. Scaling methods were defined to evaluate the mean and root-mean-squared profiles of the wall jet. The profiles were shown to be similar to the profiles calculated for self-similar wall jets propagating over dust layers, and agreed with experimental profiles of a dusty boundary layer behind a shock. The Convective Mixing Model described here can be applied to a wide spectrum of fluid-dynamic mixing problems.
\end{abstract}

\section{Introduction}

The problem considered here is the turbulent dusty boundary layer induced by a point explosion over a ground surface. As will be demonstrated, the fluid-dynamic aspects of this problem are quite spectacular and are certainly worth detailed scientific study. On a more practical note, knowledge of such boundary layers is important to explosion safety analysis (e.g., to calculate drag loads on structures). Although much is known about the blast wave environments from two-dimensional gasdynamic code calculations by Colella et al. (1986) and experiments of Reichenbach et al. (1992), very little is known quantitatively about the turbulent boundary layer flow embedded in explosions - both because experimental measurements are extremely difficult and because current turbulence models are inadequate for such two-phase flows. In this paper we attempt to quantify our knowledge of the dusty flow induced by a point explosion by means of direct numerical calculations of the convective mixing on an adaptive grid.

Perhaps the most prominent fluid-dynamic feature of the flow is a turbulent wall jet that is induced by a precursor shock structure. During a point explosion, thermal energy from the fireball heats the air near the ground surface, creating a thermal layer ahead of the shock front. The blast wave propagates faster in this high-sound-speed layer, and forms a characteristic precursor shock structure. Figure 1 presents a shadow photograph of a blast wave precursor

${ }^{11}$ Kuhl, A. L. (1993) Turbulent wall jet in a blast wave precursor (plenary lecture), 1993 Japanese National Symposium on Shock Wave Phenomena (K. Takayama Ed.), Tohoku Print, Sendai, Japan. Also published as UCRL-JC-112713, Lawrence Livermore National Laboratory, Livermore, CA, 40 pp. 


\section{Problem 3. Blast Wave Reflections from Ground Surfaces}

observed in laboratory experiments (Reichenbach, 1985) at the Ernst-Mach Institut, Freiburg, Germany. The blast wave was created by the detonation of an 0.5 -g Nitropenta charge at 6.7 $\mathrm{cm}$ above the surface. The thermal layer was simulated by a high-sound-speed layer, formed by forcing helium through the porous floor of the test chamber. Clearly visible are the various waves associated with a precursor shock structure: the precursor shock $P$ and turning shock $T$, the Mach stem $M$ and its associated slip lines $\mathrm{SL}_{\mathrm{P}}$ and $\mathrm{SL}_{\mathrm{TP}}$, the incident shock $I$, and the boundary layer BL. More important, the photograph graphically demonstrates that the wall jet embedded in the precursor flow is turbulent.

Turbulent mixing is always driven by the vorticity field. One of the unique features of dusty flows is that vorticity is created by an inviscid mechanism, namely, baroclinic effects (in contrast to clean boundary layers that are created by viscous no-slip boundary conditions). This is perhaps best illustrated by considering the inviscid vorticity transport equation:

$$
\frac{\partial}{\partial t} \boldsymbol{\omega}+(\mathbf{u} \cdot \nabla) \boldsymbol{\omega}=(\boldsymbol{\omega} \cdot \nabla) \mathbf{u}-\boldsymbol{\omega}(\nabla \cdot \mathbf{u})-\nabla(1 / \rho) \times \nabla p+\nabla \times(\mathbf{B} / \rho)
$$

The terms on the left-hand side represent the time rate-of-change of the vorticity vector $\boldsymbol{\omega}$, and the convection of vorticity by the velocity field $\mathbf{u}$. The vorticity transport is balanced by a number of source terms on the right-hand side: I - vorticity stretching along streamlines; II

- vorticity dilatation due to compressibility; III- vorticity creation by baroclinic effects (i.e., pressure gradients interacting with oblique density gradients); and IV - vorticity creation by body forces B. Missing on the right-hand side is the term $\nu \nabla^{2} \boldsymbol{\omega}$ that represents the diffusion of vorticity by molecular transport effects. This is consistent with our convective mixing approximation to turbulent flows at high Reynolds numbers.

Baroclinic effects predominate during the blast wave phase of the explosion. Vorticity is created by shock interactions with strong density gradients (e.g., the dust layer, thermal layer and fireball). These shear layers are hydrodynamically unstable, and rapidly transition into turbulent mixing layers. The rotational structures in the boundary layer entrain dust from the surface. Turbulent mixing and transport of the dust then occurs via the velocity field induced by the vorticity distribution. Additional vorticity generation occurs both because of large-scale pressure gradients in the blast wave, and because of local pressure gradients induced by the rotational structures in the mixing layer. Buoyancy effects dominate the cloud rise phase.

While others have used numerical models based on the mean-flow equations and turbulence closure models (Barthel 1985; Donaldson et al. 1982; Traci and Su 1988), here we propose a Convective Mixing Model (Kuhl 1992) for flows with large density variations (e.g., $10^{5}$ density variations between the dust layer and the fireball gases) that actually calculates the turbulent mixing and transport of dust on an adaptive grid. This approach has been used to calculate dusty boundary layers behind a normal shock and various reflected shock structures, and dusty boundary layers embedded in self-similar wall jet flows such as a doubleMach reflection on a dusty wedge and a square wave precursor (Kuhl et al. 1990, 1993, 1991 


\section{Problem 3. Blast Wave Reflections from Ground Surfaces}

and 1989). The present calculations then represent the first extension of this methodology to a truly non-self-similar problem, namely, a blast wave flow.

The formulation of the calculations is described in the next section. The results include: a flow visualization of the evolution of the wall jet, quantification of the dust layer growth versus time, scaling methods for analyzing the turbulent flow, and statistical averaging to establish the mean and root-mean-squared (r.m.s.) profiles across the mixing layer. Finally, the present results are compared with other self-similar wall jet flows and data.

\section{Formulation of the Calculations}

The numerical calculations were made with a Convective Mixing Model that has been proven to be effective in simulating turbulent flows with strong baroclinic effects (Kuhl 1992). The model is based on the following idealizations: (1) a loose dust bed; (2) an instantaneous shock fluidization of the dust layer; (3) the dust and air are in local equilibrium, hence, air viscosity enforces the no-slip condition between phases; (4) the dust-air mixture is treated as a continuum dense fluid with zero viscosity; (5) the turbulent transport is dominated by baroclinically-generated vorticity and convective mixing effects; and (6) a two-dimensional flow approximation is adequate to capture the major features of the mixing. These assumptions lead to an inviscid set of conservation laws for the mixture:

$$
\begin{aligned}
& \frac{\partial}{\partial t} \rho+\nabla \cdot(\rho \mathbf{u})=0 \\
& \frac{\partial}{\partial t} \rho \mathbf{u}+\nabla(\rho \mathbf{u u})=-\nabla p \\
& \frac{\partial}{\partial t} \rho E+\nabla \cdot(\rho E \mathbf{u})=-\nabla \cdot(p \mathbf{u})
\end{aligned}
$$

where $\mathbf{u}$ denotes the velocity and $E$ represents the total energy: $E=e+0.5 \mathbf{u} \cdot \mathbf{u}$. The pressure $p$ is related to the density $\rho$ and internal energy $e$ by the equation of state

$$
p=\left(\gamma_{e}-1\right) \rho e
$$

where $\gamma_{e}$ denotes the effective $\gamma$ of the mixture. Note that this model has a particle pressure in the dense fluidized bed and allows slip at the bottom of the bed. Both effects are consistent with observations of granular flow in dense fluidized beds. In the above equations, $\rho$ actually represents the mixture density. The dust density $\rho_{d}$ may be calculated from the relation

$$
\rho_{d}=\rho C
$$

This requires an additional transport equation for the dust concentration $C$, namely:

$$
\frac{\partial}{\partial t} C+(\mathbf{u} \cdot \nabla) C=0
$$

while the gas density $\rho_{g}$ may be evaluated from mass conservation: $\rho=\rho_{g}+\rho_{d}$. The above set of equations were integrated by means of a high-order Godunov algorithm for dusty 


\section{Problem 3. Blast Wave Reflections from Ground Surfaces}

gasdynamics (Colella and Glaz 1985; Glaz et al. 1993). AMR (Berger and Colella 1989) was used to capture the convective mixing processes of the turbulent flow.

An $r-z$ cylindrical coordinates grid was used for the computational mesh. Three levels of mesh refinement were utilized: grid 1 defined the computational domain of $2000 \mathrm{~m}$ in height by $2000 \mathrm{~m}$ in radius, grid 2 was used to capture the shock waves, while grid 3 was reserved for tracking the dusty flow. A mesh refinement study was performed to check the convergence of the solution. Mesh sizes are listed in Table 1.

Table 1. Mesh Refinement Studies

\begin{tabular}{|c|c|c|c|c|c|c|c|}
\hline \multirow{2}{*}{ Case } & \multicolumn{2}{|c|}{ CPU (hrs) } & \multirow{2}{*}{$\begin{array}{l}\Delta_{1} \\
(\mathrm{~m})\end{array}$} & \multirow{2}{*}{$\begin{array}{l}\Delta_{2} \\
(\mathrm{~m})\end{array}$} & \multirow{2}{*}{$\begin{array}{l}\Delta_{3} \\
(\mathrm{~m})\end{array}$} & \multirow{2}{*}{$\begin{array}{c}\Delta_{3} \\
\mathrm{~cm} / \mathrm{KT}^{1 / 3}\end{array}$} & \multirow{2}{*}{$\begin{array}{c}\mathrm{n} \\
\text { cells }\end{array}$} \\
\hline & $\mathrm{t}=1 \mathrm{~s}$ & $2.5 \mathrm{~s}$ & & & & & \\
\hline 1 - coarse & $0.8^{+}$ & $2^{+}$ & 62.50 & 10.42 & 1.736 & 50 & 2 \\
\hline 2 - medium & $10.0^{+}$ & $\sim 42^{+}$ & 31.25 & 5.21 & 0.868 & 25 & 4 \\
\hline 3 - fine & $10.5^{*}$ & $\sim 33^{*}$ & 15.63 & 2.60 & 0.434 & 12.5 & 8 \\
\hline 4 - very fine & $91.5^{*}$ & - & 7.813 & 1.30 & 0.217 & 6.25 & 16 \\
\hline
\end{tabular}

The computational grid was initialized with conditions corresponding to a U.S. standard atmosphere assuming a ground plane located at $900 \mathrm{~m}$ altitude. The ambient conditions at ground level were:

$$
\begin{aligned}
& p_{a}=0.910 \times 10^{6} \mathrm{dy} / \mathrm{cm}^{2}, \quad \rho_{a}=1.12 \times 10^{-3} \mathrm{~g} / \mathrm{cm}^{3} \\
& e_{a}=2.03 \times 10^{9} \mathrm{erg} / \mathrm{g}, \quad \text { and } a_{a}=\sqrt{\gamma p_{a} / \rho_{a}}=0.337 \times 10^{5} \mathrm{~cm} / \mathrm{s}
\end{aligned}
$$

Next, a dust layer was mapped into the bottom of the grid. It consisted of $n$ cells of soil at a $\rho_{s}=1000 \mathrm{mg} / \mathrm{cm}^{3}, n$ cells of fluidized bed at a $\rho_{\mathrm{FB}}=50 \mathrm{mg} / \mathrm{cm}^{3}$, and $n$ cells of a dust gradient region where the dust density decreased linearly from $50 \mathrm{mg} / \mathrm{cm}^{3}$ to 0 . Then a thermal layer of $n$ cells was placed on top of the dust layer. The thermal layer had a uniform density profile in the vertical direction but varied with ground range to give the effective sound speed as inferred from experimental arrival-time measurements (Carpenter et al. 1991). Note that the thickness $H$ of each of the layers remained constant $\left(H=n \Delta_{3}=\right.$ $3.472 \mathrm{~m}=1 \mathrm{~m} / \mathrm{KT}^{1 / 3}$ ) in all calculations, while the number of cells $n$ in each layer increased with grid refinement. To start the problem, the flow field corresponding to a $37-\mathrm{KT}$ explosion at $t=52 \mathrm{~ms}$ was placed at $203 \mathrm{~m}$ above the ground plane (see Fig. 2).

The left-hand boundary of the grid was treated as an axis of symmetry. The bottom boundary was treated as an inviscid slip boundary. The top and right boundaries were treated as outflow boundaries. 


\section{Problem 3. Blast Wave Reflections from Ground Surfaces}

The calculations were run to a time of 1 second. For case 4, this required about 92 CPU hours on the Cray YMP; timings for other cases are listed in Table 1. The results are described in the next section.

\section{Flow Visualization}

The initial conditions for the calculation were depicted in Figure 2, which showed the incident Shock $I$ and blast wave at $t=51.56 \mathrm{~ms}$, and the dust layers $D L$ and thermal layer $T L$. Figure 3 presents an overall view of the flowfield at late times $(t=1027 \mathrm{~ms})$. It also introduces a color scheme that will be used to depict the flowfield in subsequent figures. For example, variations in gas density are made visible by a color scale (Fig. $3 \mathrm{~b}$ ): blue represents ambient air density, green denotes shock-compressed air, red corresponds to shock-heated air, and the fireball gases are coded yellow. A separate grey scale (Fig. 3c) is used to visualize the dust density. In addition, pressure contours are shown as black lines, while white dashed lines depict the medium and fine grid patches.

At this time, the incident shock $I$ and Mach stem $M$ have expanded to a ground range of about $800 \mathrm{~m}$. The fireball (FB) is no longer spherical; the reflected shock $R$ deposited vorticity as it passed through the fireball and caused shock-torusing well before any bulk rise of the fireball has occurred as a result of buoyancy forces. Also visible are the precursor shock $P$ and dusty boundary layer (BL) along the surface. The next series of figures present the details of the evolution of the precursor and boundary layer flow from the very-fine zoned calculation (Case 4) - spanning the time between Figures 2 and 3.

Figure 4 shows the formation of the precursor shock $P$, which started to outrun the incident shock at a ground range $G R \simeq 130 \mathrm{~m}$. In the regular-reflection regime $(G R<200 \mathrm{~m})$, the thermal layer gas seems to accumulate under the precursor shock (Fig. 4a). The wall jet (WJ) does not fully form until a range of $G R \simeq 240 \mathrm{~m}$ (Fig. $4 \mathrm{~b}$ ), which is well beyond the regular reflection region. From inception, the wall jet is turbulent. The wall jet leaves behind a vorticity layer that rapidly rolls up into a dusty boundary layer (Fig. 4c). Back near ground zero, the dust layer actually grows by a Richtmyer-Meshkov mechanism (Fig. 4a), forming vertical fingers or striations. Also evident is shock $T$ (actually a continuation of the incident shock $I$ ), which turns the precursor-shocked gas flowing over the top of the wall jet back toward the surface. This partially stagnates the flow, generating reflected shocks $R^{\prime}$ and $R$. The secondary reflected shock $R^{\prime \prime}$ is created when the dust layer stops compressing and the flow is forced to turn parallel to the surface.

In the next series of figures (i.e., Figs. 5 through 9), flow visualization includes not only black-line pressure contours for visualizing rarefaction waves, but also vorticity contours (positive shown as as red lines and negative as white lines) for visualizing shear layers and black-line contours of dilatation (i.e., $\nabla \cdot \mathbf{u}$ ) which identify shocks. Figures 5 and 6 depict the growth of the precursor and the formation of the Mach stem shock $M$, as required by shock theory (i.e., when the angle between shocks $I$ and $P$ becomes large enough). Close examination of the vorticity contours shows that vorticity is generated by baroclinic effects when the precursor shock interacts with the top of the thermal layer (creating the free shear 


\section{Problem 3. Blast Wave Reflections from Ground Surfaces}

layer FSL) and the top of the dust layer (creating the boundary layer BL). These two shear layers rapidly become unstable, roll up, and strongly interact to form the turbulent wall jet. These interactions lead to the mixing of dust throughout the wall jet flow. Some of the vorticity in the dust layer falls behind the wall jet to form the dusty boundary layer. Counter-sign vorticity (i.e., red contours) is baroclinically generated there by entrainment of dust from the fluidized bed. Also visible are the sliplines emitting from the Mach stem triple points. The dilatation contours highlight the main shock structures and the weak acoustic waves radiating from the turbulent mixing structures of the wall jet.

Figures 7 and 8 show the continued propagation of the precursor and the evolution of the jet. Large-scale merging of vortex structures has finally caused a mixing structure to break off from the rear of the wall jet. These figures also show that dust entrainment occurs in long striations in between vortex structures, and thus, dust scouring is very localized and intermittent.

By a time of $916 \mathrm{~ms}$ (Fig. 9), large-scale merging processes have begun to break up the jet. Continued merging of vortex structures causes the wall jet to stop. The wall jet gases then balloon upward to form the dust pedestal DP at a range of about 800 meters. No doubt the details of the jet breakup are three-dimensional and dependent on the grid resolution of the calculation.

Figure 10 depicts the complete flowfield at $t=1027 \mathrm{~ms}$. The dust heave region is visible back near ground zero ( 0 to $300 \mathrm{~m})$. Actually it is not a boundary layer scouring phenomenon - rather, it is formed by a decompression of the dust layer. It can be thought of as a sound speed effect, where the rarefaction waves in the air relax much more quickly than in the dust layer. This creates a pressure gradient that heaves the dust layer upward. Initially the mixing layer grows by a Richtmyer-Meshkov mechanism (Fig. 4a), and there is very little gas flow above the layer. Later, the updraft toward the axis and core of the fireball deforms the vertical striations into a Kelvin-Helmholtz-type mixing layer. Some of the dust from the top of the heave layer is drawn up into the fireball, and thus forms the leading edge of the dust stem $(D S)$.

\section{Dust Layer Growth}

The numerical solution was analyzed to determine the evolution of the dust layer as a function of time. We begin by defining the visual height of the dust layer $z_{v}$ as the instantaneous height where the dust concentration approaches zero. The layer height $z_{v}(r, t)$ was measured at fixed ranges, and the results are plotted as a function of time in Figure 11. The dust layer grows during the precursor phase, and reaches a peak value that increases with increasing range - because the scale of the wall jet increases. Then the dust height drops rapidly as the rear of the wall jet passes a station. The dust layer grows more slowly during the boundary layer phase, and reaches an asymptotically constant value by the end 


\section{Problem 3. Blast Wave Reflections from Ground Surfaces}

of the blast wave ( $t \simeq 2.5$ seconds). The layer is thicker at smaller ground ranges, because convective mixing processes have been transporting the dust for a longer time.

Next, let us define the mean visual dusty boundary layer thickness as:

$$
\delta_{v}(r, t)=\bar{z}_{v}(r, t)-\bar{z}_{o}(r, t)
$$

where $\bar{z}_{o}$ denotes the mean height of the dust (initially about 10 meters). The results are presented in Figure 12. During the positive phase at 500 meters, the dusty boundary layer thickness grows as a power-law function of time; during the negative phase $(t>1.6 \mathrm{~s})$ it grows more slowly. Similar power-law growths are found at 600 and 700 meters during their positive phases. This suggests that all of the data may be collapsed to a single curve. In fact, if one scales the time with the local shock arrival time $t_{a}$, one finds the following relations:

$$
\delta_{v}=5 \tau^{1.5} \quad\left[\delta_{v}\right]=\text { meters }
$$

or

$$
\delta_{v}=1.4 \tau^{1.5} \quad\left[\delta_{v}\right]=\mathrm{m} / \mathrm{KT}^{1 / 3}
$$

where $\tau=t / t_{a}$. Inspection of Figure 12 shows that the data does indeed collapse to Equation 9 by using the $\tau$ scaling.

\section{Scaling Methods for Analyzing the Dusty Flow}

To begin, one must define a coordinate system for sampling the numerical solution. Since the problem is not self-similar, there are no preferred coordinates. We chose two systems:

$$
\chi_{i}=\left\{\begin{array}{l}
\text { fixed ground ranges } \\
r / R_{s}=\text { constant }
\end{array}\right.
$$

The first corresponds to the fixed coordinates used for field-test measurements; the second represents blast wave coordinates that have been used in previous studies (Kuhl et al. 1992). The flow field was then sampled every time step at $\chi_{i}=$ constant in every fine-grid cell and stored as a state vector $\phi\left(\chi_{i}, z, t\right)$ for later statistical analysis (where $\phi$ denotes all relevant flow field variables).

Next, the mean visual dusty boundary layer height $\bar{z}_{v}\left(\chi_{i}, t\right)$, i.e., the height where the dust concentration approaches zero, was measured. Also, the mean fluidized bed height $\bar{z}_{\mathrm{FB}}\left(\chi_{i}, t\right)$ was recorded. This allows one to define a dusty-boundary-layer scaled height $y$ :

$$
y=\left\{\begin{array}{cc}
{\left[z-\bar{z}_{\mathrm{FB}}\left(\chi_{i}, t\right)\right] /\left[\bar{z}_{v}\left(\chi_{i}, t\right)-\bar{z}_{\mathrm{FB}}\left(\chi_{i}, t\right)\right]} & z>z_{\mathrm{FB}} \\
{\left[z-\bar{z}_{\mathrm{FB}}\left(\chi_{i}, t\right)\right] / \bar{z}_{\mathrm{FB}}\left(\chi_{i}, t\right)} & z \leq z_{\mathrm{FB}}
\end{array}\right.
$$

Then the solution was interpolated at constant $y$ values to produce the state vector $\phi\left(\chi_{i}, y, t\right)$.

Next, the freestream conditions were defined as the flow field at 1.5 times the dust layer height, i.e., $y_{\infty} \equiv 1.5 y$. This establishes the local, instantaneous freestream conditions:

$$
\phi_{\infty}\left(\chi_{i}, t\right) \equiv \phi\left(\chi_{i}, y_{\infty}, t\right)
$$




\section{Problem 3. Blast Wave Reflections from Ground Surfaces}

that will be used to nondimensionalize the solution in the mixing layer. In this way, one can define " $y$-scaled waveforms"

$$
\Phi\left(\chi_{i}, y, t\right)=\phi\left(\chi_{i}, y, t\right) / \phi_{\infty}\left(\chi_{i}, t\right)
$$

which will be used in the statistical analysis. Figure 13 presents an example of such nondimensional histories of the radial velocity at $\chi=600 \mathrm{~m}$ and $y=0.8$ and 0.9 .

Point values of a turbulent flow are not particularly useful for fluid-dynamic interpretations; some form of averaging is always required. Here we have time-averaged the nondimensional waveforms to evaluate the mean-flow profiles:

$$
\bar{\phi} / \phi_{\infty}=\bar{\Phi}\left(\chi_{i}, y\right)=\int_{t_{1}}^{t_{2}} \Phi\left(\chi_{i}, y, t\right) d t /\left(t_{2}-t_{1}\right)
$$

For example, averaging the velocity histories in Figure 13 over the precursor duration gives a value of $\bar{u} / u_{\infty}=2.7$ at $y=0.8$ and a value $\bar{u} / u_{\infty}=2.38$ at $y=0.9$. Given the mean values $\bar{\Phi}$, one can then calculate the root-mean-squared (r.m.s.) fluctuations:

$$
\overline{\left[\Phi^{\prime}\left(\chi_{i}, y\right)\right]^{2}}=\int_{t_{1}}^{t_{2}}\left[\Phi\left(\chi_{i}, y, t\right)-\bar{\Phi}\left(\chi_{i}, y\right)\right]^{2} d t /\left(t_{2}-t_{1}\right)
$$

and

$$
\phi^{\prime} / \phi_{\infty}=\sqrt{\overline{\left[\Phi^{\prime}\left(\chi_{i}, y\right)\right]^{2}}}
$$

This analysis then allows one to construct the profiles in the mixing layer that are described in the next section.

\section{Wall-Jet Profiles}

\subsection{Freestream-Scaled Profiles}

The freestream-scaled mean flow profiles in the precursor wall jet are presented parametrically as a function of range in Figure 14. The profiles are quite smooth, indicating that the duration of the time averaging was adequate. The velocity profiles exhibit the characteristic shape of a wall jet. The velocity is zero in the fluidized bed $(y<0)$, it rises to a peak of two to three times $u_{\infty}$, and then decays to the freestream value above the jet $(y>1)$.

The density profiles start at a value of about $1000 \rho_{\infty}$ in the soil bed, drop to a plateau of about $40 \rho_{\infty}$ in the fluidized bed, and then decay to a value of one in the freestream. The profiles seem to converge to a point $\bar{\rho} / \rho_{\infty}=10$ near $y=0$. As in previous studies (Kuhl et al. 1990), leads to an operational definition of the mean height of the fluidized bed, namely, $y_{o}(\chi)$ is defined as the $y$ where $\bar{\rho} / \rho_{\infty}=10$. Typically, this is within one computational cell of $y=o$. This $y_{0}$ will be used later to scale the boundary layer profiles. 


\section{Problem 3. Blast Wave Reflections from Ground Surfaces}

The mean pressure profiles are relatively constant across the layer. The dynamic pressure profiles reach a peak value of 6 to 8 times $q_{\infty}$, which is characteristic of precursor wall jets. Increases in the pitot stagnation pressure are even more dramatic.

The freestream-scaled fluctuation profiles of the precursor wall jet are presented in Figure 15. The profiles are smooth and well ordered, again indicating that the time averaging was adequate to produce reasonable second moments. Streamwise velocity fluctuations reach a peak value of $u^{\prime} / u_{\infty} \simeq 1$ in the jet, and are approximately independent of range. Static pressure fluctuations range from ten to twenty percent of $p_{\infty}$. However, dynamic pressure fluctuations are large - typically 200 to 400 percent of $q_{\infty}$. Similarly, stagnation pressure fluctuations are very large - five to ten times $p_{0 \infty}$ - as is typical of turbulent wall jets.

\subsection{Wall-Jet-Scaled Profiles}

In order to facilitate the comparison with other wall jets, the profiles were replotted using wall jet scaling:

$$
\eta_{J}=\left(y-y_{o}\right) /\left(y_{*}-y_{o}\right)
$$

where

$$
\begin{aligned}
y_{o} & \left.=\text { mean height of the fluidized bed(i.e., } \bar{\rho} / \rho_{\infty}=10\right) \\
y_{*} & =y \text { where } \bar{u}=u_{*} \\
u_{*} & =\left(u_{m}+u_{\infty}\right) / 2 \\
u_{m} & \left.=\text { maximum value of } \bar{u} \text { in the jet (at } y=y_{m}\right)
\end{aligned}
$$

In addition, the velocities were nondimensionalized with $u_{m}$, while the densities and pressures were nondimensionalized with their values at $y=y_{m}$ (e.g., $\rho_{m}=\rho\left(y_{m}\right), p_{m}=p\left(y_{m}\right)$, etc.).

The resulting mean-flow profiles are presented in Figure 16. This wall-jet scaling procedure works extremely well. It demonstrates that the profiles are indeed similar and essentially independent of range (except for the $900 \mathrm{-m}$ station where the wall jet had dissipated, and two separate shear layers remained). This is especially true for $\bar{u}, \bar{\rho}, \bar{q}$, and $\bar{p}_{o}$. Also notice that by construction, the density profiles converge to a value of $\bar{\rho} / \rho_{m}=10$ at $\eta_{J}=0$.

The r.m.s. fluctuation profiles are presented in Figure 17. The streamwise velocity fluctuations reach a peak value of about $u^{\prime} / u_{m}=0.5$, and are self-similar for ranges of 400 , 500 , and $600 \mathrm{~m}$. At $700 \mathrm{~m}$ a transition occurs, and at 800 and $900 \mathrm{~m}$ there are two distinct peaks associated with the separate shear layers (one on top of the thermal layer and the other associated with the dusty boundary layer) associated with the precursor cleanup phase. Vertical velocity fluctuations range from 10 to 20 percent of $u_{m}$ until the ranges of 800 and $900 \mathrm{~m}$, where the wall jet fluid upwells to form the dust pedestal. Density fluctuations reach a peak value of $10 \rho_{m}$ at the bottom of the boundary layer. Static pressure fluctuations are typically 10 to 20 percent. 


\section{Problem 3. Blast Wave Reflections from Ground Surfaces}

\subsection{Boundary-Layer-Scaled Profles}

In order to facilitate comparisons with other dusty boundary layers, the profiles were replotted using boundary layer scaling:

$$
\eta_{\mathrm{BL}}=\left(y-y_{0}\right) /\left(y_{\mathrm{BL}}-y_{o}\right)
$$

and $y_{\mathrm{BL}}$ is defined as the $y$ where $\bar{u}=0.99 u_{m}$. This implies that the nondimensional boundary layer height in the wall jet is

$$
\delta / z_{v}=y_{\mathrm{BL}}-y_{0}
$$

The mean-flow profiles are presented in Figure 18. With the exception of stations $800 \mathrm{~m}$ and $900 \mathrm{~m}$, the streamwise velocity profiles are essentially similar and independent of range. Dynamic pressure and stagnation pressure profiles show big losses near the bottom of the boundary layer - due to entrainment of zero-momentum dust from the fluidized bed.

The r.m.s. fluctuation profiles of the wall jet boundary layer are presented in Figure 19. Most notably, the Reynolds stress is zero in the fluidized bed $\left(\eta_{\mathrm{BL}}<0\right)$.

The profiles of the mean mass and momentum flux are depicted in Figure 20. Most significantly, the streamwise mass flux is approximately constant $\left(\overline{\rho u} \simeq \rho_{m} u_{m}\right)$ in the boundary layer, while the mean vertical mass flux is essentially zero.

The calculated boundary layer profiles in the wall jet have been replotted in semilog coordinates in Figure 21. Close examination shows that profiles from the precursor wall jet are very similar to our previous simulations of a dusty boundary layer behind a normal shock and are in good agreement with the Laser Dopler Velocimetry (LDV) measurements (shaded region; Batt el al. 1988) performed in the TRW shock tube of the latter case. Similar comments apply to the calculated mixture specific volume profiles, which agree with the streak x-ray measurements (Batt et al. 1988). In lieu of any dusty wall jet data, we suggest

that this agreement with shock tube data provides the best fundamental validation of the accuracy of convective mixing simulations of the dusty precursor flow.

\section{Comparison with Self-similar Wall Jets}

In this section we compare the profiles of the present blast wave precursor with our previous numerical simulations of self-similar wall jets, namely: (1) A dusty precursor wall jet generated by a normal shock interacting with a helium layer (Kuhl et al. 1989); (2) A dusty wall jet embedded in a double-Mach-reflection DMR flow (Kuhl et al. 1991); and (3) A clean wall jet formed in a steady windtunnel flow (Kuhl et al. 1990).

Comparisons of the streamwise velocity profiles are presented in Figure 22. The present solution is shown in Figure 22a. The wall jet scaling $\eta_{J}$ seems to collapse the profiles to essentially a single curve, except at the farthest ranges of $800 \mathrm{~m}$ and $900 \mathrm{~m}$ where the free 


\section{Problem 3. Blast Wave Reflections from Ground Surfaces}

shear layer and the wall boundary layer do not strongly interact. The profiles for the squarewave dusty precursor calculation are presented in Figure 22b. There, the parameter $\xi$ denotes the nondimensional distance along the jet $(\xi=0$ represents the start of the jet and $\xi=1$ denotes the tip of the jet). Again, the wall jet scaling collapses the profiles, except near $\xi=1$ where the two shear layers do not interact. The profiles for the DMR dusty wall jet are shown in Figure 22c. There, the parameter $\xi$ denotes the nondimensional distance along the jet $(\xi=0$ represents the start of the jet and $\xi=1$ denotes the foot of the Mach stem). Again, the wall jet scaling collapses the profiles, except at $\xi=0.7$ and 0.8 where the two shear layers do not interact. The profiles for the steady, clean wall jet are shown in Figure 22d. At $x=85$, one finds a classic wall jet profile shape; however, by $x=170$ the flow has separated from the wall, due to large-scale vortex pairings between the free shear layer and wall boundary layer. In summary, the profiles of the present calculations are quite similar to other wall jet flows; however, the velocity ratio in the freestream $\left(\eta_{J} \geq 2\right)$ is problem-dependent.

The corresponding streamwise-velocity-fluctuation profiles are compared in Figure 23. The wall jet scaling collapses the profiles for the present calculation (Fig. 23a), except for ranges $800 \mathrm{~m}$ and $900 \mathrm{~m}$ where the wall jet has mostly dissipated. Peak values are $u^{\prime} / u_{m} \simeq$ 0.45. Two distinct peaks are evident in the square-wave dusty precursor case (Fig. 23b). Evidently, the two shear layers do not interact so strongly in this case; the peak value is $u^{\prime} / u_{m} \simeq 0.35$. The DMR dusty wall jet (Fig. 23c) also has double-peaked profiles, with the maximum value of $u^{\prime} / u_{m} \simeq 0.28$ near the wall. The steady, clean wall jet also has a doublepeaked profiles, with a maximum value of $u^{\prime} / u_{J}=0.28$. In summary, peak fluctuations are similar in all cases (ranging from 30 to 40 percent of $u_{m}$ ). However, the present blast wave case has basically single-peak profiles, indicating strong interactions between the two embedded shear layers; the other cases have double-peaked profiles that are indicative of two distinct shear layer regions.

The corresponding transverse-velocity-fluctuation profiles are compared in Figure 24. In the present blast wave case (Fig. 24a), the profiles reach a peak value of about $v^{\prime} / u_{m}=0.2$, with the exception of stations $800 \mathrm{~m}$ and $900 \mathrm{~m}$, where the upwelling of the flow is forming the dust pedestal. The other cases exhibit double-peak profiles - indicative of two distinct shear layer regions. Maximum fluctuations range from $v^{\prime} / u_{m}=0.08$ to $v^{\prime} / u_{J}=0.32$, depending on the case considered.

The corresponding semi-log boundary layer profiles are compared in Figure 25. The calculated profiles for the present blast wave case (Fig. 25a) are in excellent agreement with the LDV measurements of dusty boundary layer profiles in the TRW shock tube tests (Batt et al. 1988) and also with our previous simulations labeled NS (Kuhl et al. 1990) of those tests. They are also similar to the profiles of our previous square-wave precursor flow (Fig. 25b), and DMR wall jet (Fig. 25c), where dust density effects were strong. These comparisons then suggest that the present calculations of a blast wave precursor produce a wall jet similar to those found in self-similar problems that have been studied previously (e.g., Bajura and Catalano 1975; Wygnanski et al. 1992). 


\section{Problem 3. Blast Wave Reflections from Ground Surfaces}

\section{Summary and Conclusions}

Careful examination of the flow visualization (e.g., Figures 3 to 10) revealed a wealth of information on the fluid dynamics of turbulent dusty flows in explosions. These calculations showed that there are three distinct regions of dusty flow: (1) a heave region near ground zero; (2) a boundary layer region; and (3) a wall jet. The precursor formed well within the regular reflection regime $(G R \simeq 130 \mathrm{~m})$, and the Mach stem formation was delayed until $G R \simeq 350 \mathrm{~m}$ (i.e., well past the Mach transition, $G R \simeq 200 \mathrm{~m}$ ), because of the precursor shock structure.

The dust heave region $(0<G R<300 \mathrm{~m})$ was created by a sound speed effect whereby pressure gradients relaxed faster in the air than in the dust layer; this pressure difference heaved some of the dust upward. Initially the layer grew by Richtmyer-Meshkov fingering mechanisms, but eventually it grew by Kelvin-Helmholtz mixing, and formed the leading edge of the dust stem.

A dusty boundary layer was left behind in the wake of the wall jet. During the positive phase, it grew as a power-law function of time: $\delta_{v}=1.4 \tau^{1.5}\left(\mathrm{~m} / \mathrm{KT}^{1 / 3}\right)$, due to entrainment and merging processes. During the negative phase, opposite sign vorticity was created by blast wave pressure gradients. Dust scouring occurred naturally via entrainment of dust from the fluidized bed due to the velocity field of the rotational structures of the boundary layer.

A turbulent wall jet was induced by the precursor shock structure. The jet formed at a range $G R \simeq 240 \mathrm{~m}$, which is outside the classical regular reflection region $(G R<200 \mathrm{~m})$ of the blast wave. In the region $240 \mathrm{~m}<G R<600 \mathrm{~m}$, the wall jet was strongly coupled to the precursor shock structure, and this enforced a predominantly two-dimensional character to the flow. Strong interactions were observed between the free shear layer and the boundary layer, leading to pairings and large-scale mergings that mixed the dust throughout the wall jet. Such processes also caused local separations and intermittent dust scouring.

Eventually the wall jet fell behind the precursor, due to the inertia of the dusty flow. When the wall jet decoupled from the precursor shock structure $(600 \mathrm{~m}<G R<800 \mathrm{~m})$, it became unconstrained and quickly broke up into large-scale mixing structures that separated from the ground. The details of the breakup were dependent on the grid resolution, and were fundamentally a three-dimensional phenomenon. Eventually, the wall jet fluid (i.e., the vorticity, dust and thermal layer gas) merged and ballooned upward to form a dust pedestal at $G R \simeq 800 \mathrm{~m}$. Thus, the run length of the wall jet was about $560 \mathrm{~m}$ or $165 \mathrm{H}$ (where $H$ is the thermal layer thickness).

Scaling methods were defined to analyze the dusty flow. The visual dust height $z_{v}(r, t)$ was used to scale the upper portion of the mixing layer, while the fluidized bed height $z_{\mathrm{FB}}(r, t)$ was used to scale the lower portion of the boundary layer. In addition, the flowfield was nondimensionalized by the local, instantaneous freestream conditions $\phi_{\infty}(r, t)$. Timeaveraging of such nondimensional waveforms was adequate to produce smooth, well-behaved mean and r.m.s. profiles. 


\section{Problem 3. Blast Wave Reflections from Ground Surfaces}

It was demonstrated that the wall jet profiles scale with $\left.\eta_{J}=\left(y-y_{0}\right) / y_{*}-y_{0}\right)$ and that the profiles were similar to other wall jet flows. The boundary layer portion of the wall jet scaled with $\left.\eta_{\mathrm{BL}}=\left(y-y_{0}\right) / y_{\mathrm{BL}}-y_{0}\right)$; these profiles were similar to the measured profiles for a dusty boundary layer behind a shock.

Such agreement with data suggests that the two-dimensional flow approximation used here is adequate to capture the mean-flow effects in the mixing layer. However, one might expect that the calculated fluctuations are only qualitatively similar to actual turbulent fluctuations - because of the two-dimensional flow approximation.

We certainly realize that boundary layers are inherently three-dimensional; consequently, we have begun some calculations with our 3D-AMR code. Figure 26 presents an oblique view of the calculated dust concentration surface at $t=1 \mathrm{~s}$. Three-dimensional effects are evident, especially in the boundary layer flow behind the wall jet. Such a three-dimensional approach should be used in future simulations of turbulent mixing layers.

Finally, it is worth noting that these dusty flows are really baroclinic boundary layers, where density replaces the role of viscosity. Large densities in the fluidized bed enforce the condition $\mathbf{u}=0$ at the bottom of the dusty boundary layer by inertia, just as viscosity and the no-slip condition on the wall create clean boundary layers.

\section{Epilogue}

The present approach avoids the perils and limitations of turbulence models that are, by necessity, based on integration of mean-field equations. As Chorin (1975) has pointed out, the laws of fluid dynamics are nonlinear, so the integration of the averaged equations does not equal the average of the integrated equations. The Convective Mixing Model is based on the latter approach, namely, we integrate the equations (by calculating the convective mixing on an adaptive grid) and then average the solution. This approach can provide a more faithful numerical simulation of a wide spectrum of fluid dynamic mixing problems.

\section{Acknowledgements}

This work was sponsored by the Defense Nuclear Agency with a contract to the Lawrence Livermore National Laboratory (IACRO number 92-824, Work Unit 00001), and a contract to the Naval Surface Warfare Center (MIPR-92-588). Part of the work was also performed under the auspices of the U.S. Department of Energy (LLNL contract W-7405-ENG-48). This manuscript was prepared by the staff of Logicon-RDA, under DNA contract number DNA001-88-C-0046. Their support is gratefully acknowledged. The invitation of the Institute of Fluid Science, and the hospitality of Professor K. Takayama (Director of the Shock Wave Research Center) was greatly appreciated. 


\section{Problem 3. Blast Wave Reflections from Ground Surfaces}

\section{References}

Bajura, R. A. and Catalano, M. R., "Transition in a two-dimensional plane wall jet," J. Fluid Mech., 70, 1975, pp. 773-799.

Barthel, J., "2-D Hydrocode computations csing a $\kappa-\epsilon$ turbulence model: model description and test calculations," SSS-R-85-7115, S-Cubed, La Jolla, California, 1985.

Batt, R. G., Kulkarny, V. A., Behrens, H. W., and Rungaldier, H., "Shock-induced boundary layer dust lofting," Shock Tubes and Waves, edited by H. Grönig, VCH, Weinheim Germany, 1988, pp. 209-215.

Berger, M. J. and Colella, P. "Local adaptive mesh refinement for shock hydrodynamics," J. Comput. Phys., 82, 1989, pp. 64-84.

Carpenter, H. J., Engler, M. J. and McCaffree, L. A., Pre-shock thermal layer soundspeeds developed from nuclear test data, DNA-TR-89-190, Defense Nuclear Agency, Wash., D.C., 1991.

Chorin, A. J., Lectures on Turbulence Theory, Publish or Perish, Inc., Boston, 1975 (vid. esp. Chapter I-3, "An example of the perils of dishonesty," pp. 21-23).

Colella, P., and Glaz, H. M., "Efficient solution algorithms for the Riemann problem for real gases," Journal of Computational Physics, 59, No. 2, 1985, pp. 264-289.

Colella, P., Ferguson, R. E., Glaz, H. M., and Kuhl, A. L., "Mach reflection from an HE-driven blast wave," Dynamics of Explosions: Progress in Astronautics and Aeronautics 106 (ed. J. R. Bowen, J. -C. Leyer, R. I. Soloukhin), AIAA, Wash., D.C., 1986, pp. $388-421$.

Donaldson, C. et al., "Second-order closure model: comparison with a number of complex turbulent flows," ARAP 469, Aeronautical Research Associates of Princeton, Princeton, New Jersey, 1982.

Glaz, H. M., Ferguson, R.E., Collins, J. P., Chien, K.-Y., and Kuhl, A. L., "Godunov models for dusty gas flows," 19th Int. Symp. on Shock waves, ed. R. Brun (in preparation).

Kuhl, A. L., Glowacki, W., Chien, K.-Y., Ferguson, R. E., Collins, P., Glaz, H. M., and Colella, P., "Simulation of a turbulent wall jet in a precursor flow," Proceedings of the Eleventh Int. Symposium on the Military Applications of Blast Simulation, Albuquerque, (ed. A. Mark)., Ballistics Research Laboratory, Aberdeen, MD., 1989, pp. 217-244. 


\section{Problem 3. Blast Wave Reflections from Ground Surfaces}

Kuhl, A. L., Chien, K.-Y., Ferguson, R. E., Collins, J. P., Glaz, H. M. and Colella, P., "Simulation of a turbulent dusty boundary layer behind a shock," Current Topics in Shock Waves, edited by Y. W. Kim, American Institute of Physics Press, New York, 1990, pp. 762-769.

Kuhl, A. L., Chien, K.-Y., Ferguson, R. E., Glaz, H. M. and Colella, P., Inviscid dynamics of unstable shear layers, RDA-TR-161604-006, Logicon RDA, Los Angeles, CA, 1990.

Kuhl, A. L., Ferguson, R. E., Chien, K.-Y., Glowacki, W., Collins, J.P., Glaz, H., and Colella, P., "Turbulent wall jet in a mach reflection flow," Dynamics of Detonations and Explosions: Explosion Phenomena, edited by A. L. Kuhl, J.-C. Leyer, A. A. Borisov, W. A. Siriginano, 134, Progress in Astronautics and Aeronautics, New York, 1991, pp. 201-232.

Kuhl, A. L., "A baroclinic model of turbulent dusty flows," UCRL-JC-111404, Lawrence Livermore National Laboratory, Livermore, CA, 1992.

Kuhl, A. L., Ferguson, R. E., Chien, K.-Y., and Collins, J. P., Turbulent dusty boundary layer in an ANFO surface burst explosion, DNA-TR-92-17, Defense Nuclear Agency, Wash., D.C., 1992.

Kuhl, A. L., Ferguson, R.E., Chien, K.-Y, and Collins, J. P., "Unstable wall layers created by shock reflections," Dynamics of Explosion Phenomena (edited by A. L. Kuhl, J. C. Leyer, A. A. Borisov and W. A. Sirignano), 153, Progress in Astronautics and Aeronautics, Wash., D.C., 1993.

Reichenbach, H., "Laboratory-scale airblast precursor experiments: Vol. II - Parametric studies," DNA-TR-85-352-V2, Defense Nuclear Agency, Wash., D.C., 1985.

Reichenbach, H., Scheklinski-Glück, G., and Kuhl, A. L., "Comparison of HOB curves for 0.5-g NP charges with field test data and calculations," DNA-TR-90-223, Defense Nuclear Agency, Wash., D.C., 1992.

Traci, R. M. and Su, F. Y., "Turbulent, two-phase flow modeling for dusty boundary layer analyses," FP1 R88-06-05, Fluid Physics Ind., Encinitas, California, 1988.

Wygnanski, I., Katz, Y. and Horev, E., "Applicability of scaling laws to the turbulent wall jet," J. Fluid Mech., 234, pp. 669-690, 1992. 


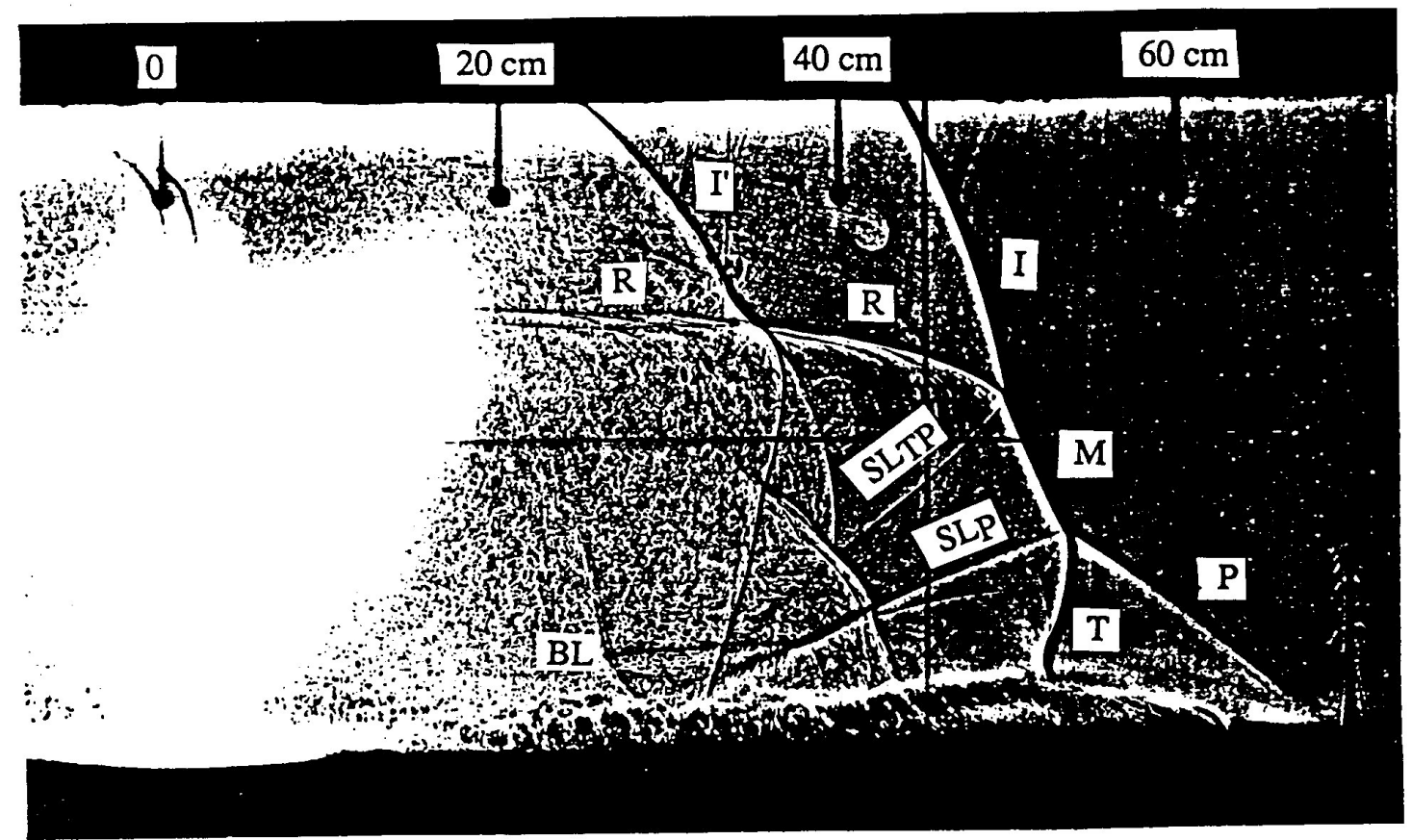

Figure 1. Shadow photograph of a blast wave precursor experiment. (Courtesy of Dr. H. Reichenbach, Emst Mach Institut, Freiburg) 
Problem 3. Blast Wave Reflections from Ground Surfaces

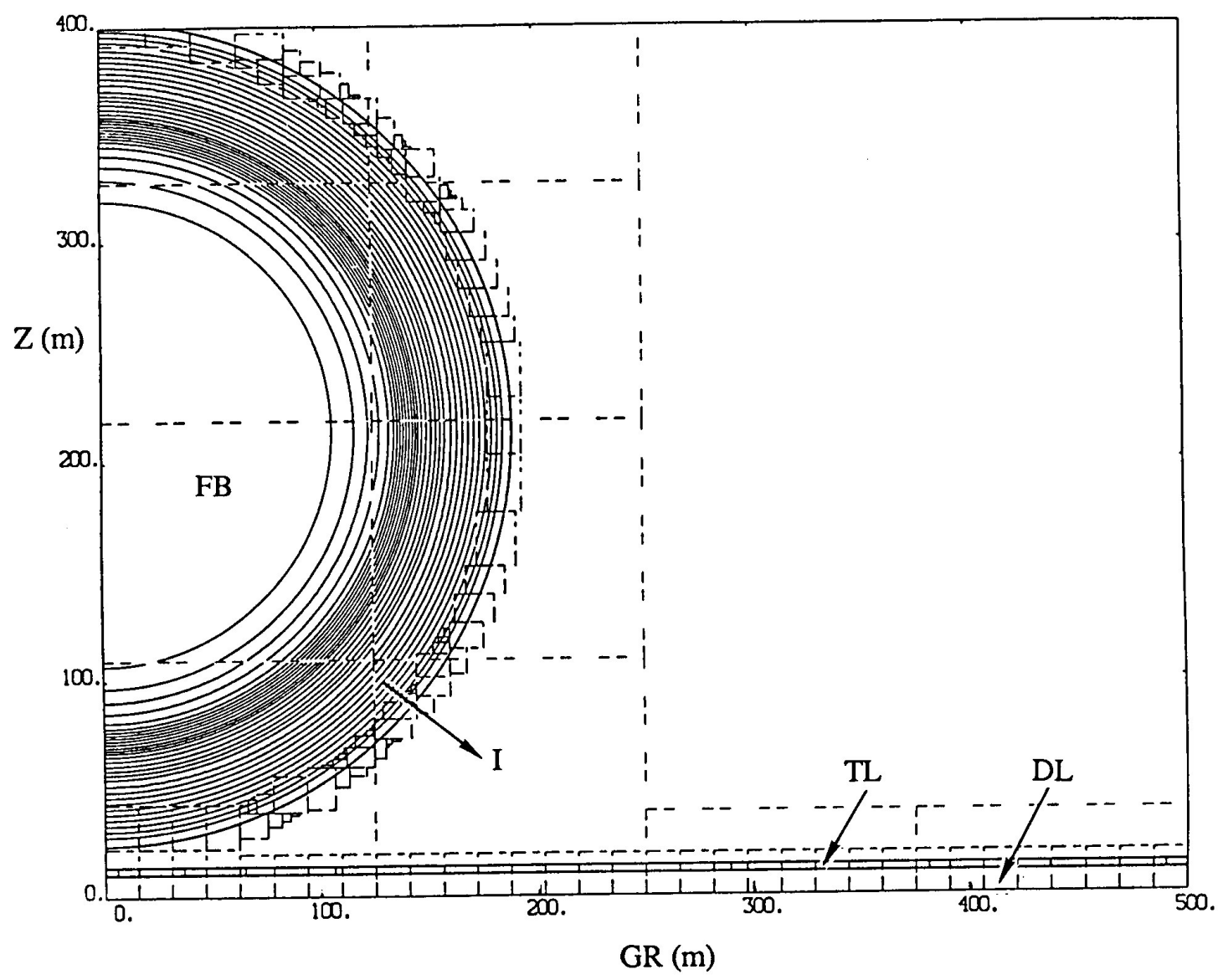

Figure 2. Initial conditions $(t=51.56 \mathrm{~ms})$ of the calculation showing the explosion at $\mathrm{z}=203 \mathrm{~m}$ : incident shock $\mathrm{I}$, fireball $\mathrm{FB}$, thermal layer TL, and dust layer DL ( $\log \rho_{\mathrm{g}}$ contours). 


\section{Problem 3. Blast Wave Reflections from Ground Surfaces}

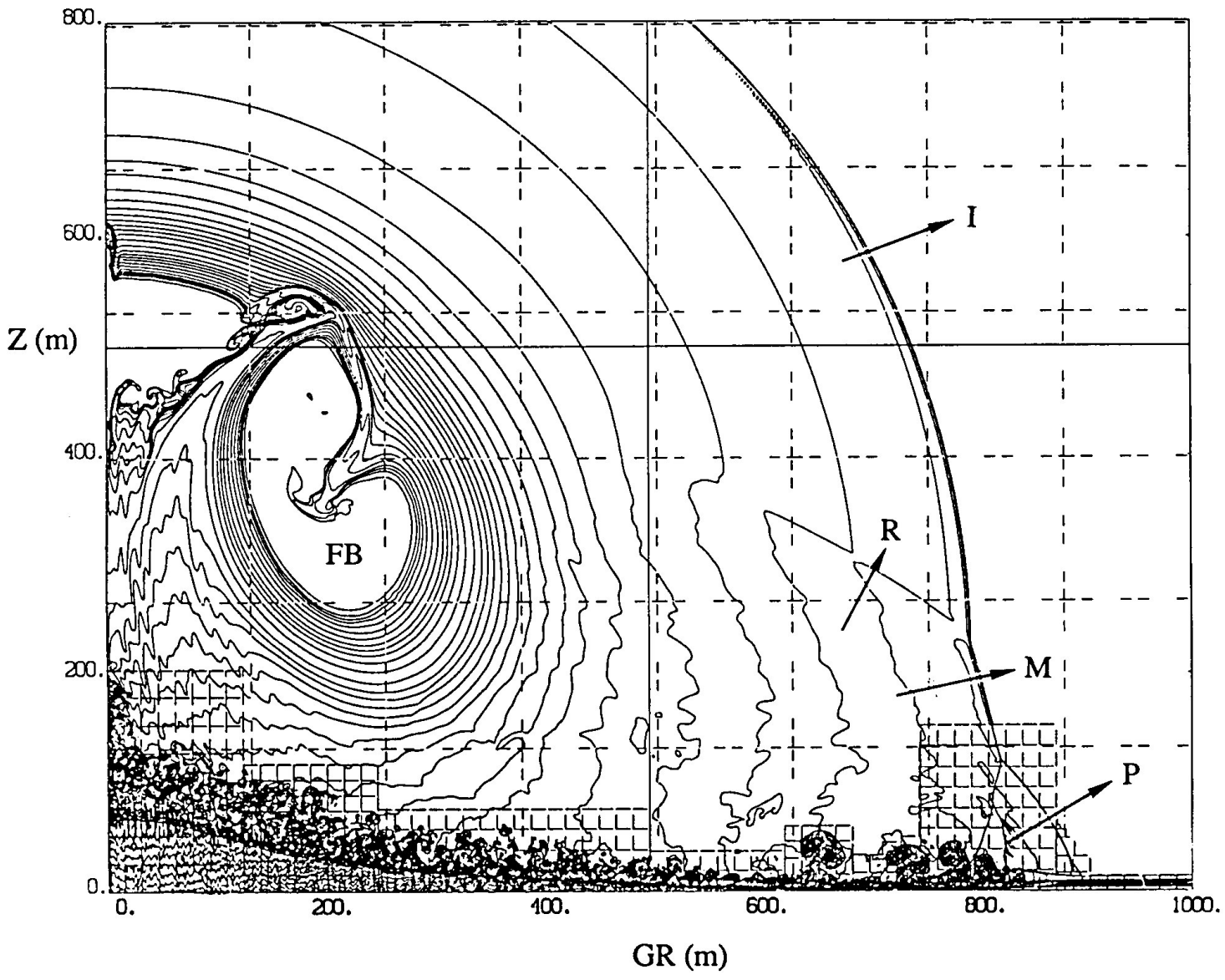

Figure 3. Contours of $\log \left(\rho_{\mathrm{g}}\right)$ showing the overall flowfield at $\mathrm{t}=1027 \mathrm{~ms}$. 


\section{Problem 3. Blast Wave Reflections from Ground Surfaces}
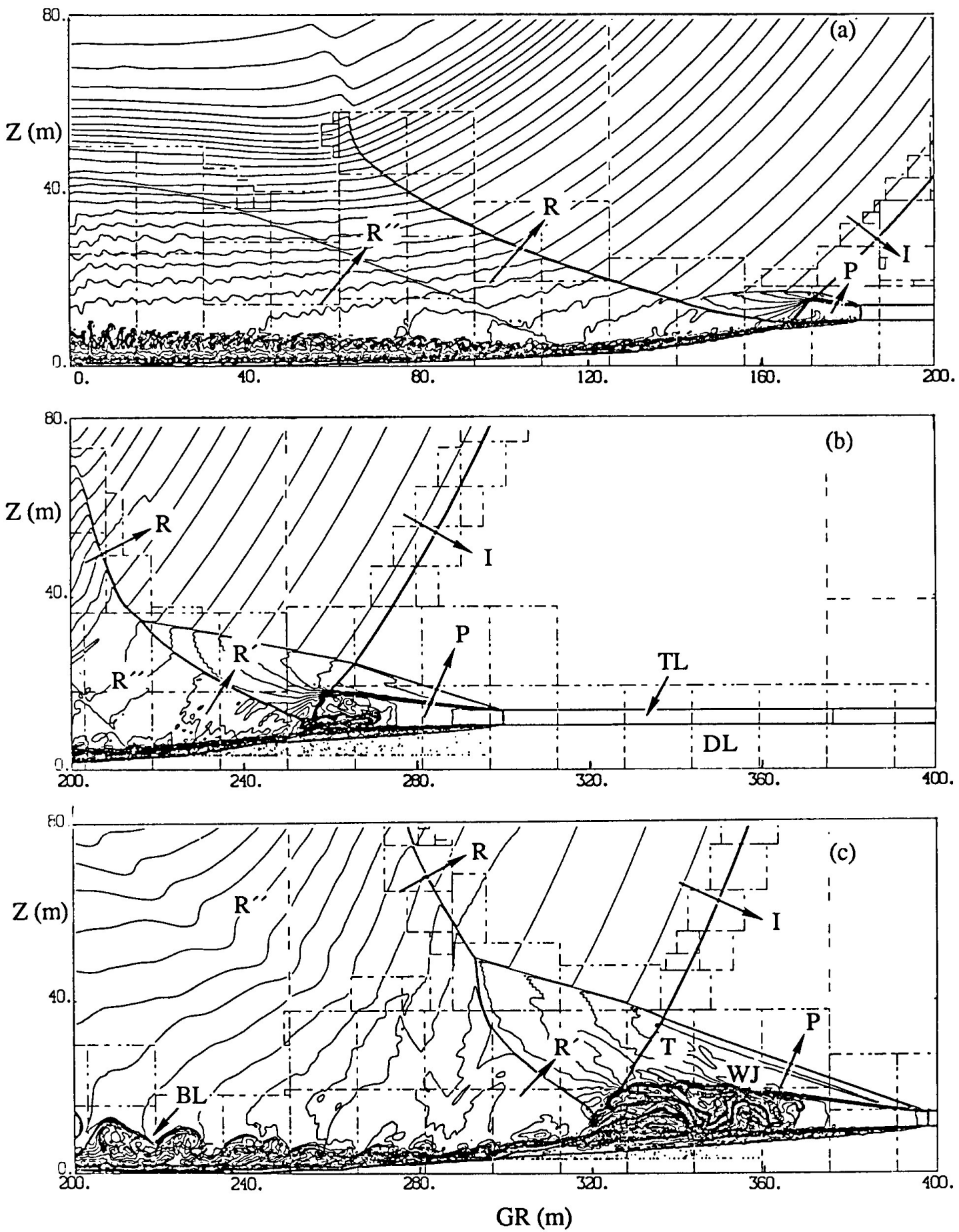

Figure 4 Initiation of the precursor $P$ and the formation of the wall jet:

(a) $\mathrm{t}=109 \mathrm{~ms}$; (b) $\mathrm{t}=176 \mathrm{~ms}$; (c) $\mathrm{t}=248 \mathrm{~ms}\left(\log \rho_{\mathrm{g}}\right.$ contours). 

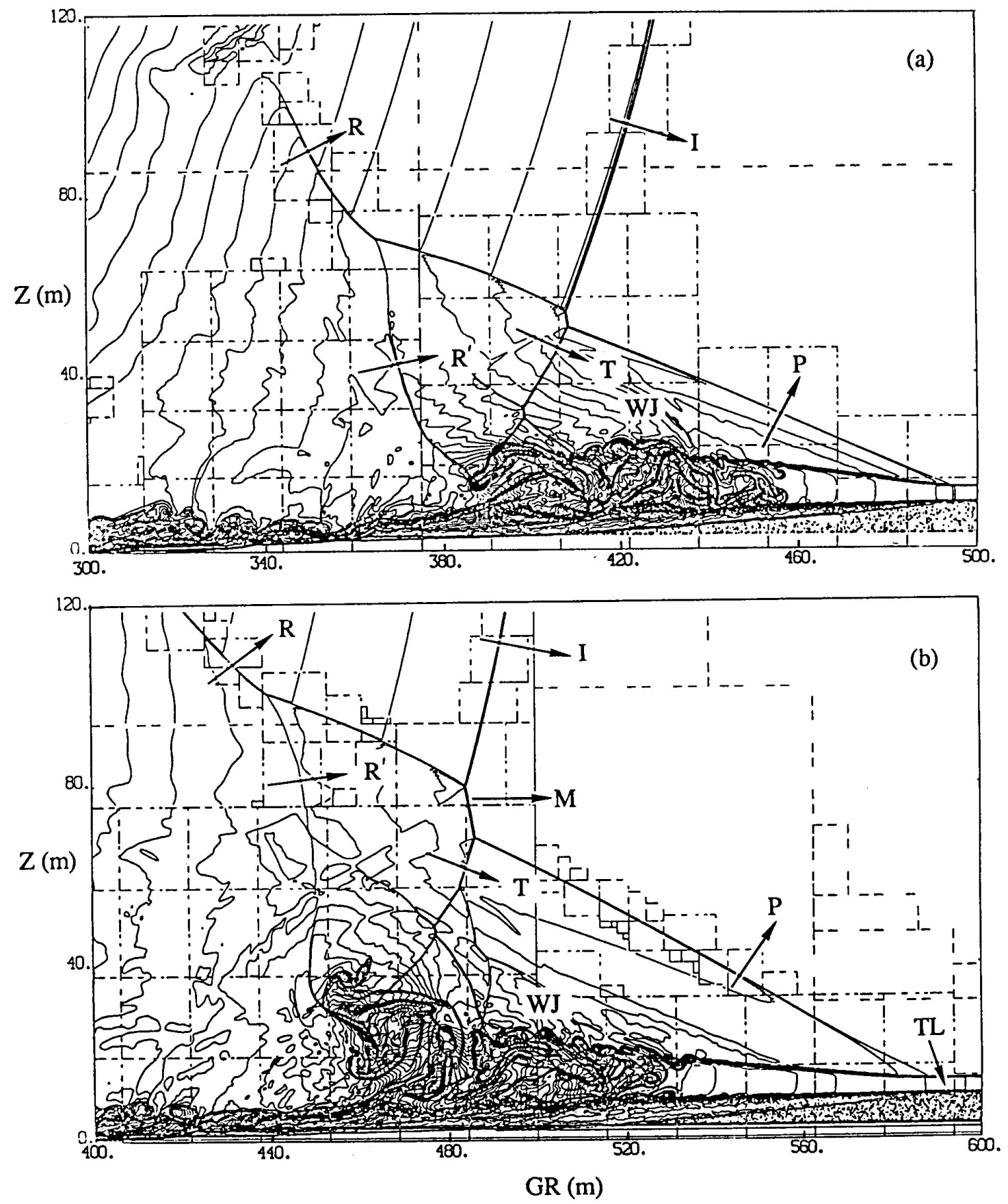

Figure 5. Growth of the precursor $P$ and the development of the wall jet: (a) $\mathrm{t}=332 \mathrm{~ms}$; (b) $\mathrm{t}=438 \mathrm{~ms}\left(\log \rho_{\mathrm{g}}\right.$ contours). 
Problem 3. Blast Wave Reflections from Ground Surfaces
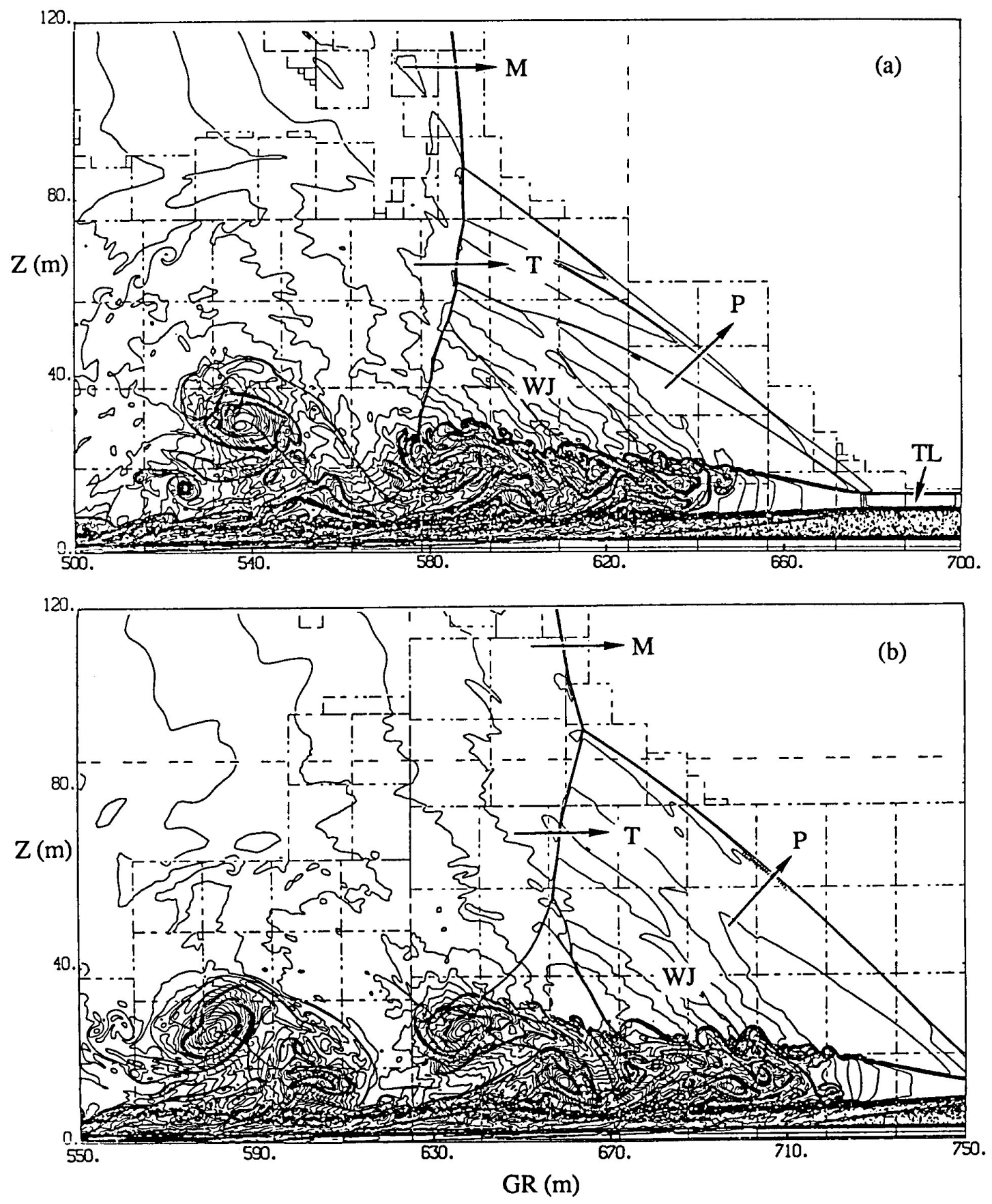

Figure 6. Precursor propagation and transition of the wall jet:

(a) $\mathrm{t}=603 \mathrm{~ms}$; (b) $\mathrm{t}=730 \mathrm{~ms}\left(\log \rho_{\mathrm{g}}\right.$ contours). 


\section{Problem 3. Blast Wave Reflections from Ground Surfaces}
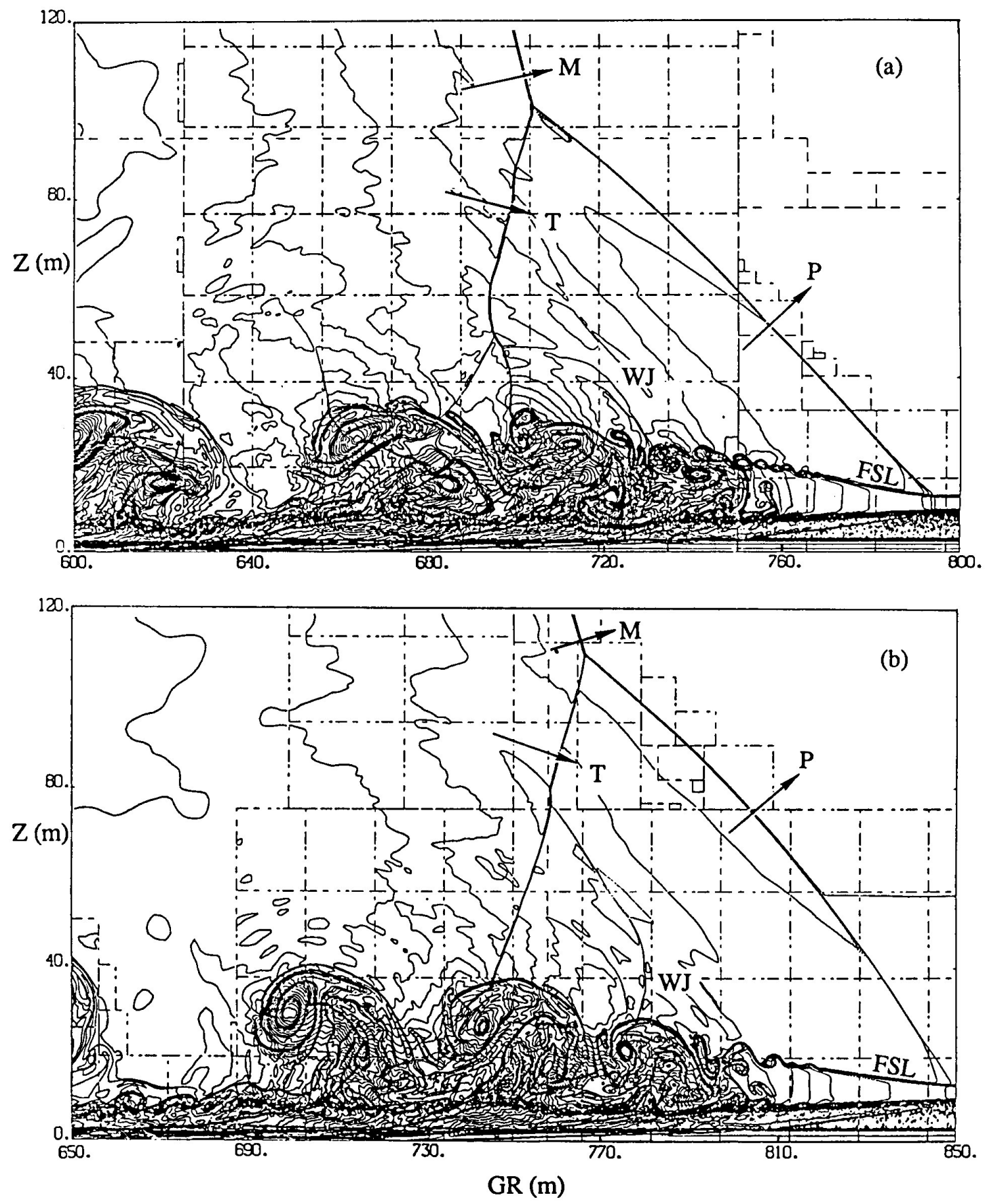

Figure 7. Decay of the precursor $P$ and breakup of the wall jet:

(a) $\mathrm{t}=800 \mathrm{~ms}$; (b) $\mathrm{t}=916 \mathrm{~ms}\left(\log \rho_{\mathrm{g}}\right.$ contours). 
Problem 3. Blast Wave Reflections from Ground Surfaces

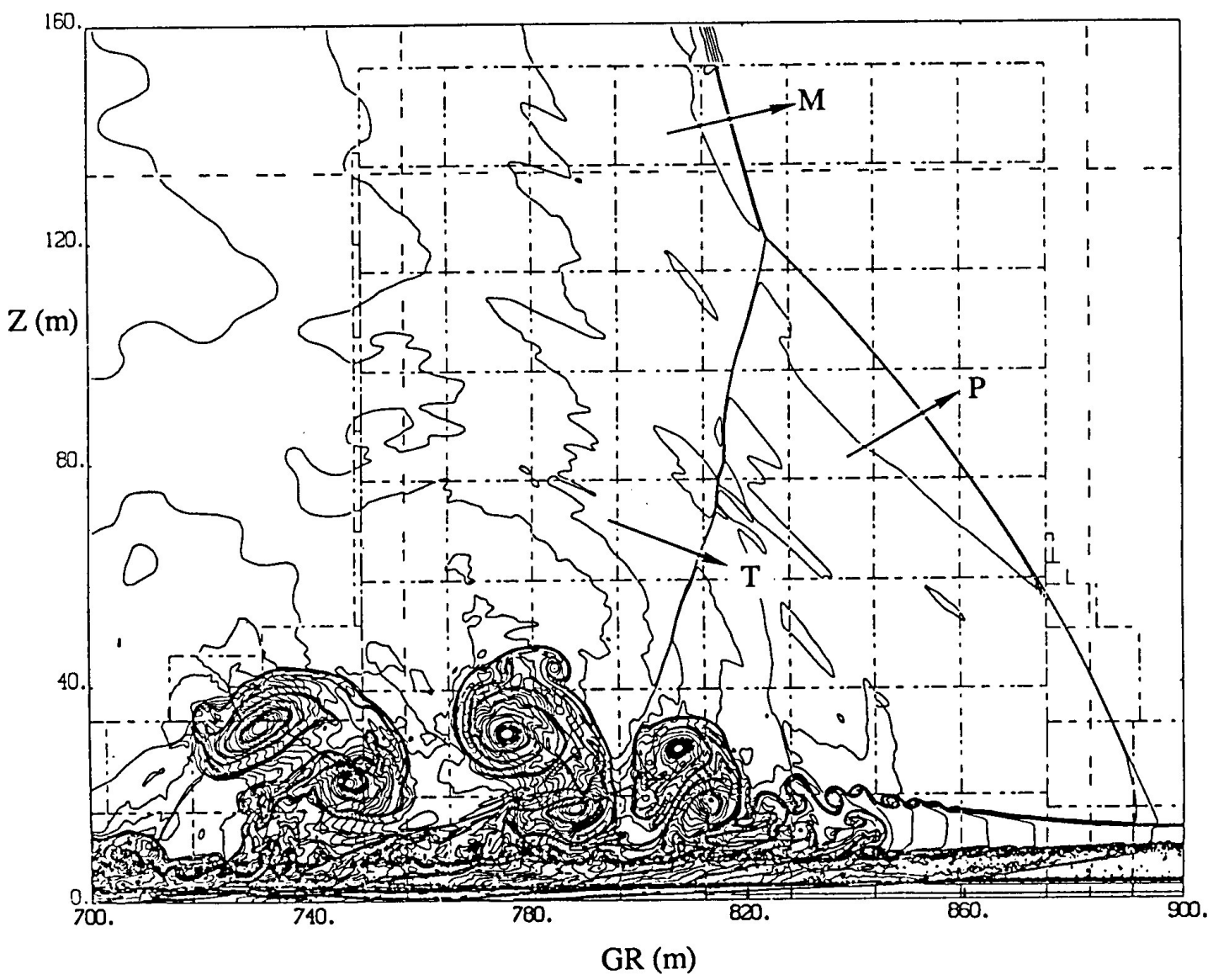

Figure 8. Decoupling of the wall jet from the precursor shock structure and the formation of the dust pedestal at $\mathrm{t}=1027 \mathrm{~ms}$ ( $\log \rho_{\mathrm{g}}$ contours). 
Problem 3. Blast Wave Reflections from Ground Surfaces
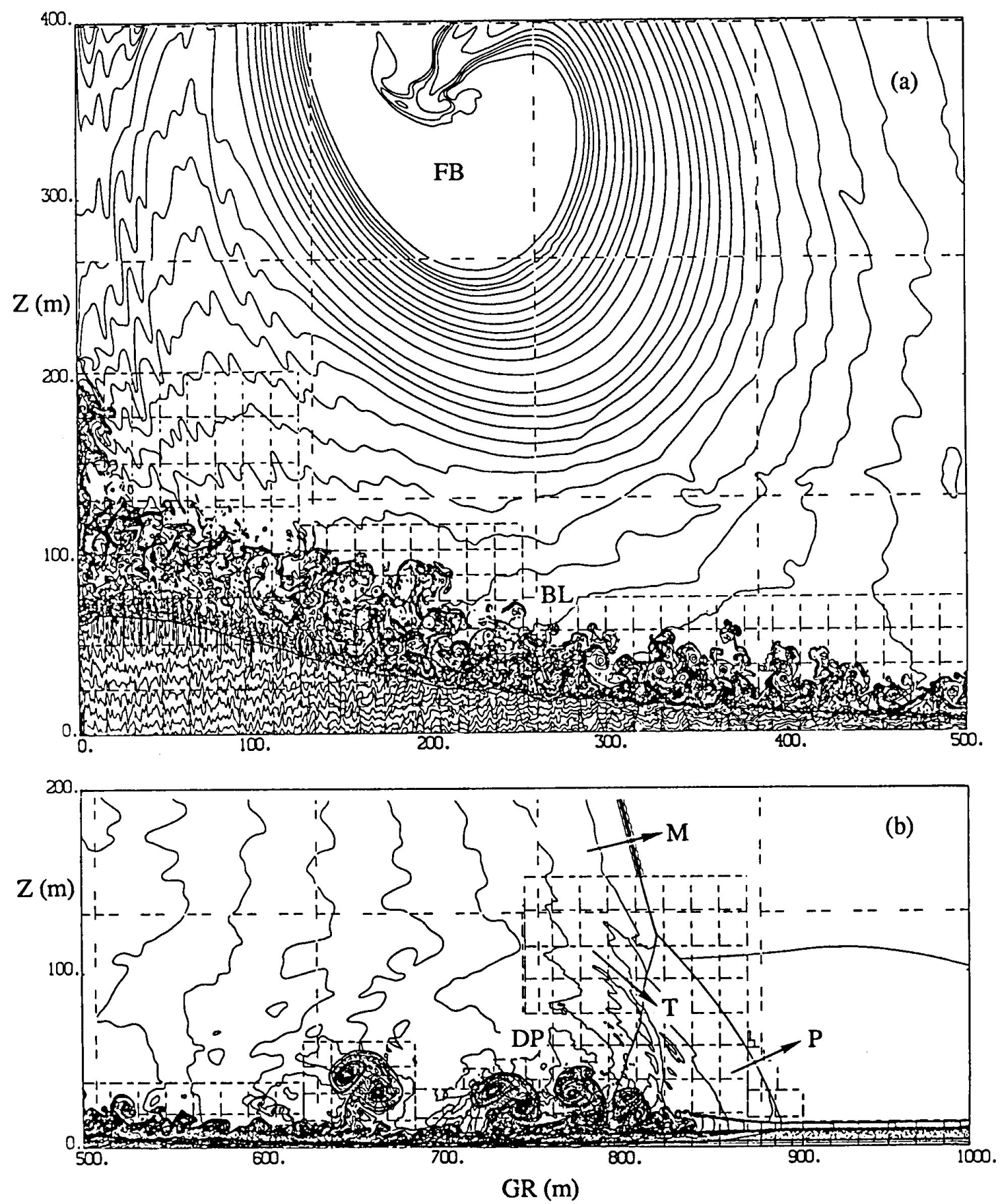

Figure 9. Entrainment of the dusty boundary up the axis at ground zero and the formation of the dust pedestal DP at $G R \approx 800 \mathrm{~m}(\mathrm{t}=1027 \mathrm{~ms}$, $\log \rho_{\mathrm{g}}$ contours). 
Problem 3. Blast Wave Reflections from Ground Surfaces

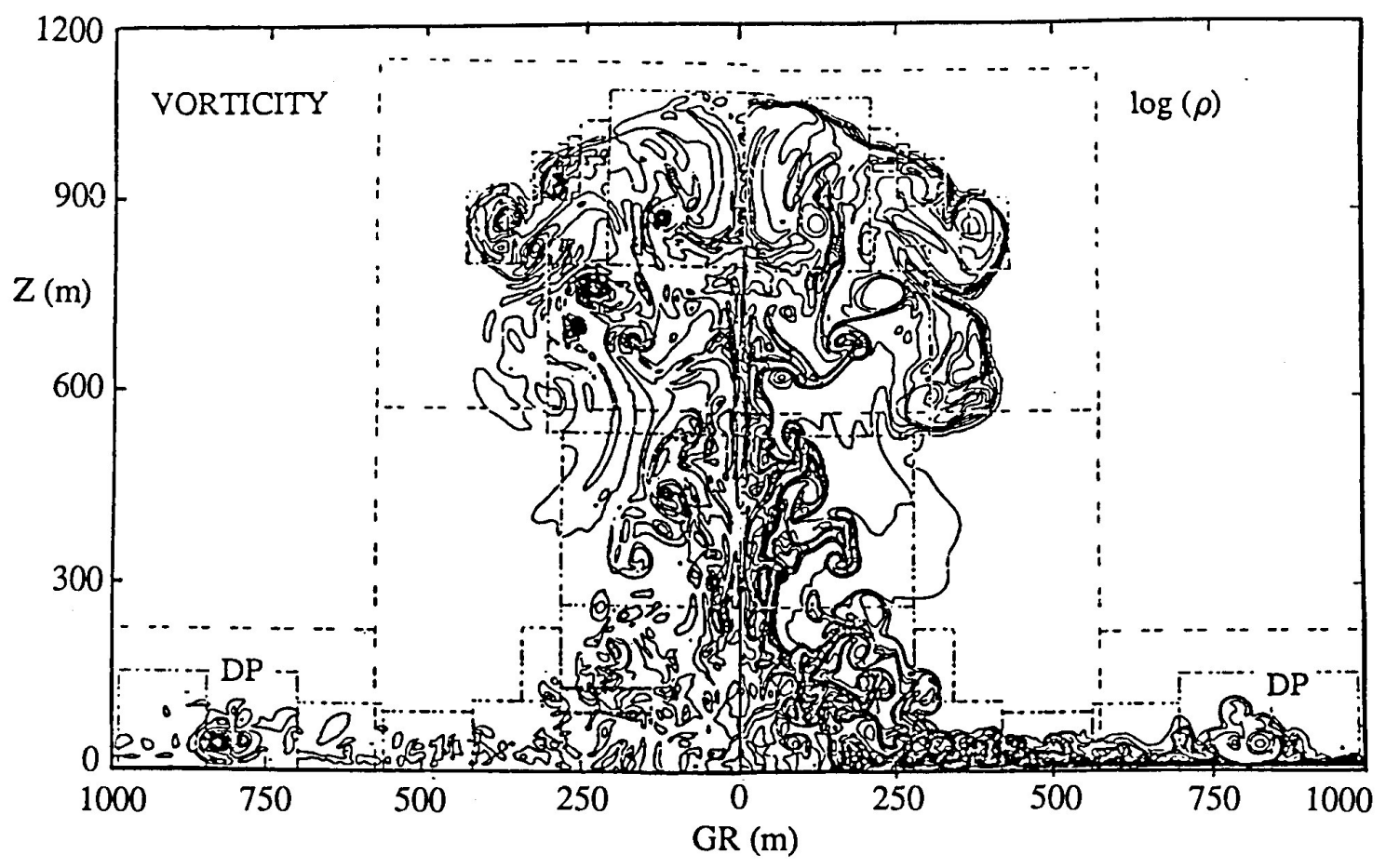

Figure 10. Late-time flowfield showing the entrainment of dust from the boundary layer up the dust stem and into the turbulent cloud; also visible is the dust pedestal $D P$ at $G R \approx 800 \mathrm{~m}: t=9.6 \mathrm{~s}$; left panel shows vorticity contours; right panel shows $\log \rho$ contours. 
Problem 3. Blast Wave Reflections from Ground Surfaces

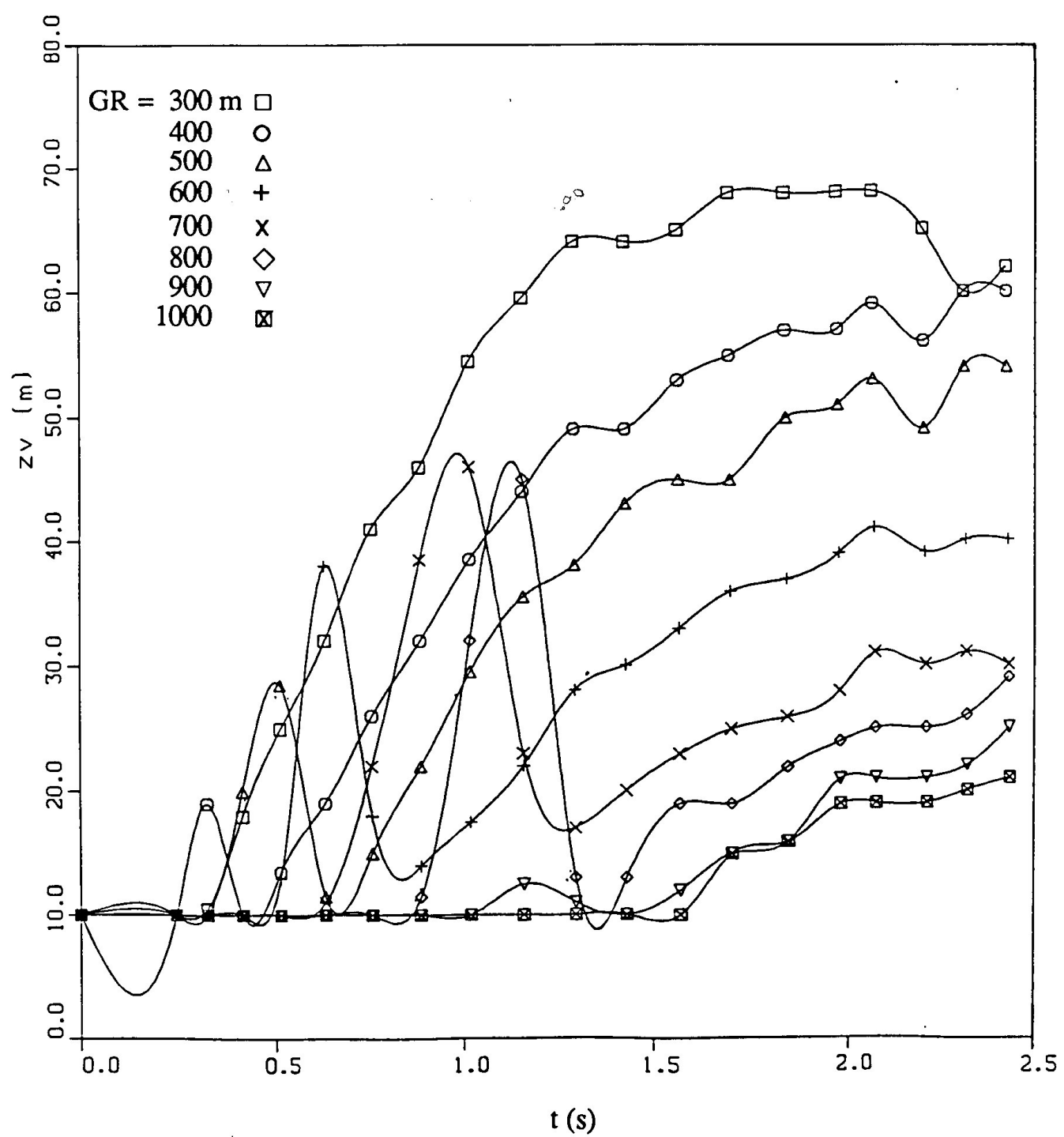

Figure 11. Evolution of the visual dust height $z_{v}$ at various ranges. 
Problem 3. Blast Wave Reflections from Ground Surfaces

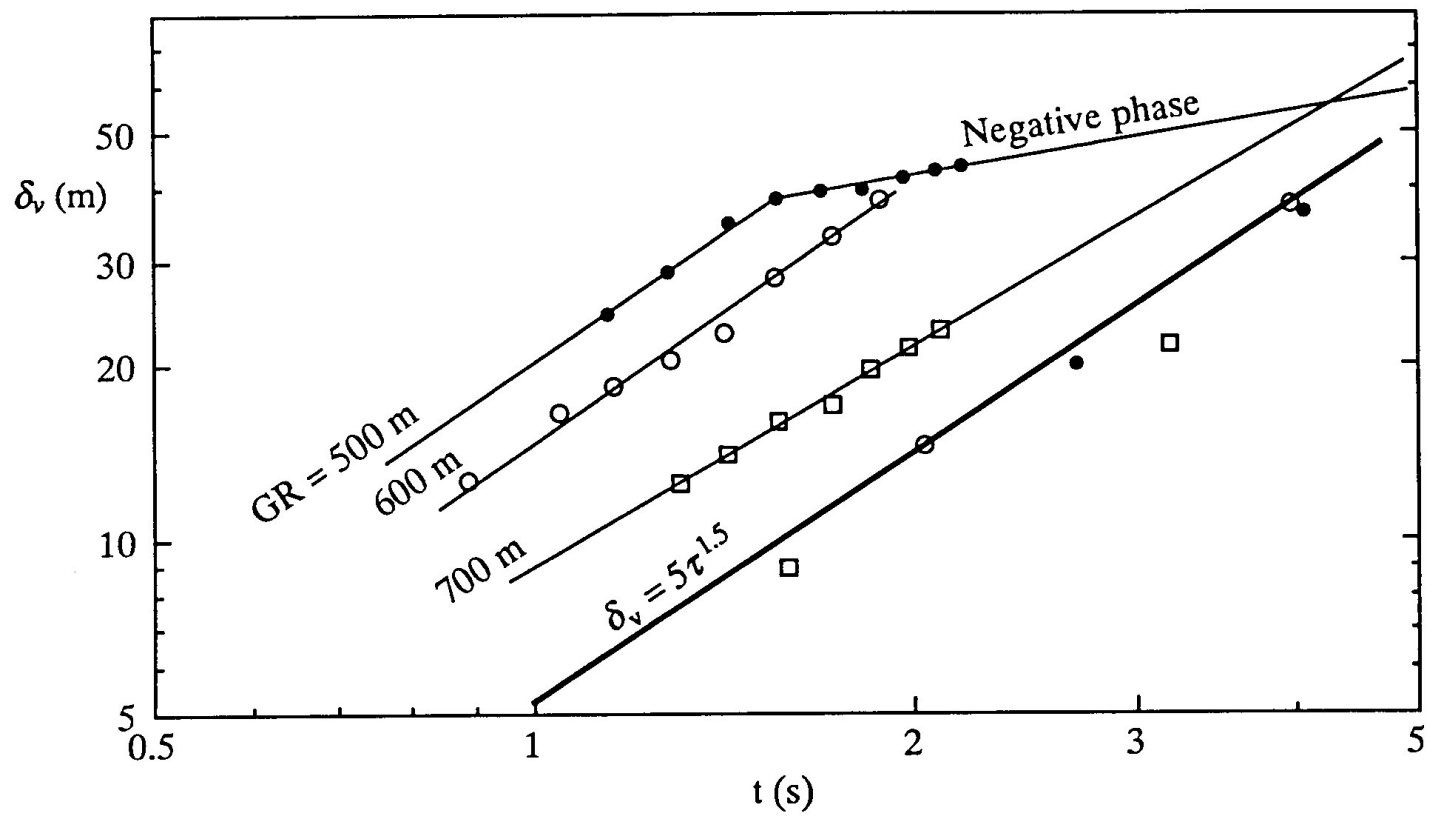

Figure 12. Dusty boundary layer growth.
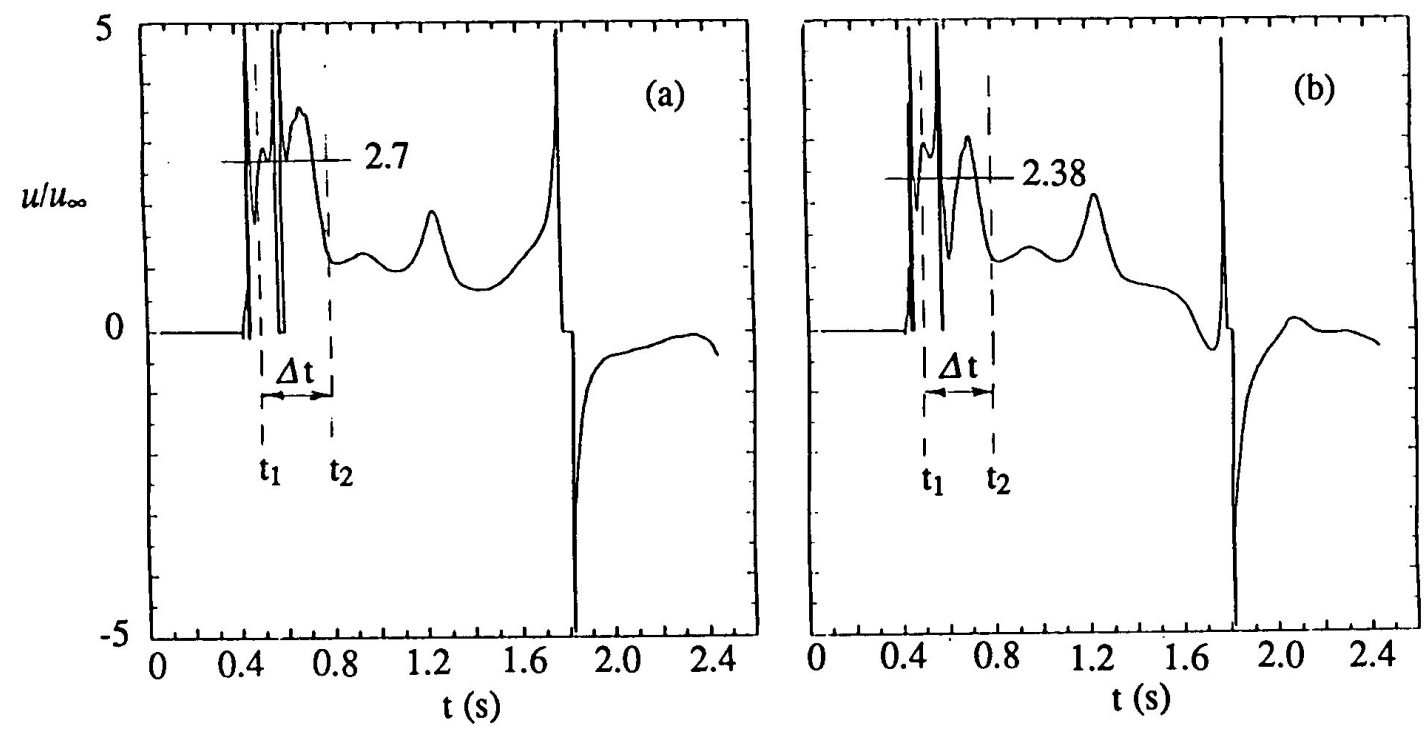

Figure 13. Nondimensional velocity histories at $\mathrm{GR}=600 \mathrm{~m}$ : (a) $y=0.8$; (b) $y=0.9$. 
Problem 3. Blast Wave Reflections from Ground Surfaces
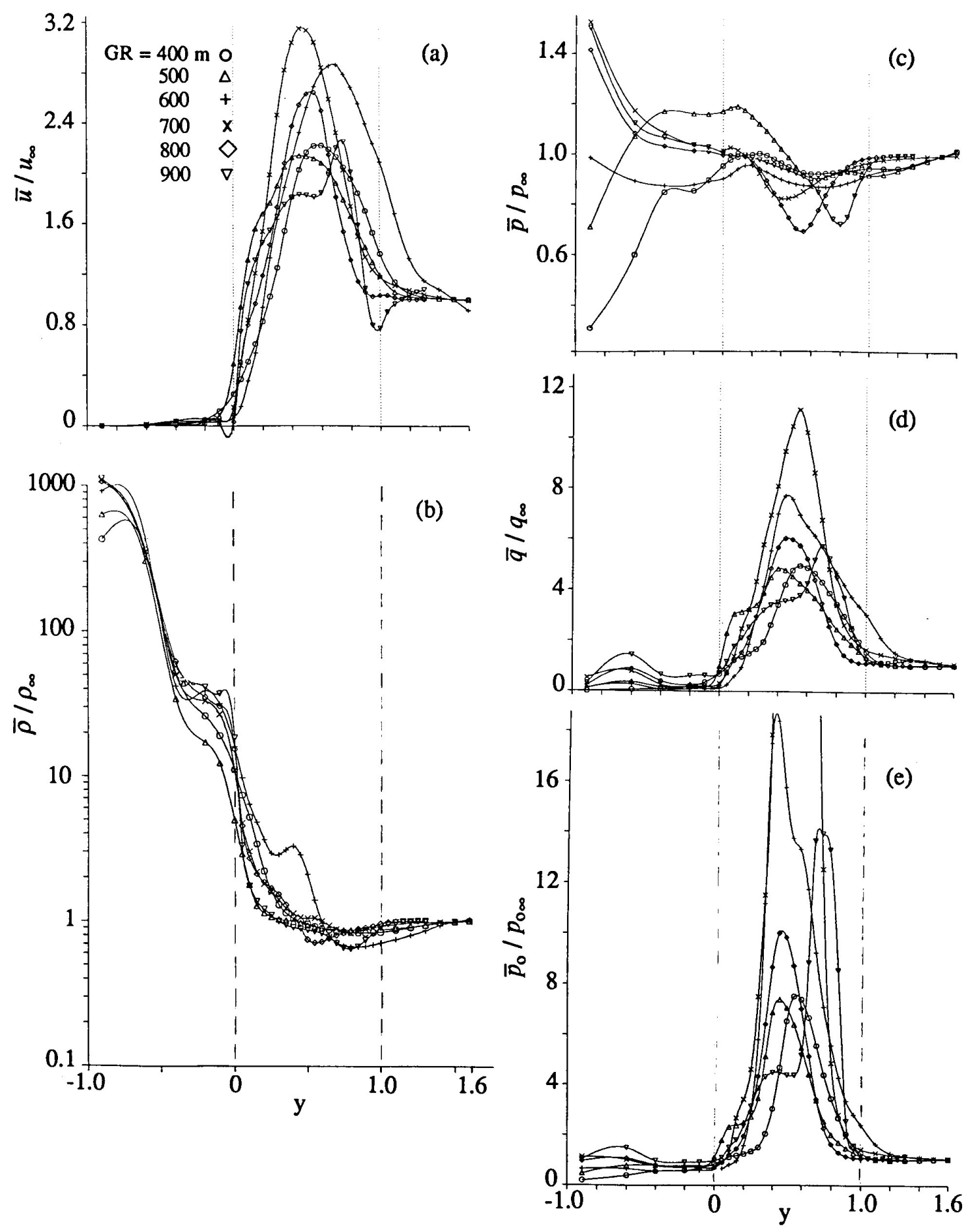

Figure 14. Free stream-scaled wall jet mean profiles: (a) streamwise velocity; (b) density; (c) pressure; (d) dynamic pressure; (e) pitot pressure. 
Problem 3. Blast Wave Reflections from Ground Surfaces
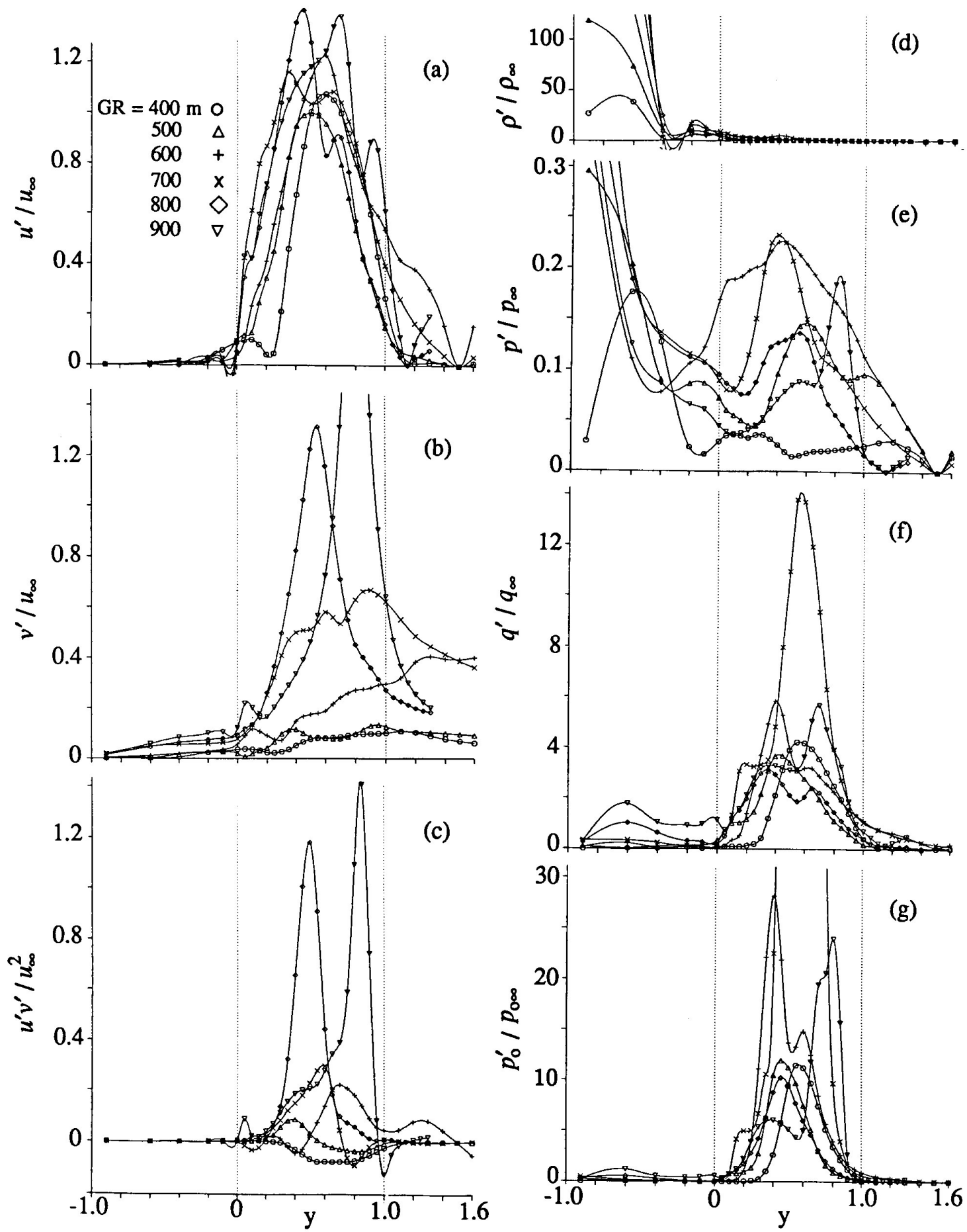

(b)
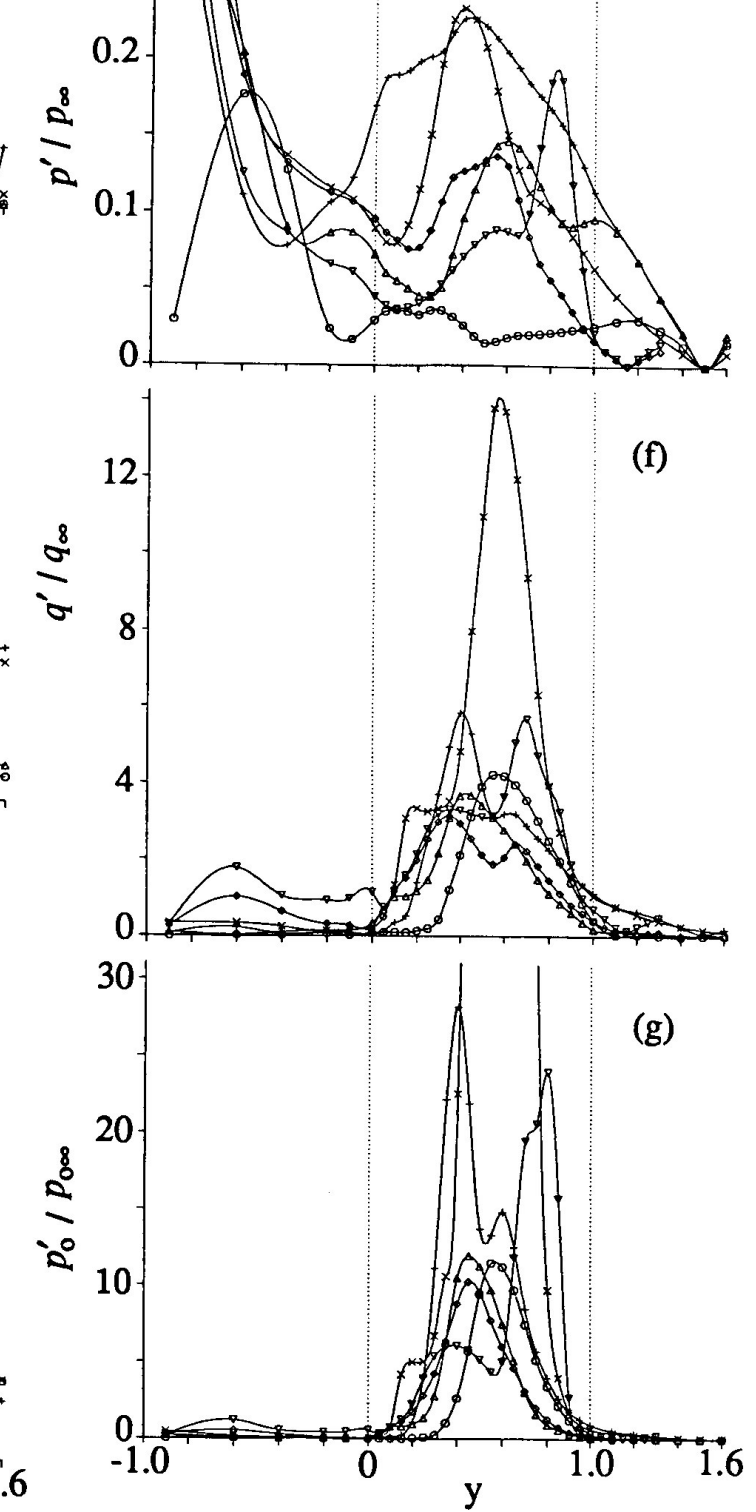

Figure 15. Free stream-scaled wall jet R.M.S. profiles: (a) streamwise velocity; (b) vertical velocity; (c) Reynolds stress; (d) density; (e) pressure;

(f) dynamic pressure; (g) pitot pressure. 


\section{Problem 3. Blast Wave Reflections from Ground Surfaces}
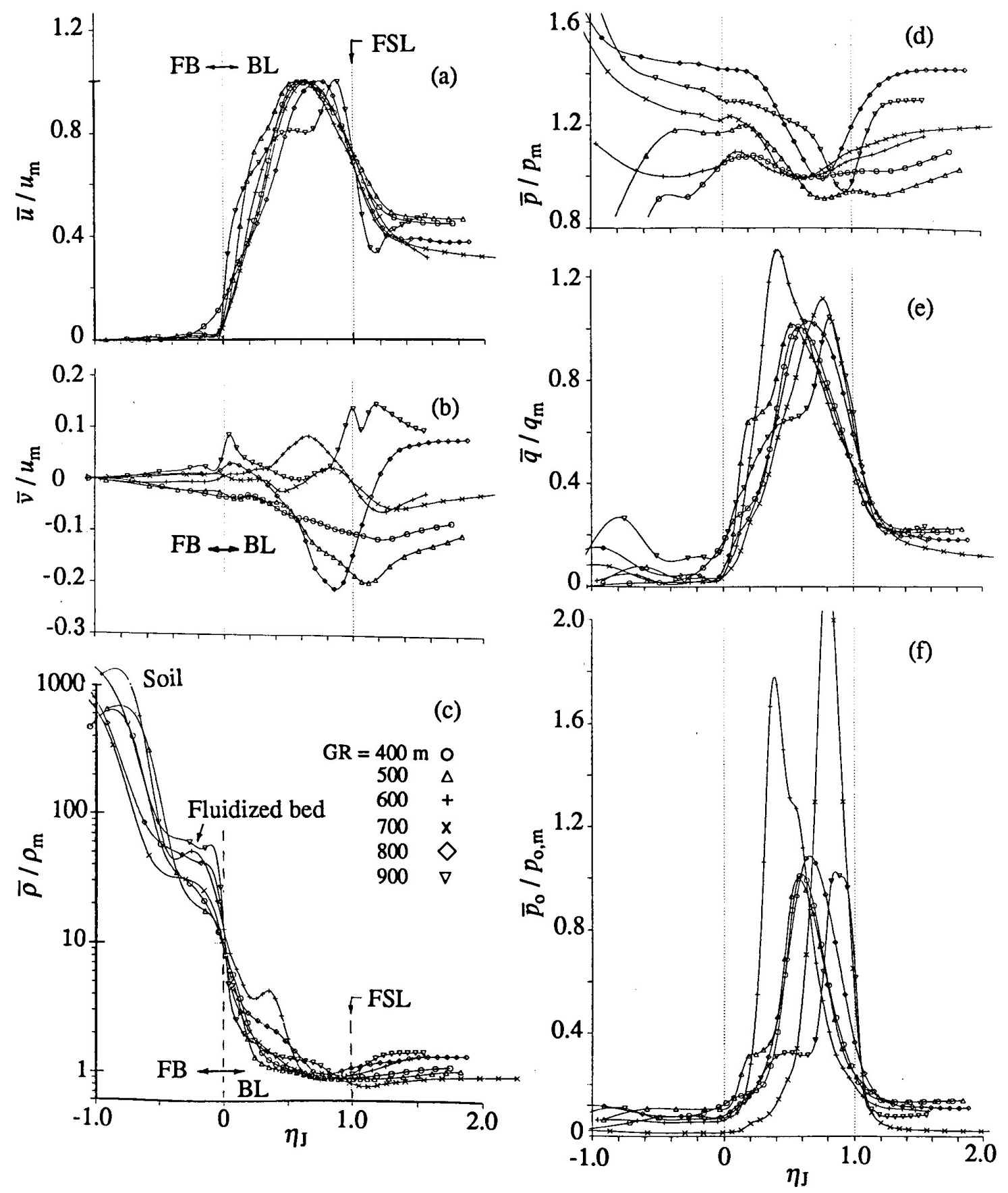

Figure 16. Wall-jet-scaled mean profiles: (a) streamwise velocity; (b) vertical velocity; (c) density; (d) pressure; (e) dynamic pressure; (f) pitot pressure. 


\section{Problem 3. Blast Wave Reflections from Ground Surfaces}
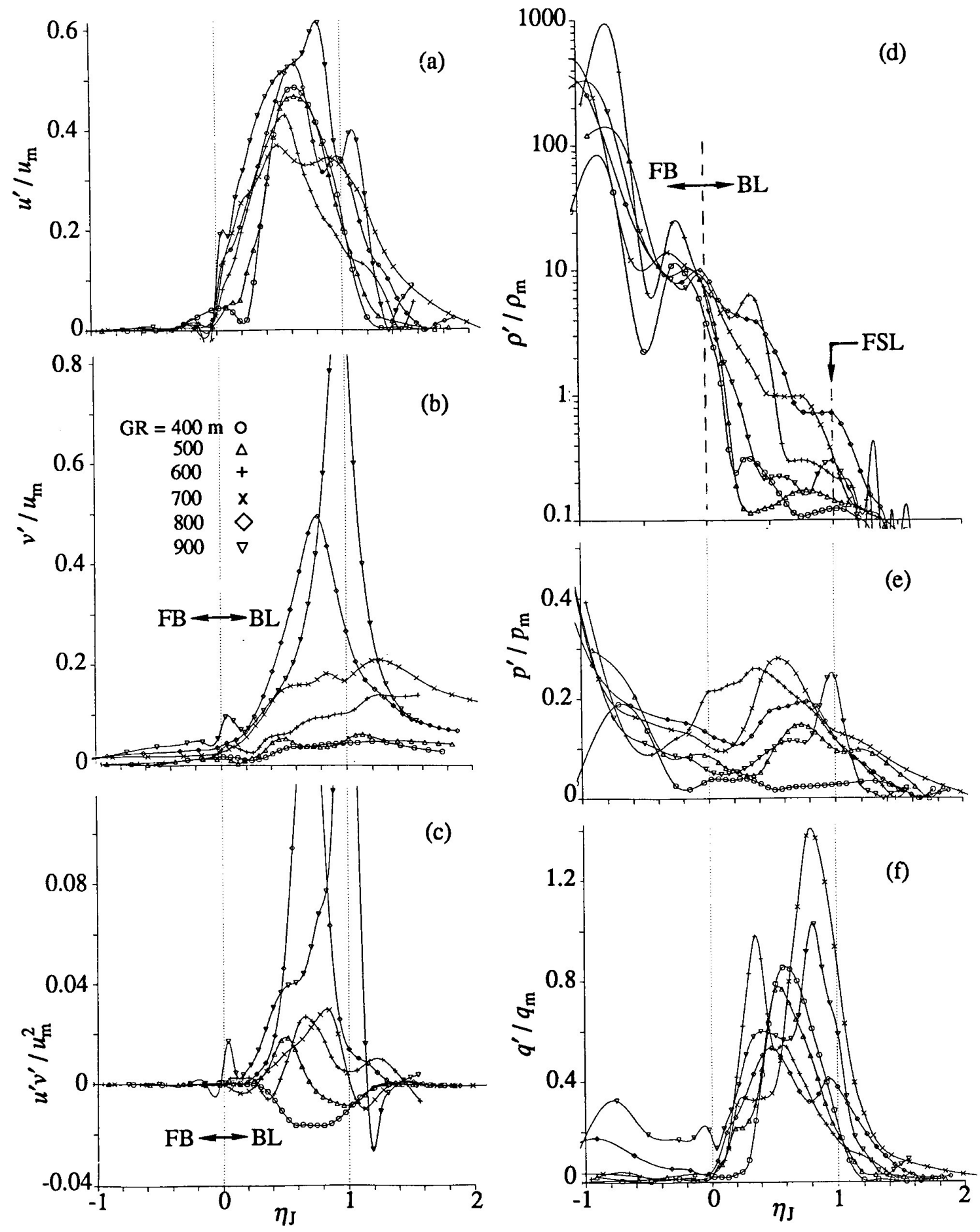

Figure 17. Wall-jet-scaled R.M.S. profiles: (a) streamwise velocity; (b) vertical velocity; (c) Reynolds stress; (d) density; (e) pressure; d) dynamic pressure. 
Problem 3. Blast Wave Reflections from Ground Surfaces
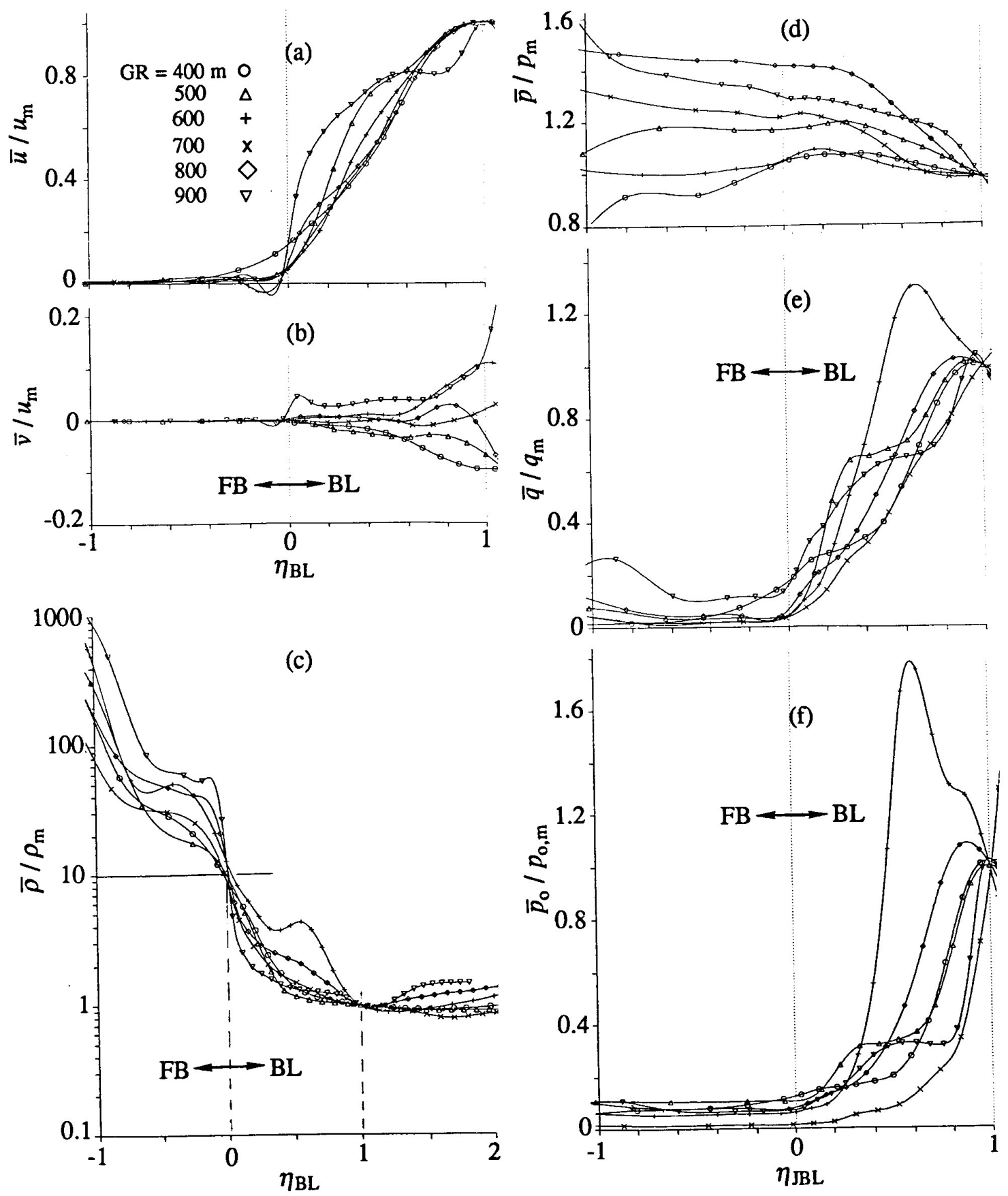

Figure 18. Mean boundary layer profiles of the wall jet: (a) streamwise velocity; (b) vertical velocity; (c) density; (d) pressure; (e) dynamic pressure; (f) pitot pressure.

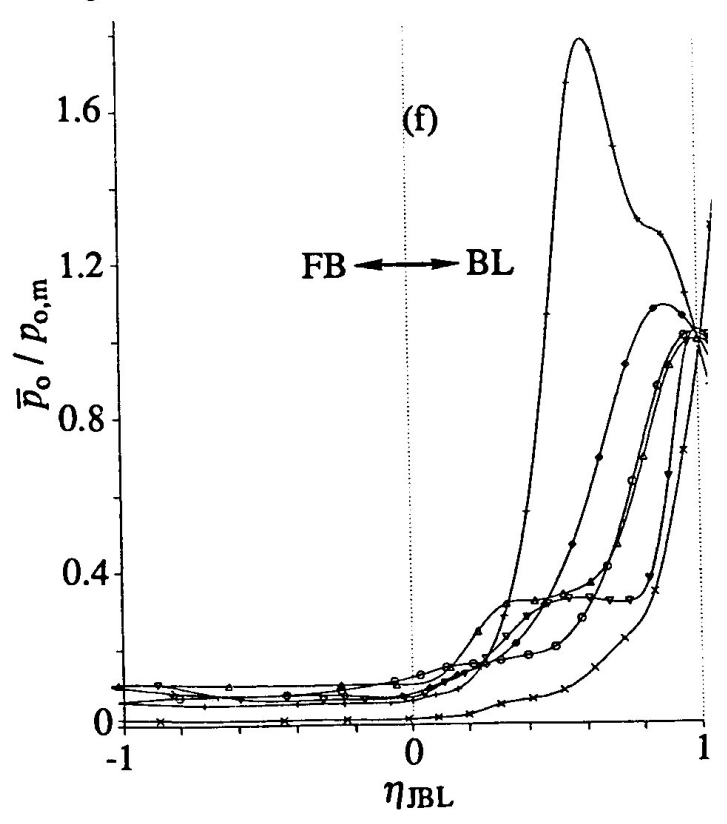




\section{Problem 3. Blast Wave Reflections from Ground Surfaces}
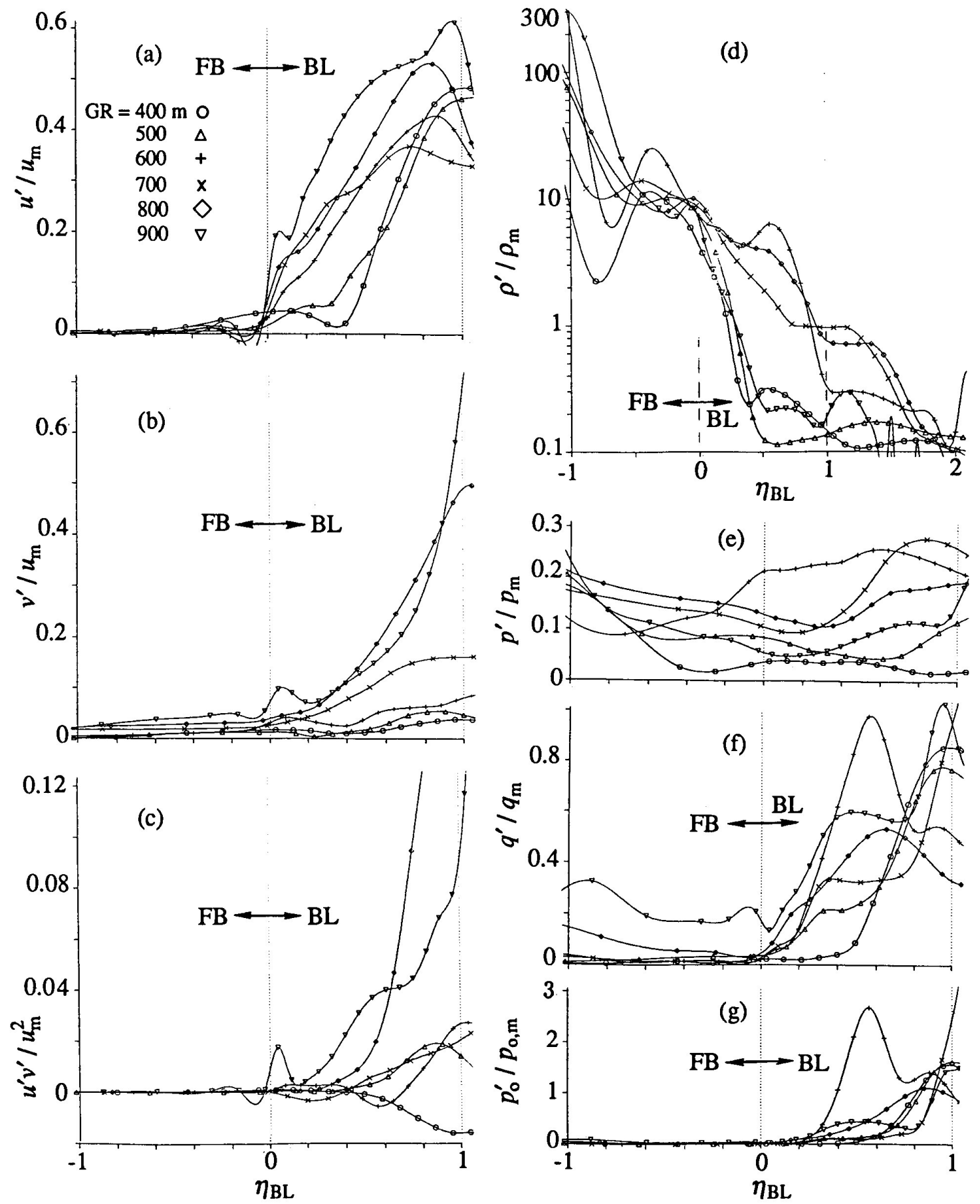

Figure 19. R.M.S. boundary layer profiles of the wall jet: (a) streamwise velocity;

(b) vertical velocity; (c) Reynolds stress; (d) density; (e) pressure;

(f) dynamic pressure; (g) pitot pressure. 


\section{Problem 3. Blast Wave Reflections from Ground Surfaces}

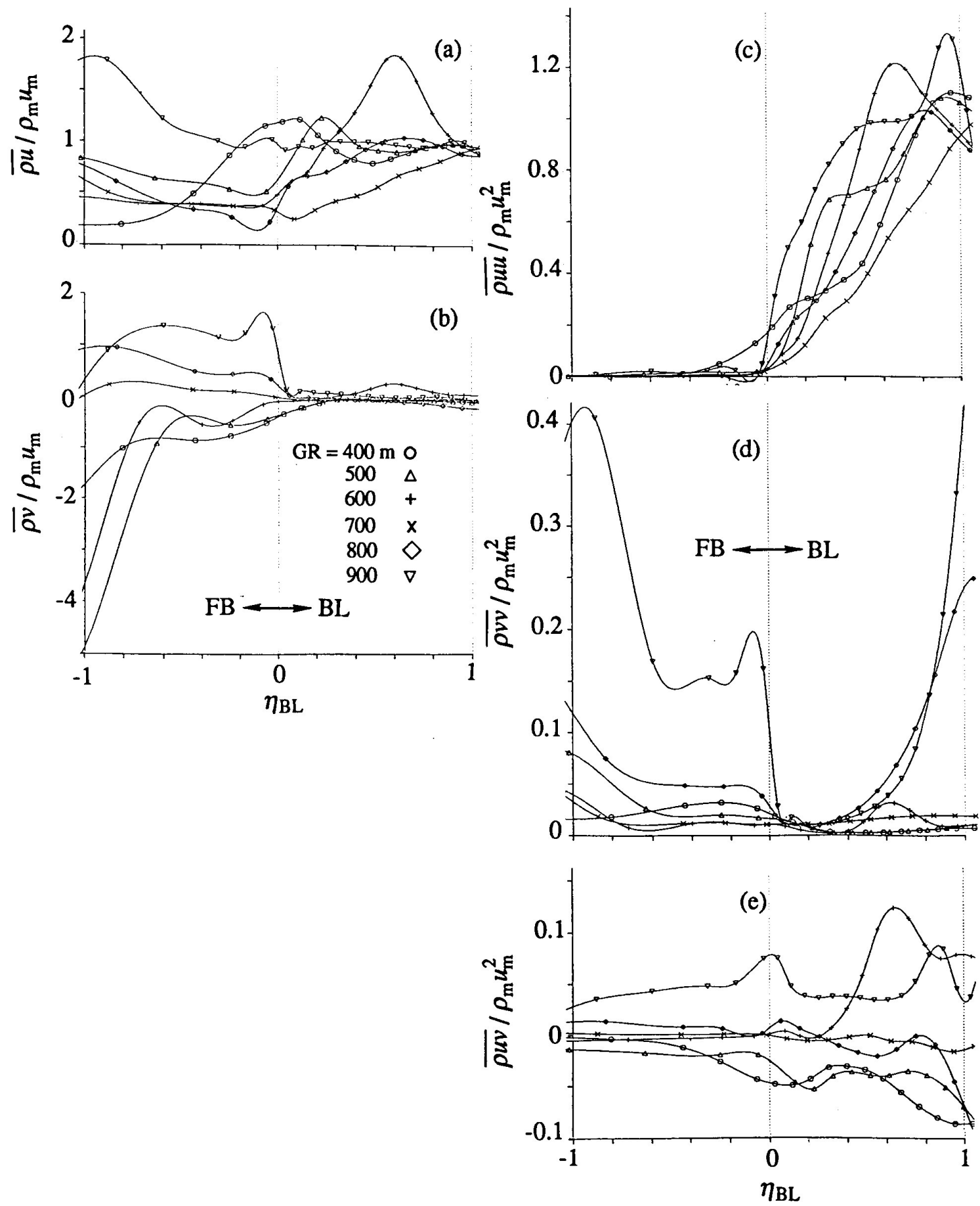

Figure 20. Mean boundary layer flux profiles of the wall jet: (a) streamwise mass;

(b) vertical mass; (c) streamwise momentum; (d) vertical momentum;

(e) cross-stream momentum. 
Problem 3. Blast Wave Reflections from Ground Surfaces
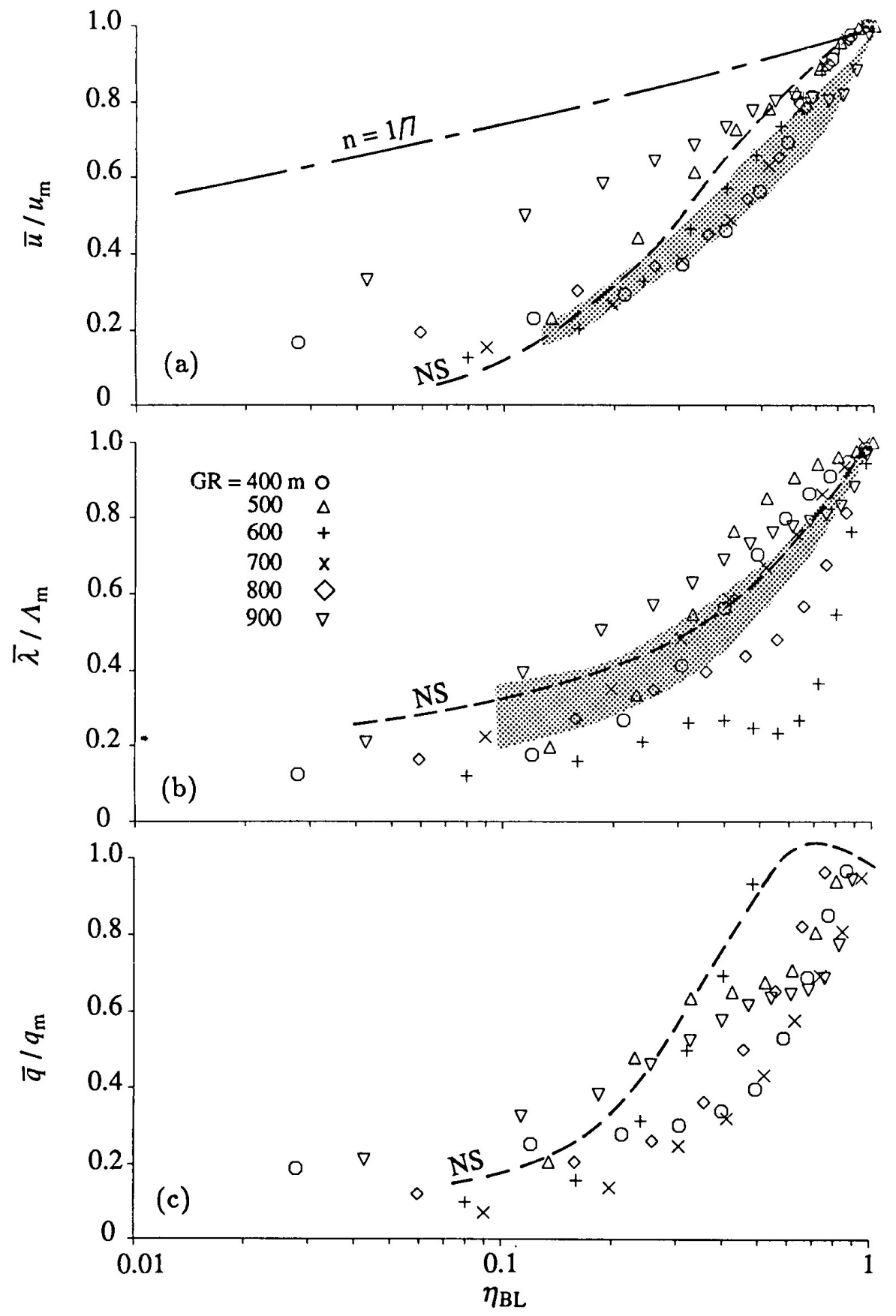

Figure 21. Mean boundary layer profiles of the wall jet: (a) streamwise velocity; (b) specific volume; (c) dynamic pressure. NS represents previous calculations ${ }^{8}$ for a normal shock, while shaded regions denote the data bands of Batt ${ }^{18}$. 

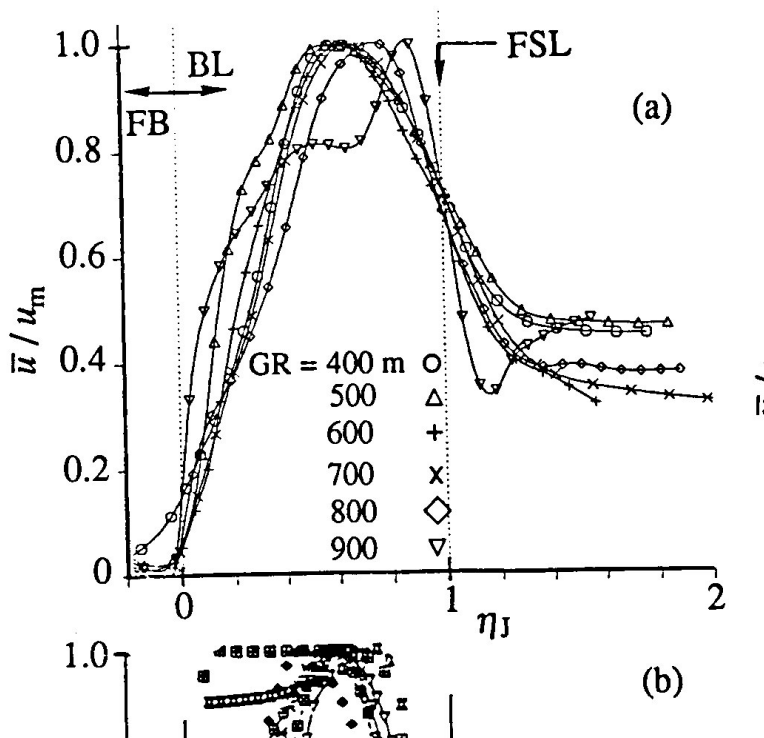

(b)
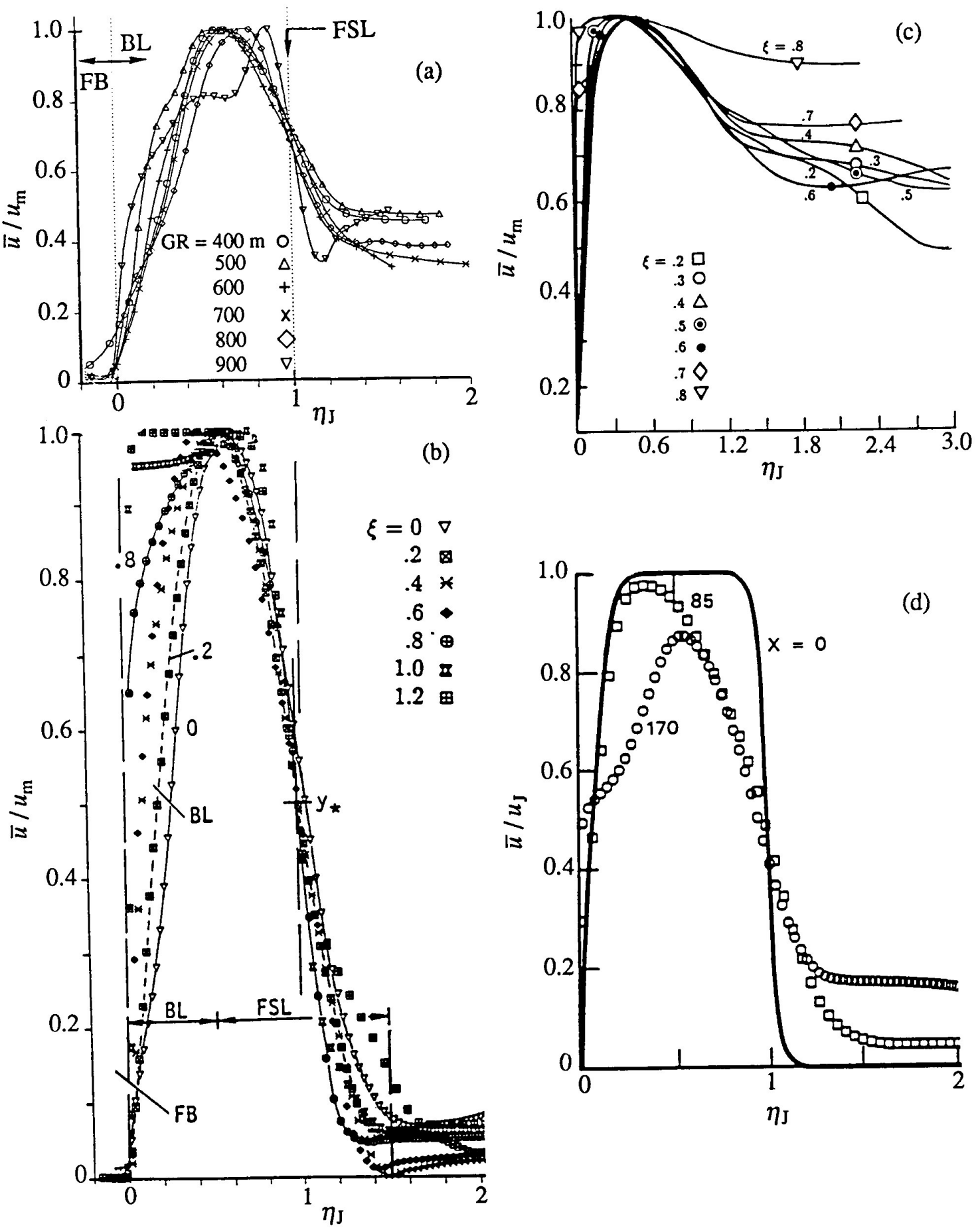

Figure 22. Comparison of mean wall jet velocity profiles: (a) present blast wave precursor calculation; (b) square-wave precursor; (c) DMR wall jet;

(d) steady-flow wall jet. 

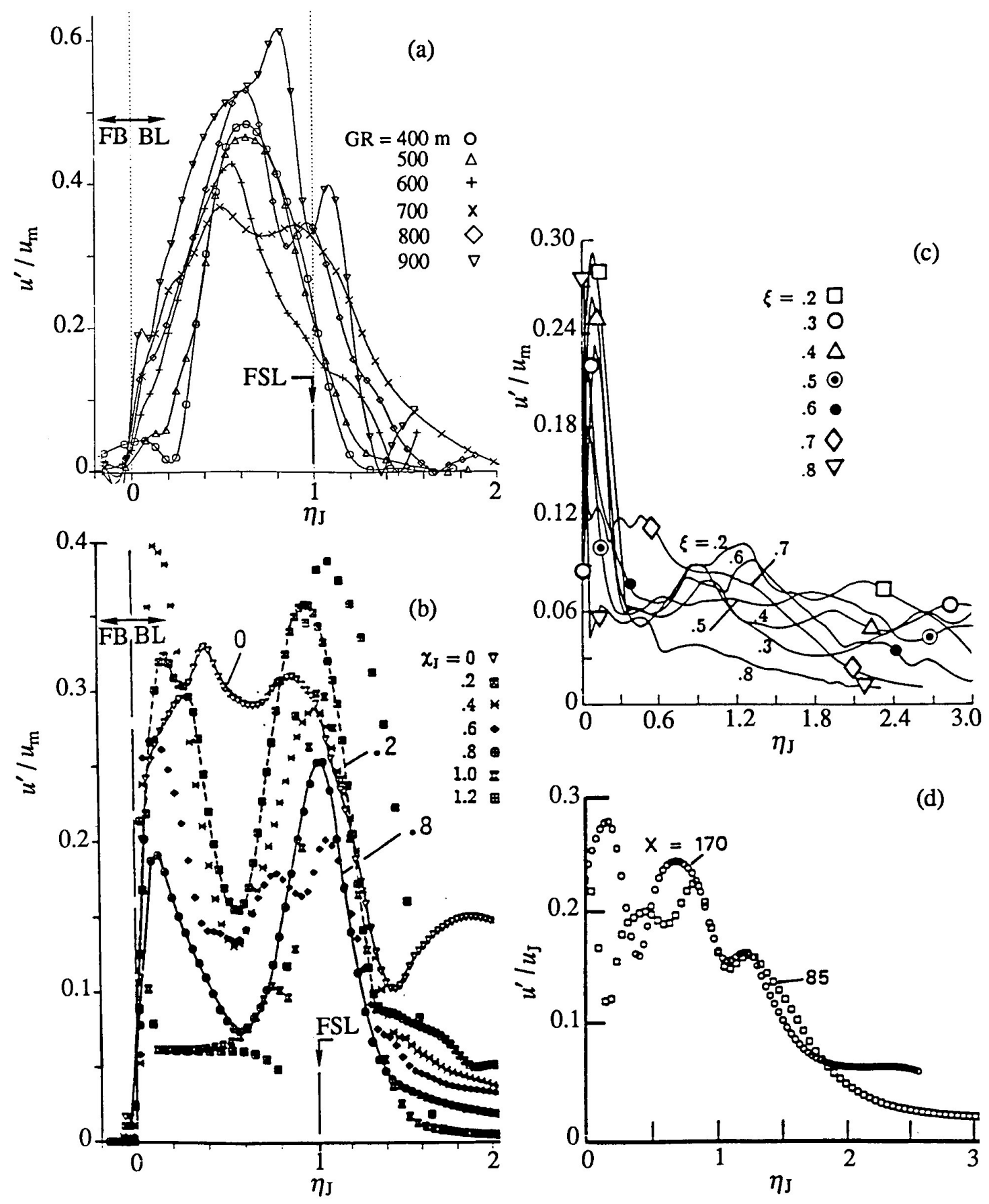

Figure 23. Comparison of wall jet $u^{\prime}$ fluctuation profiles: (a) present blast wave precursor calculation; (b) square-wave precursor; (c) DMR wall jet; (d) steady-flow wall jet. 
Problem 3. Blast Wave Reflections from Ground Surfaces
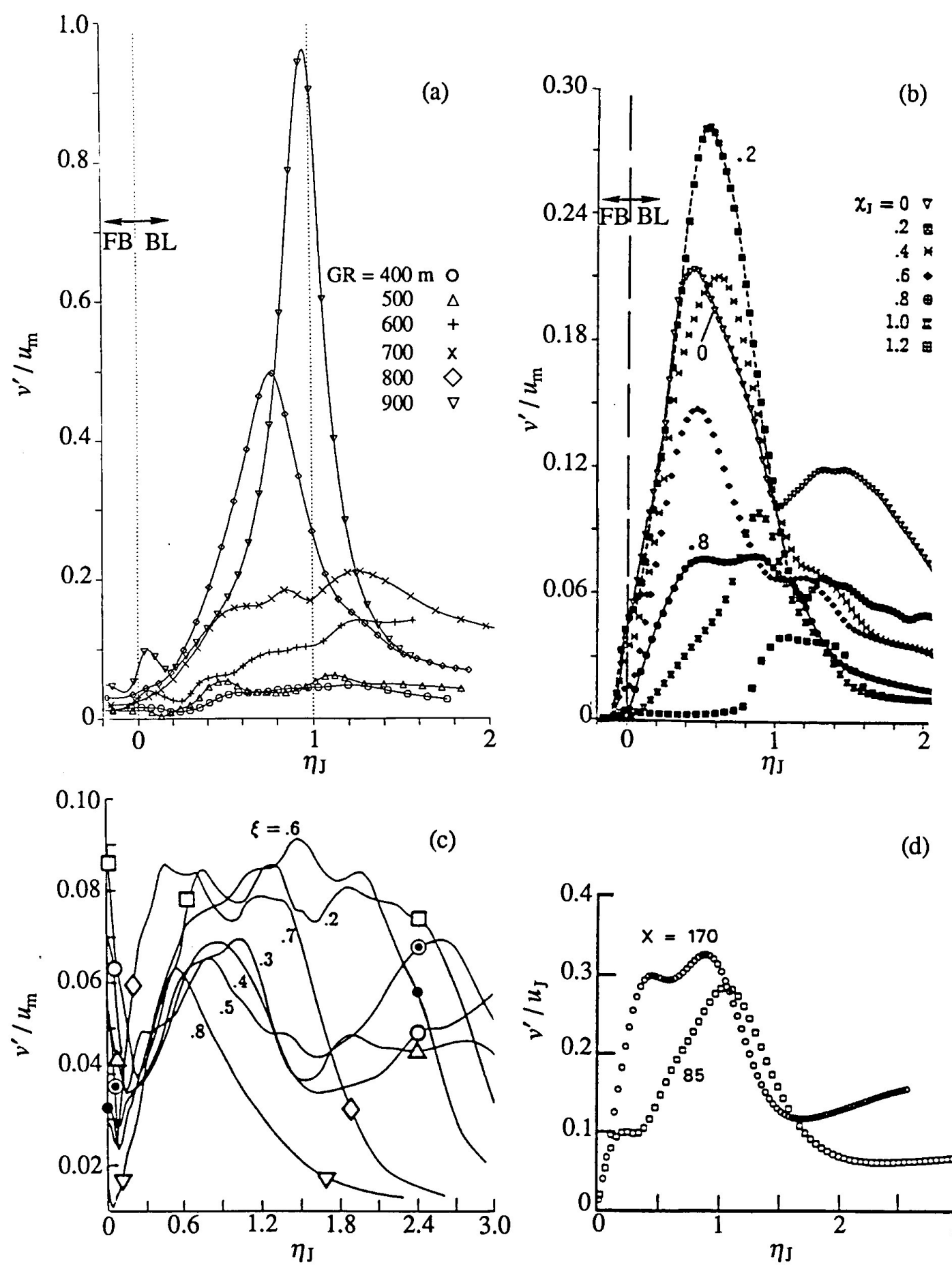

Figure 24. Comparison of wall jet $v^{\prime}$ fluctuation profiles: (a) present blast wave precursor calculation; (b) square-wave precursor; (c) DMR wall jet; (d) steady-flow wall jet.

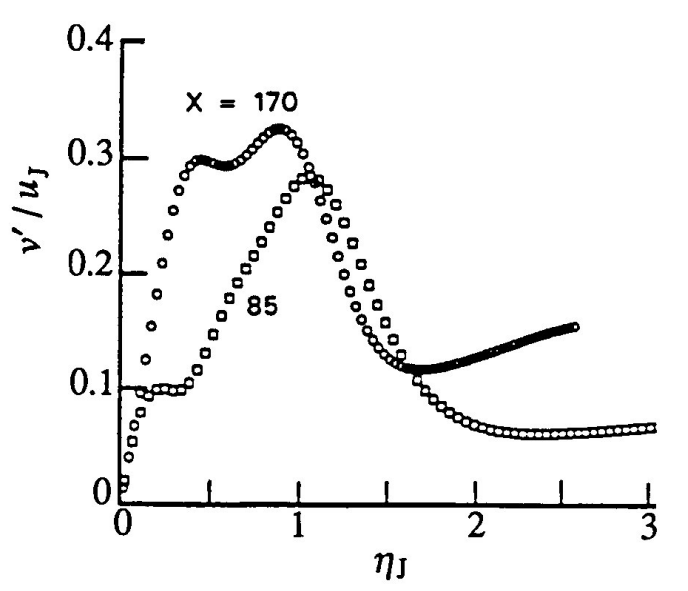


Problem 3. Blast Wave Reflections from Ground Surfaces
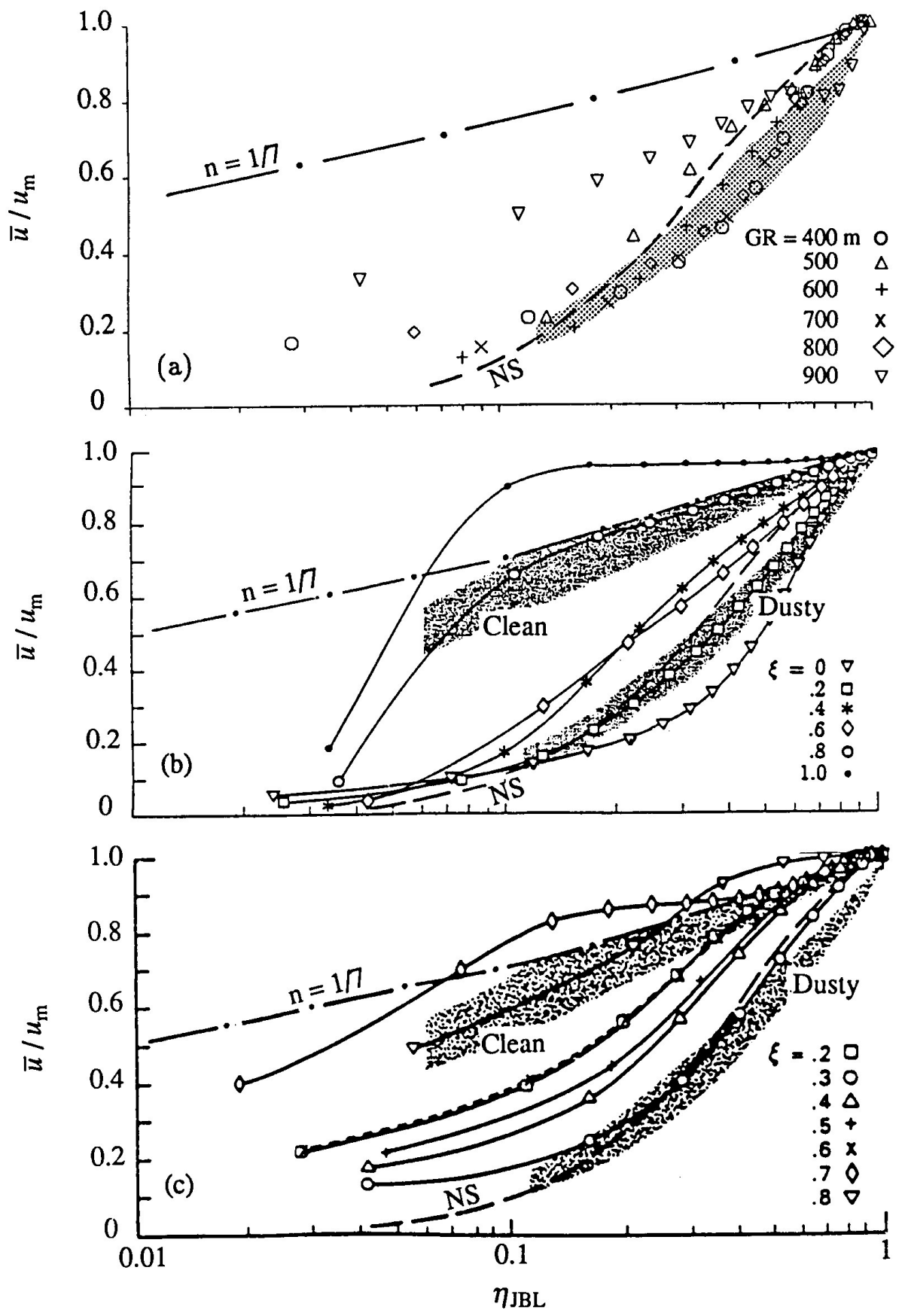

Figure 25. Comparison of wall jet mean velocity profiles: (a) present blast wave precursor calculation; (b) square-wave precursor; (c) DMR wall jet. 


\section{Problem 3. Blast Wave Reflections from Ground Surfaces}

Appendix A: Empirical thermal layer from Event Priscilla

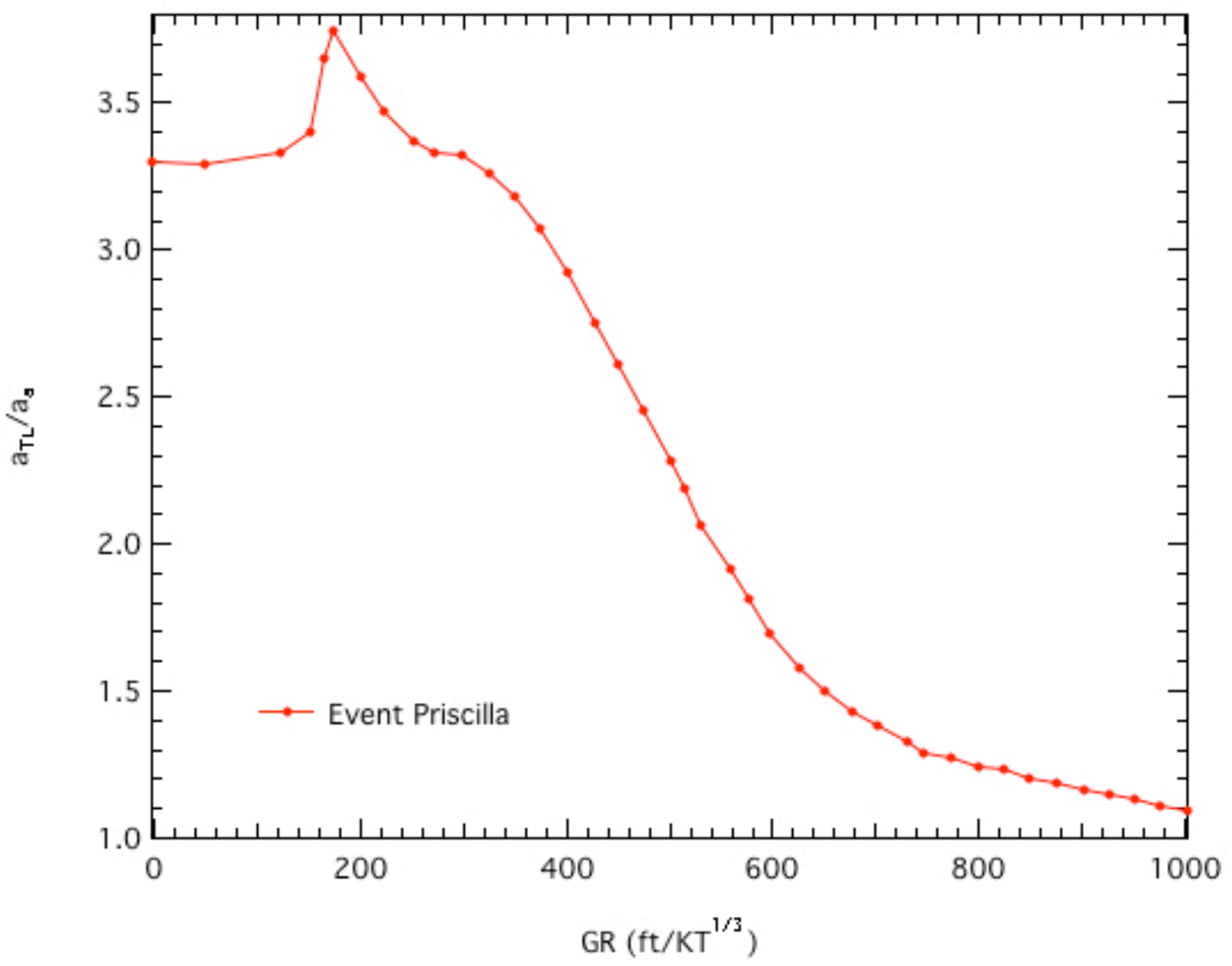




\section{Problem 3. Blast Wave Reflections from Ground Surfaces}

\section{Appendix B: Color visualization of the precursor wall jet flow}

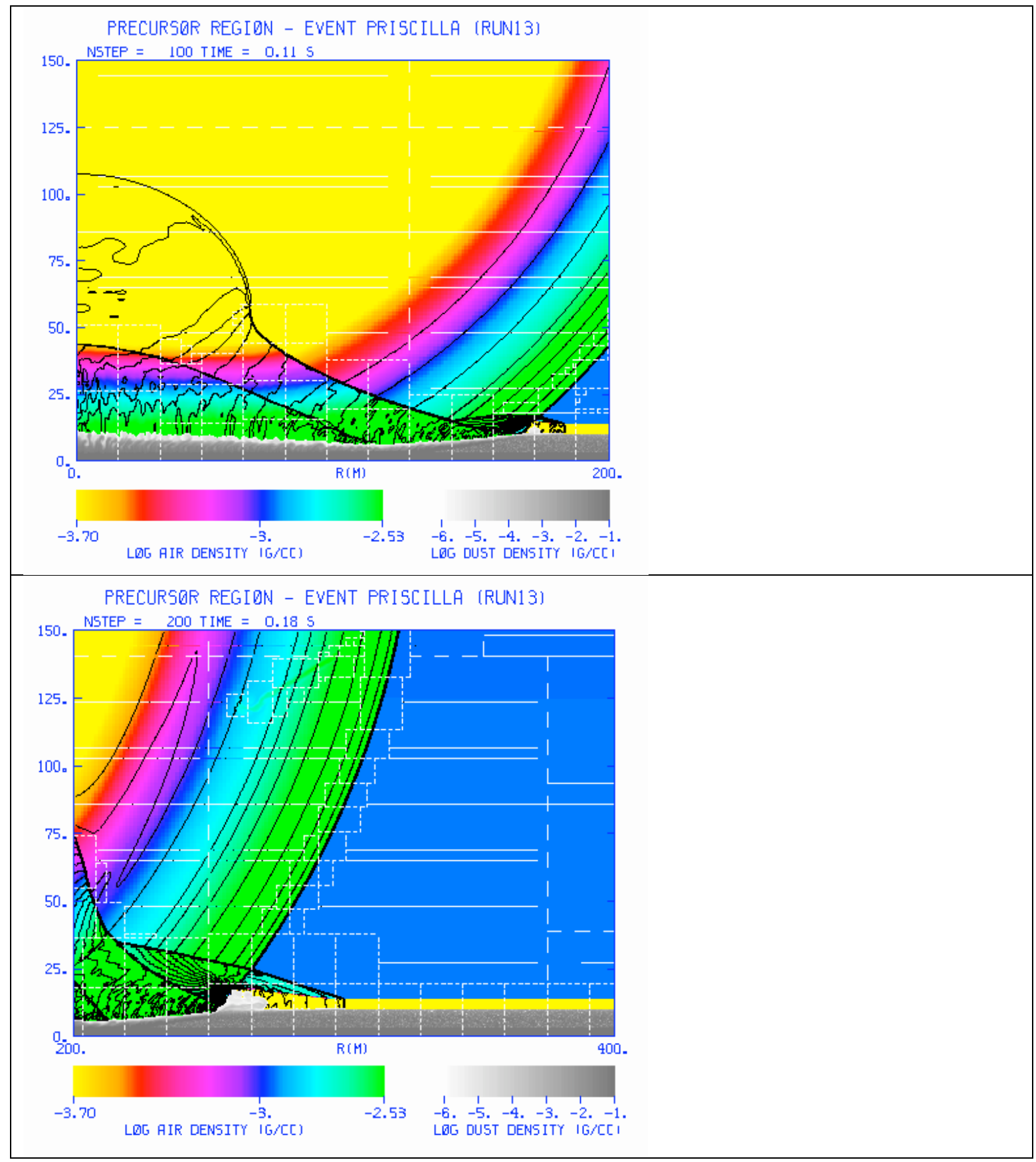




\section{Problem 3. Blast Wave Reflections from Ground Surfaces}

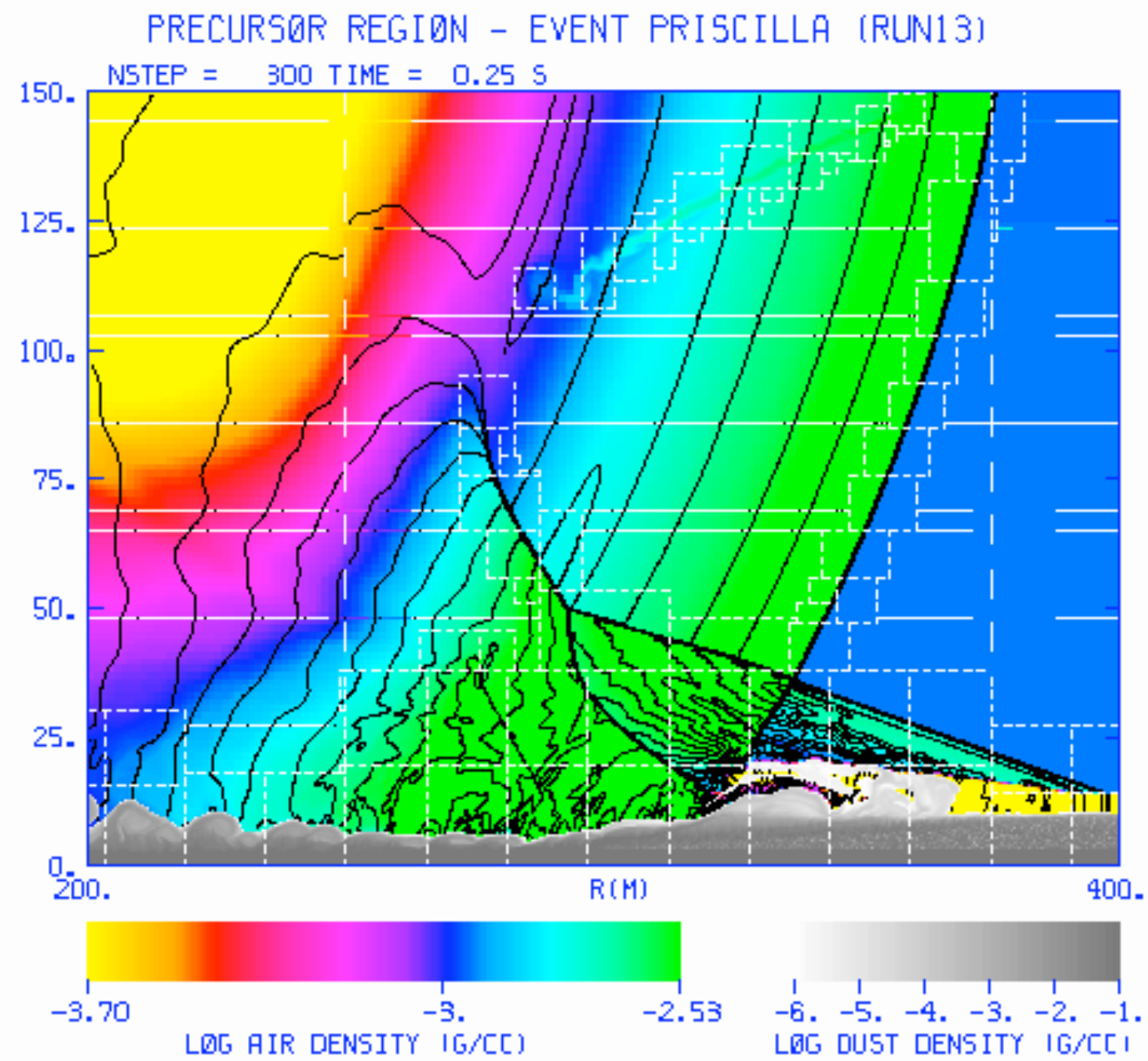


Problem 3. Blast Wave Reflections from Ground Surfaces

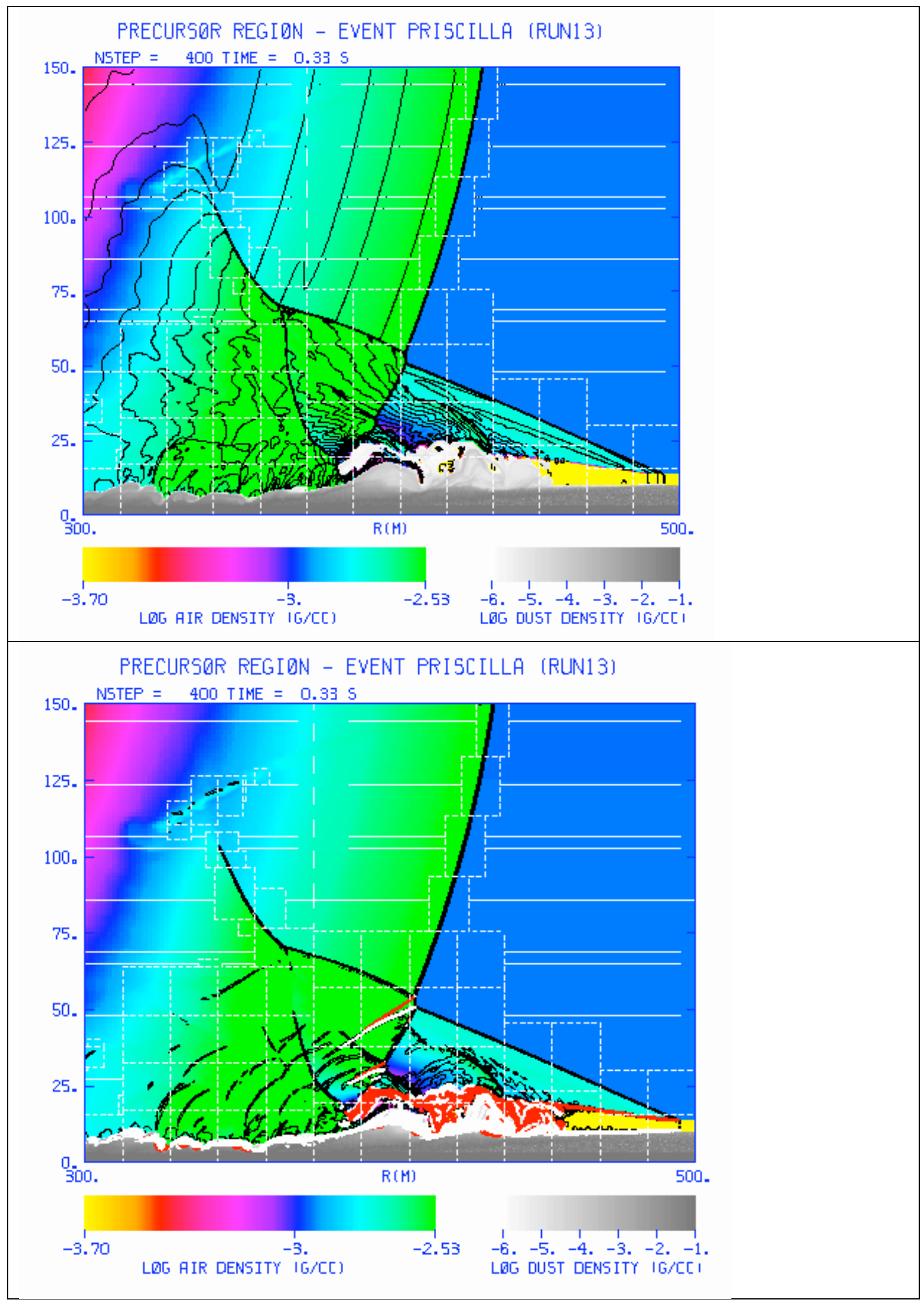


Problem 3. Blast Wave Reflections from Ground Surfaces

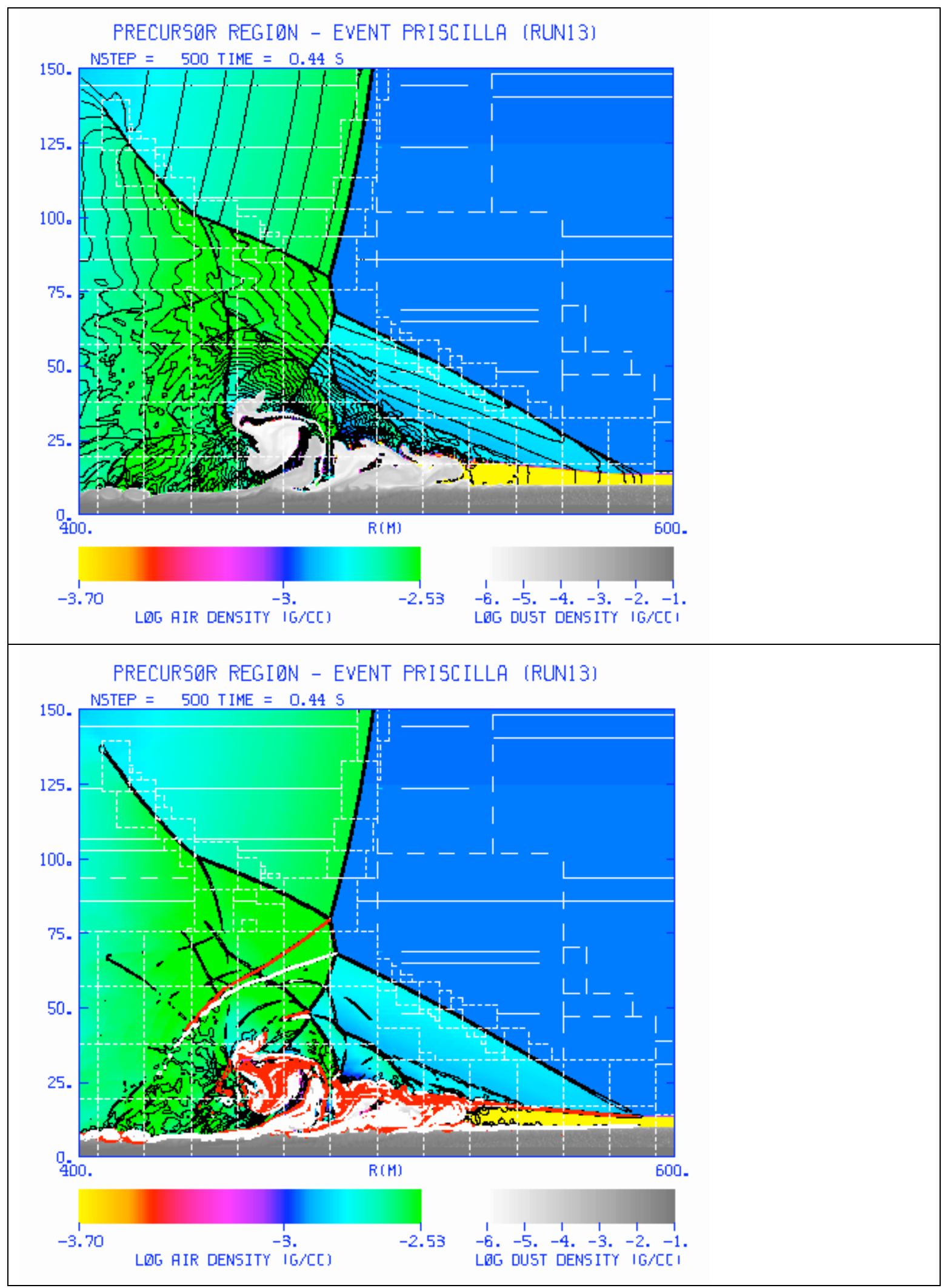


Problem 3. Blast Wave Reflections from Ground Surfaces

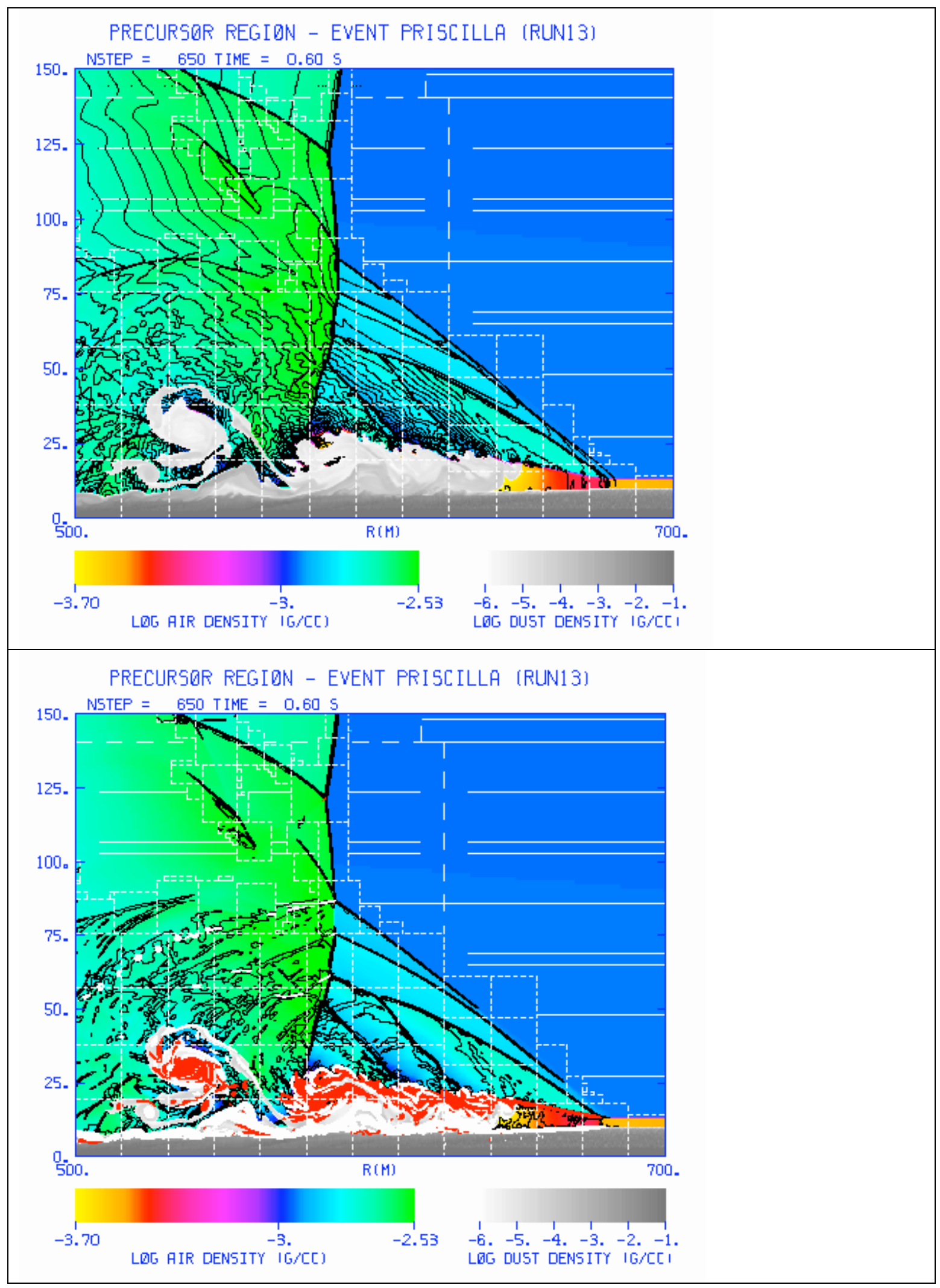


Problem 3. Blast Wave Reflections from Ground Surfaces

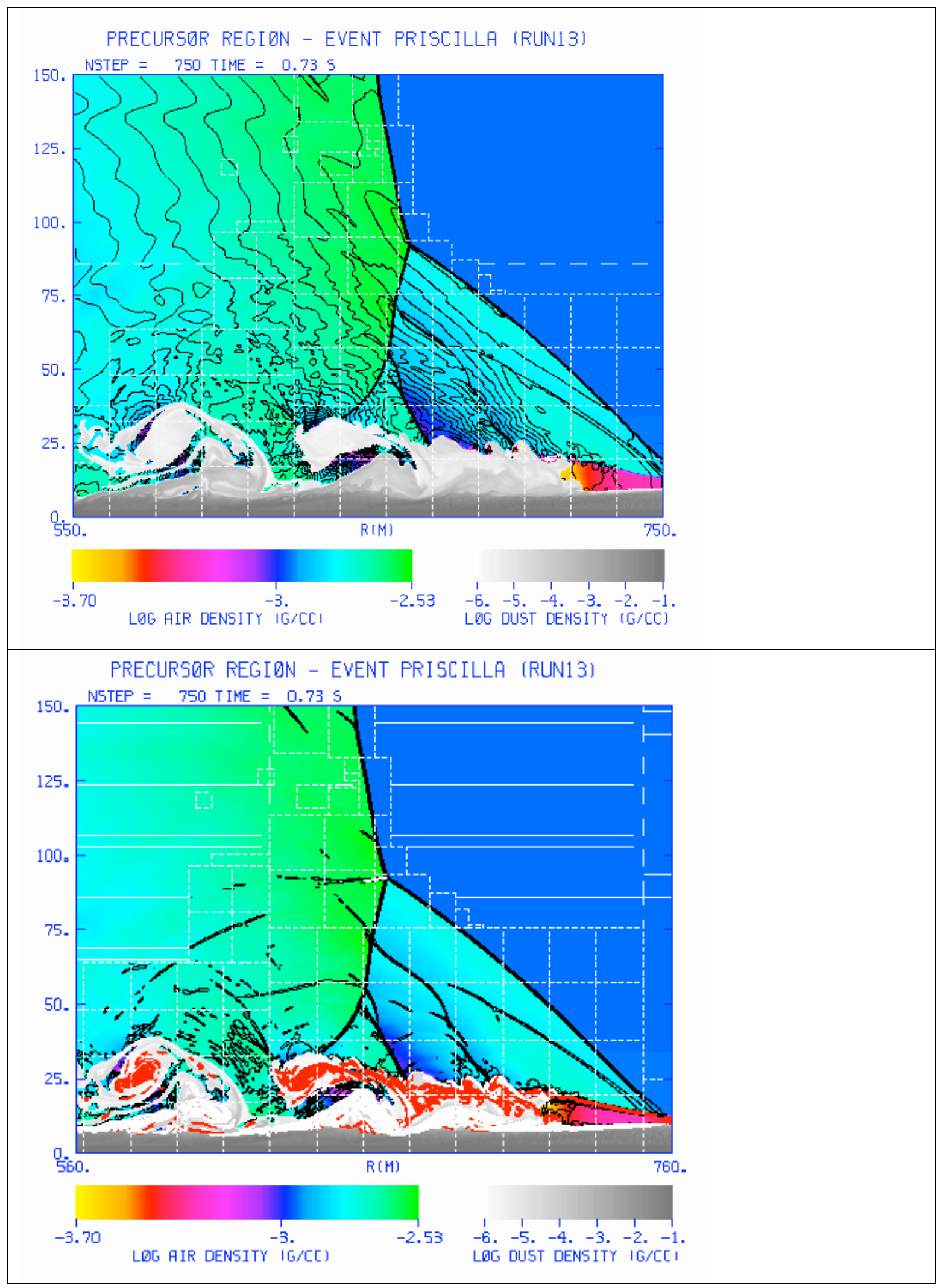




\section{Problem 3. Blast Wave Reflections from Ground Surfaces}

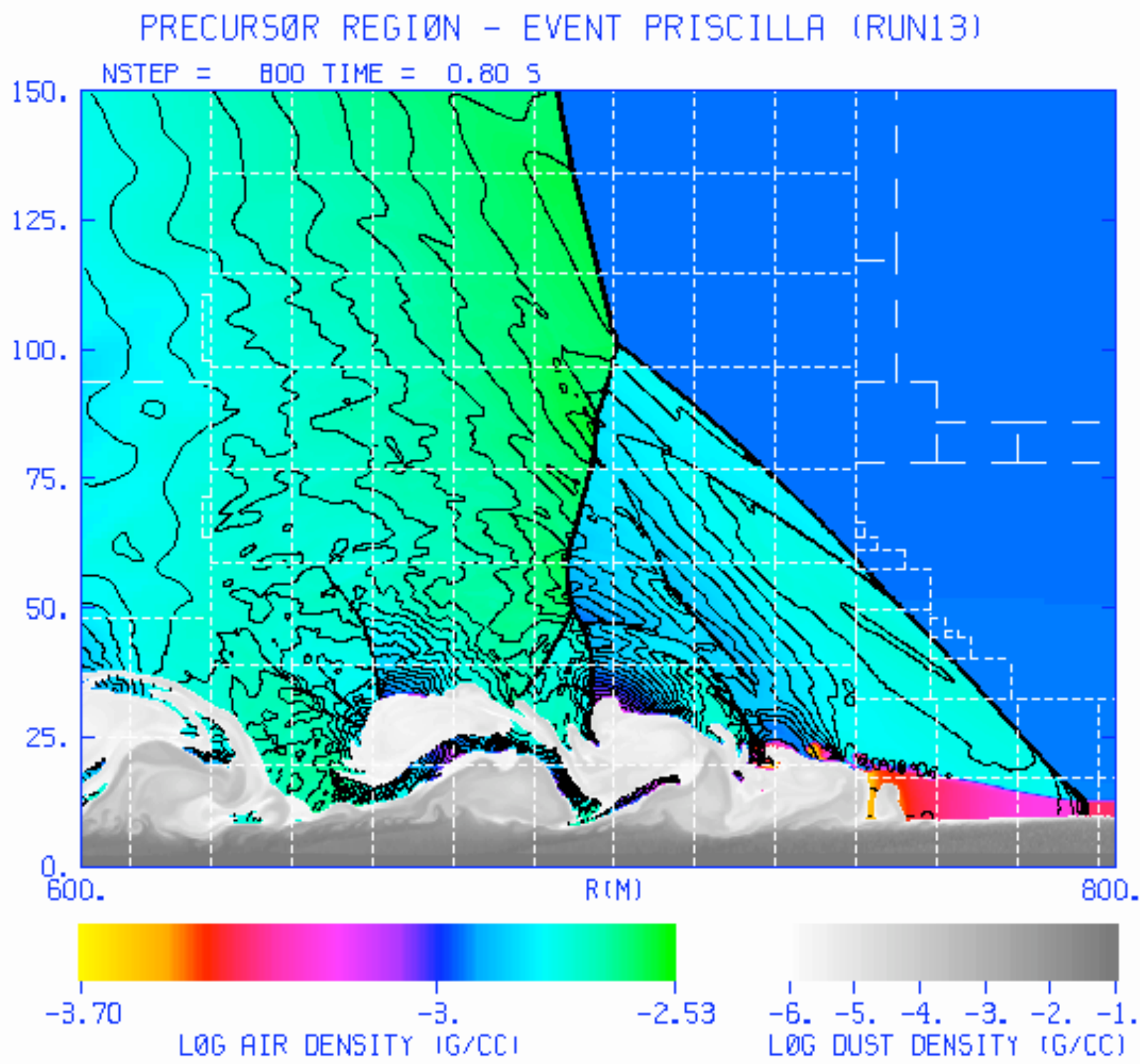




\section{Problem 3. Blast Wave Reflections from Ground Surfaces}

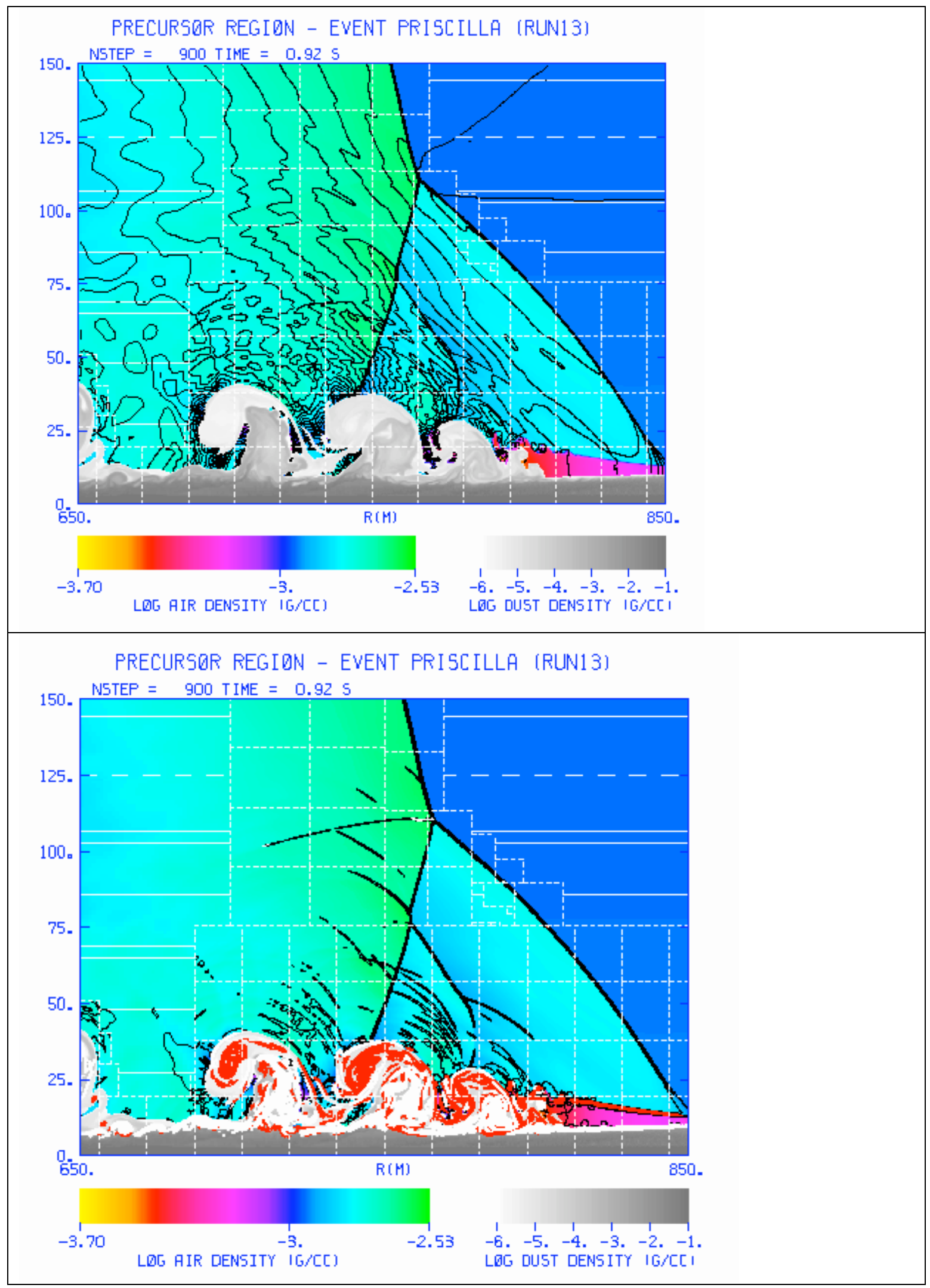


Problem 3. Blast Wave Reflections from Ground Surfaces

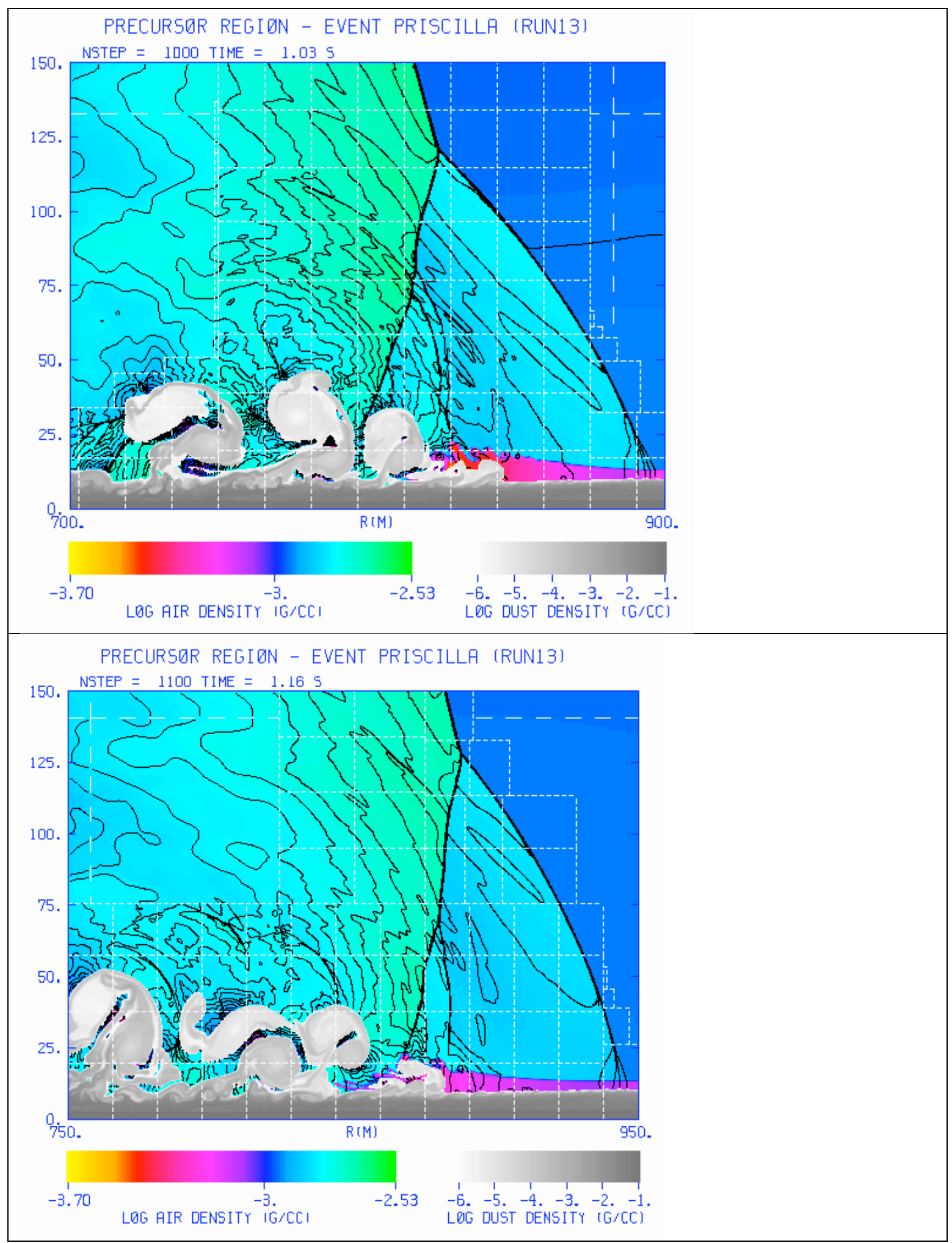




\section{Problem 3. Blast Wave Reflections from Ground Surfaces}

\section{Appendix C. Color visualization of the dusty boundary layer surface}
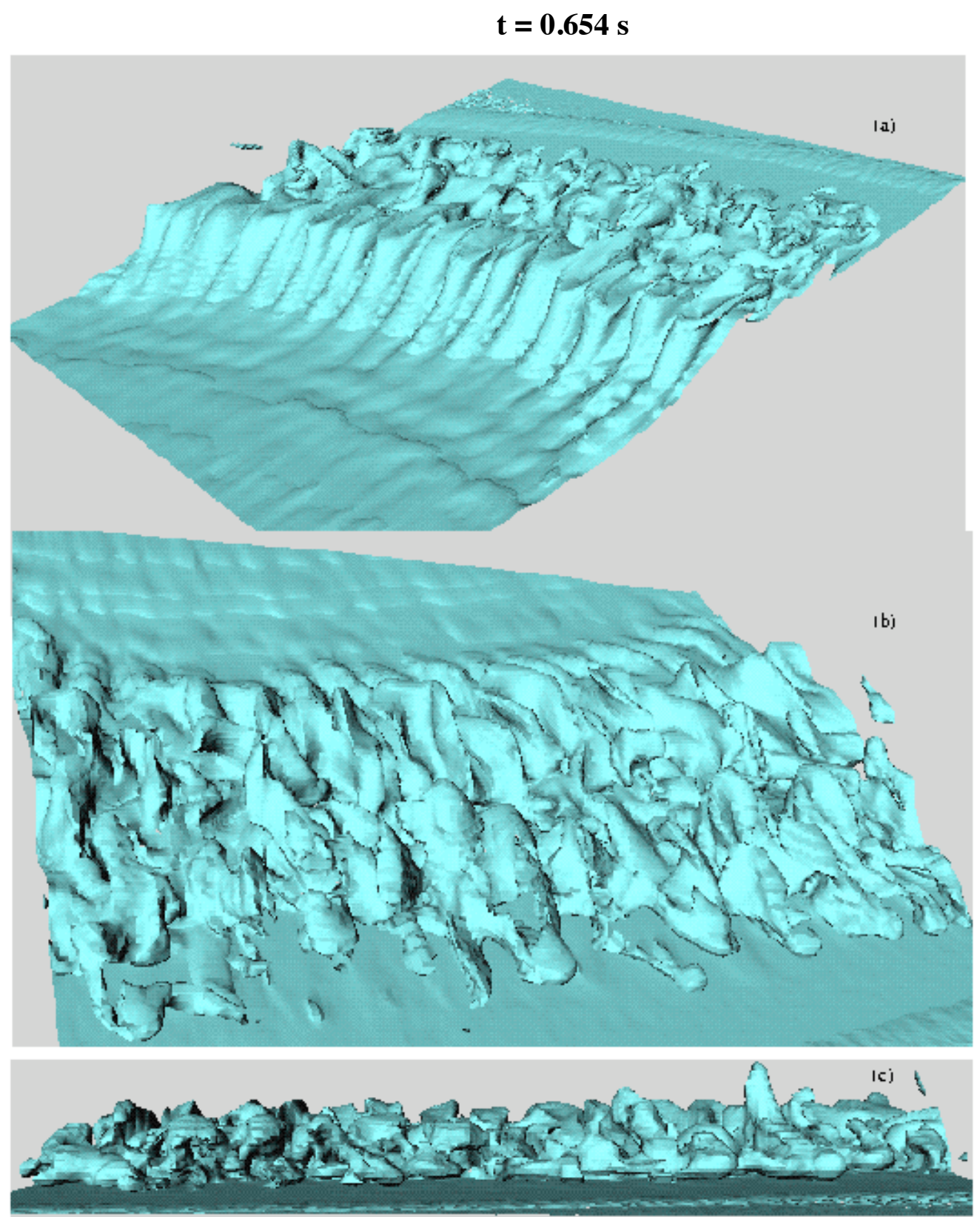

Thee-dimersional risualization of the turbulent wall jet and dusty bounday hyer flow for Pliscilla at $0.65+\mathrm{s}$ for $+56 m<x<720 m$ of dist concentuation of $10 \%$; l j oblique viewed, ibj riewed looking towad ground zero from above and icj riewed looking towad ground zeio at ground level. 


\section{Problem 3. Blast Wave Reflections from Ground Surfaces}
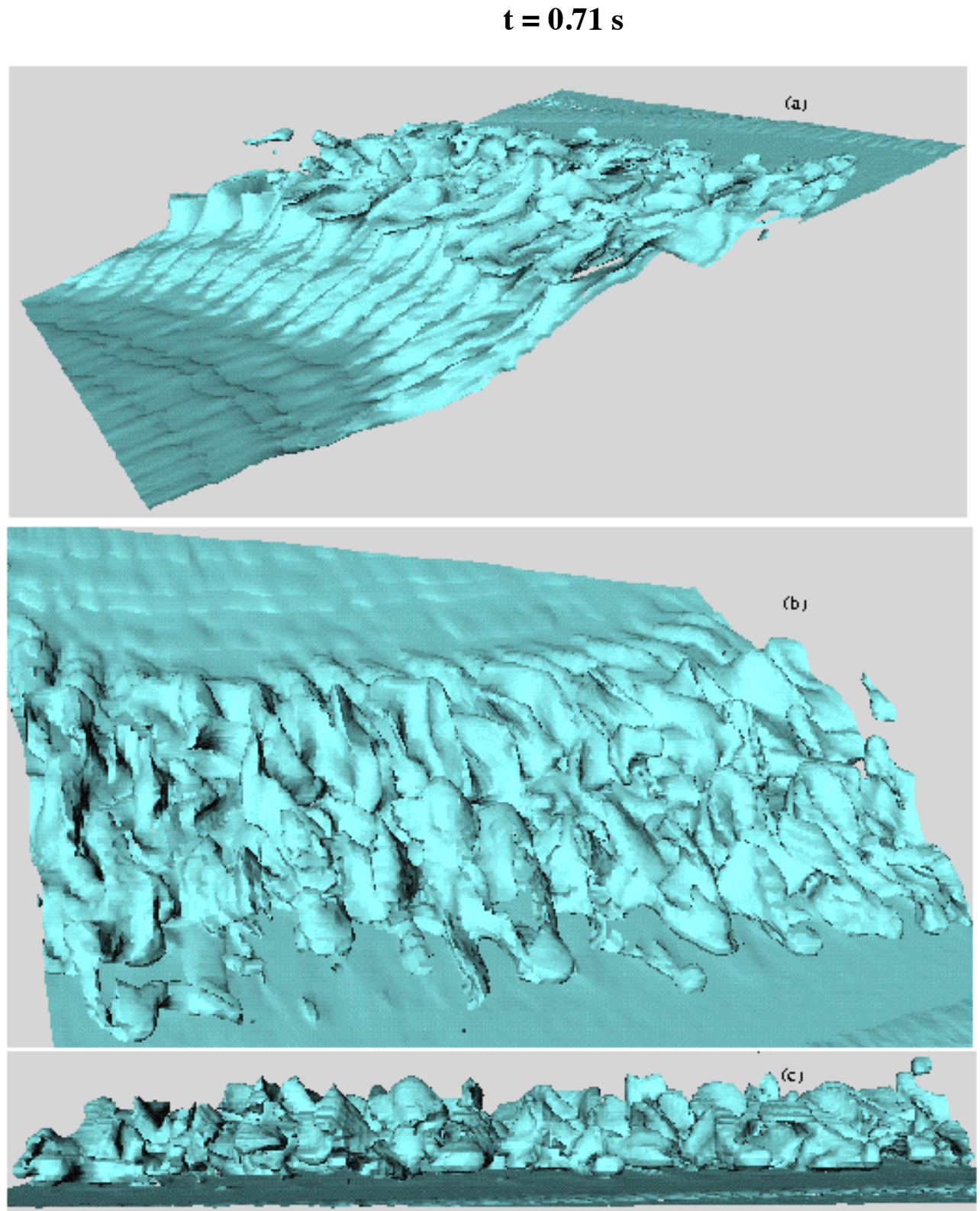

Three-dimensional visualization of the turbulent pad jet and dusty boundary layer flow for Priscilla at $0.711 \mathrm{~s}$ for $+80 \mathrm{~m}<\mathrm{x}<\bar{i}++\mathrm{m}$ of dust concentration of $10 \%$; (a) oblique viewed, (b) viewed looking townd ground zero from atove and (c) viewed looking torisd ground zero at ground level. 


\section{Problem 3. Blast Wave Reflections from Ground Surfaces}

$$
\mathbf{t}=\mathbf{0 . 7 7 8} \mathrm{s}
$$
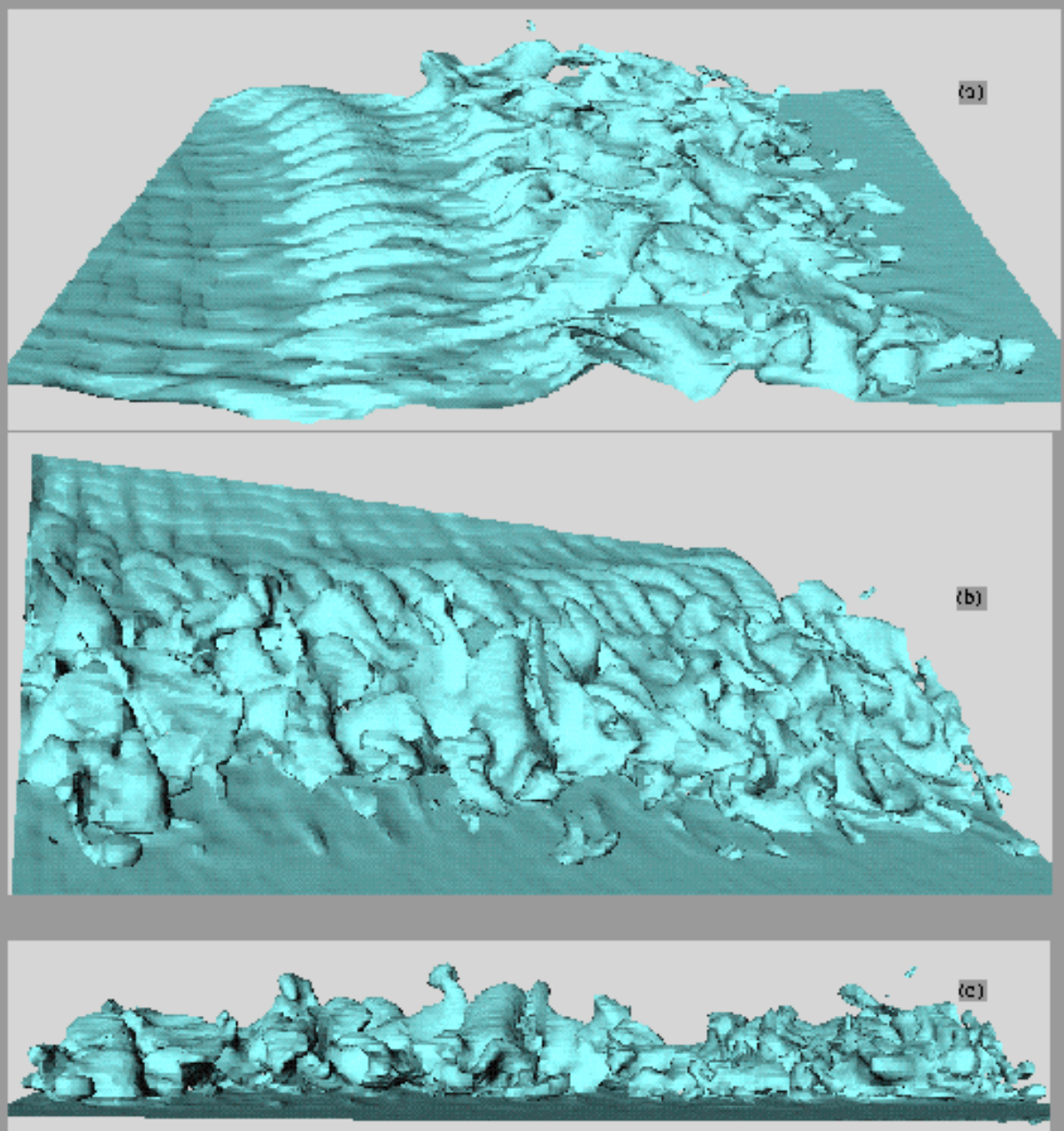

Three-dimensional visulir stion of the inrbolent wall jet and dosty bonndary loyer flow for Priscill st $0.778 s$

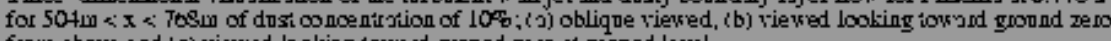
frow above and (c) viewed boking townd grond zero xt groond level. 


\section{Problem 3. Blast Wave Reflections from Ground Surfaces}

$$
\mathbf{t}=\mathbf{0 . 9 4 1 s}
$$
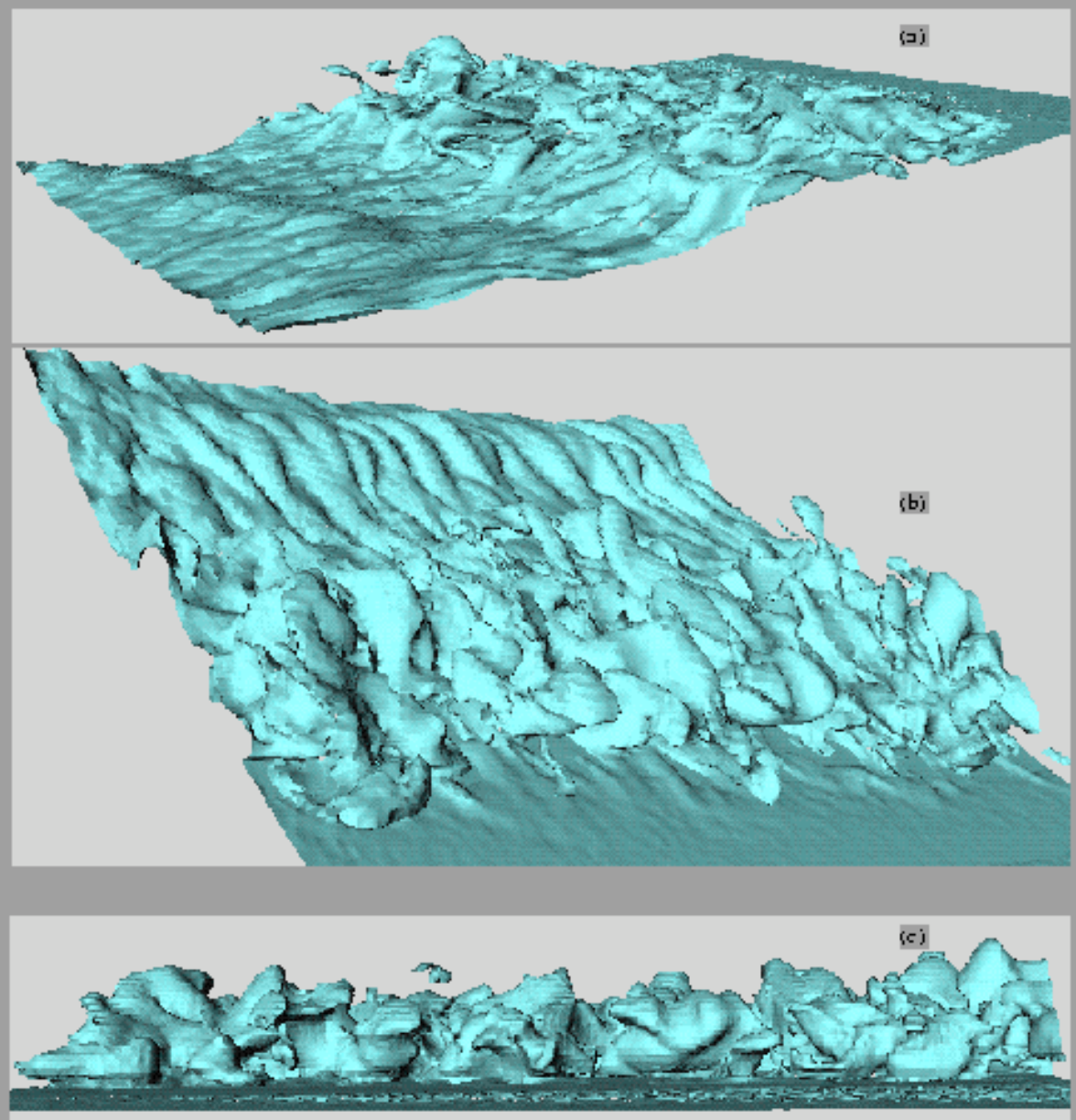

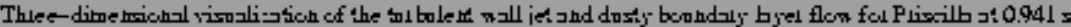

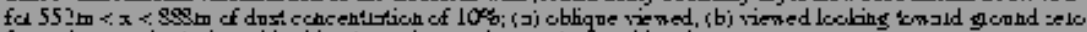

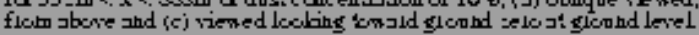




\section{Problem 3. Blast Wave Reflections from Ground Surfaces}
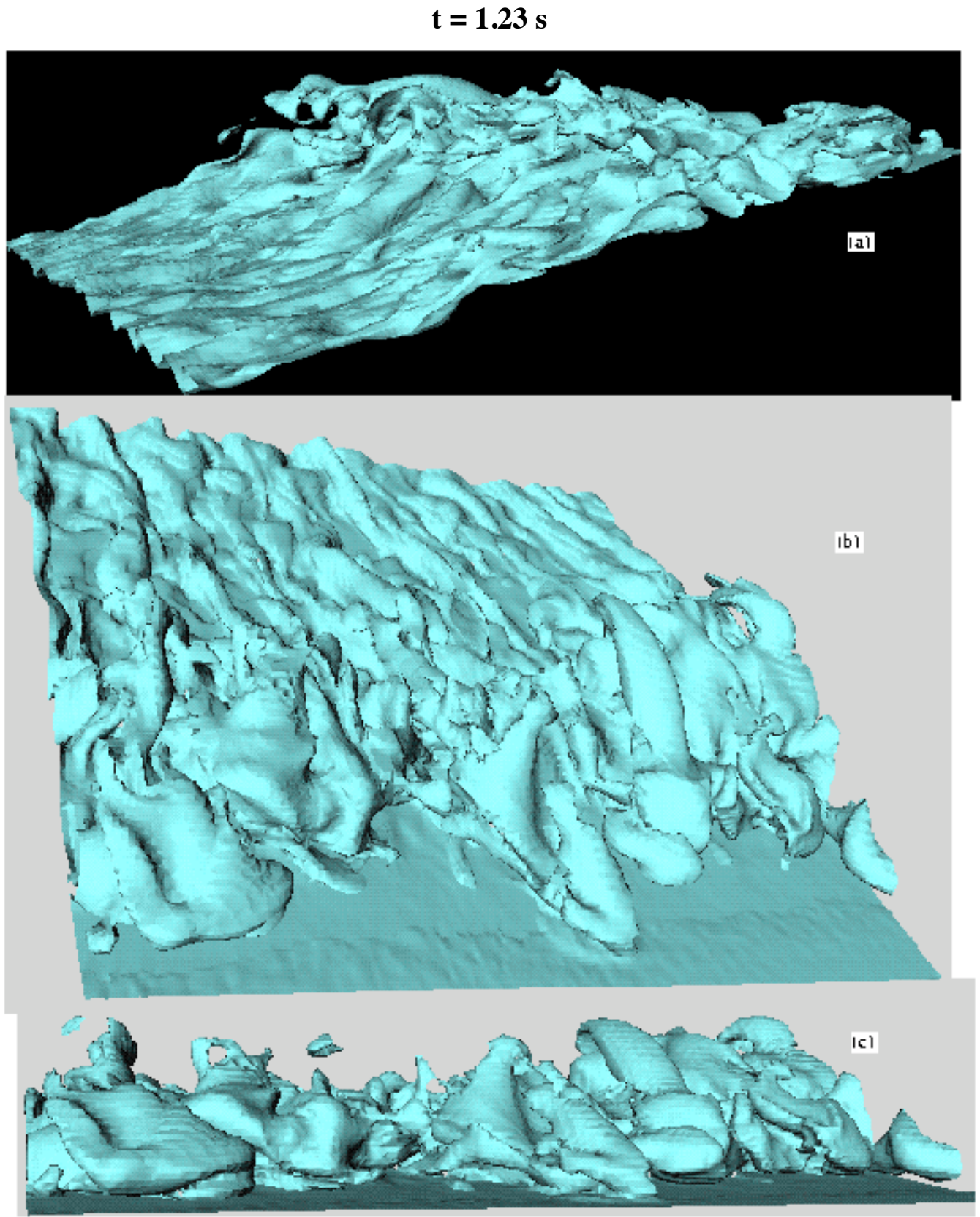

Thee-dimensional visudization of the turbulent wall jet and disty bounday layer flow for Priscilla at $1.23 s$

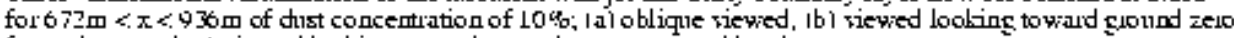
from above and icl riewed lookins towad grom zelo at ground level. 


\section{Problem 3. Blast Wave Reflections from Ground Surfaces}

$$
\mathbf{t}=\mathbf{1 . 3 8} \mathrm{s}
$$

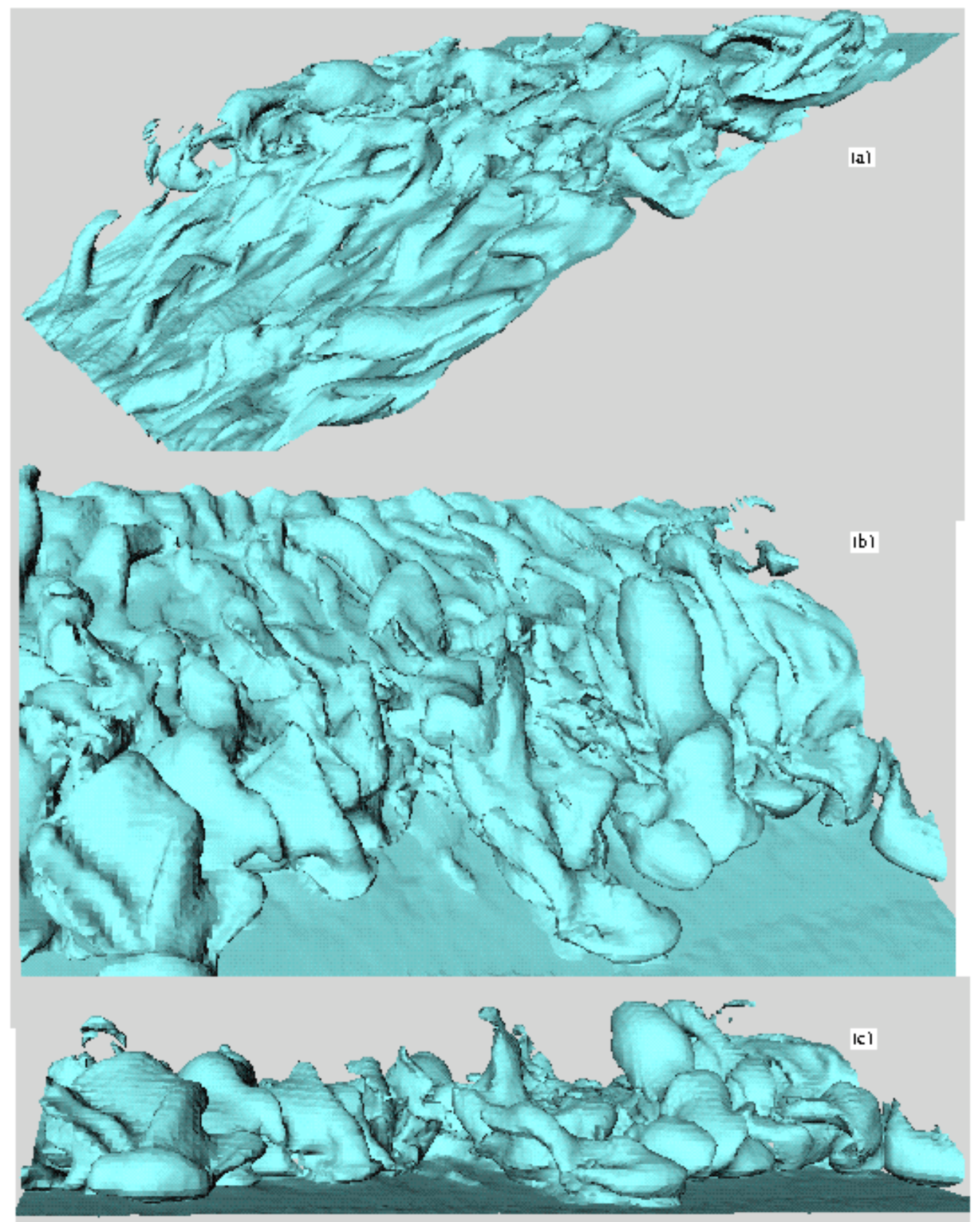

Three-dimensional risualization of the turbulent wall jet and dusty boundary layer flow for Priscilla at $1.38 s$

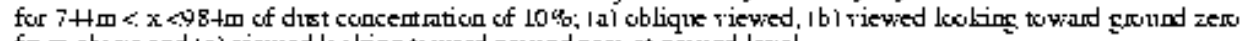
from above and Icl riewed lowbing toward ground zero at gromind level. 


\title{
Problem 4: Spherical Mixing Layers
}

\begin{abstract}
This study explores the properties of spherical combustion clouds in explosions. Two cases are investigated: (i) detonation of a TNT charge and combustion of its detonation products with air, and (ii) shock dispersion of Aluminum powder and its combustion with air. The evolution of the blast wave and ensuing combustion cloud dynamics are studied via numerical simulations with our Adaptive Mesh Refinement (AMR) combustion code. The code solves the multi-phase conservation laws for a dilute heterogeneous continuum as formulated by Nigmatulin. Singlephase combustion (e.g., TNT with air) is modeled in the fast-chemistry limit. Two-phase combustion (e.g., Al powder with air) uses an induction time model based on Arrhenius fits to Boiko's shock tube data, along with an ignition temperature criterion based of fits to Gurevich's data, and an ignition probability model that accounts for multi-particle effects on cloud ignition. Equations of state are based on polynomial fits to thermodynamic calculations with the Cheetah code, assuming frozen reactants and equilibrium products. Adaptive mesh refinement is used to resolve thin reaction zones and capture the energy-bearing scales of turbulence on the computational mesh (ILES approach). Taking advantage of the symmetry of the problem, azimuthal averaging was used to extract the mean and $r m s$ fluctuations from the numerical solution, including: thermodynamic profiles, kinematic profiles, and reaction-zone profiles across the combustion cloud. Fuel consumption was limited to $\sim 60-70 \%$, due to the limited amount of air a spherical combustion cloud can entrain before the turbulent velocity field decays away. Turbulent kinetic energy spectra of the solution were found to have both rotational and dilatational components, due to compressibility effects. The dilatational component was typically about $1 \%$ of the rotational component; both seemed to preserve their spectra as they decayed. Kinetic energy of the blast wave decayed due to the pressure field. Turbulent kinetic energy of the combustion cloud decayed due enstrophy $\overline{\omega^{2}}$ and dilatation $\overline{\Delta^{2}}$.
\end{abstract}

\section{Introduction}

This study investigates combustion clouds embedded in unconfined spherical explosions. Two charge configurations are considered: a 0.5-g spherical PETN booster surrounded by a spherical shell of either 1-g of TNT solid or of 1-g of Aluminum (Al) powder; these provide the fuel for the combustion process. Detonation of booster disperses the fuel, whose expansion drives a blast wave into the surrounding atmosphere. The fuel-air interface is unstable and rapidly evolves into a turbulent mixing layer. The hot detonation products and the shock-heated air serve as ultrastrong ignition sources of the fuel-air mixture, which evolves into a spherical combustion cloud $[1,2]$.

The evolution of the blast wave and ensuing combustion cloud dynamics are studied via numerical simulations with our adaptive mesh refinement (AMR) combustion code $[3,4]$. The code solves the multi-phase conservation laws for a dilute heterogeneous continuum as formulated by Nigmatulin [5]. These equations are integrated with high-order Godunov schemes on uniform Eulerian grid patches. AMR is used to resolve reaction zones and capture energy- 


\section{Problem 4. Spherical Mixing Layers}

bearing scales of the turbulence (the $I L E S^{12}$ approach). This Model of turbulent combustion in explosions has been validated by extensive comparisons with data from calorimeter experiments [1.2]. Computed pressure histories were shown to agree well with measured waveforms in twenty-four different types of experiments [3], [4] - thereby establishing the accuracy of the combustion Model for this class of combustion problem.

However in those previous studies, the nature and details of the computed turbulent fields remained undiagnosed. The purpose of the follow-on work described here was to investigate such turbulent combustion clouds in a geometry whereby the turbulence fields could be easily extracted and investigated. To that end we chose the unconfined spherical mixing layer geometry; due to the point symmetry of the problem, the flow fields could be azimuthally averaged to evaluate the mean and rms profiles of the turbulent fields.

The Model is described in $\S 2$, including: governing conservation laws, multi-phase interaction terms, combustion modelling, equations of state, numerical methods, and statement of the initial value problem. Results are presenting in $\S 3$, including: flow visualization; azimuthal averaging methodology; thermodynamic, kinematic and reaction-zone profiles across the mixing layer; and turbulent kinetic energy spectra. This is followed by a Summary and Conclusions in $\S 4$.

\section{Model}

\subsection{Conservation Laws}

The model is based on the Eulerian multi-phase conservation laws for a dilute heterogeneous continuum as formulated by Nigmatulin [5]. We model the evolution of the gas phase combustion fields in the limit of large Reynolds and Peclet numbers, where effects of molecular diffusion and heat conduction are negligible on the gasdynamic fields. The flow field is governed by the following conservation laws:

Mass: $\quad \partial_{t} \rho+\nabla \cdot(\rho \mathbf{u})=\dot{\sigma}_{s}$

Momentum:

$$
\partial_{t} \rho \mathbf{u}+\nabla \cdot(\rho \mathbf{u u}+p)=\dot{\sigma}_{s} \mathbf{v}-\dot{f}_{s}
$$

Energy:

$$
\partial_{t} \rho E+\nabla \cdot(\rho \mathbf{u} E+p \mathbf{u})=-\dot{q}_{s}+\dot{\sigma}_{s} e_{s}-\dot{f}_{s} \cdot \mathbf{v}
$$

where $\rho, p, u$ represent the gas density, pressure and specific internal energy, $\mathbf{u}$ is the gas velocity vector, and $E \equiv u+\mathbf{u} \cdot \mathbf{u} / 2$ denotes the total energy of the gas phase. Source terms on the right hand side take into account: mass transfer from the particle phase to gas phase $\left(\dot{\sigma}_{s}\right)$, acceleration of particle phase by $\operatorname{drag}\left(\dot{f}_{s}\right)$, and heat exchange $\left(\dot{q}_{s}\right)$ to the particle phase. For gasphase combustion (the TNT-air case), the gasdynamic form of the above equations is used (i.e.,

\footnotetext{
${ }^{12}$ Implicit Large Eddy Simulations (ILES), see reference [32]
} 


\section{Problem 4. Spherical Mixing Layers}

$\dot{\sigma}_{s}=\dot{f}_{s}=\dot{q}_{s}=0$ ). For two-phase combustion, the above equations are supplemented by the following two-phase modeling laws.

We treat the particle phase as an Eulerian continuum field. We consider the dilute limit, devoid of particle-particle interactions, so that the pressure and sound speed of the particle phase are zero. We model the evolution of particle phase mass, momentum and energy fields by the conservation laws of continuum mechanics for heterogeneous media [3],[5]:

Mass:

$$
\partial_{t} \sigma+\nabla \cdot \sigma \mathbf{v}=-\dot{\sigma}_{s}
$$

Momentum:

$$
\partial_{t} \sigma \mathbf{v}+\nabla \cdot \sigma \mathbf{v} \mathbf{v}=-\dot{\sigma}_{s} \mathbf{v}+\dot{f}_{s}
$$

Energy:

$$
\partial_{t} \sigma e_{s}+\nabla \cdot \sigma e_{s} \mathbf{v}=\dot{q}_{s}-\dot{\sigma}_{s} e_{s}
$$

where $\sigma$ and $\mathbf{v}$ represent the particle-phase density and velocity, and $e_{s} \equiv C_{s} T_{s}$ denotes the specific internal energy of the particle phase.

It is important to point out that there is no "heat addition due to combustion" in the energy equation (3). This is a consequence of the absolute energy formulation of the Le Chatalier diagram (Fig. 1), which will be explained in $\$ 2.4$. In this formulation, energy is constant $-\mathrm{a}$ system invariant.

\subsection{Interactions}

The inter-phase interaction terms for mass, momentum, heat and particle burning take the form as described in Khasainov et al. [6]:

Mass Exchange:

$$
\dot{\sigma}_{s}=\left\{\begin{array}{lc}
0 & T_{s}<T_{L} \\
3 \sigma\left(1+0.276 \sqrt{R e_{s}}\right) / t_{s} & T_{s} \geq T_{L}
\end{array}:\right.
$$

Vaporization time: $\quad t_{s}=K d_{s}^{2}$

Momentum Exchange: $\quad \dot{f}_{s}=\frac{3}{4} \frac{\rho}{\rho_{s}} \frac{\sigma}{d_{s}} C_{D}(\mathbf{u}-\mathbf{v})|\mathbf{u}-\mathbf{v}|$

Drag Coefficient:

$$
C_{D}=24 / R e_{s}+4.4 / \sqrt{R e_{s}}+0.42
$$

Heat Exchange:

$$
\dot{q}_{s}=\frac{6 \sigma}{\rho_{s} d_{s}}\left[\frac{N u \lambda\left(T-T_{s}\right)}{d_{s}}+\varepsilon \sigma_{\text {Boltz }}\left(T^{4}-T_{s}^{4}\right)\right]
$$




\section{Problem 4. Spherical Mixing Layers}

where $\operatorname{Re}_{s}=\rho d_{s}|\mathbf{u}-\mathbf{v}| / \mu, N u=2+0.6 \operatorname{Pr} \sqrt{\operatorname{Re}_{s}}, \operatorname{Pr}=C_{p} \mu / k, \rho_{s}=2.7 g / c c$ is the density of the Al particle and $d_{s}$ its diameter, while $K=150 \mathrm{~s} / \mathrm{cm}^{2}$ according to Ingignoli ${ }^{[7]}$. The gas viscosity, specific heat and thermal conductivity are denoted by $\mu, C_{p}$, and $k$, respectively.

Previously, the above interaction terms (7)-(11) where used successfully by Veyssiere and Khasainov [8] to model steady, plane, double-front detonations in gaseous explosive mixtures containing suspended Al particles. In the current implementation, we convert the Al particles to a liquid at $T_{L}=932 \mathrm{~K}$. We assume that once in the liquid phase, the droplets quickly form a micro-mist due to intense accelerations by the gas, as has been demonstrated by Kobiera et al [9] for hexane droplets. At this point the liquid is assumed to be in velocity and temperature equilibrium with the gas phase, and we assume that the micro-mist is susceptible to diffusive combustion (limited by the oxidizer supply rate) without having to reach the boiling temperature of $\mathrm{Al}(\sim 2,300 \mathrm{~K})$.

\subsection{Combustion}

We consider three fuels: PETN detonation products $\left(F_{1}\right)$, TNT detonation products $\left(F_{2}\right)$, and Aluminum $\left(F_{3}\right)$, along with their corresponding combustion products: PETN-air $\left(P_{1}\right)$ and TNTair $\left(P_{2}\right)$ and Al-air $\left(P_{3}\right)$. We model the global combustion of the fuels $F_{k}$ with air $(A)$ producing equilibrium combustion products $P_{k}$ :

$$
F_{k}+A_{k} \Rightarrow P_{k} \quad(k=1,2 \text { or } 3)
$$

The mass fractions $Y_{k}$ of the components are governed by component conservation laws; in the limit of large Peclet numbers (where molecular diffusion effects are negligible) they become:

Fuel-k:

$$
\partial_{t} \rho Y_{F k}+\nabla \cdot \rho Y_{F k} \mathbf{u}=-\dot{s}_{k}+\delta_{k 3} \dot{\sigma}_{s}
$$

Air:

$$
\partial_{t} \rho Y_{A k}+\nabla \cdot \rho Y_{A k} \mathbf{u}=-\sum_{k} \alpha_{k} \dot{s}_{k}
$$

Products-k:

$$
\partial_{t} \rho Y_{P k}+\nabla \cdot \rho Y_{P k} \mathbf{u}=\sum_{k}\left(1+\alpha_{k}\right) \dot{s}_{k}
$$

Fuel and air are consumed in stoichiometric proportions: $\alpha_{k}=A_{k} / F_{k}$. In the above, $\dot{s}_{k}$ represents the global kinetics term. For PETN-air and TNT-air combustion, we assume combustion is mixing limited, so we use the the fast-chemistry approximation: whenever fuel and air enter a computational cell, they are consumed in one time step. For Al-air combustion, we use an induction time model described below. The term $\delta_{k 3} \dot{\sigma}_{s}{ }^{13}$ represents the conversion of $\mathrm{Al}$ from the particle phase to the gas phase, which creates a source of $\mathrm{Al}$ fuel.

\footnotetext{
${ }^{13}$ Kroneker delta: $\delta_{k 3}=1$ if $k=3 \& \delta_{k 3}=0$ if $k \neq 3$
} 


\section{Problem 4. Spherical Mixing Layers}

Following Korobeinikov ${ }^{[10]}$ and Oran ${ }^{[11]}$ for premixed systems, we define an inductiontime fraction: $f(\mathbf{x}, t)$, which is initialized to zero: $f(\mathbf{x}, 0)=0$, and grows to 1 at the end of the ignition delay. It evolves according to the following advection equation:

Induction Model: $\quad \partial_{t} \sigma f+\mathbf{v} \cdot \nabla \sigma f=\sigma / \tau_{i}$

The induction time, $\tau_{\mathrm{i}}$, is based on an Arrhenius fit to Boiko's Al particle data: ${ }^{[12], 13],[14]}$

Induction Time: $\quad \tau_{i}=A e^{E_{0} / R T}$

where $A=1.6 \times 10^{-8} \mathrm{~s}$ and $E_{0}=60 \mathrm{kcal} /$ mole for flake Al. An ignition temperature model $T_{i}$ is also employed:

Ignition Temperature: $T_{i}=T_{m p o}-0.6-\frac{C_{o x}^{0.3} d_{s}}{\lambda} \exp \left(-0.85 \sqrt{d_{s}}\right)$

where $T_{m p o}$ is the melting point of the Aluminum oxide coating $(\sim 2300 \mathrm{~K}), C_{o x}$ denotes the oxidizer concentration, and $\lambda$ represents the thermal conductivity of the gas. It is based on fits to Gurevich's data ${ }^{[15]}$.

An ignition probability model is used to capture multi-particle effects on cloud ignition ${ }^{[14]}$ :

Ignition Probability: $\quad \mu_{c}(\sigma)=\frac{1}{1+\exp \left[\left(\sigma_{0}-\sigma\right) / b\right]}$

Here $\sigma_{0}$ denotes the value of particle concentration resulting in a $50 \%$ probability of cloud ignition, while $b$ represents the slope parameter which determines the width of the probability function. For flake $\mathrm{Al}$ particles ignition in air, they acquire the following values: $\sigma_{0}=130 \mathrm{~g} / \mathrm{m}^{3}$ and $b=20$. Equation (19) was determined empirically by fitting data from the Al particle cloud experiments of Boiko ${ }^{[12]-[14]}$. It models the requirement that the local concentration of particles must be large enough so that ignition of one particle can be passed on to its neighbors - a cloud ignition effect-in contrast to the single-particle ignition effect (e.g., the Gurevich ignition temperature function (18)). Being based on experimental data, it implicitly presumes that the local mixture is within the flammability limits of the Al-air system considered.

Finally, ignition occurs in the model when $f=1$ and $T_{s}>T_{i}$; then the source term becomes:

Ignition: $\quad \dot{s}_{3}=\dot{\sigma} \cdot \mu_{c}(\sigma)$ 


\section{Problem 4. Spherical Mixing Layers}

\subsection{Equations of State}

Our code carries the density and specific internal energy, along with the gas composition in each cell. These are used to calculate the pressure and temperature in a computational cell based on Equations of State (EOS). The thermodynamic states encountered during shock-dispersed-fuel (SDF) explosions have been analyzed in [16]. Here we summarize only the salient features needed for the numerical modeling. The caloric equation of state can be specified in the Le Chatelier plane (Fig. 1) of specific internal energy as a function of temperature: $u(T)$. Notice that these curves are negative at low temperatures; this is because the heat of formation is included in $u(T)$; we call this the absolute energy formulation.

Loci of states covering the temperature range $300 \mathrm{~K} \leq T \leq 6000 \mathrm{~K}$ were calculated by the Cheetah code [17], based on the following thermodynamic assumptions:

- Air: equilibrium isobar ${ }^{14}(p=10 \mathrm{~atm})$

- Aluminum: equilibrium isobar ( $p=10 \mathrm{~atm})$

- PETN detonation products: equilibrium isentrope passing through the Chapman-Jouguet point, with frozen composition for $T<1800 \mathrm{~K}^{15}$

- $\quad$ TNT detonation products: equilibrium isentrope passing through the Chapman-Jouguet point, with frozen composition for $T<1800 \mathrm{~K}^{5}$

- Al-air combustion products: equilibrium $\operatorname{isobar}^{4}(p=10 \mathrm{~atm})$

These loci were fit with piecewise quadratic functions for each component:

$$
u(T)=a_{i} T^{2}+b_{i} T+c_{i} \quad T_{i} \leq T<T_{i+1}
$$

The coefficient values $a_{i}, b_{i}, c_{i}$ are tabulated in [16]. Given the computational cell specific internal energy, $u$, temperature is evaluated by solving the above quadratic for $T$. Pressure is then calculated from the perfect-gas relation ${ }^{16}$ :

$$
p=\rho R T
$$

or from the JWL function in the detonation products gases:

$$
\begin{aligned}
p_{J W L}(v, T)= & A\left(1-\frac{\omega \cdot v_{0}}{R_{1} \cdot v}\right) \exp \left(-R_{1} \cdot v / v_{0}\right)+ \\
& B\left(1-\frac{\omega \cdot v_{0}}{R_{2} \cdot v}\right) \exp \left(-R_{2} \cdot v / v_{0}\right)+R T / v
\end{aligned}
$$

where $v$ is the specific volume $(v=1 / \rho)$. For more details, see [16].

\footnotetext{
${ }^{14}$ We found that the $u(T)_{\text {isobar }}=u(T)_{\text {isochor }}$ for $T<4000 \mathrm{~K}$, hence it was sufficient to fit the internal energy as solely a function of temperature; above $4000 \mathrm{~K}$ this is an approximation, with an error less than $10 \%$.

${ }^{15}$ see Ree et al. [18] for justification

${ }^{16} \mathrm{We}$ found that the perfect-gas law accurately describes constant volume explosions for $p \leq 1 \mathrm{kbar}$ [2].
} 


\section{Problem 4. Spherical Mixing Layers}

\subsection{Numerical Methods}

The above conservation laws were integrated with a high-order generalization of Godunov's method ${ }^{[19]}$. The algorithm is based on an efficient Riemann solver for gasdynamics first developed by Colella and Glaz ${ }^{[20]}$ and Colella and Woodward ${ }^{[21]}$ and extended to generalized conservation laws by Bell et al. ${ }^{[22]}$ and to un-split upwind schemes by Colella ${ }^{[23]}$. The solver was modified to accommodate negative specific internal energies (vid. Fig. 1) associated with our thermodynamic formulation. The algorithm for the particle phase conservation laws is based on a Riemann solver for two-phase flows as developed by Collins et al. [24]. The boundary condition for the particle phase at a solid wall was elastic reflection (i.e., momentum reversal). Source terms are treated with Strang-splitting methods. Since the integrators are based on Riemann solvers; information propagates along characteristics at the correct wave speeds, and they incorporate nonlinear wave interactions within the cell during the time step. They include a limiting step (slope flattening) that automatically reduces the order of approximation in the neighborhood of discontinuities, while in smooth regions of the flow the scheme is second order in time and space.

The Godunov algorithm forms the integrator for our adaptive mesh refinement (AMR) code. Our adaptive methods are based on the block-structured AMR algorithms of Berger \& Colella ${ }^{[25]}$, and extended to three-dimensional hyperbolic systems by Bell et al. ${ }^{[26]}$. Embedded boundary methods ${ }^{[27]}$ are used to represent irregular geometries. In this approach, regions to be refined are organized into rectangular patches, with several hundred to several thousand gridpoints per patch. One can refine on discontinuities (shocks and contact surfaces), on Richardson error estimates, or for present purposes, on reaction zones. Grid patches are assigned to processors by a knapsack algorithm based on workload estimates, ${ }^{[28]}$ so the AMR code runs efficiently on massively-parallel computers. ${ }^{[29]}$

AMR is also used to refine turbulent mixing regions; by successive refinements we are able to capture the energy-bearing scales of the turbulence on the computational grid. In this way we are able to compute the effects of turbulent mixing without resorting to explicit turbulence modeling. This is consistent with the so-called MILES (Monotone Integrated Large-Eddy Simulation) approach of Boris ${ }^{[30],[31]}$. A comprehensive review of such Implicit Large-Eddy Simulation (ILES) methods may be found in Grinstein et al. ${ }^{[32]}$. Verification of the ability of our upwind scheme to produce velocity fields that replicate the Kolmogorov spectrum of turbulent flow, has been demonstrated by Aspden et al. ${ }^{[33]}$ for incompressible flows.

\subsection{Initial Conditions}

We simulated two cases of combustion in unconfined explosions: afterburning of TNT in air and combustion of Al powder with air. These fuels were dispersed by the detonation of a PETN booster. Charge configurations were:

- $\quad 0.5$-g spherical PETN booster $\left(\rho_{0}=1 \mathrm{~g} / c c\right)$, surrounded by 1.0 -g spherical shell of TNT fuel $\left(\rho_{0}=1 \mathrm{~g} / c c\right)$, 


\section{Problem 4. Spherical Mixing Layers}

- 0.5-g spherical PETN booster $\left(\rho_{0}=1 \mathrm{~g} / \mathrm{cc}\right.$ ), surrounded by 1.0 -g spherical shell of loose Al powder $\left(\rho_{0}=0.6 \mathrm{~g} / \mathrm{cc}\right){ }^{17}$

The computational domain was: $-100 \mathrm{~cm}<x<100 \mathrm{~cm} ;-100 \mathrm{~cm}<y<100 \mathrm{~cm} ;-100 \mathrm{~cm}<$ $z<100 \mathrm{~cm}$. A base-grid of $\Delta_{0}=3 \mathrm{~mm}$ was used, with four levels of refinement, so the mesh size of the finest grid was $\Delta_{4}=0.2 \mathrm{~mm}$. The domain was initialized with air at: $p_{a}=1 \mathrm{~atm}$, $T_{a}=273 \mathrm{~K}, \rho_{a}=1.2 \mathrm{mg} / \mathrm{cc}$. The simulation was run to a time of $4 \mathrm{~ms}$.

\section{Results}

\subsection{Flow Visualization}

Turbulent combustion inside the cloud is visualized by a color-bar representation of the temperature field in Fig. 2. The TNT-air cloud reaches a combustion temperature of $\sim 2000 \mathrm{~K}$, while the Al-air cloud reaches a combustion temperature between 3000 and $4000 \mathrm{~K}$. For the TNT case, combustion occurs along thin flame sheets (Fig. 3), while a distributed-combustion mode is realized for the Al-powder case (Fig. 2 d-f) due to two-phase flow effects. By performing reactive and non-reactive flow simulations shown in Fig. 4, it was demonstrated that combustion caused no change in the pressure field during the positive phase of the blast wave-in other words, combustion in the fireball is isobaric. This is confirmed by pressure histories measured in a 40 liter calorimeter (Fig. 5); experiments in nitrogen versus air illustrate that combustion effects become noticeable after reflections from the chamber walls.

\subsection{Azimuthal Averaging}

The problem under consideration is point symmetric. In the absence of instabilities at the fuel-air interface, the solution is solely a function of radius and time: $\Phi(r, t)$. An example of this is blast wave from a TNT charge as calculated by Brode ${ }^{[34],[35]}$. One can obtain the radial solution by azimuthally averaging the computed 3D flow field to produce $\overline{\Phi(r, t)}$. Given that mean, one can compute second moments of the flow field to evaluate root-mean-squared ( $r m s$ ) fluctuations about the mean: $\Phi^{\prime}(r, t)_{r m s}$. The numerical algorithm for accomplishing this is described in Appendix A. This technique was first used by Kuhl ${ }^{[36]}$ to investigate spherical mixing layers in TNT explosions.

\subsection{Thermodynamic Profiles}

The azimuthally averaged thermodynamic profiles of density, temperature and pressure in the TNT combustion cloud are presented in Fig. 6. The mean fields (left column) depict the onedimensional blast wave fields engendered by the detonation of the TNT charge (vid. Brode ${ }^{[34][35]}$ ). The rms fields quantify the consequences of the three-dimensional turbulent mixing. Since

\footnotetext{
${ }^{17}$ This $\mathrm{Al}$ powder density corresponds to a porosity of $80 \%$ (i.e. a volume fraction of $20 \%$ ); it was a consequence of the inefficient packing density of the flake $\mathrm{Al}$ particles used in the experiments. It is also why the dilute heterogeneous continuum model used here is successful.
} 


\section{Problem 4. Spherical Mixing Layers}

pressure fluctuations are very small ( $\sim 1 \%$ of ambient), density fluctuations track temperature fluctuations, which increase in time due to combustion.

\subsection{Kinematic Profiles}

Azimuthally averaged kinematic profiles in the TNT combustion cloud are presented in Figs. 7. The mean radial velocity profiles again resemble those from TNT charges. ${ }^{[34],[35]}$ The rms velocity-fluctuation profiles $\left(u_{r}^{\prime}, u_{\theta}^{\prime}, u_{\phi}^{\prime}\right)$ are similar in magnitude; at $0.2 \mathrm{~ms}$ they peak at $\sim 60 \mathrm{~m} / \mathrm{s}$ and decay to $\sim 5 \mathrm{~m} / \mathrm{s}$ by $4 \mathrm{~ms}$. Thus the turbulence is isotropic in this problem. The Reynolds stress profiles at $t=0.65 \mathrm{~ms}$ are depicted in Fig. 7e. This flow is dominated by the diagonal stresses $\left(\tau_{r r}, \tau_{\theta \theta}, \tau_{\phi \phi}\right)$ whose profiles are similar, while the cross terms $\left(\tau_{r \theta}, \tau_{r \phi}, \tau_{\theta \phi}\right)$ are negligible. Enstrophy: $\omega^{2}$ (or inner product of the vorticity vector: $\omega$ ), provides scalar measure of the vorticity field. Its mean ${ }^{18}$ profiles $\overline{\omega\left(R_{n}, t\right)^{2}}$ are presented in Fig. 7f; they decay by two orders of magnitude during the $4 \mathrm{~ms}$ of the simulation.

\subsection{Reaction Zone Profiles}

Development of the component mass-fraction profiles: $Y_{k}(r, t)$ (where $k=D, D P, F, C P, A$ representing PETN driver gas, PETN-air combustion products, TNT detonation products as a fuel, TNT-air combustion products and air, respectively) are presented in Fig. 8. Most prominently Fig. 8 shows the initiation and growth of the combustion products CP near $r=10$ $\mathrm{cm}$, and the depletion of fuel: $\mathrm{F}$ and air: A due to combustion. Eventually the combustion products spread throughout the cloud (Fig. 8f) as a consequence of turbulent mixing. Development of the component mass-fraction profiles in the Al-air combustion cloud, are presented in Fig. 9. Profiles are qualitatively similar to those of the TNT-air cloud (Fig. 8).

Details of the early time growth and evolution of the combustion Products mass profiles for the TNT case are presented in Fig. 10. The reaction zone starts at $10 \mu \mathrm{s}$ near $r \sim 3 \mathrm{~cm}$ (Fig. 10c); by $100 \mu s$ its center has migrated out to $r \sim 10 \mathrm{~cm}$ and has broadened into a shell of width $\sim 6 \mathrm{~cm}$. By $4 \mathrm{~ms}$, it has moved out to $\mathrm{r} \sim 15 \mathrm{~cm}$; Products have spread throughout the cloud due to turbulent mixing. rms fluctuations of Products were approximately 50\% the mean (Fig. 10d).

It is worth recalling that the simulations were performed for 1-g fuel charges. Although not labeled as such in Figs. 8-10, one can consider the radial dimensions and times as $\mathrm{r}\left(\mathrm{cm} / \mathrm{g}^{1 / 3}\right)$ and $t\left(m s / g^{1 / 3}\right)$ when scaling to other charge masses.

\subsection{Fuel Consumption}

Fuel histories are depicted in Fig. 11. In the present unconfined cases, 60-70\% of the fuel had been consumed by $4 \mathrm{~ms}$, and the slope of the fuel curves $m(t)$ was becoming asymptotically small. In the previous calorimeter calculations, virtually all of the fuel had been consumed by 4

\footnotetext{
${ }^{18}$ Mean enstrophy is computed from: $\overline{\omega\left(R_{n}, t\right)^{2}} \equiv \frac{1}{\delta V} \iiint \omega\left(R_{n}, \theta, \phi\right) \cdot \omega\left(R_{n}, \theta, \phi\right) d V$ according to A3
} 


\section{Problem 4. Spherical Mixing Layers}

ms - due to the continued stirring induced by shock reverberations in the chamber. This comparison suggests that there is a limit to how much air the unconfined spherical mixing layer can entrain before the turbulent velocity field dies (see Figs. 7b, c, d). For the stoichiometric requirements of the fuels considered $\left(3.5 \leq \alpha_{s} \leq 4\right)$, the mixing limitation has the effect of limiting fuel consumption to $60-70 \%$.

\subsection{Turbulent Kinetic Energy}

We have analyzed the evolution of the turbulent velocity field in the combustion cloud. We start by defining the fluctuation velocity as a fluctuation about the mean radial velocity:

$$
\mathbf{u}^{\prime}(\mathbf{x}, t) \equiv \mathbf{u}(\mathbf{x}, t)-\overline{u_{r}(r, t)}
$$

where the mean radial velocity $\overline{u_{r}(r, t)}$ was evaluated by the azimuthal-averaging procedure. Since the flow is compressible, we decompose the fluctuation velocity into rotational and dilatational components (see Batchelor ${ }^{[37]}$ ):

$$
\mathbf{u}^{\prime}(\mathbf{x}, t)=\mathbf{u}_{\omega}^{\prime}(\mathbf{x}, t)+\mathbf{u}_{\Delta}^{\prime}(\mathbf{x}, t)
$$

The rotational component depends on the vorticity field according to the Biot-Savart law:

$$
\mathbf{u}_{\omega}^{\prime}(\mathbf{x}, t)=\frac{-1}{4 \pi} \int \frac{\mathbf{s}}{S} \times \omega^{\prime}(x, t) d V(x)
$$

where the vorticity is derived from the fluctuation velocity according to $\omega^{\prime} \equiv \nabla \times \mathbf{u}^{\prime}$, and $\mathbf{S}=\mathbf{x}-x$ and $s=|\mathbf{s}|$. The rotational component is solenoidal: $\nabla \cdot \mathbf{u}_{\omega}^{\prime}=0$. Similarly, the dilatational component depends on the dilatation field according to its Biot-Savart law:

$$
\mathbf{u}_{\Delta}^{\prime}(\mathbf{x}, t)=\frac{1}{4 \pi} \int \frac{\mathbf{s}}{S} \Delta^{\prime}(x, t) d V(x)
$$

where the dilatation is derived from the fluctuation velocity according to $\Delta^{\prime}=\nabla \cdot \mathbf{u}_{\Delta}^{\prime}$. The dilatational component is irrotational: $\nabla \times \mathbf{u}_{\Delta}^{\prime}=0$. Then one can define the fluctuating kinetic energy components:

$$
F K E_{\omega}=\frac{1}{2}\left(\mathbf{u}_{\omega}^{\prime}\right)^{2} \quad \text { and } \quad F K E_{\Delta}=\frac{1}{2}\left(\mathbf{u}_{\Delta}^{\prime}\right)^{2}
$$

Based on the above decomposition, the Fourier transforms of $F K E_{\omega}(\mathbf{x}, t)$ and $F K E_{\Delta}(\mathbf{x}, t)$ were computed at different times. Results are plotted as the turbulent kinetic spectrum in Fig. 12. Initially, all the kinetic energy resides in radial velocity field: $\overline{u_{r}}$ of the blast wave (i.e., $\mathbf{u}^{\prime}=0$ ). By $50 \mu s$ (Fig. 2a), fluctuations have started to grow on the TNT-air interface; they begin splitting into rotational and dilatational components (Fig. 12a) - with the dilatational 


\section{Problem 4. Spherical Mixing Layers}

component being dominant. By $100 \mu \mathrm{s}$, the fluctuating kinetic energy has concentrated in the rotational component; thereafter the dilatational component is about $1 \%$ of the rotational component. Both seem to preserve their spectrum as they decay (Fig. 12b). These results are reminiscent of the behavior of the solenoidal and compressional spectra predicted by Porter et al [38], [39] who performed gasdynamic simulations of decaying turbulence in a supersonic field, based on the PPM Godunov algorithm ${ }^{[24]}$ (a relative of the Godunov scheme used in these simulations). For fundamental discussions of turbulence spectra see Kolmogorov ${ }^{[40]}$ and the monograph by Zarkov, L'vov and Falkovich ${ }^{[4]]}$.

Consider the global kinetic energy equation, as derived from the compressible NavierStokes equation (derivation may be found in Appendix B):

$$
\frac{d}{d t} \overline{\rho K E}=-\overline{\mathbf{u} \cdot \nabla p}-\mu \overline{\omega^{2}}-\frac{4}{3} \mu \overline{\Delta^{2}} \quad(t<2 m s)
$$

where $\mu$ represents the shear viscosity coefficient. From this one can see that the global kinetic energy decays because of the pressure field, the enstrophy: $\overline{\omega^{2}}$ and the dilatation: $\overline{\Delta^{2}}$. This relation becomes invalid when the shock wave runs off the grid (boundary integral terms would appear in eq. (29)); this happens at $\sim 2 m s$ in this simulation.

Evolution of the global kinetic energy: $K E(t)$, fluctuating kinetic energy: $F K E(t)$ and enstrophy: $\overline{\omega^{2}}$ are presented in Fig. 13. Decay of the global kinetic energy is caused by the above noted mechanisms. The steep decay at $2 \mathrm{~ms}$ signals that the blast wave has exited the computational domain. The decay of the fluctuating kinetic energy is related to the energy cascade of the turbulent flow. It is explained qualitatively by second and third terms on the righthand side of eq. (29), where in our simulation, $\mu$ would represent an effective numerical viscosity induced by the truncation terms in the Godunov algorithm.

\section{Summary and Conclusions}

1. Combustion occurs in thin flame sheets for the gaseous (TNT) combustion cloud, while a distributed mode of combustion is observed in the two-phase (Al) combustion cloud.

2. Combustion is isobaric in these non-premixed systems.

3. The spherical cloud geometry is ideal for studying turbulence properties of combustion in explosions. By using azimuthal averaging, one can extract all turbulence properties (mean profiles, rms profiles, turbulence spectra, etc.) from a single numerical simulation. In the present simulations the ensemble size was $10^{5}$ samples per radial position; because of this large size, the profiles are very smooth.

4. The so-evaluated Reynolds stress profiles were found to be dominated by the diagonal components - thereby indicating that turbulence is isotropic in spherical mixing layers. 


\section{Problem 4. Spherical Mixing Layers}

5. Azimuthal averaging was also used to evaluate the evolution of the combustion zone in the cloud. Combustion started at the fuel-air interface and spread throughout the cloud due to the turbulent velocity field.

6. Due to compressibility effects, the velocity field contained both rotational and dilatational components.

7. Initially all the kinetic energy resided in the radial (dilatation) velocity component. By $50 \mu s$, the fuel-air interface started to become unstable, and velocity fluctuations appeared. At that time, most of the fluctuating kinetic energy resided in the dilatational velocity field. By $100 \mu s$, the fluctuating kinetic energy had evolved into the rotational velocity field; thereafter, the dilational kinetic energy was about $1 \%$ of the rotational kinetic energy.

8. Kinetic energy of the blast wave decayed due to the pressure field.

9. The fluctuating (turbulent) kinetic energy decayed because of the enstrophy: $\overline{\omega^{2}}$ and dilatation: $\overline{\Delta^{2}}$ fields.

10. The shape of the turbulence spectrum depends on the turbulent cascade process. In these simulations we use an implicit large eddy simulation approach in which dissipation at the fine scales is provided by the numerical dissipation in the second-order Godunov algorithm (see Aspden et al. [33] for details).

\section{Appendix A: Azimuthal Averaging}

We take advantage of the point symmetry inherent in spherical blast waves and azimuthally average the flow field to extract the mean and rms profiles from the numerical solution. Recall that the flow field $\Phi(x, y, z, t)$ is computed and stored at cell-centered points $P(x, y, z)$ on a Eulerian grid. The points are transformed to points $P(r, \theta, \phi)$ on a corresponding spherical grid, by the trigonometric formulas:

$$
|\mathbf{r}|=\sqrt{x^{2}+y^{2}+z^{2}} \& \theta=\arccos (z /|\mathbf{r}|) \& \phi=\arctan (x / y)
$$

We consider spherical shell volume $\delta V$ at radius $R_{n}$

$$
\delta V=\left[\iint\left(R_{n} d \theta\right)\left(R_{n} \sin \theta d \phi\right)\right] \delta r=4 \pi R_{n}^{2} \delta r
$$

A shell thickness equal to the mesh size is assumed $(\delta r=\Delta)$. We denote computational cells located within this shell volume by $P_{n}\left(R_{n}, \theta, \phi\right)$ and the corresponding flow field values by $\Phi_{n}\left(R_{n}, \theta, \phi, t\right)$. We then average the flow field at fixed $R_{n}$ to evaluate the mean field:

Mean:

$$
\overline{\Phi\left(R_{n}, t\right)}=\frac{1}{\delta V\left(R_{n}\right)} \iiint \Phi\left(R_{n}, \theta, \phi, t\right) d V=\frac{1}{N} \sum^{N} \Phi_{n}
$$




\section{Problem 4. Spherical Mixing Layers}

The ensemble size: $N=4 \pi\left(R_{n} / \Delta\right)^{2}$, is approximately $10^{5}$, except near the origin (see Table A1). Given the mean, one can then compute fluctuations about the mean:

$$
\overline{\Phi^{\prime}\left(R_{n}, t\right)^{2}}=\frac{1}{\delta V\left(R_{n}\right)} \iiint\left[\Phi\left(R_{n}, t\right)-\overline{\Phi\left(R_{n}, t\right)}\right]^{2} d V=\frac{1}{N} \sum^{N}\left[\Phi_{n}-\Phi\left(R_{n}, t\right)\right]^{2}
$$

and root-mean-squared (rms) fluctuations

$r m s: \quad \Phi^{\prime}\left(R_{n}, t\right)_{r m s}=\sqrt{\overline{\Phi^{\prime}\left(R_{n}, t\right)^{2}}}$

These were used to construct the evolution of the mean and rms profiles of the combustion cloud. We note in passing that this azimuthal-averaging technique was first used by Kuhl [36] to analyze spherical mixing layers in TNT explosions.

Table A1. Ensemble size versus radius $\left(\Delta_{2}=0.8 \mathrm{~mm}\right)$

\begin{tabular}{|c|c|}
\hline$R_{n}(\mathrm{~cm})$ & $N=4 \pi\left(R_{n} / \Delta\right)^{2}$ \\
\hline 1 & 2000 \\
\hline 5 & $0.5 \times 10^{5}$ \\
\hline 10 & $2 \times 10^{5}$ \\
\hline 15 & $4.6 \times 10^{5}$ \\
\hline 20 & $8 \times 10^{5}$ \\
\hline
\end{tabular}

\section{Appendix B: Global Kinetic Energy Equation for Viscous Compressible Flow}

The compressible Navier-Stokes equations may be written in the form:

$$
\partial_{t} \rho \mathbf{u}+\nabla \rho \mathbf{u u}=-\nabla p+\mu \nabla^{2} \mathbf{u}+\frac{1}{3} \mu \nabla(\Delta)
$$

where $\Delta \equiv \nabla \cdot \mathbf{u}=\operatorname{div} \mathbf{u}, \nabla^{2} \mathbf{u}=\operatorname{div}(\operatorname{grad}(\mathbf{u})), \mu$ represents the coefficient of viscosity and assumes the Stokes hypothesis. Taking the inner product with $\mathbf{u}$ gives the compressible kinetic energy equation:

$$
\partial_{t} \rho K E+\nabla \rho K E \mathbf{u}=-\mathbf{u} \cdot \nabla p+\mu \mathbf{u} \cdot \nabla^{2} \mathbf{u}+\frac{1}{3} \mu \mathbf{u} \cdot \nabla(\Delta)
$$

where $K E=\mathbf{u} \cdot \mathbf{u} / 2$. Using vector calculus relations, one finds 


\section{Problem 4. Spherical Mixing Layers}

dilatation function: $\quad \mathbf{u} \cdot \nabla(\Delta)=-\Delta^{2}+\nabla \cdot(\mathbf{u} \Delta)$

dissipation function: $\quad \mathbf{u} \cdot \nabla^{2} \mathbf{u}=-\omega^{2}-\Delta^{2}+\nabla \cdot(\mathbf{u} \Delta)+\nabla \cdot(\mathbf{u} \times \omega)$

Inserting these into (B2), one finds the kinetic energy equation for compressible viscous flow:

$$
\begin{aligned}
\partial_{t} \rho K E+\nabla \cdot \rho K E \mathbf{u}= & -\mathbf{u} \cdot \nabla p-\mu \omega^{2}-\frac{4}{3} \mu \Delta^{2}+ \\
& \mu\left\{\nabla \cdot(\mathbf{u} \times \omega)+\frac{4}{3} \nabla \cdot(\mathbf{u} \Delta)\right\}
\end{aligned}
$$

Integrating over a spherical volume $V(R)$ whose radius: $R$ is greater than the spherical shock radius: $R_{s}(t)$, all divergence terms are zero. Thus we find the evolution equation for the global (volume-averaged) kinetic energy:

$$
\frac{d}{d t} \overline{\rho K E}=-\overline{\mathbf{u} \cdot \nabla p}-\mu \overline{\omega^{2}}-\frac{4}{3} \mu \overline{\Delta^{2}}
$$

Decompose the kinetic energy, based on the mean and fluctuating velocity components $u_{r}$ and $\mathbf{u}^{\prime}$ :

$$
K E \equiv \frac{1}{2} \mathbf{u} \cdot \mathbf{u}=\frac{1}{2} \overline{u_{r}}+\overline{u_{r}} \mathbf{u}^{\prime}+\frac{1}{2} \mathbf{u}^{\prime} \cdot \mathbf{u}^{\prime}
$$

Integrating over the same control volume $V$ gives:

$$
\overline{\rho K E}=\frac{1}{V} \int \frac{1}{2} \rho u_{r}^{2} d V+\frac{1}{V} \int \frac{1}{2} \rho \mathbf{u}^{\prime} \cdot \mathbf{u}^{\prime} d V=\overline{\rho K E}_{B W}+\overline{\rho F K E}
$$

Decompose the fluctuating kinetic energy into rotational and dilatational components:

$$
\overline{\rho F K E}=\frac{1}{V} \int \frac{1}{2} \rho \mathbf{u}^{\prime} \cdot \mathbf{u}^{\prime} d V=\overline{\rho K E}_{\omega}+\overline{\rho K E}_{\Delta}
$$

where $\overline{\rho K E}_{\omega}=\frac{1}{V} \int \frac{1}{2} \rho \mathbf{u}_{\omega}^{\prime} \cdot \mathbf{u}_{\omega}^{\prime} d V$ and $\overline{\rho K E}_{\Delta}=\frac{1}{V} \int \frac{1}{2} \rho \mathbf{u}_{\Delta}^{\prime} \cdot \mathbf{u}_{\Delta}^{\prime} d V$. Thus the global kinetic energy equation for compressible viscous flow may be written as:

${ }^{19}$ Since $\int \overline{u_{r}} \mathbf{u}^{\prime} d V=0$

${ }^{20}$ By orthogonality: $\int \mathbf{u}_{\omega}{ }_{\omega} \cdot \mathbf{u}_{\Delta}{ }_{\Delta} d V=0$ (see eqs. $26 \& 27$ ) 


\section{Problem 4. Spherical Mixing Layers}

$$
\frac{d}{d t} \overline{\rho K E}_{B W}+\frac{d}{d t}{\overline{\rho K E_{\omega}}}_{d t}+\frac{d}{\rho K E_{\Delta}}=-\overline{\mathbf{u} \cdot \nabla p}-\mu \overline{\omega^{2}}-\frac{4}{3} \mu \overline{\Delta^{2}}
$$

This provides insight into the decay of turbulent kinetic energy in compressible viscous flows.

\section{References}

[1] A. L. Kuhl, H. Reichenbach, Combustion effects in confined explosions, Proc. Combustion Institute 32 (2), 2009, pp. 2291-2298.

[2] A. L. Kuhl, H. Reichenbach, Barometric calorimeters, Combustion, Explosions and Shock Waves, 4 (2) 2010 pp. 271-278 (also in Khimicheskaya Fizika 4(2) 2010 pp. 271-278).

[3] A. L. Kuhl, J. B. Bell, V. E. Beckner, Heterogeneous continuum model of aluminum particle combustion in explosions, Combustion Explosion and Shock Waves 46 (4), pp. 433-448, 2010 (also in Физика горения и взрыва, 2010, т. 46, $\mathrm{N}^{\mathrm{o}}$ 4).

[4] A. L. Kuhl, J. B. Bell, V. E. Beckner, H. Reichenbach, Gasdynamic model of turbulent combustion in TNT explosions, Proc. Combustion Institute 33 (2), 2011, pp. 2177-2185.

[5] Nigmatulin, R. I., Dynamics of Multi-phase Flows, Vol. 1. Moscow, Nauka, 1987, 464 pp.

[6] Khasainov, B., Kuhl, A. L., Victorov, S., Neuwald, P. Model of Non-Premixed Combustion of Al-Air Mixtures, $14^{\text {th }}$ APS Meeting on Shock Compression Condensed in Matter, AIP Proceedings 845, part 1, 2005, pp. 449-452.

[7] Ingignoli, W., Etude de la formation et de la propagation des détonations dans des suspensions de particules d'aluminium en atmosphère oxydante ou réactive. Thèse d’Université de Poitiers, France, 1999. 495 pp.

[8] Veyssiere, B., and Khasainov, B. A Model for steady, Plane, Double-front Detonations (DFD) in Gaseous Explosive Mixtures with Aluminum Particles in Suspension, Combustion and Flame, Vol. 85 (1, 2), 1991, pp. 241-253.

[9] Kobiera, A., Szymczyk, J., Wolanski, P. and Kuhl, A. L., Study of the Shock-Induced Acceleration of Hexane Droplets, Shock Waves, Vol. 18 (6), 2009, pp. 475.

[10] Korobeinikov, V. P., Levin, V. A., Markov, V. V. and Chernyi, G. G., Propagation of blast waves in a combustible gas, Astonautic Acta, Vol 17, pp. 529-537, 1972.

[11] Oran, E. S., Boris, J. P., Young, T. R., Flanigan, M. and Picone, M., Simulation of gas phase detonations: introduction of an induction parameter model, NRL Report 4255, 1980.

[12] Boiko, V. M., Lotov, V. V. and Papyrin, A. N., Ignition of Gas Suspensions of Metallic Powders in Reflected Shock Waves, Combustion, Explosion and Shock Waves, Vol. 25, No. 2, pp. 193-199, 1989 [Fizika goreniya I Vzryva, Vol. 25 No. 2, pp. 67-74, 1989].

[13] Boiko, V. M. and Poplavski, S. V., Self-ignition and ignition of Al powders in shock waves, Shock Waves, Vol. 11, pp. 289-295, 2002.

[14] A. L. Kuhl, V. M. Boiko, Ignition of Aluminum particles and clouds, $41^{\text {st }}$ ICT Conference, Energetic Materials: High Performance, Insensitive Munitions and Zero Pollution, Fraunhofer Institut für Chemisch Technologie ICT, 2010, pp. 29.1-29.11.

[15] M. A. Gurevich, K. I Lapinka, E. S Ozerov, Ignition Limits of Aluminum Particles, Fizika Goreniya i Vzryva, Vol. 6, No. 2, pp. 172-175, 1970.

[16] Kuhl, A. L., Khasainov, B., Quadratic Model of Thermodynamic States in SDF Explosions, Energetic Materials: Characterization and Performance of Advanced Systems, Thirty-eighth Int. Annual Conference of the Institut Chemische Technologie (ICT), 2007, pp. 143.1-11.

[17] L. E. Fried, CHEETAH 1.22 User's Manual, Report No. UCRL-MA-117541, LLNL (1995). 


\section{Problem 4. Spherical Mixing Layers}

[18] F. H. Ree, W. J. Pitz, M. van Thiel, P. C. Souers, "Over-abundance of Carbon Monoxide in Calorimeter Tests", J. Phys. Chem. 1996, 100, pp. 5761-5765.

[19] van Leer, B. Towards the Ultimate Conservative Difference Scheme V: a Second-order Sequel to Godunov's Methods, J Comp. Phys., 32, 1979, pp. 101-136.

[20] Colella, P. \& Glaz, H. M., Efficient Solution Algorithms for the Riemann Problem for Real Gases, J Comp. Phys., 59, 1985, pp. 264-289.

[21] Colella, P. \& P. R. Woodward, P. R., The Piecewise Parabolic Method (PPM) for Gasdynamical Simulations, J Comp. Phys., 54, 1984, pp. 174-201.

[22] Bell, J. B., Colella, P. \& Trangenstein, J. A., Higher Order Godunov Methods for General Systems of Hyperbolic Conservation Laws, J Comp. Phys., 92(2), 1989, pp. 362-397.

[23] Colella, P. "Multidimensional Upwind Methods for Hyperbolic Conservation Laws", $J$ Comp. Phys., 87, 1990, pp. 171-200.

[24] Collins, P., Ferguson, R. E., Chien, K-Y., Kuhl, A. L., Krispin, J. \& Glaz, H. M., Simulation of Shock-Induced Dusty Gas Flows Using Various Models, AIAA 94-2309, 1994.

[25] Berger, M. J. \& Colella, P. Local adaptive mesh refinement for shock hydrodynamics, $J$ Comp. Phys., 82 (1), 1989, pp. 64-84.

[26] Bell, J., Berger, J. M., Saltzman, J., \& Welcome, M., A Three-dimensional adaptive mesh refinement for hyperbolic conservation laws, SIAM J. Sci. Statist. Comput., 15(1), 1994, pp. 127-138.

[27] Pember, R. B., Bell, J. B., Colella, P., Crutchfield, W. Y., \& Welcome, M. L., An Adaptive cartesian grid method for unsteady compressible flow in complex geometries, J Comp. Phys. 120 (2), 1995, pp. 278-304.

[28] Crutchfield, W. Y., \& Welcome, M. L., Object-oriented Implementation of adaptive mesh refinement algorithms, Scientific Programming, 2, 1993, pp. 145-156.

[29] Rendleman, C. A., Beckner, V. E., Lijewski, M., Crutchfield, W. Y., \& Bell, J. B., Parallelization of structured, hierarchical adaptive mesh refinement algorithms, Computing and Visualization in Science, Vol. 3, 2000.

[30] Boris, J. P., On large eddy simulations using sub-grid turbulence models, Wither Turbulence? Turbulence at the Crossroads, ed. J. L. Lumley, Lecture Notes in Physics, 257, Springer-Verlag, Berlin, pp 344-353, 1989.

[31] J. P. Boris, F. F. Grinstein, E. S. Oran, and R. L. Kolbe, New insights into large eddy simulation, Fluid Dynamics Research 10 (1992) pp. 199-229.

[32] Grinstein, F. F., Margolin, L. G., and Rider, W. J., Editors, Implicit Large-Eddy Simulation: Computing Turbulent Fluid Dynamics, Cambridge University Press, 546 pp., 2007.

[33] Aspden, A. J., Nikiforakis, N, Dalziel, S. B. \& Bell, J. B., Analysis of implicit LES methods, Comm. Applied Mathematics and Computational Science, 3, pp.103-126, 2008.

[34] Brode, H. L., A Calculation of the Blast Wave from A Spherical Charge of TNT, Rand Report RM-1965, Rand Corp., Santa Monica, 1957, 61 pp.

[35] Brode, H. L. Blast wave from a spherical charge, Phys. Fluids, 2(2), pp. 217-229 (1959).

[36] Kuhl, A. L. Spherical mixing layers in explosions, Dynamics of Exothermicity, J. R. Bowen Ed., Gordon and Breach, Longhorn, Pennsylvania, pp. 290-320 (+2 color plates), 1996.

[37] G. K. Batchelor, An Introduction to Fluid Dynamics, Cambridge University Press, 1967, $615 \mathrm{pp}$ (vid. esp. \$2.4 Expression for the velocity distribution with specified rate of expansion and vorticity, pp. 85-87).

[38] D. H. Porter, A. Pouquet, and P. R. Woodward, Three-dimensional supersonic homogeneous turbulence: A numerical study, Phys. Rev. Letters 68 (1992) pp. 3156. 


\section{Problem 4. Spherical Mixing Layers}

[39] D. H. Porter, A. Pouquet, and P. R. Woodward, Kolmogorov-like spectra in decaying threedimensional supersonic flows, Phys. Fluids 6 (6) 1994 pp. 2133-2142.

[40] A. N. Kolmogorov, Local structure of turbulence in an incompressible fluid at very high Reynolds numbers, Dokl. Akad. Nauk SSSR, 30 (4) pp. 299-303, 1941.

[41] V. E. Zakharov, V. S. L'vov, G. Falkovich, Komogorov Spectra of Turbulence I: wave turbulence, Springer-Verlag, Berlin, 1992, 264 pp.

[42] A. L. Kuhl, H. Reichenbach, J. B. Bell, V. E. Beckner, Reactive blast waves from composite charges, $14^{\text {th }}$ Int. Detonation Symposium, Office of Naval Research ONR-351-10185 pp. 806-815, 2011. 


\section{Problem 4. Spherical Mixing Layers}

(a) Reactants

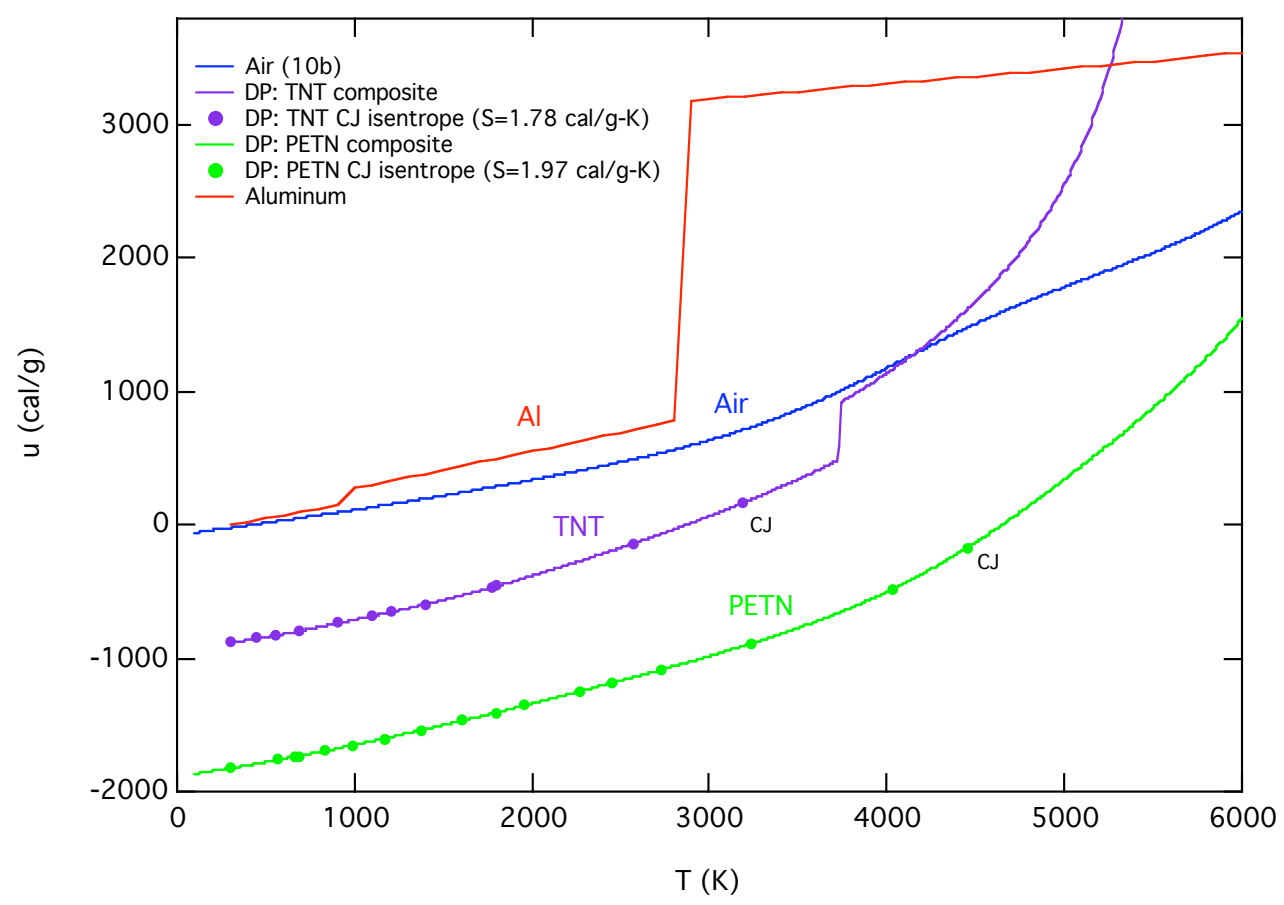

(b) Products

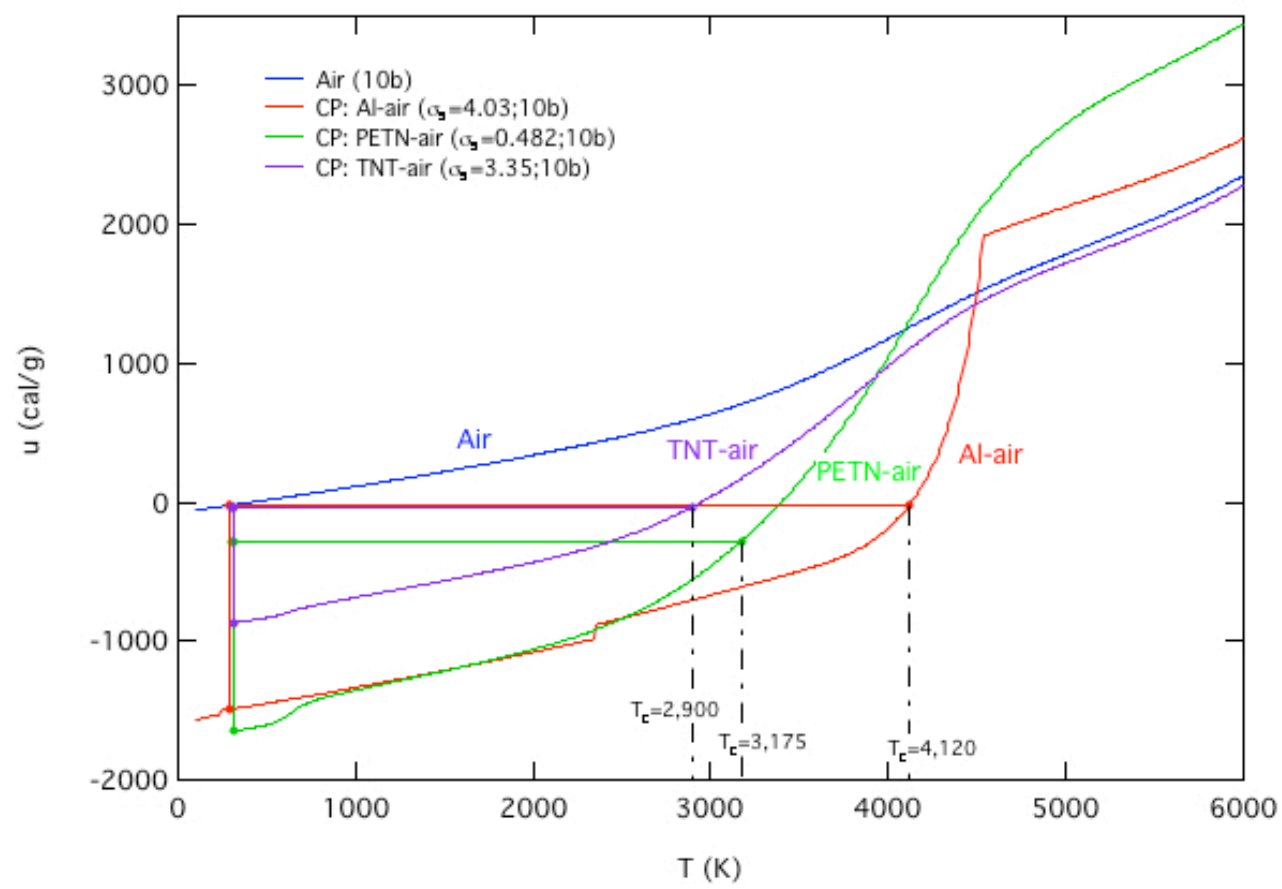

Figure 1. Loci of thermodynamic states in the Le Chatelier plane of specific internal energy-temperature ( $u-T$ plane ): (a) reactants (air, PETN, TNT, Al) at frozen compositon, (b) stoichiometric products (PETN-air, TNT-air, Al-air) in thermodynamic equibrium. 


\section{Problem 4. Spherical Mixing Layers}

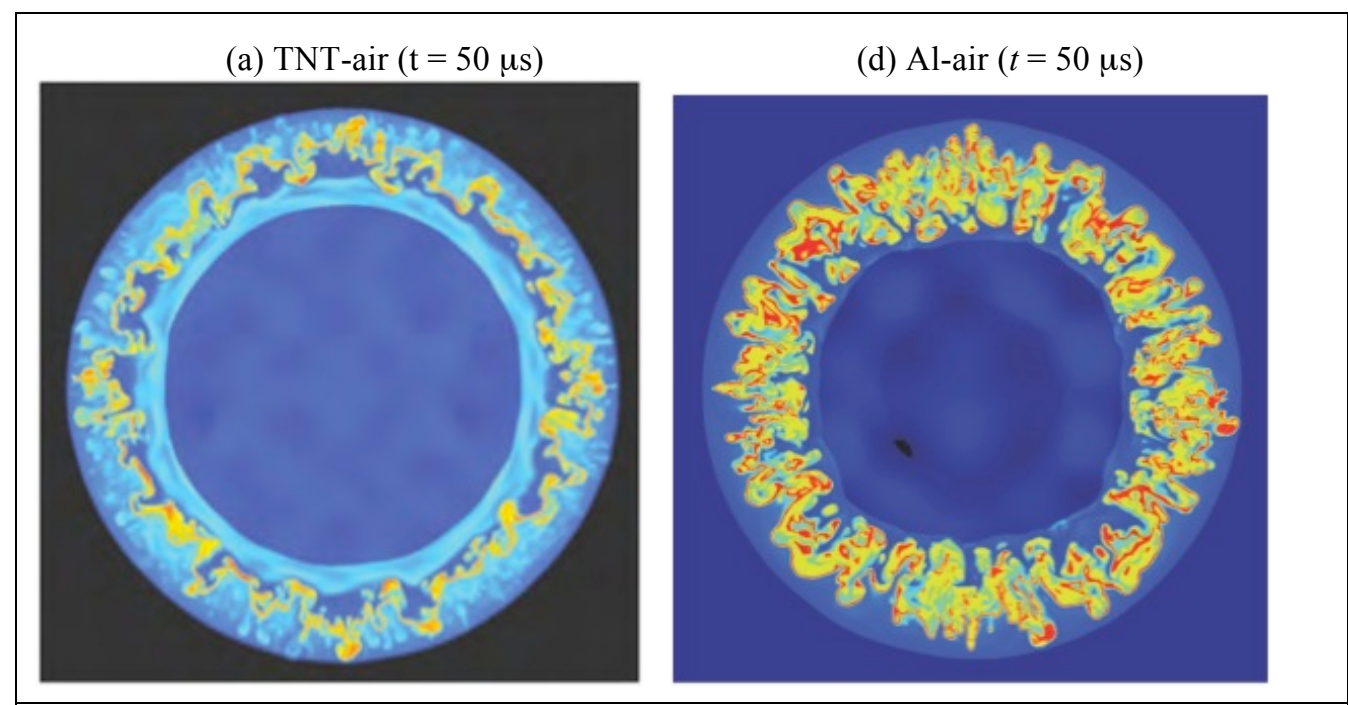

(b) TNT-air $(t=110 \mu \mathrm{s})$

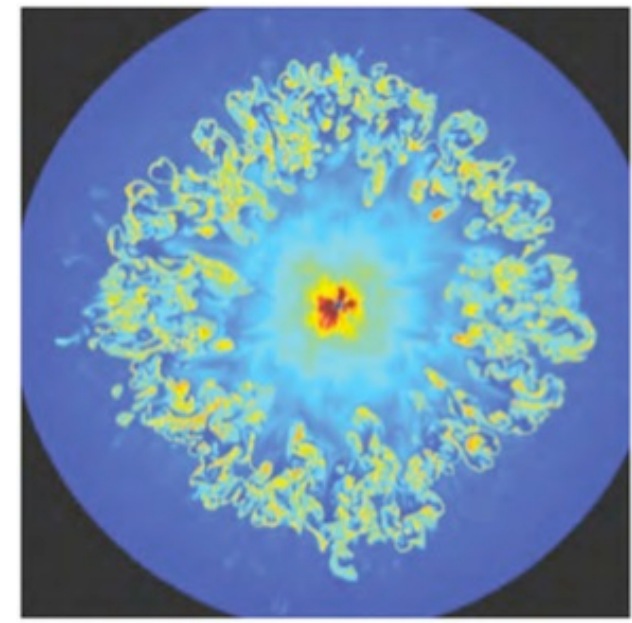

(e) Al-air $(t=110 \mu \mathrm{s})$

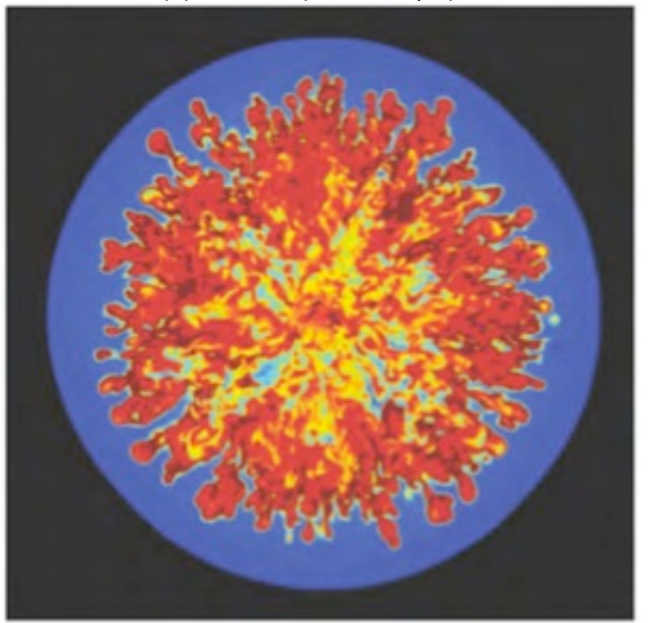

(c) TNT-air $(t=410 \mu \mathrm{s})$

(f) Al-air $(t=410 \mu \mathrm{s})$
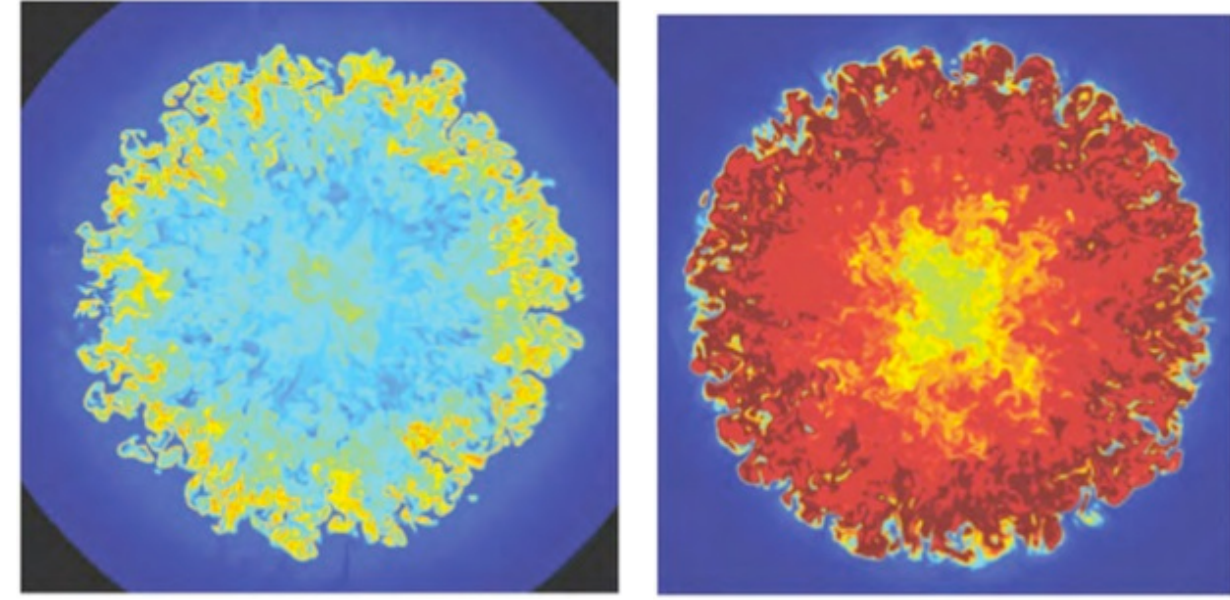

Figure 2. Cross-sectional view of the temperature field of spherical combustion clouds: frame $a$ - $c$ illustrate TNT-air combustion while frames $d$ - $f$ depict Al-air combustion (colors denote temperature — red: 3,000< $T<4,000 \mathrm{~K}$; yellow: $T=2,000 \mathrm{~K}$; turquoise: $\mathrm{T}=1,000 \mathrm{~K}$ ). 


\section{Problem 4. Spherical Mixing Layers}

(a) flow field

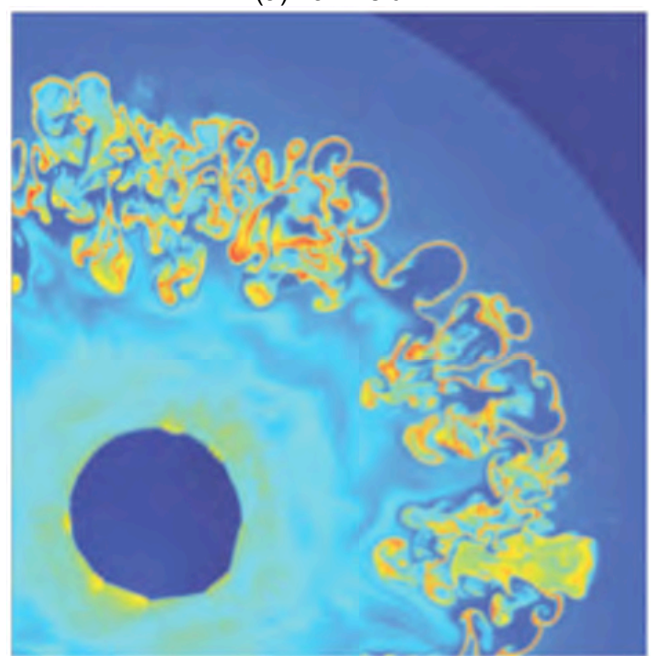

(b) blow-up near front

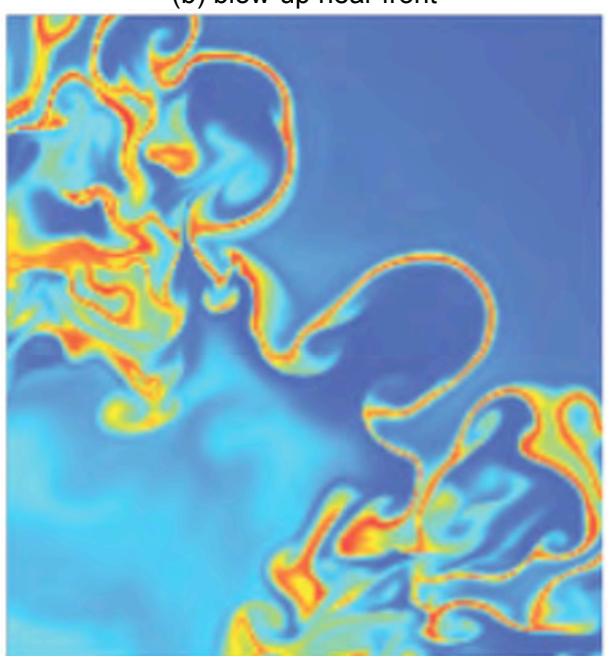

Figure 3. Exothermic flame sheet in the TNT-air combustion field $(t=100 \mu \mathrm{s})$.

(a) TNT explosion in air

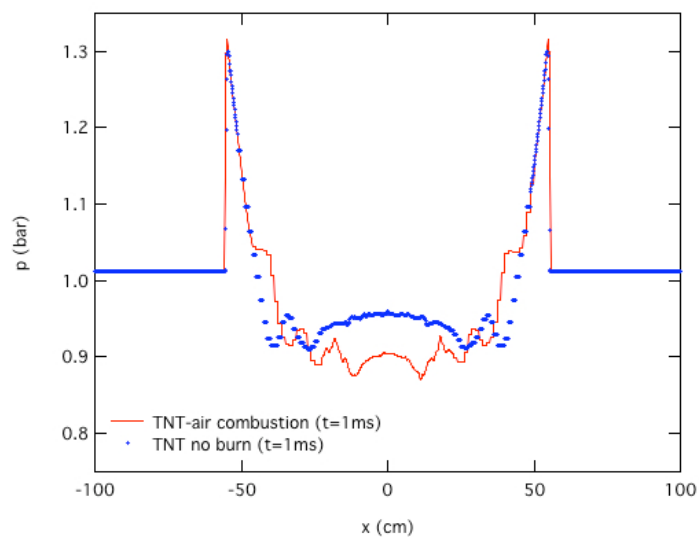

(b) AI-SDF explosion in air

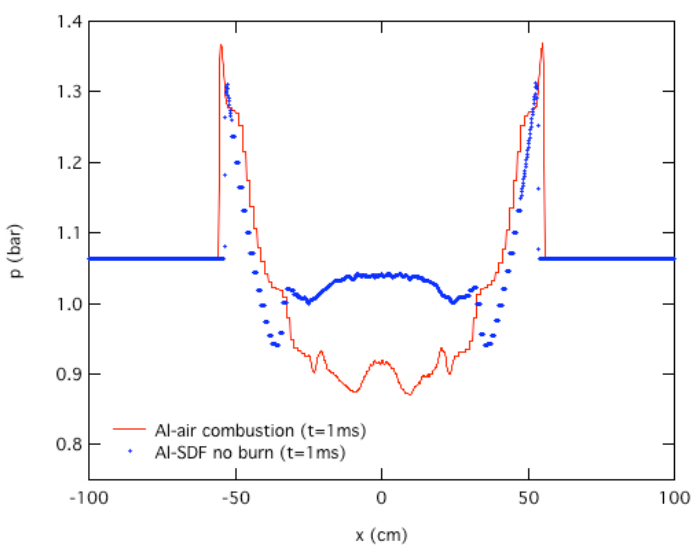

Figure 4. Blast wave pressure distribution $p(x, t=1 \mathrm{~ms})$ along the $x$-axis at for explosions in air: (a) TNT explosion in air, (b) Al-SDF explosion in air. Notation: solid curve denotes combustion with air while dotted curve represents the no combustion case.

(a) TNT-air combustion

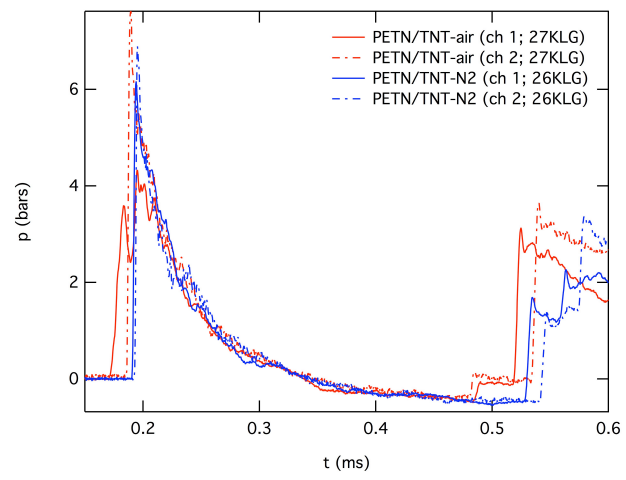

(b) Al-air combustion

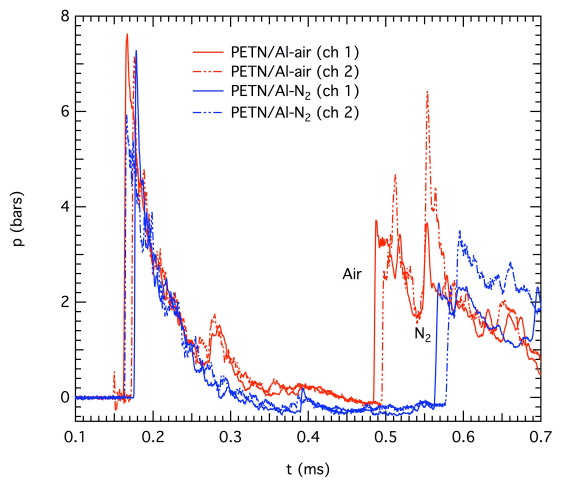

Figure 5. Blast wave pressure histories measured on the lid of calorimeter $\mathrm{C}$ for TNT and Al SDF charges; red curves represent combustion in air while blue curves represent the explosions in $\mathrm{N}_{2}$ [42]. 


\section{Problem 4. Spherical Mixing Layers}

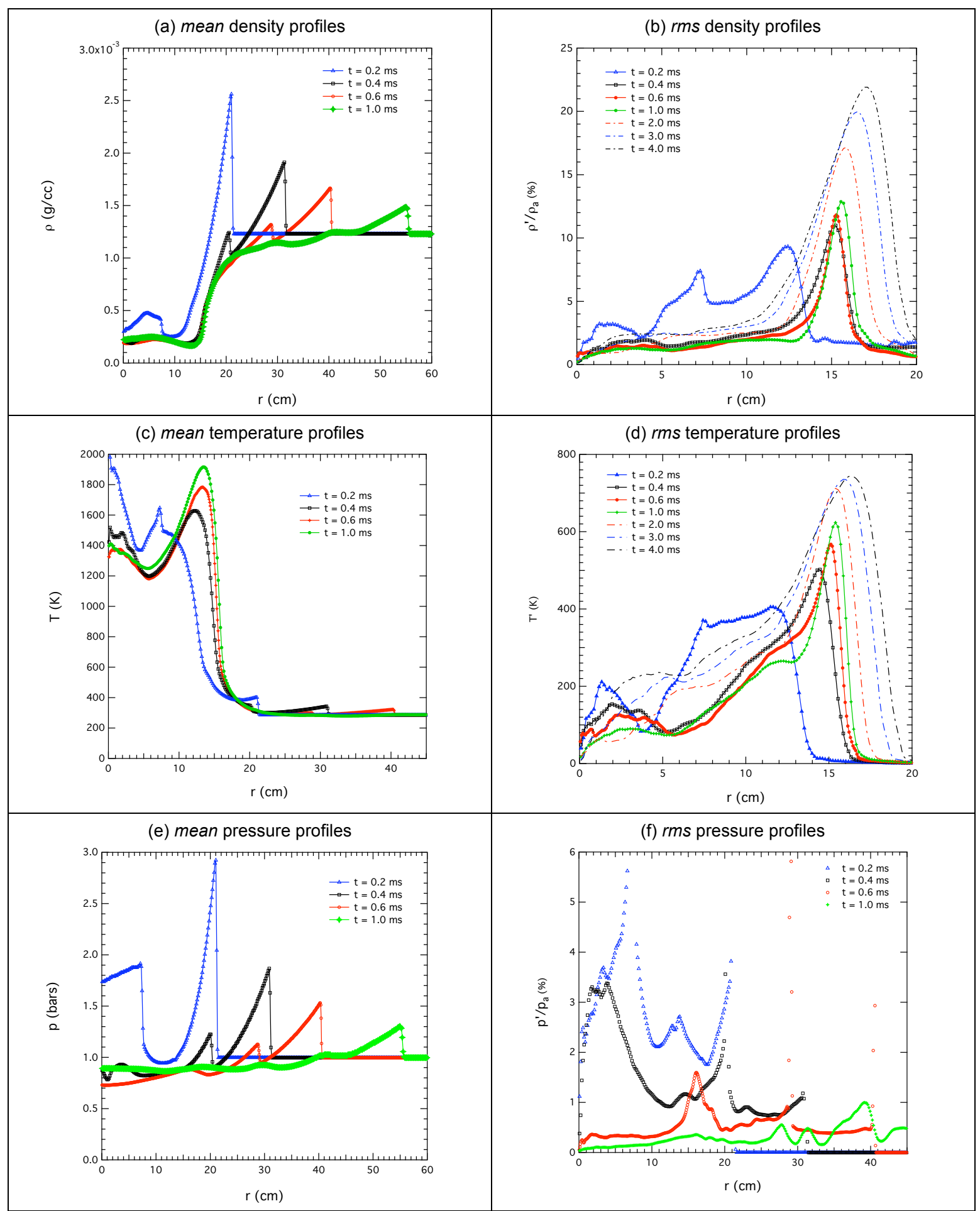

Figure 6. Evolution of the mean and rms thermodynamic profiles in the TNT combustion cloud. 


\section{Problem 4. Spherical Mixing Layers}

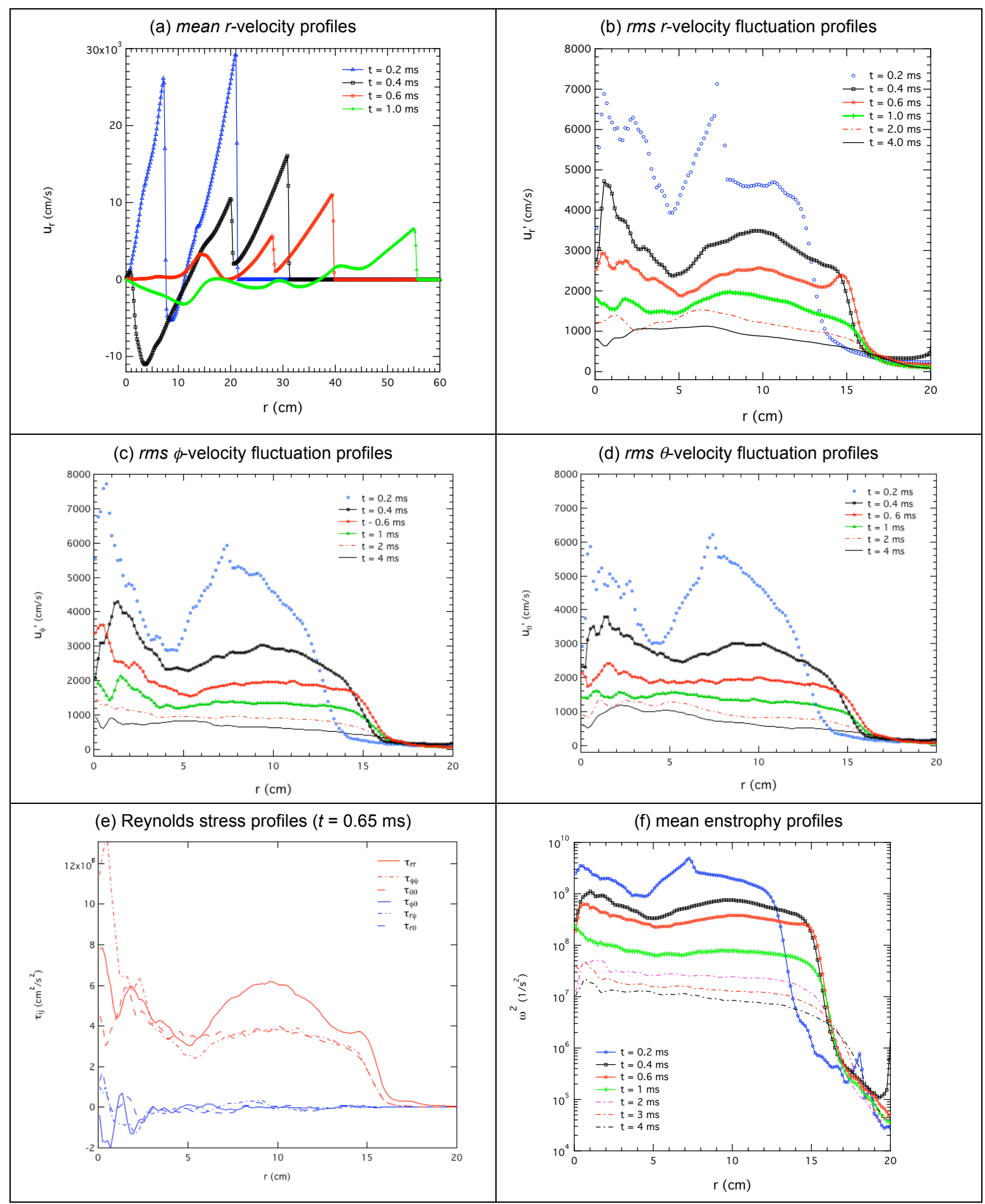

Figure 7. Evolution of the mean and rms kinematics profiles in the TNT combustion cloud. 
Problem 4. Spherical Mixing Layers

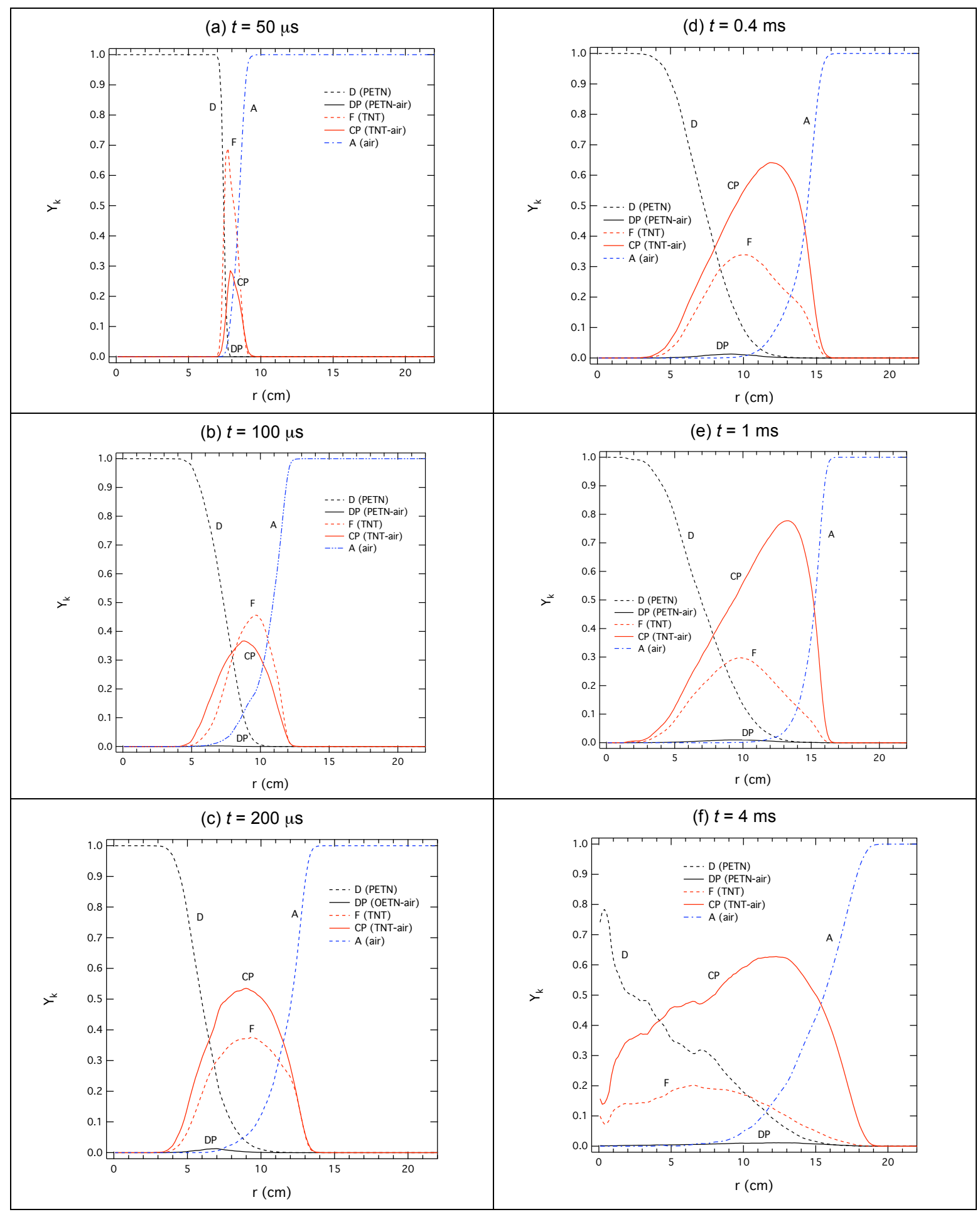

Figure 8. Evolution of the mean mass-fraction profiles $Y_{k}(r, t)$ in the combustion zone of the TNT cloud (Notation: D $=$ PETN driver, $\mathrm{DP}=\mathrm{PETN}$-air combustion products, $\mathrm{F}=\mathrm{TNT}$ fuel, $\mathrm{CP}=\mathrm{TNT}$-air combustion products, $\mathrm{A}=$ air $)$. 


\section{Problem 4. Spherical Mixing Layers}

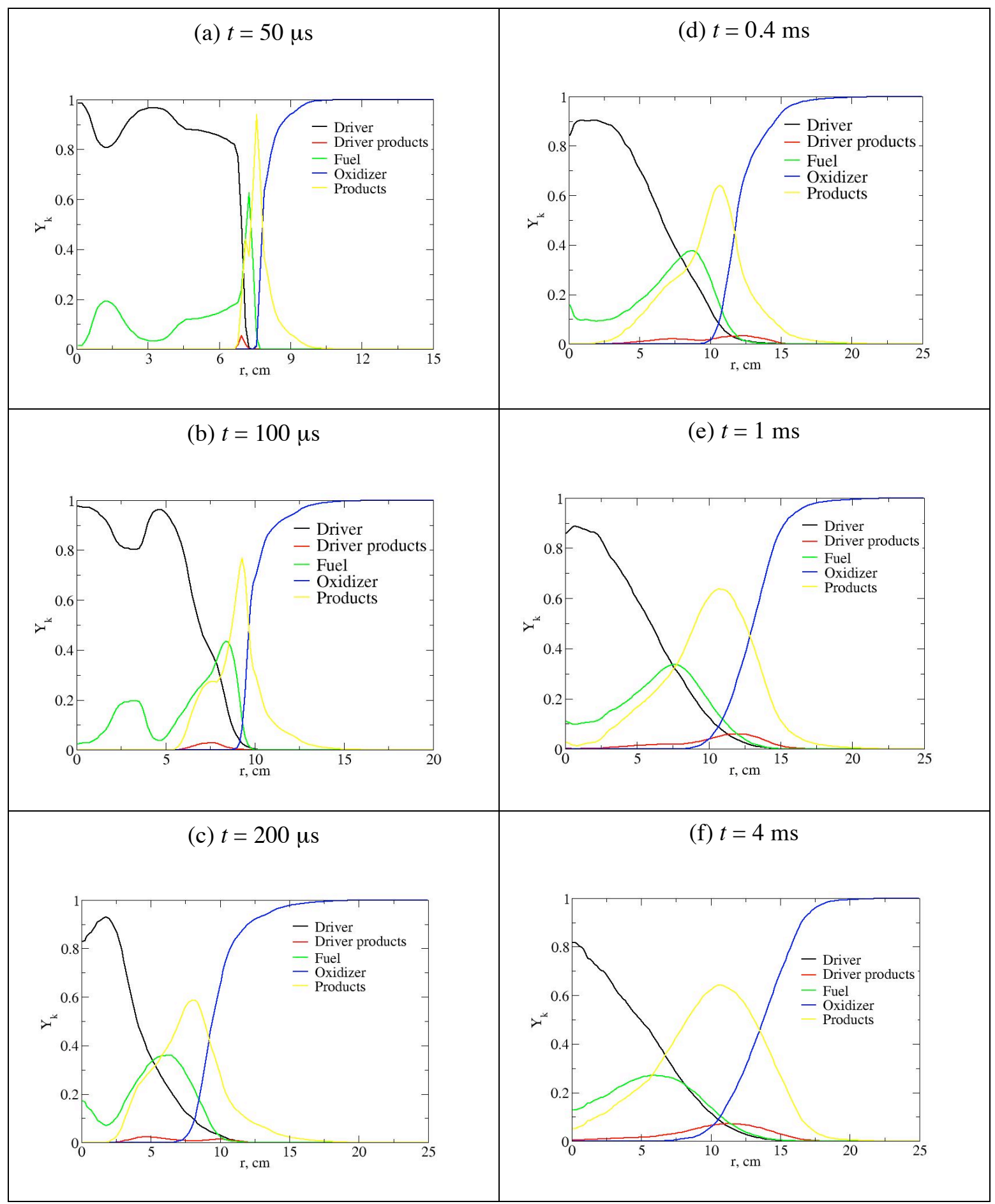

Figure 9. Evolution of the mean mass-fraction profiles $Y_{k}(r, t)$ in the combustion zone of the Al-air cloud (Notation: $\mathrm{D}=$ PETN driver, $\mathrm{DP}=\mathrm{PETN}$-air combustion products, $\mathrm{F}=\mathrm{Al}$ fuel, $\mathrm{CP}=\mathrm{Al}$-air combustion products, $\mathrm{A}=$ air $)$. 


\section{Problem 4. Spherical Mixing Layers}

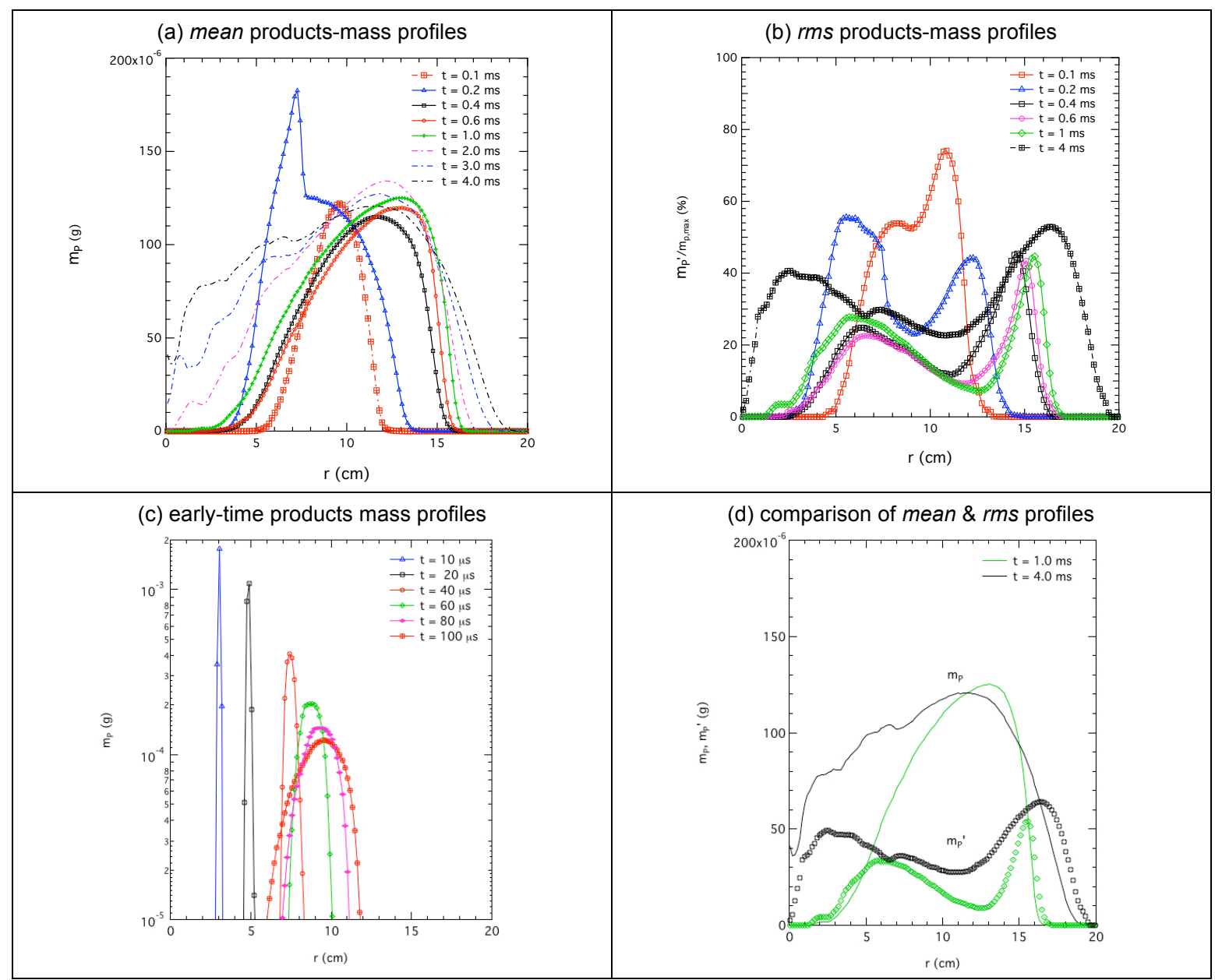

Figure 10. Evolution of the mean and rms profiles of the combustion Products mass in the TNT combustion cloud.

(a) TNT-air combustion

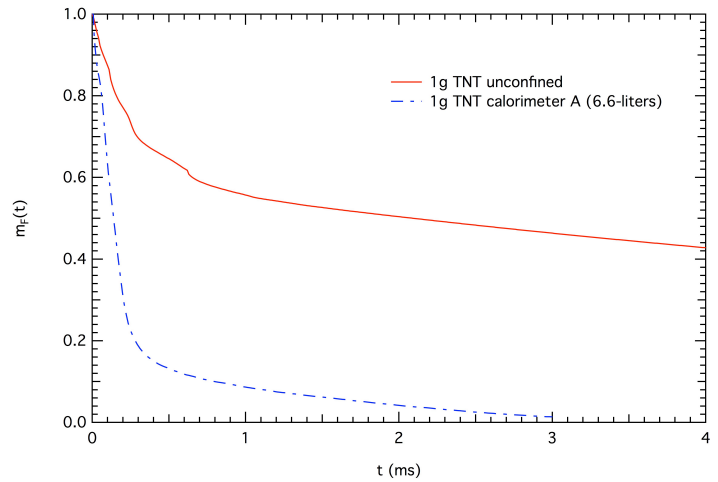

(b) Al-air combustion

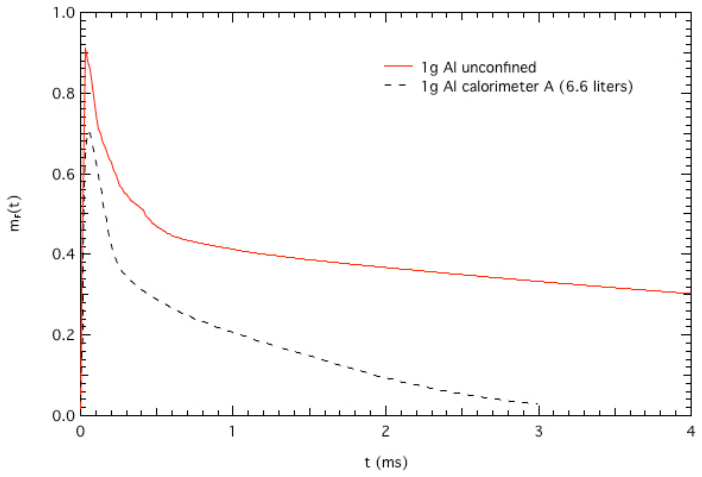

Figure 11. Fuel consumption over time: (a) TNT-air combustion; (b) Al-air combustion. 


\section{Problem 4. Spherical Mixing Layers}

(a) early-time growth

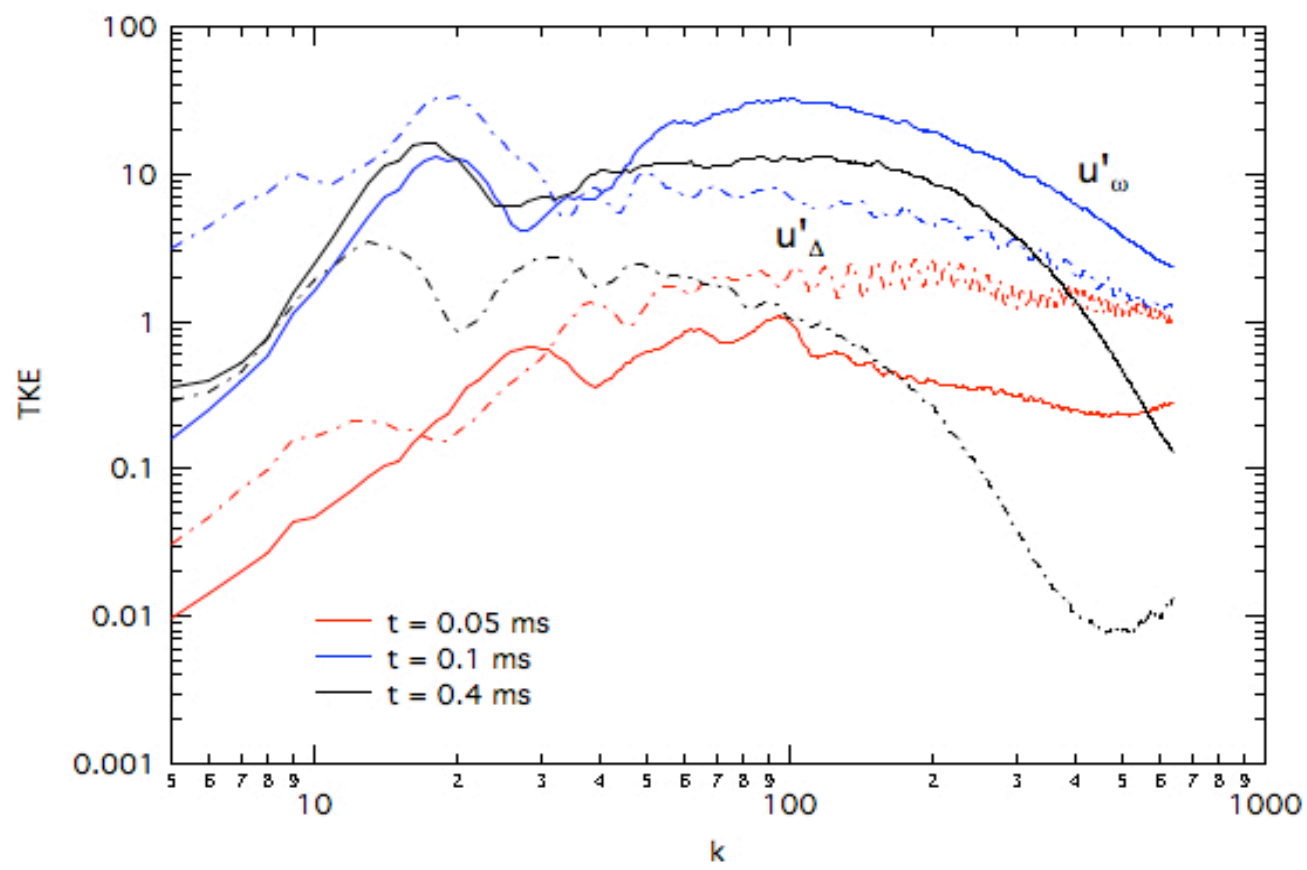

(b) decay

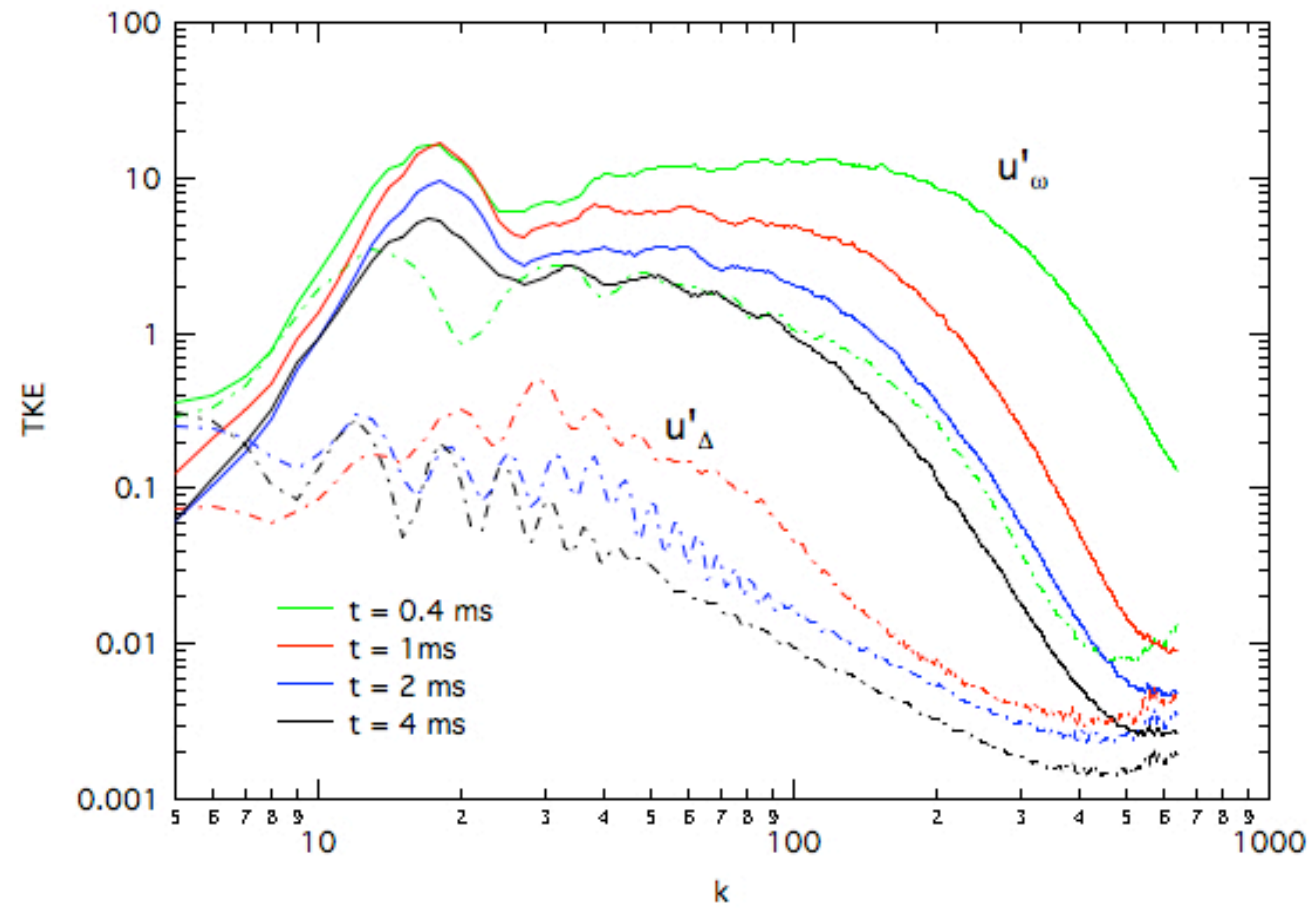

Figure 12. Evolution of the Turbulent Kinetic Energy (TKE) spectrum in the TNT-air combustion cloud: (a) earlytime growth, (b) decay. Solid lines denote the rotational velocity component, dashed lines represent the dilatational velocity component. 


\section{Problem 4. Spherical Mixing Layers}

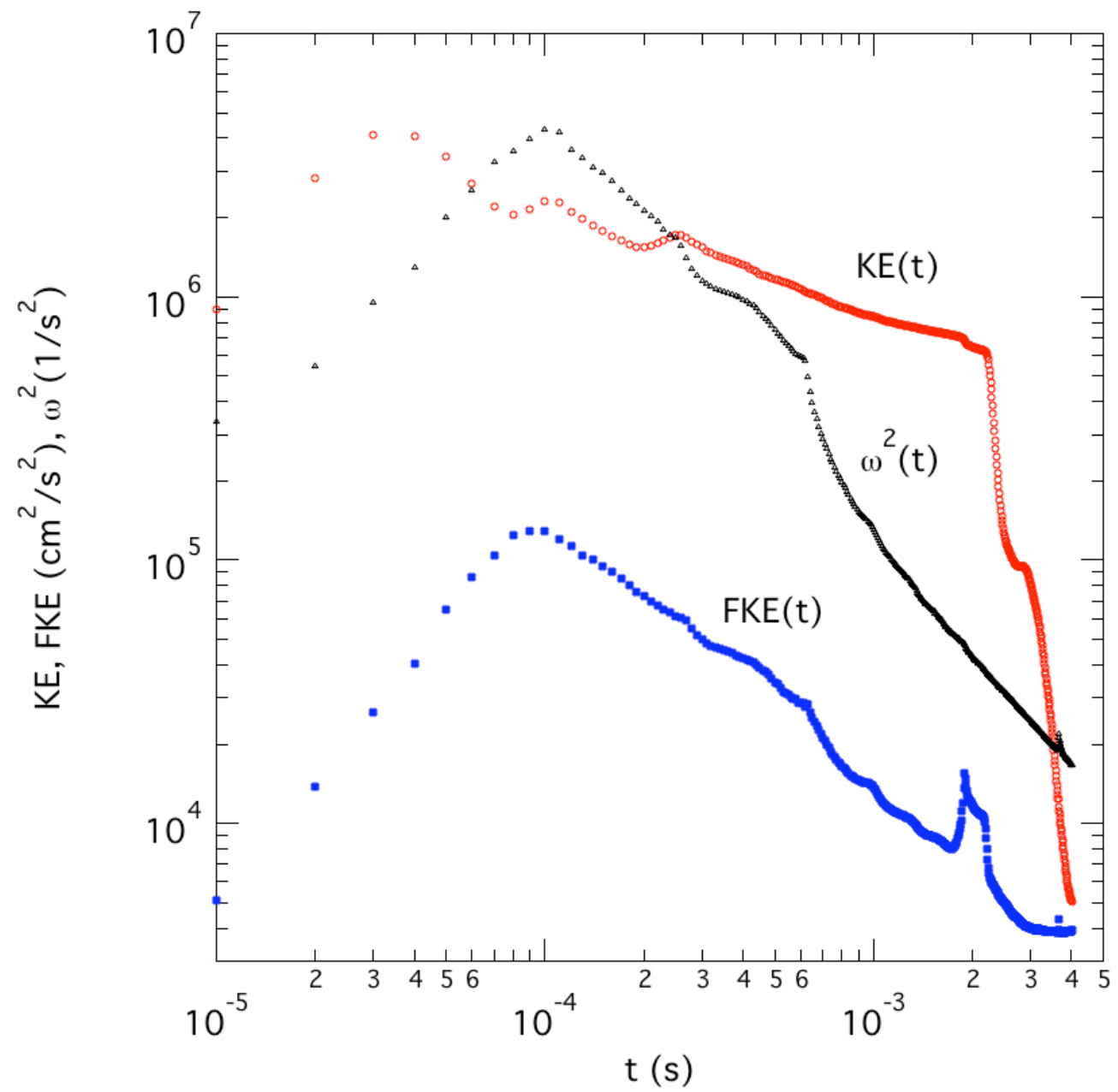

Figure 13. Evolution of the global kinetic energy, fluctuating kinetic energy and enstrophy in the TNT explosion. 


\title{
Problem 5: Kelvin-Helmholtz Mixing Layers ${ }^{2122}$
}

\begin{abstract}
SUMMARY
The dynamics of unconfined. spatially developing shear layers is studied through the numerical solutions of the time-dependent Euler equations using a second-order Godunov scheme. The effects of density and velocity variations between the two streams of the shear layer are studied and color graphics are used to show more clearly the entrainment process of the surrounding streams. The calculations demonstrate that the evolution of the mean flow is dominated by two-dimensional, inviscid effects. The r.m.s. fluctuating velocity and density profiles are in good agreement with the measurements of Oster and Wygnanski and of Konrad, except for the peak value of the $v^{\prime}$ profile.
\end{abstract}

\section{INTRODUCTION}

This paper presents the results of numerical simulations of unconfined, spatially developing, two-dimensional unsteady shear layers in a constant ambient-pressure environment. It has been known for more than a hundred years that the shear layer generated by a splitter plate can be inviscidly unstable. The late-time consequence of such flow instabilities is that the perturbed shear layers roll up into large-scale vortical structures (Michel, 1932; Brown and Roshko, 1974); the latter work also demonstrate that the largest-scale structures are essentially independent of the flow Reynolds number (although micro-scale mixing within these structures is Reynolds-number

${ }^{21}$ A. L. Kuhl, K.Y. Chien, R. E. Ferguson, H. M. Glaz, P. Colella, Inviscid dynamics of unstable shear layers, RDA-TR-161604-006, August 1988, 94 pp.

${ }^{22}$ K-Y. Chien, R. E. Ferguson, A. L. Kuhl, H. M. Glaz, P. Colella, Inviscid dynamics of two-dimensional shear layers, Int. J. Computational Fluid Dynamics, 1995, 5 (1-2), p. 59-80. 


\section{Problem 5. Kelvin-Helmholtz Mixing Layers}

dependent). It also is known that the growth of the shear layer is governed by the pairing mechanism of these vortical structures (Winant and Browand, 1974), a complex non-linear dynamical process (Corcos and Sherman, 1984). Perturbations may occur naturally or may be intentionally applied. Studies with applied upstream perturbations indicate that vortex dynamics is basically inviscid (Ho and Huang, 1982; Oster and Wygnanski, 1982).

Many shear layer computations involving discretizations of the Navier-Stokes equations are available. These include results using finite-difference methods (Corcos and Sherman, 1984; McInville et al., 1985; Davis and Moore, 1985), spectral methods (Riley and Metcalfe, 1980), and vortex methods (Ashurst, 1979; Leonard, 1980; Ghoniem et al., 1982). All of these calculations succeed in resolving small-scale flowfield structures and are of high quality. However, modeling of the viscous terms imposes computational inefficiencies due to the disparity between viscous and inviscid length scales. One may either resolve the viscous length scales on a mesh and, hence, use severely restricted time steps, or employ implicit time differencing and accept the resulting numerical diffusion. The random vortex method (Chorin 1973, 1986) avoids this problem at the expense of introducing a statistical error.

Due to advances in the development of high-order upwind methods for solving conservation laws, it is now possible to compute high resolution, essentially non-diffusive flowfields for the equations of inviscid gas dynamics. This approach is taken here to simulate shear layer experiments with the objective of demonstrating that viscous forces need not be taken into account in modeling the dynamics of shear layer flowfields. Such methods neglect molecular diffusion effects (i.e., diffusion of vorticity and passive scalars across streamlines), so that the methodology may be viewed as an asymptotically large Reynolds number approach. An advantage of using the gas dynamic equations is that baroclinic generation of vorticity is automatically included. The main disadvantage is that small Mach number calculations can be quite expensive; this will be explained in more detail in Section 2.

Previous calculations along these lines have been reported by Grinstein et al. (1986), using a flux-corrected transport code to solve the Euler equations, and by Inoue (1985), using a vortex method to solve potential equations. Obviously, steady upstream conditions will not produce any vortex formation using an Euler code. A fluctuating inflow-pressure condition was implemented by Grinstein et al. (1986) by imposing a zero-slope condition on the pressure at the inflow boundary. Therefore the pressure perturbations are not prescribed; in fact, the quantitative values of these perturbations are part of the solution. We chose to follow our previous work (Chien et al., 1987) by imposing a given velocity perturbation at the inflow (upstream) boundary, with the frequency content and relative amplitudes of the perturbations obtained from the inviscid linear spatial instability calculations of Monkewitz and Huerre (1982). (More details are given in Sec. 2.) In addition, quantitative r.m.s. fluctuating velocity results, which require long computations for converged solutions, are not available in Grinstein et al. (1986). In fact, despite the large amount of computational efforts, detailed quantitative mean and fluctuating flow calculations are quite meager; all of those reported in the literature have been based on constant-density vortex methods (Ashurst, 1979; Inoue, 1985; Inoue and Leonard, 1987; Ghoniem and Ng, 1987). Recently, a variable-density vortex method has been presented by Soteriou et al.(1991); however, no mean and fluctuating density profiles were reported by them. In fact, our 


\section{Problem 5. Kelvin-Helmholtz Mixing Layers}

paper is the only one, so far, that presents both such profiles and compares them with the measurements of Brown and Roshko (1974) and Konrad (1977). Therefore, the primary purpose of this paper is to present detailed quantitative results based on a finite-difference solution of the Euler equations and to compare these solutions with available experiments and other pertinent computations. The effects of both the density ratio and the velocity ratio on the solutions are addressed in detail in the paper.

The numerical results presented here were obtained with an explicit second-order Godunov scheme (Colella and Glaz, 1985). The non-diffusive, high resolution properties of this scheme have been carefully demonstrated by a mesh refinement study combined with a comprehensive comparison with other schemes (Woodward and Colella, 1984). Its accuracy has been shown in a variety of applications (Colella et al., 1985; Glaz et al., 1985); in many situations, embedded shear layers become unstable, roll up, and interact with the ambient flow as well as self-interact (Glowacki et al., 1986; Kuhl et al., 1987). Thus, the present calculations also serve to validate the accuracy of the code in shear layer regions, by direct qualitative and quantitative comparison with experimental data.

By design, our work is limited to unsteady calculations of two-dimensional shear layers which are statistically steady (in the sense of time-averaging); as suggested by Roshko (1981), a two-dimensional solution is valuable both as a gauge against which to compare the real flows, and as a step in understanding them. Extension of the present method to three-dimensional calculations using operator splitting is conceptually feasible, but the computational resources required for such an effort is prohibitive at the present time. Some of the results herein have been presented elsewhere in preliminary versions of this work (Chien et al., 1988, 1989; Kuhl et al., 1990). In these earlier reports, monitoring stations situated at three constant streamwise locations were used to store the flowfield time histories for quantitative data analysis. Since the upstream condition is known to have a strong effect on the development of a free shear layer (Bradshaw, 1966), more monitoring stations are needed to study the rate at which the self-similarity of the various mean and fluctuating profiles is attained. Nineteen monitoring stations located at constant streamwise intervals throughout the region of interest have been used in three new calculations to be presented in this study. Two of these calculations correspond to the cases studied experimentally in detail by Brown and Roshko (1974) and by Oster and Wygnanski (1982), respectively. The density ratio and the velocity ratio for these two cases are both different. Since the development of a free shear layer is

known to depend on both of these ratios, a third calculation has also been carried out to assess the effect of each parameter individually.

The formulation of our numerical simulations is described in Section 2. Results and comparison with experimental data are presented in Section 3, where detailed quantitative comparisons of mean and fluctuating flowfield profiles are offered (the numerical data are obtained by statistically averaging the numerical solution over time intervals which are long relative to the time scales of vortex formation and pairing). Our conclusions are summarized in Section 4.

\section{METHODOLOGY}

Our computational models are based on the equations of inviscid gas dynamics, thereby neglecting the molecular viscosity and heat conduction; the gravitational 


\section{Problem 5. Kelvin-Helmholtz Mixing Layers}

forces are also neglected. The equations may be written in conservation form for a Cartesian geometry as

$$
\mathbf{U}_{t}+\mathbf{F}(\mathbf{U})_{x}+\mathbf{G}(\mathbf{U})_{y}=0
$$

where

$$
\mathbf{U}=\left(\begin{array}{c}
\rho \\
\rho u \\
\rho v \\
\rho E
\end{array}\right), \quad \mathbf{F}=\left(\begin{array}{c}
\rho u \\
\rho u^{2}+p \\
\rho u v \\
\rho u E+u p
\end{array}\right), \quad \mathbf{G}=\left(\begin{array}{c}
\rho v \\
\rho u v \\
\rho v^{2}+p \\
\rho v E+v p
\end{array}\right)
$$

and $\rho$ is the density, $\mathbf{u}=(u, v)$ is the velocity field in the Cartesian coordinates $\mathbf{x}=(x, y)$, $p$ is the pressure, $E=e+\frac{1}{2}\left(u^{2}+v^{2}\right)$ is the total specific energy, and $e$ is the specific internal energy. We always assume a perfect gas with the equation-of-state

$$
p=(\gamma-1) \rho e
$$

where $\gamma=1.4$. The streamwise direction is always the positive $x$-axis.

The Eulerian second-order Godunov scheme (Colella and Glaz, 1985) is used to solve these equations for given initial data and boundary conditions. This scheme is in conservation form and explicit.

Dimensional operator splitting is used for two-dimensional calculations. A rectangular $N \times M$ mesh is specified by defining the zone interfaces $x_{i}, i=0, \ldots, N$ and $y_{j}, j=0, \ldots, M$ with zone sizes $\Delta x_{i}$ and $\Delta y_{j}$ where $\Delta x_{i}=x_{i}-x_{i-1}$ and $\Delta y_{j}=y_{j}-y_{j-1}$. The flowfield quantities, averaged over a computational zone, are denoted by $\rho_{i j}$, etc. The time step is limited by the CFL stability criterion

$$
\Delta t=\sigma \min _{i, j}\left(\frac{\Delta x_{i}}{\left(c_{i j}+\left|u_{i j}\right|\right)}, \frac{\Delta y_{j}}{\left(c_{i j}+\left|v_{i j}\right|\right)}\right)
$$

where $c=(\gamma p / \rho)^{1 / 2}$ is the sound speed and $\sigma<1$ is the Courant number. The value $\sigma=0.95$ has been used in all calculations.

Such explicit methods are most appropriate for compressible flowfields. Small Mach number flowfields may also be obtained, but at the cost of either very poor computational efficiency or violation of the Mach number similitude. For example, if the Mach number $M \sim 0.01$ (which is typical of some of the experimental data used here), then it would take approximately one hundred time steps to convect a fluid parcel through one computational zone. To avoid this problem, the sound speed of the gas has been modified for the computations so that typical flow Mach numbers would be of the order $M \sim 0.2$. Of course, the density and velocity ratios across the shear layer are preserved, but the Mach number similitude is violated. Since the compressibility effects are negligible at these Mach numbers and we do not require temperature data for our comparisons, this Mach number compromise is acceptable for the present study.

The dynamics of the interface separating the two fluid streams is of great importance in shear layer studies. Consequently, an additional calculation was performed of 


\section{Problem 5. Kelvin-Helmholtz Mixing Layers}

a discretization of the material interface by marker particles, $\mathbf{x}_{k}(t), k=1, \ldots, K(t)$ where $\mathbf{x}_{k}(t)$ denotes the location of the $k$ th marker at time $t$ and $K(t)$ is the total number of markers present in the flowfield at time $t$. The time evolution of the markers was governed by passive advection, i.e., $d \mathbf{x}_{k} / d t=\mathbf{u}\left(\mathbf{x}_{k}, t\right)$; this ordinary differential equation, with the right-hand side given by the underlying computed velocity field, was solved by straightforward finite differences and a linear interpolation for $\mathbf{u}$. Initially, the interface is represented by several thousand markers and new markers are inserted at the inflow during every time step. Since the interface is unstable, it is expected that $\left|\mathbf{x}_{k}-\mathbf{x}_{k-1}\right|$ can become large; whenever this value exceeds twice the initial marker spacing, a new marker is inserted at their midpoint. Marker points which exit the fine-grid region (see below) are deleted. Finally, the condition $K(t) \leqslant 75,000$ is imposed due to computer time limitations; if the condition is violated, no further markers are inserted over the last forty percent of the mesh in the streamwise direction.

The accuracy of this approach is a matter of some debate. Due to the underlying physical instability, one is solving what amounts to a chaotic dynamical system in current terminology. In particular, the exponential separation of orbits is expected, which implies that the numerical orbit $\mathbf{x}_{k}(t)$ is not close to the actual physical orbit of the initial marker after a time which is probably small relative to the total computation time. However, this does not imply that the aggregate of the numerical orbits is not a good representation of the aggregate of the physical orbits. Indeed, our results here (comparisons with contour plots and shadowgraphs from the experiments) suggest the contrary.

The calculations were performed on a two-dimensional Cartesian grid, see Figure 1. A fine, uniformly-zoned region with $\Delta x=\Delta y$ covering the domain $D_{F}=\{(x, y): 0 \leqslant$ $\left.x \leqslant X_{F},-Y_{F} \leqslant y \leqslant Y_{F}\right\}$ straddles the centerline of the shear layer. An expanding grid (above, below, and to the right of $D_{F}$ ) was used to place the mesh boundaries far from the region of interest and thereby minimize any influence of the numerical boundary condition on the dynamics of the free shear layer. For this study, we have used $\Omega_{F}=500 \times 80$ and $\Omega_{r}=520 \times 114$, where $\Omega_{F}$ and $\Omega_{T}$ refer to the grids in the fine and total mesh regions, respectively.

The initial data was determined using a $\operatorname{Tanh}(y)$ profile to model the shear layer. Hence

$$
\begin{aligned}
& u(x, y, t=0)=f(y) \\
& v(x, y, t=0)=0
\end{aligned}
$$

where

$$
f(y)=U_{m}[1+\lambda \operatorname{Tanh}(2 y / \delta)]
$$

and

$$
U_{m}=\frac{\left(U_{1}+U_{2}\right)}{2}, \quad \lambda=\frac{\left(U_{1}-U_{2}\right)}{\left(U_{1}+U_{2}\right)}
$$

Here $U_{1}$ and $U_{2}$ denote the free stream velocity at $y= \pm \infty$, respectively; $U_{m}$ is the mean flow velocity; $\lambda$ is the shear parameter and $\delta$ is the maximum slope thickness of the layer, i.e., $\delta=\left(U_{1}-U_{2}\right) /(\mathrm{d} u / \mathrm{d} y)_{\max }$. If the free stream densities are different, we have

$$
\rho(x, y, t=0)=h(y)
$$




\section{Problem 5. Kelvin-Helmholtz Mixing Layers}

where

$$
\begin{aligned}
& h(y)=\rho_{m}\left[1+\lambda_{\rho} \operatorname{Tanh}(2 y / \delta)\right] \\
& \rho_{m}=\frac{\left(\rho_{1}+\rho_{2}\right)}{2}, \quad \lambda_{p}=\frac{\left(\rho_{1}-\rho_{2}\right)}{\left(\rho_{1}+\rho_{2}\right)},
\end{aligned}
$$

where $\rho_{1}, \rho_{2}$ are the free stream densities at $y= \pm \infty$, respectively; $\rho_{m}$ is the mean shear layer density and $\lambda_{p}$ is the density shear parameter. Note that the same $\delta$ is used as the length scale for the density distribution.

The left inflow boundary data are determined from

$$
\begin{gathered}
u(0, y, t)=f(y) \cdot g(t) \\
v(0, y, t)=0 \\
\rho(0, y, t)=h(y)
\end{gathered}
$$

where

$$
g(t)=1+\sum_{n=1}^{N} \varepsilon_{n} \sin \left(\omega_{n} t\right)
$$

The perturbation frequencies $\omega_{n}$ and amplitudes $\varepsilon_{n}$ have been taken from the linear stability analysis for $\operatorname{Tanh}(y)$ shear layer profiles (Monkewitz and Huerre, 1982):

$$
\begin{aligned}
& \omega_{1}=0.438 ; \quad \omega_{n}=\omega_{1} / n, \quad n=1, \ldots, N, \\
& \varepsilon_{1}=0.01, \quad \varepsilon_{2}=0.75 \varepsilon_{1}, \quad \varepsilon_{3}=0.55 \varepsilon_{1}, \quad \varepsilon_{4}=0.44 \varepsilon_{1}, \\
& \varepsilon_{n}=1.7 \varepsilon_{1} / n \quad \text { for } \quad n \geqslant 5 .
\end{aligned}
$$

Note that the maximum perturbation amplitude is one percent and $\omega_{1}$ is twice the value given by Monkewitz and Huerre (1982) because our reference length scale is twice theirs (see below).

Numerical implementation of the boundary conditions is straightforward (Colella and Glaz, 1985) for free shear layers. Dirichlet data is imposed on the upstream side (using the equations above) as well as the top and bottom sides (using free stream values) and an outflow boundary condition is used on the downstream side.

The length has been normalized by $\delta / 2$ and we choose $\Delta x=\Delta y=1$ in the fine zone region; i.e., $X_{F}=500$ and $Y_{F}=40$. Also, $X_{R}=8800$ and $Y_{T}=1500$ (see Fig. 1 for definitions of $X_{F}, X_{R}, Y_{F}$ and $Y_{T}$ ); it may be noted how rapidly the grid expands from the fine mesh to cover the total computational domain. Despite the excellent agreement with the experiment, it would be of great interest to perform a mesh refinement study and re-analyze the detailed flowfield structures. With the code used here, this is currently beyond the limits of our computer resources.

The physical parameters defining the simulations are summarized in Table 1 . The parameter $r$ is defined by $r=U_{2} / U_{1}$ while $M_{1}, M_{2}$ and $M_{m}$ refer to the Mach numbers 


\section{Problem 5. Kelvin-Helmholtz Mixing Layers}

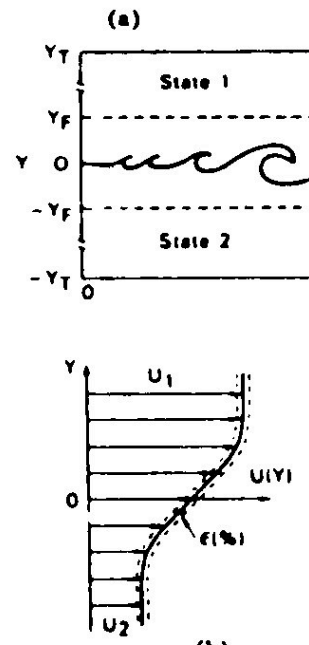

(b)

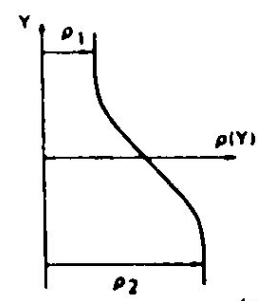

(c)

Figure 1 Schematic of the free shear layer calculations: (a) computational grid; (b) Tanh (y) streamwise velocity profile and (c) density profile.

Table 1 Test case parameters

\begin{tabular}{lllllllllll}
\hline Case & $U_{1}$ & $U_{2}$ & $r$ & $\lambda$ & $\rho_{1}$ & $\rho_{2}$ & $\lambda_{\rho}$ & $M_{1}$ & $M_{2}$ & $M_{m}$ \\
\hline I & 1.45 & 0.55 & 0.38 & 0.45 & 1.0 & 7.0 & -0.75 & 0.29 & 0.29 & 0.40 \\
II & 1.45 & 0.55 & 0.38 & 0.45 & 7.0 & 1.0 & 0.75 & 0.77 & 0.11 & 0.40 \\
III & 1.25 & 0.75 & 0.60 & 0.25 & 1.0 & 1.0 & 0 & 0.25 & 0.15 & 0.20 \\
IV & 1.45 & 0.55 & 0.38 & 0.45 & 1.0 & 1.0 & 0 & 0.29 & 0.11 & 0.20 \\
\hline
\end{tabular}

of the top stream, bottom stream, and the mean flow, respectively. In all the calculations, $U_{m}=1.0$ (i.e., the velocities are normalized by $U_{m}$ ), the density is normalized by the lower ambient density and $p=p_{1}=p_{2}=17.86$; if $\rho=1$, then this choice implies that the sound speed $c=5.0$ and $M_{m}=0.2$.

\section{RESULTS AND DISCUSSIONS}

A typical shear layer contains a wide spectrum of pertubation frequencies and the mixing width of the layer continues to grow with increasing distance. The numerical simulation of one such experiment (Brown and Rosko, 1974) is described here in detail. Additionally, the calculations are continued so as to provide data for a statistical analysis of the mean and fluctuating flowfield quantities of the solution, and these are compared with experimental data. This analysis is repeated for three other simulations - one using the same initial conditions as in the above experiment but with the density ratio inverted (Brown and Roshko, 1974), and two more referring to an experiment (Oster and Wygnanski, 1982) with equal density freestreams. The flow parameters for all four cases are listed in Table 1.

The calculation for Case I began by exciting only the fundamental frequency for 1000 time steps. At this point, the first nine subharmonics $\omega_{2}, \omega_{3}, \ldots, \omega_{10}$ were included in 


\section{Problem 5. Kelvin-Helmholtz Mixing Layers}

the inflow boundary perturbation and retained for the remainder of the calculation. All ten modes were used from the beginning for Cases III and IV. We computed a total of 20,000 time steps for Case I, 30,000 steps for Case III and 26,000 steps for Case IV. The Case II flowfield was initialized using the results of the Case I computation at time $t=2579.0$ at which point the $\operatorname{Tanh}(\mathrm{y})$ profile for the density was inverted and the new calculation commenced. A total of 14,000 time steps were computed for Case II (although $\sim 2000$ steps were required for the influence of the initialization procedure to exit the computational mesh). Each time step took about 4 seconds on the LANL CRAY XMP computer.

The dynamic evolution of the Case I flowfield was discussed in Kuhl et al. (1990) using material interface plots. For brevity, one such plot is shown in Figure $2 \mathrm{a}$. This material interface plot actually represents unconnected dots placed at the marker particle locations. The initial interface contained 5,000 equally spaced markers. This number rapidly increased to the imposed maximum of 75,000 during the course of the calculation, which implies that the length of the interface (insofar as this definition remains meaningful) increased by a factor greater than 15 during the course of the calculation. We note that in the experiment the upper gas was helium and the lower gas was nitrogen; this terminology is used below for convenience.

For comparison with this calculated interface, Figure $2 b$ presents a shadowgraph picture of the helium-nitrogen interface recorded by an experiment (Brown and Roshko, 1974, Fig. 3d). The similarities between the calculated and experimental interface are remarkable. The shape and wavelength of the large-scale structures are quite similar.

Also included in Figure 2 are the corresponding density, vorticity and overpressure contours. The shape of the large structures, as deduced from the density contours,
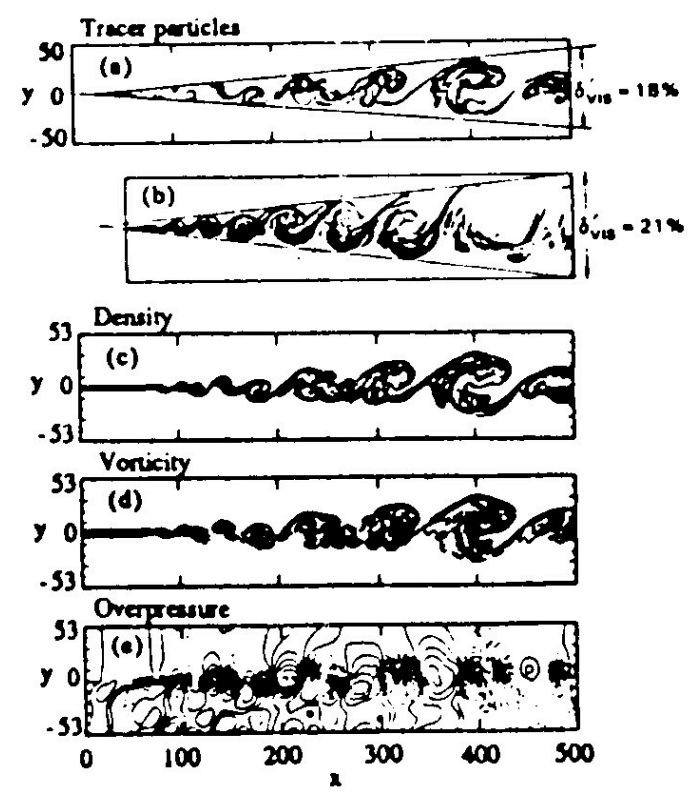

Figure 2 Flowfield for Case I: (a) material interface; (b) shadowgraph from the experiment; (c) density contours; (d) vorticity contours (solid lines denote negative values); and (e) overpressure contours (solid lines denote positive values). 


\section{Problem 5. Kelvin-Helmholtz Mixing Layers}

agrees with the material interface shape, indicating little numerical diffusion. It is interesting to note that vorticity is created near the braid regions and entrained into the structures. Approximately circular low pressure regions are found in each large structure, which generate radial pressure gradients. As the helium-nitrogen interface is entrained obliquely through this pressure gradients, vorticity is generated by the baroclinicmechanism. The maximum value of this vorticity reaches about minus one-half of the inflow vorticity; hence, it must have an effect on the flow. Indeed, close inspection of the material interface reveals some smaller-scale vortices rotating in the counterclockwise direction. This causes the merging patterns to be considerably more complex and less regular than the other cases discussed later.

Figure 3 depicts the corresponding flowfield near the centerline (i.e., $y=0.5$ ). Large fluctuations are evident in the velocity, density and dynamic pressure. In fact, densities
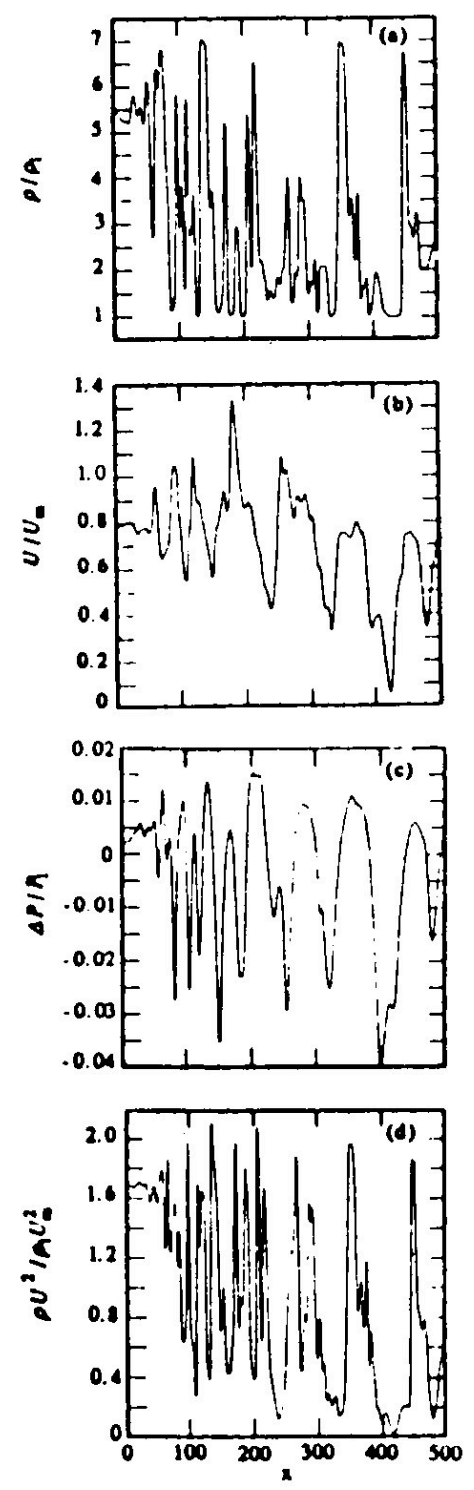

Figure 3 Flowfield along the centerline (i.e., $y=0.5=0.5 \Delta y$ ) corresponding to Figure 2 . 


\section{Problem 5. Kelvin-Helmholtz Mixing Layers}

corresponding to pure helium and to pure nitrogen are seen near the centerline; similarly, the velocity fluctuates between values corresponding to approximately $U_{1}$ and to $U_{2}$. Overpressure fluctuations are small (between +1.5 to -4.0 percent of ambient), which validates the use of the Mach number compromise $(0.29 \leqslant M \leqslant 0.4)$ for this calculation.

To gain some insight into the entrainment process, the density and vorticity contours for Case I are shown in color in Figure 4, where the light fluid is colored blue and the heavy fluid red. In agreement with Grinstein et al.(1986), the density color visualization shows clearly that more high-speed (light) fluid is entrained into the shear layer than low-speed fluid. The vorticity color visualization shows the formation and growth of the large-scale vortical structures more clearly than the contours shown in Figure $2 d$. The baroclinic generation of vorticity (due to density and pressure gradients not being parallel), shown in blue, and the vorticity due to the velocity gradients, shown in red, are vividly seen.

The material interface plots of the first three cases are compared in Figure 5. One notes immediately that the large-scale structures of Case II are considerably less complex than those of Case I. The visual agreement between these plots and the shadowgraphs of the experiments is remarkable. The large-scale dynamics of the Case III equal-density layer is much more straightforward, since relatively little small-scale entrainment and interleaving of the two streams is apparent in the vortical structures. In Figure 6, the calculated visual spreading rates $\delta_{\text {vis }}^{\prime}$ are compared with the experimental data (Brown and Roshko, 1974, Fig. 7). The bars over the calculated results represent the variation in the visual spreading rate over time and the straight lines are suggested by Brown and Roshko(1974). We should note that the straight lines are used here purely as a simple visual guide, and a more involved formula has been proposed by Dimotakis (1989). In Figure 6 we see that the present calculations are in reasonably good agreement with the experimental data. Other measures of the spreading rates based on the mean velocity profiles will be presented later.

In order to implement our statistical analysis, monitoring stations were used to store the flowfield time histories. Each station consists of 82 positions in the fine zone region
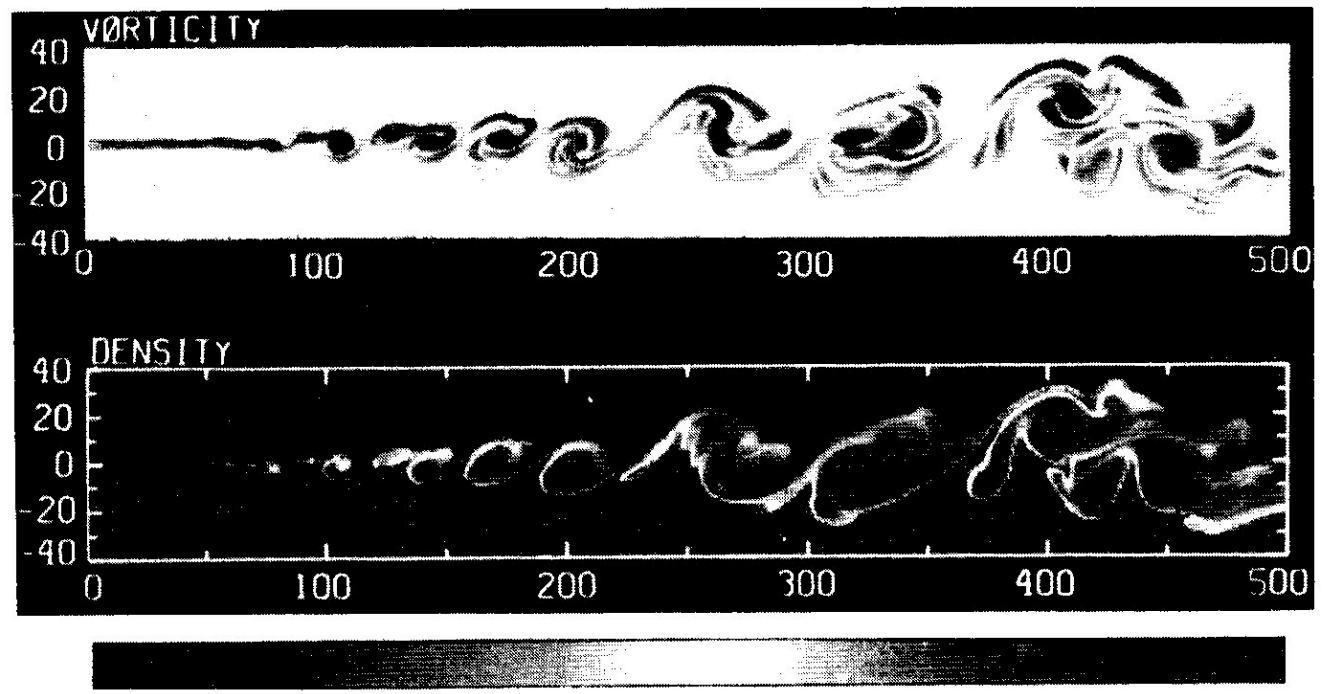

Figure 4 Vorticity and density color visualizations for Case I. See Color Plate III. 


\section{Problem 5. Kelvin-Helmholtz Mixing Layers}
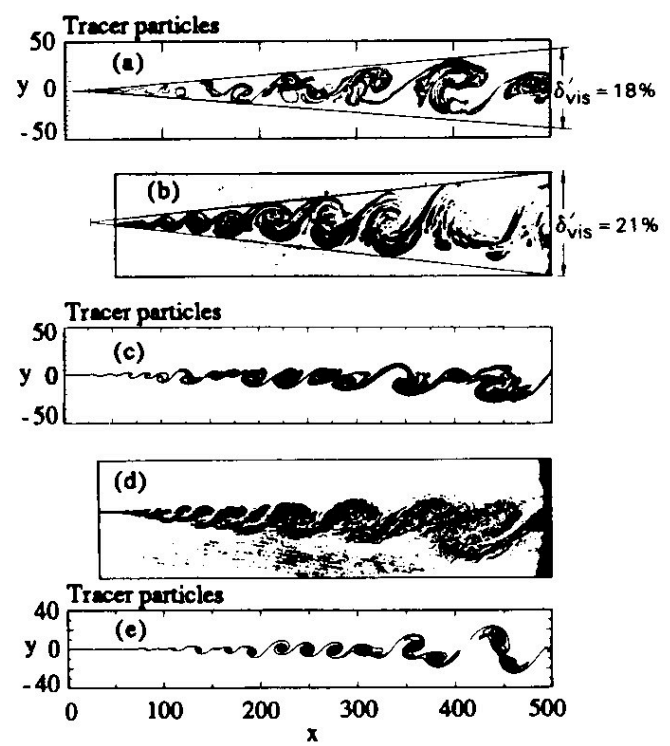

Figure 5 Material interface plots comparing the spreading shear layer cases: (a), (b) Case I, (c), (d) Case II, (e) Case III. Figures (b),(d) are photographs of the experimental record (Brown and Roshko 1974).

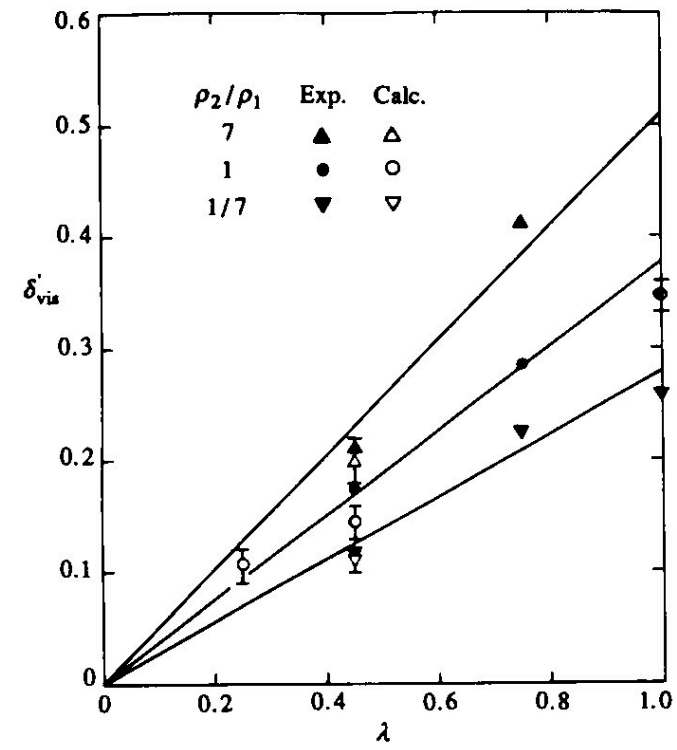

Figure 6 Visual spreading rate, $\dot{\delta}_{\text {vis }}^{\prime}$ vs. $\lambda$, for the spreading shear layer cases.

of the mesh across the shear layer. Except for Case II, nineteen stations located at constant intervals between $x=25$ and $x=475$ were employed. These flowfield histories were then integrated over the last 10,000 time steps to establish the various timeaveraged shear layer profiles. For Case II, three stations located at $x=100,200$ and 400 were employed and only the last 6000 time steps were available at all the 246 positions for the time integration. One reason for not conducting an equally elaborate study for 


\section{Problem 5. Kelvin-Helmholtz Mixing Layers}

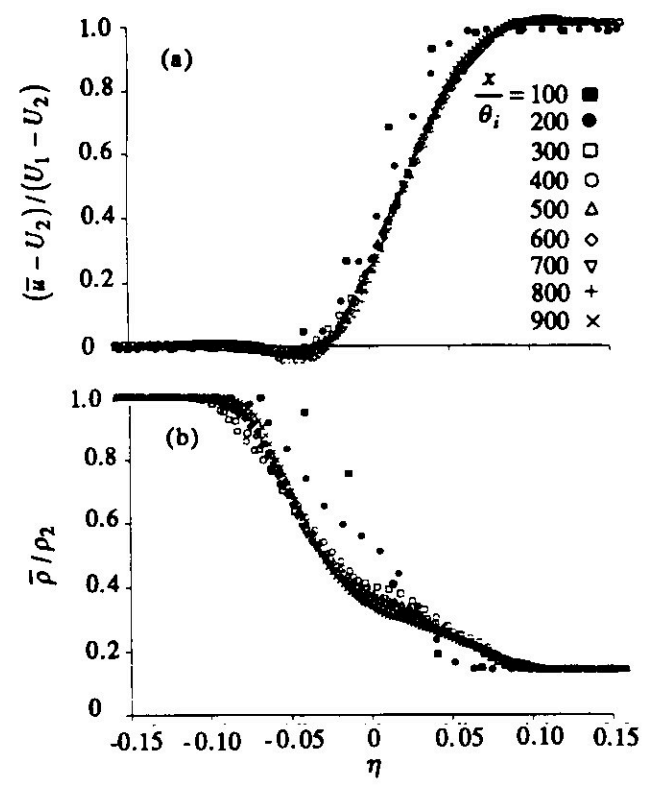

Figure 7 Mean velocity and density profiles for Case I.

Case II is because the mixing is less vigorous than in Case I and hence it is less interesting. In addition, no experimental fluctuating data are available for Case II for comparison; 6000 time steps are adequate to establish mean profiles. Several measures of the spreading rate of the shear layer based on these time-averaged mean velocity profiles have been calculated in the present study. Following previous investigators (Liepmann and Laufer, 1947; Oster and Wygnanski, 1982; Inoue and Leonard, 1987), we introduce the notation $y_{p}$ to denote the location at which the mean streamwise velocity component $\bar{u}$ reaches the value given by

$$
\bar{u}=U_{2}+p\left(U_{1}-U_{2}\right)
$$

Of particular interest are the quantities $y_{0.5}$ and $b\left(\equiv y_{0.95}-y_{0.1}\right)$. Their values at all the 19 stations have been computed for Cases I, III and IV; they exhibit good linear behavior over the last 15 stations. A least-squares linear fit over these data allows us to compute the slope and the corresponding location of the virtual origin $x_{0}$. We have also calculated the momentum thickness (for a constant-density flow)

$$
\theta=\int_{-\infty}^{\infty} \frac{\left(U_{1}-\bar{u}\right)\left(\bar{u}-U_{2}\right)}{\left(U_{1}-U_{2}\right)^{2}} \mathrm{~d} y
$$

for the same cases and obtained the slope and the corresponding $x_{0}$ by the same procedure. The values of $\mathrm{d} b / \mathrm{d} x, \mathrm{~d} \theta / \mathrm{d} x$ and $\mathrm{d} y_{0.5} / \mathrm{d} x$ are listed in Table 2 together with the experimental data of Oster and Wygnanski (1982) and the computed values of Inoue and Leonard (1987). Several conclusions may be drawn from Table 2. 


\section{Problem 5. Kelvin-Helmholtz Mixing Layers}

Table 2 Comparison of various spreading rates

\begin{tabular}{lccccc}
\hline Source & $\lambda$ & $\lambda_{\rho}$ & $\mathrm{d} b / \mathrm{d} x$ & $\mathrm{~d} 0 / \mathrm{d} x$ & $\mathrm{~d} y_{0.5} / \mathrm{d} x$ \\
\hline Oster and Wygnanski & 0.25 & 0 & 0.045 & 0.009 & -0.004 \\
Inoue and Leonard & 0.25 & 0 & 0.049 & 0.010 & -0.006 \\
This study & 0.25 & 0 & 0.050 & 0.010 & -0.006 \\
Oster and Wygnanski* & 0.45 & 0 & 0.084 & 0.016 & -0.018 \\
This study & 0.45 & 0 & 0.081 & 0.016 & -0.016 \\
This study & 0.45 & -0.75 & 0.089 & 0.015 & 0.027 \\
\hline
\end{tabular}

* Interpolated values.

First af all, for constant-density cases, the present finite-difference results agree well with the experimental data and the solution via a vortex method, indicating the reliability of the present approach. Secondly, the growth rate of the shear layer (as measured by either $\mathrm{d} b / \mathrm{d} x$ or $\mathrm{d} \theta / \mathrm{d} x)$ and the slope of its center line $\left(\mathrm{d} y_{0.5} / \mathrm{d} x\right)$ increase in magnitude with increasing $\lambda$. One may note that the growth rate based on visual data (as in Fig. 6) is more sensitive to density variations than $\mathrm{d} b / \mathrm{d} x$. More surprising is the failure of $\mathrm{d} \theta / \mathrm{d} x$ to indicate even the trend of this density effect. For variable-density flows, the momentum thickness should be modified such that

$$
\theta^{\prime}=\int_{-\infty}^{\infty} \frac{\rho\left(U_{1}-\bar{u}\right)\left(\bar{u}-U_{2}\right)}{\rho_{m}\left(U_{1}-U_{2}\right)^{2}} \mathrm{~d} y
$$

and our calculations give $\mathrm{d} \theta^{\prime} / \mathrm{d} x=0.006$ for Case $\mathrm{I}$, a value which is two and a half times smaller than the corresponding value for the constant-density Case IV. This may be explained as follows. As the density of the high-velocity fluid is reduced, the average velocity of this particle and a heavier low-velocity particle will be lower than the average velocity of these two particles at constant density. Therefore, the center line of this shear layer moves toward the high-velocity side relative to the corresponding constant-density flow. For Case I, $\rho_{2}=7 \rho_{1}$, and the density reduction on the highvelocity side is sufficient to yield $\mathrm{d} y_{0.5} / \mathrm{d} x>0$. The region over which the product $\left(U_{1}-\bar{u}\right)\left(\bar{u}-U_{2}\right)$ is the largest occurs now where $\rho / \rho m \ll 1$, therefore producing a smaller value of $\theta^{\prime}$ than the corresponding constant-density case (i.e., $\theta$ ). Since $\theta=\theta^{\prime}=0.5$ at $x=0$, it is easy to see that $\mathrm{d} \theta^{\prime} / \mathrm{d} x<\mathrm{d} \theta / \mathrm{d} x$. Consequently, we conclude that the slope of the momentum thickness is not indicative of the extent of mixing in a variable-density shear layer.

To compare the time-averaged profiles at different stations, a scaling variable

$$
\eta=y /\left(x-x_{0}\right)
$$

will be used, with the virtual origin, $x_{0}$, determined from the least-squares linear fit of $b$ for Cases I, III and IV. Since only three stations are available for Case II, a reliable curve fit cannot be obtained. Therefore, $x_{o}$ determined from the material interface plots is used for that case. The results are: $x_{0}=13.5,-42.0,31.0$ and -2.0 for Cases I, II, III and IV, respectively. 


\section{Problem 5. Kelvin-Helmholtz Mixing Layers}

The development of the mean velocity and density profiles for Case I are shown in Figure 7 . It is clear that the profiles do approach an asymptotic, similar shape as $x$ is increased, and the above scaling helps to collapse the data. In fact, these figures indicate that the profiles at $x / \theta_{i}=300$ are not far from the asymptotic ones $\left(\theta_{i}\right.$ is the initial momentum thickness, $\theta_{i}=0.5$ ). The development of the mean velocity profiles for Cases III and IV is shown in Figures 8 and 9, respectively. It appears that, for these constant-density flows, the above scaling again helps to collapse the data; the mean profiles are somewhat less complicated and the profile at $x / \theta_{i}=200$ is not far from the asymptotic distribution.

The mean velocity and density profiles at selected stations for Cases I, II and III are compared to the mean profiles measured by Brown and Roshko (1974, Figs. 13b and c) and Oster and Wygnanski (1982, Fig. 6d) in Figures 10-12. The calculated profiles are seen to agree reasonably well with the measured profiles. The best agreement between measurements and . calculations is for the simpler, constant-density flow (Case III).

We have also computed the r.m.s. time averages about the mean values. The development of the fluctuating r.m.s. quantities $u^{\prime}, v^{\prime}, \rho^{\prime}$ and shear stress $\left(\overline{-u^{\prime} v^{\prime}}\right)$ for Cases I, III and IV are shown in Figures 13-15. We start by considering the simplest, constant-density, low shear case shown in Figure 13 (Case III). The fluctuating velocity profiles are seen to evolve from low intensities to much higher, well-developed distributions, and the lengths for these fluctuating profiles to reach their asymptotic states are longer than that for the mean velocity profiles. Over the development region, the $u^{\prime}$ profiles sometimes show the double-peaked shape similar to that occurring in the

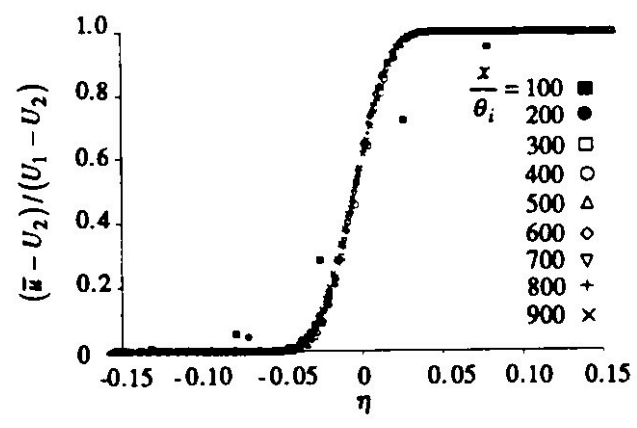

Figure 8 Mean velocity profile for Case III.

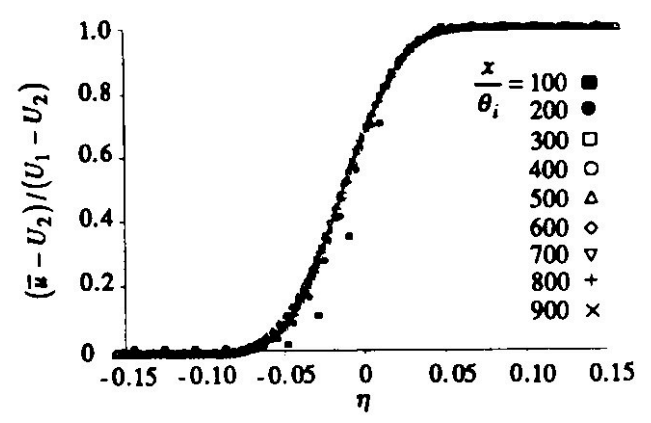

Figure 9 Mean velocity profile for Case IV. 


\section{Problem 5. Kelvin-Helmholtz Mixing Layers}

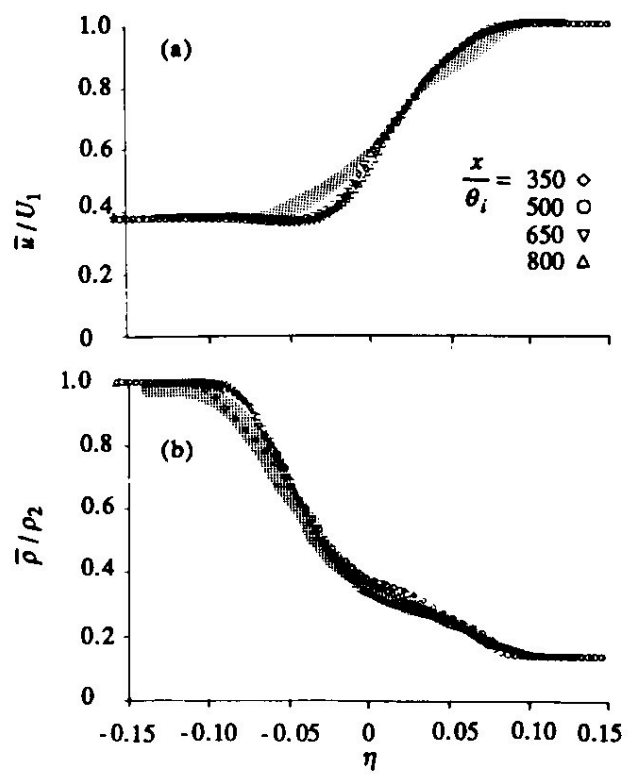

Figure 10 Mean velocity and density profiles for Case I; the shaded regions denote the experimental data band of Brown and Roshko (1974).

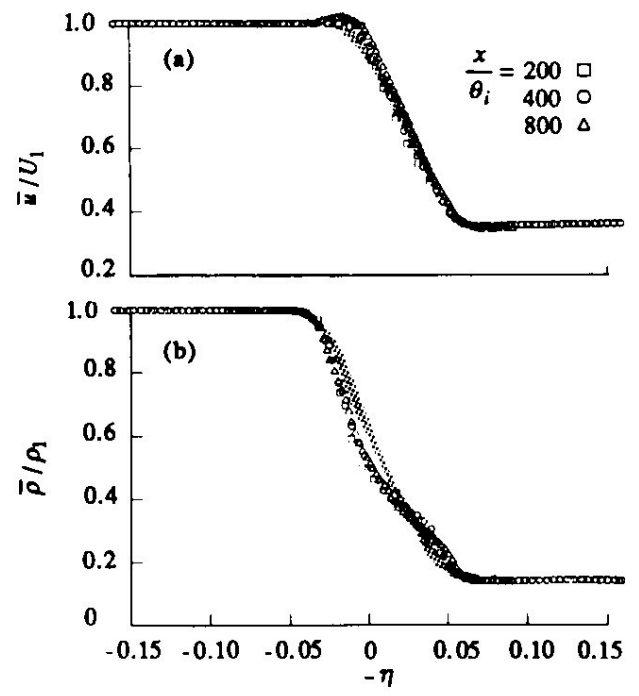

Figure 11 Mean velocity and density profiles for Case II; the shaded regions denote the experimental data band of Brown and Roshko (1974).

Region II of a forced shear layer studied by Oster and Wygnanski (1982, Figs. 20 and 21 ), but the $v^{\prime}$ profiles remain essentially bell-shaped. Note that the development of the shear stress is less orderly than either $u^{\prime}$ or $v^{\prime}$, with perhaps a longer developmental length. Note also that the fluctuating profiles are not as converged as the mean profiles shown in Figure 8.

The corresponding profiles for higher shear (Case IV) are shown in Figure 14. Comparing to the lower shear case, the fluctuating profiles extend to laterally to 


\section{Problem 5. Kelvin-Helmholtz Mixing Layers}

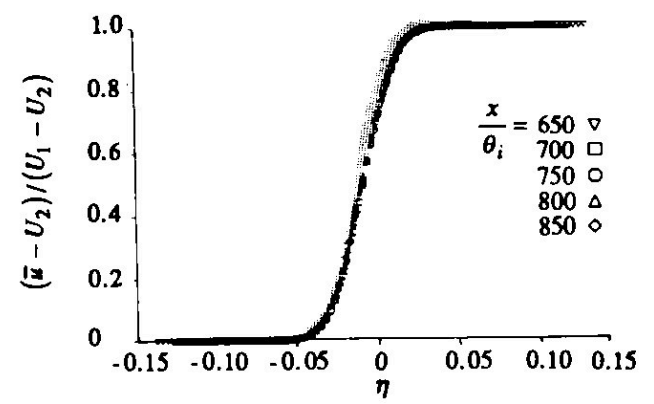

Figure 12 Mean velocity profiles for Case III; the shaded region denotes the experimental data band of Oster and Wygnanski (1982).
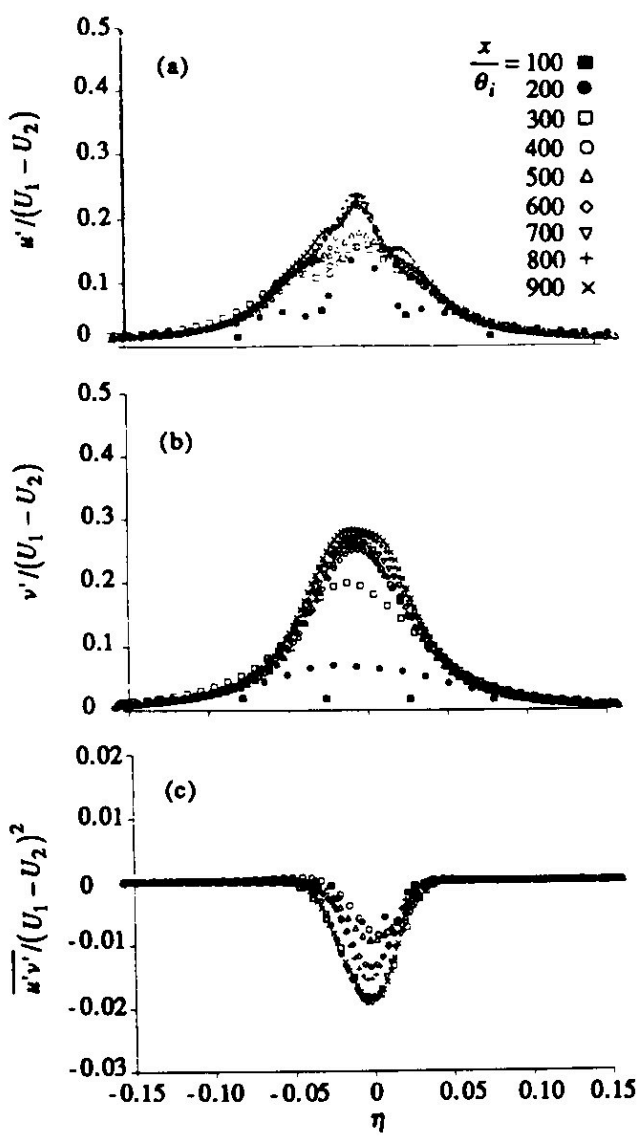

Figure 13 Time-averaged fluctuating-flow profiles for Case III: (a) streamwise velocity; (b) transverse velocity; (c) shear stress.

a greater distance, as was observed experimentally (Oster and Wygnanski, 1982) and, in agreement with stability theory (Monkewitz and Huerre, 1982), the rate of amplification of the first few profiles increases with higher shear. The shear stress distributions converge at an even slower rate, but the overall trends of these two constant-density cases are qualitatively similar. 


\section{Problem 5. Kelvin-Helmholtz Mixing Layers}
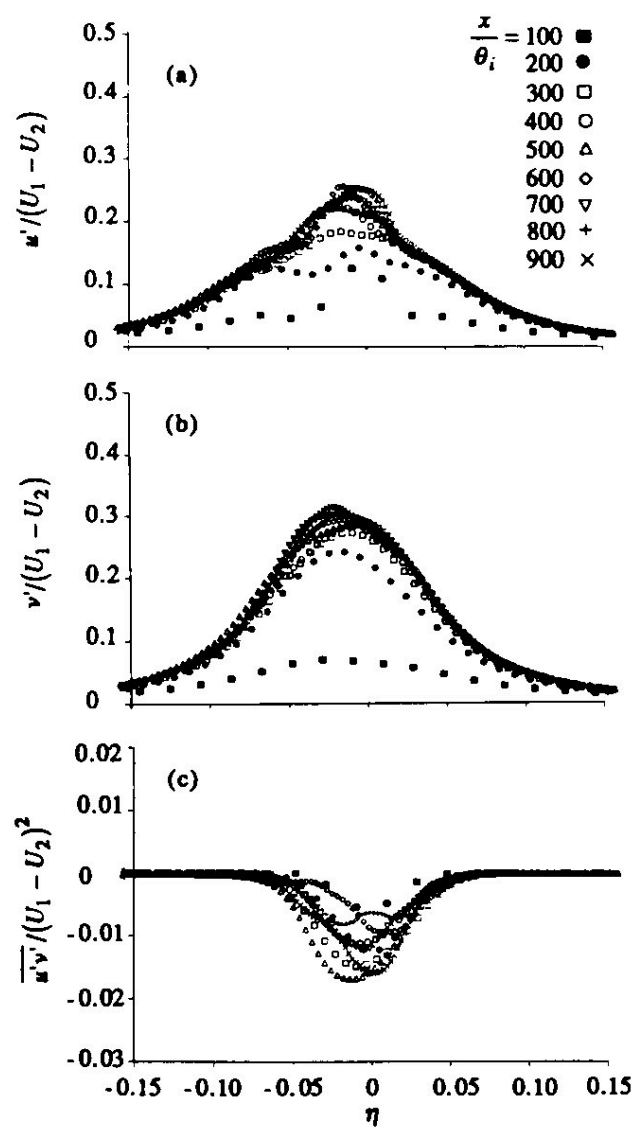

Figure 14 Time-averaged fluctuating-flow profiles for Case IV: (a) streamwise velocity; (b) transverse velocity; (c) shear stress.

When the densities of the two freestreams are not equal, the fluctuating profiles become more complicated. The $u^{\prime}, v^{\prime}$, shear stress and $\rho^{\prime}$ distributions for Case I are shown in Figure 15. Comparing to the corresponding constant-density results shown in Figure 14, we see that $u^{\prime}$ has multiple peaks and appears less converged. The locations of the peak $u^{\prime}$ and $v^{\prime}$ values are now at $\eta>0$, consistent with the result $\mathrm{d} y_{0.5} / \mathrm{d} x>0$ shown in Table 2 . At some stations, the shear stress may become negative $\left(\overline{u^{\prime} v^{\prime}}>0\right)$, suggesting again the type of behavior observed in region II of a forced shear layer (Oster and Wygnanski, 1982, Figs. 25, 26). Since a negative shear stress is not observed in Figure 14, a non-uniform density may also have some effect. The shear stress distributions at the last three stations show multiple peaks and are far from being asymptotic. Unlike the shear stress, the fluctuating density profiles are seen to evolve rather quickly to an asymptotic distribution, with the final, slowest adjustment occurring only near the edges of the shear layer.

The peak values of $u^{\prime}, v^{\prime}, \rho^{\prime}$ and shear stress for Cases I, III and IV are plotted as functions of $x / \theta_{i}$ in Figure 16. They all show a rapid initial growth at small values of $x$. For $u^{\prime}, v^{\prime}$ and $\rho^{\prime}$ this is followed by a more gradual climb to the asymptotic values. There are oscillations in all the results, and they are more pronounced at the peak shear stress 


\section{Problem 5. Kelvin-Helmholtz Mixing Layers}

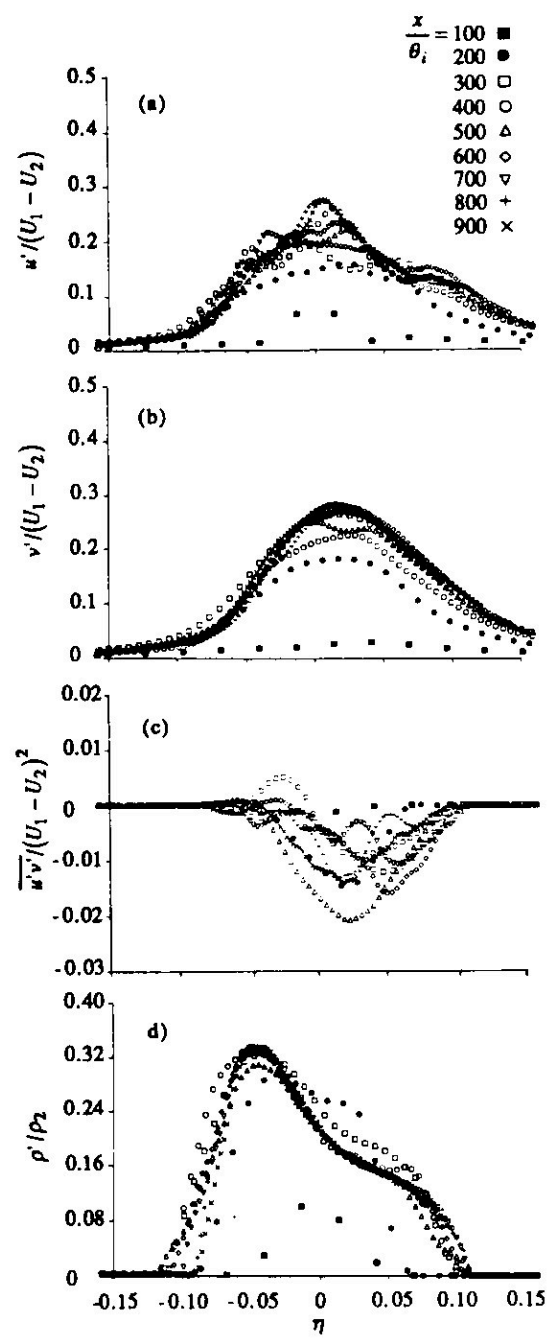

Figure 15 Time-averaged fluctuating-flow profiles for Case I: (a) streamwise velocity; (b) transverse velocity; (c) shear stress; (d) density.

values than in others. If randomness of amplitudes, frequencies or relative phase angles are introduced in the perturbations at the upstream boundary, vortex pairing locations will be less deterministic and these oscillations of the peak values may be reduced. Even though the present mesh distribution is adequate to capture the asymptotic distribution of the mean velocity and density, perhaps a longer fine-zone computational distance in the flow direction is needed to study the asymptotic development of the shear stress distribution, especially for the higher shear, variable density case.

For constant density flows, Oster and Wygnanski (1982) found experimentally that the peak value of $u^{\prime}$ (normalized by the velocity difference across the shear layer as was done here) in the asymptotic region was approximately constant for velocity ratios $\left(r=U_{2} / U_{1}\right)$ between 0.3 and 0.6 . As shown in Figure 16, the weak dependence of the asymptotic peak value on the velocity ratio is predicted here not only for $u^{\prime}$, but also for $v^{\prime}$. In addition, the present calculations show that the peak values of $u^{\prime}$ and $v^{\prime}$ are also insensitive to the density ratio; no measurements are available for comparison. The 


\section{Problem 5. Kelvin-Helmholtz Mixing Layers}
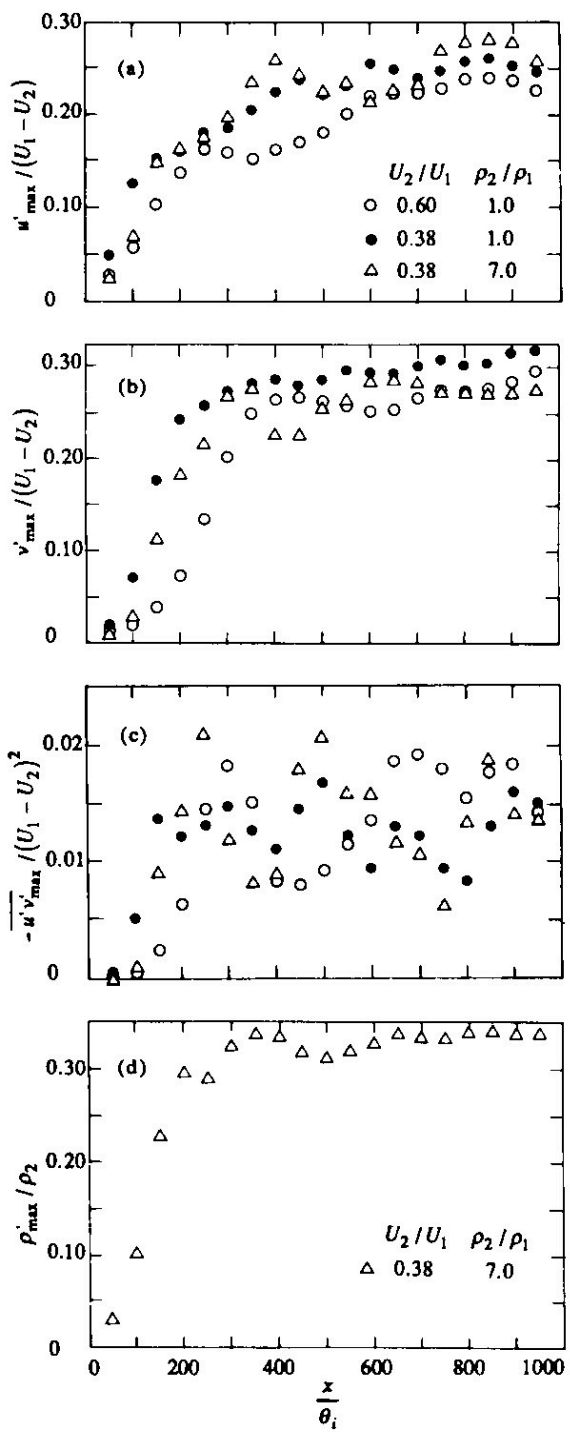

Figure 16 Peak r.m.s. values: (a) streamwise velocity; (b) transverse velocity; (c) shear stress; (d) density.

oscillatory behavior of the peak shear stress, especially for higher shear cases, precludes any definitive conclusion.

The fluctuating velocity profiles at selected stations for Case III are compared in Figure 17 to the measurements of Oster and Wygnanski (1982) and the calculations of Inoue and Leonard (1987). The present $u^{\prime}$ and shear stress results are reasonably close to the data, but the peak value of $v^{\prime}$ is about twice the measured value. It is interesting to note that, in a single-stream shear layer $(r=0)$ with initially laminar condition, Bradshaw (1966) reported a peak $v^{\prime}$ value almost twice its fully-developed asymptotic value. This overshoot was seen to occur in the early region where the flow is presumably more two-dimensional. This observation, together with the close agreement shown in Figure 17 between the present calculations and the two-dimensional results of Inoue and Leonard (1987), based on an entirely different numerical method 


\section{Problem 5. Kelvin-Helmholtz Mixing Layers}
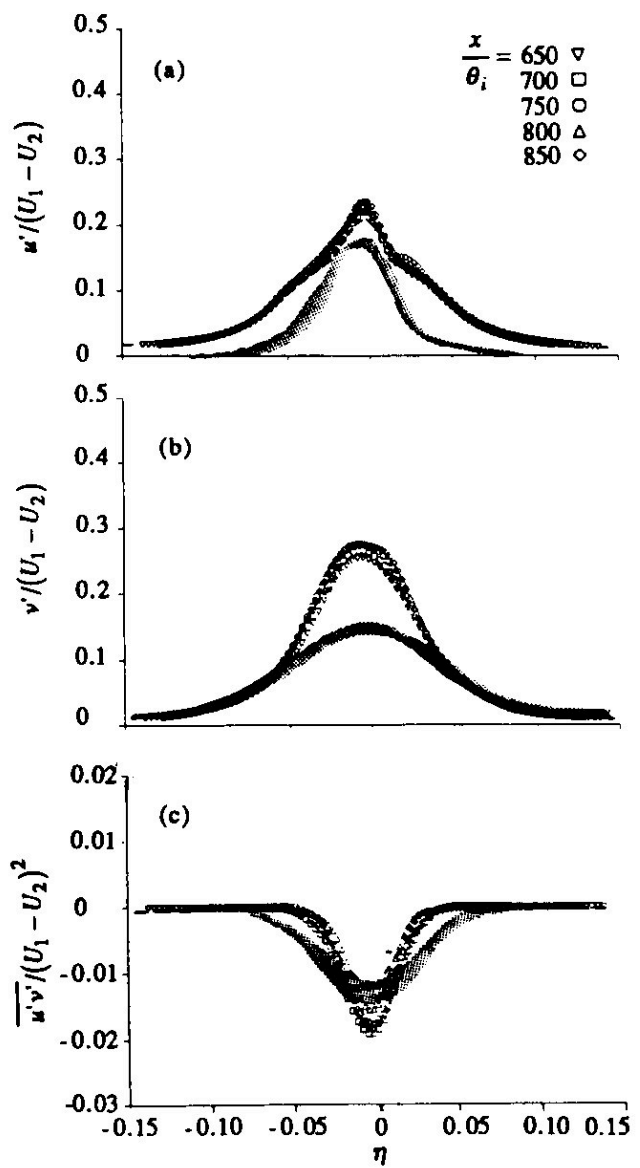

Figure 17 Time-averaged fluctuating-flow profiles for Case III: (a) streamwise velocity; (b) transverse velocity; (c) shear stress. The shaded regions denote the data of Oster and Wygnanski(1982). The dashed lines represent the profiles calculated with a $2 \mathrm{D}$ vortex dynamics code (Inoue and Leonard 1987)

with different initial and boundary conditions, suggests that the large difference between the predicted and measured $v^{\prime}$ profiles may be the result of the two-dimensional flow approximation used in the numerical simulations. Similar conclusions based on forced shear layer studies have been previously reached by Inoue and Leonard (1987) and by Ghoniem and $\mathrm{Ng}$ (1987).

No measurements of the fluctuating velocity profiles for variable density cases are available for comparison. Konrad (1977), however, has measured the density fluctuations for Case I: his data is seen to be in strong agreement with our calculations, shown in Figure 18.

\section{CONCLUSIONS}

Numerical simulations of the dynamics of two-dimensional shear layers have been carried out using an explicit second-order Godunov scheme. Quantitative results have been obtained by statistically averaging the numerical solutions over long time intervals and have been compared with the available experimental data and theoretical 


\section{Problem 5. Kelvin-Helmholtz Mixing Layers}

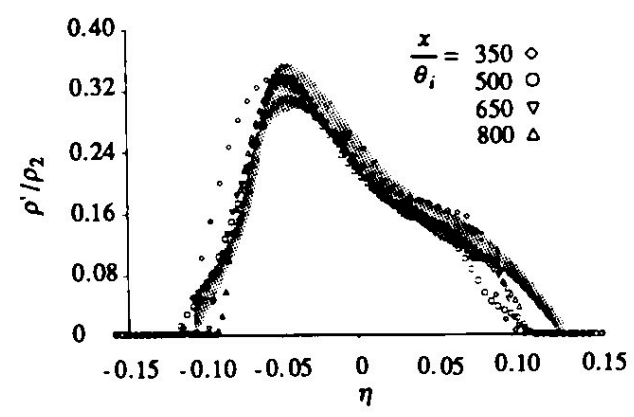

Figure 18 Time-averaged fluctuating density profiles for Case I. The shaded region denotes the experimental data band of Konrad (1977).

calculations. Consistent with the experiments of Brown and Roshko(1974), the present study shows that the spreading rate is higher when the density of the high-speed fluid is reduced relative to that of the low-speed fluid. In addition, the slope of the momentum thickness is not indicative of the extent of mixing in a variable-density shear layer.

Furthermore, this study shows that the mean velocity and density profiles indeed approach an asymptotic, similar shape, and the developmental length is increased when the density of the high-speed fluid is reduced. The time-averaged fluctuating flow profiles approach their asymptotic states more slowly than the mean profiles. Consistent with the experimental finding of Oster and Wygnanski (1982), the peak value of $u^{\prime}$ in the asymptotic region is insensitive to the velocity ratio. In addition, the present calculations show that this is also true for $v^{\prime}$ and the peak values of $u^{\prime}$ and $v^{\prime}$ are also insensitive to the density ratio.

In conclusion, this study demonstrates that present inviscid simulations capture reasonably well the essential features of the flowfield. Therefore the study also suggests that the mean-flow evolution of shear layers is dominated by inviscid effects. Accurate predictions of the mean velocity, mean density, r.m.s. $u^{\prime}$, shear stress and the fluctuating density profiles can be obtained by a two-dimensional flow simulation. However, to improve the accuracy of the r.m.s. $v^{\prime}$ profiles, a three-dimensional calculation is required.

References

Ashurst, W. T. (1979) "Turbulent Shear Flows I”, ed. by F. Durst, et al., Springer-Verlag, Berling, pp. $402-413$.

Bradshaw, P. (1966) "The Effect of Initial Conditions on the Development of a Free Shear Layer", J. Fluid Mech., 26, 225-236.

Brown, G. L. and Roshko, A. (1974) "On Density Effects and Large Structure in Turbulent Mixing Layers", J. Fluid Mech., 64, 775-816.

Chien, K.-Y., Ferguson, R. E., Collins, J. P., Glaz, H. M. and Kuhl, A. L. (1987) “A Study of Mixing in Forced Shear Layers with an Euler Code", AIAA Paper 87-1318.

Chien, K.-Y., Ferguson, R. E., Kuhl, A. L., Glaz, H. M. and Colella, P. (1988) "Inviscid Simulations of Turbulent Shear Layers - Mean Flow Analysis", International Workshop on the Physics of Compressible Turbulent Mixing, Princeton University, Princeton, N.J.

Chien, K.-Y., Ferguson, R. E., Kuhl, A. L., Glaz, H. M., and Colella, P. (1989) "Inviscid Simulations of Turbulent Shear Layers-Fluctuating Flow Profiles", Seventh Symposium on Turbulent Shear Flows, Stanford University, Stanford, CA, 1, 4.3.1-4.3.5.

Chorin, A. J. (1973) "Numerical Studies of Slightly Viscous Flow", J. Fluid Mech. 57, 785-796. 


\section{Problem 5. Kelvin-Helmholtz Mixing Layers}

Chorin, A. J. (1986) "Vortex Methods for the Study of Turbulent Combustion at a Low Mach Number", Tenth Int. Colloq. on the Dynamics of Explosions and Reactive Systems, Prog. in Astronautics and Aeronautics 105, 14-21.

Colella, P. and Glaz, H. M. (1985) "Efficient Solution Algorithms for the Riemann Problem for Real Gases", J. Comp. Phys. 59(2), 264-289.

Colella, P., Ferguson, R. E., Glaz, H. M. and Kuhl, A. L. (1985) "Mach Reflection from an HE-Driven Blast Wave", Dynamics of Explosions, Prog. Astro. and Aero. 106; Bowen, Leyer and Soloukin, editors, 388-421.

Corcos, G. M. and Sherman, F. S. (1984) “The Mixing Layer: Deterministic Models of a Turbulent Flow. Part 1. Introduction and the Two-Dimensional Flow", J. Fluid Mech. 139, 29-65.

Davis, R. W. and Moore, E. F. (1985) "A Numerical Study of Vortex Merging in Mixing Layers", Physics of Fluids 28, 1626-1635.

Dimotakis, P. E. (1989) “Turbulent Free Shear Layer Mixing”, AIAA Paper 89-0262.

Ghoniem, A. F., Chorin, A. J., Oppenheim, A. K. (1982) "Numerical Modeling of Turbulent Flow in a Combustion Tunnel", Philos. Trans. R. Soc. London, Ser. A. 304, 303-325.

Ghoniem, A. F., and Ng, K. K. (1987) "Numerical study of the Dynamics of a Forced Shear Layer", Physics of Fluids, 30, 706-721.

Glaz, H. M., Colella, P., Glass, I. I. and Deschambault, R. L. (1985) “A Numerical Study of Oblique Shock-Wave Reflections with Experimental Comparisons”, Proc. Royal Soc. London, Ser. A. 398, $117-140$.

Glowacki, W. J., Kuhl, A. L., Glaz, H. M. and Ferguson, R. E. (1986) Shock Waves and Shock Tubes, ed. by D. Bershader and R. Hanson, Stanford University Press, Stanford, CA, 187-194.

Grinstein, F. F., Oran, E. S. and Boris, J. P. (1986) "Numerical Simulations of Asymmetric Mixing in Planar Shear Layers", J. Fluid Mech. 165, 201-220.

Ho, C. M. and Huang, L. S. (1982) "Subharmonics and Vortex Merging in Mixing Layers", J. Fluid Mech. $119,443-473$.

Inoue, O. (1985) "Vortex Simulation of a Turbulent Mixing Layer", AI AA Journal 23, 367-373.

Inoue, O. and Leonard, A. (1987) "Vortex Simulation of Forced/Unforced Mixing Layers", AIAA Paper 87-0288; also AIAA Journal 25, 1417-1418.

Konrad, J. H. (1977) An Experimental Investigation of Mixing in Two-Dimensional Turbulent Shear Flows with Applications to Diffusion-Limited Chemical Reactions, Ph.D. Thesis, California Institute of Technology, Pasadena, California.

Kuhl, A. L., Colella, P., Berger, M., Glaz, H. M. and Ferguson, R. E. (1987) "Unstable Wall Jet Evolution for a Double-Mach Stem Flow", Eleventh Int. Colloq. on the Dynamics of Explosions and Reactive Systems.

Kuhl, A. L., Chien, K.-Y., Ferguson, R. E., Glaz, H. M. and Colella, P. (1990) "Inviscid Dynamics of Unstable Shear Layers", DNA-TR-89-6, Defense Nuclear Agency, Alexandria, VA.

Leonard, A. (1980) "Vortex Methods for Flow Simulation", J. Comp. Phys. 37, 289-335.

Liepmann, H. W. and Laufer, J. (1947) “Investigation of Free Turbulent Mixing”, NACA Tech. Note No. 1257.

McInville, R. M., Gatski, T. B. and Hassan, H. A. (1985) "Analysis of Large Vortical Structures in Shear Layers", AIAA Journal 23, 1165-1171.

Michel, F.(1932) "Larm und Resonanzschwingungen im Kraftwerksbetriels (Noise and Resonant Vibrations in Power Plants). Berlin VDI-Verlag.

Monkewitz, P. A. and Huerre, P. (1982) "Influence of the Velocity Ratio on the Spatial Instability of Mixing Layers". Phys. of Fluids 25, 1137-1143.

Oster, D. and Wygnanski, I. (1982) "The Forced Mixing Layer Between Parallel Streams", J. Fluid Mech. $123,91-130$.

Riley, J. J. and Metcalfe, R. W. (1980) "Direct Numerical Simulation of a Perturbed Turbulent Mixing Layer", AIAA Paper 80-0274.

Roshko, A. (1981) "The Plane Mixing Layer: Flow Visualization Results and Three-Dimensional Effects", Lecture Notes in Physics, 136 The Role of Coherent Structures in Modelling Turbulence and Mixing, ed. by J. Jimenez, Springer-Verlag, 208-217.

Soteriou, M. C., Knio, O. M. and Ghoniem, A. F. (1991) "Manipulation of the Growth Rate of a Variable Density, Spatially-Developing Mixing Layer via External Modulations", AIAA Paper 91-0081.

Winant, C. D. and Browand, F. K. (1974) "Vortex Pairing, the Mechanism of Turbulent Mixing Layer Growth at Moderate Reynolds Number", J. Fluid Mech., 63, 237-255.

Woodward, P. R. and Colella, P. (1984) "Numerical Simulation of Two-Dimensional Fluid Flow with Strong Shocks", J. Comp. Phys. 54, 115-173. 


\title{
Problem 6: Wall Mixing Layers
}

\subsection{Dusty Boundary Layer behind a Shock ${ }^{23}$}

\begin{abstract}
A direct numerical simulation was made of the turbulent boundary layer formed by a planar shock propagating along a dusty wall. It differs from previous studies in two respects: (1) it follows the dynamic evolution of the unstable flow without resorting to turbulence modeling; (2) the entrainment of dust into the turbulent boundary layer is included as a part of the computational flow field, thus eliminating the need for a dust scouring model. The calculated mean-flow profiles, boundary layer growth and dust-scouring rate agree with shock tube measurements. In contrast with clean boundary layers, the turbulent fluctuations near the wall were larger than the mean values, as a consequence of the dust mass.
\end{abstract}

\section{Introduction}

Dusty boundary layers are a common feature of shock interactions with realistic surfacesfor example: explosions over soil or ground surfaces, shock wave propagation in coal mines, and industrial dust explosions. Direct theoretical predictions of such flow fields are extremely difficult because of the two-phase turbulent nature of the flow. In addition, wall boundary conditions (e.g.. dust scouring) are particularly intractable. Hence, many of the initial studies $[1,2]$ were empirically based. Hartenbaum [2] measured the mean boundary layer profiles for steady flow over a dust bed, and deduced an empirical dust-scouring rate for flow velocities of 30 to $120 \mathrm{~m} / \mathrm{s}$. More recently, Batt et al. [3] have measured the velocity and density profiles in the turbulent boundary layer behind a shock propagating along a dust bed. However, such nonsteady experiments can give only instantaneous point values at various distances behind the shock; mean-flow profiles and R.M.S. fluctuations cannot be obtained from such data.

Mirels ${ }^{4}$ has published analytic solutions for this problem, but he assumed the boundary layer profiles. Others have performed finite difference simulations: however, they used an empirical mass injection boundary condition on the wall and a $\kappa-\varepsilon$ model of the turbulence $[5,6]$. Since these models were developed for clean flow, and since the dust dramatically modifies the boundary layer, the adequacy of this approach is questionable.

\footnotetext{
${ }^{23}$ Kuhl, A. L., Chien, K.-Y., Ferguson, R. E., Collins, J. P., Glaz, H. M., \& Colella, P. (1989) Simulation of a turbulent dusty boundary layer behind a shock. In Proceedings of the Seventeenth Int. Symposium of Shock Waves and Shock Tubes, Lehigh University, Bethlehem, PA, 17-21 July. Published in Current Topics in Shock Waves (ed. Y. W. Kim), AIP Conference Proceedings 208, American Institute of Physics, New York, NY, pp. 762-769, 1990 (ISBN 0-88318-776-0) Also published as RDA-TR-0263229004-001, R \& D Associates, Los Angeles, CA.
} 


\section{Problem 6. Wall Mixing Layers}

This paper describes a direct numerical simulation of the turbulent boundary layer formed by a planar shock propagating along a dusty wall. It differs from previous studies in two respects: first. it follows the dynamic evolution of the unstable flow without resorting to turbulence modeling; second, the entrainment of dust into the turbulent boundary layer is included as part of the computational flow field, thus eliminating the need for a "dust scouring" model. The formulation of the calculation is described in the next section; this is followed by the results and a discussion that offers suggestions for accurate simulations of such dusty, turbulent flows.

\section{Formulation}

Figure 1 depicts a planar shock propagating with constant velocity $W_{s}$ along a dusty wall. In stationary coordinates (Fig. 1a), a boundary layer is formed behind the shock because of the no-slip condition on the wall. In addition, air percolates into the dust layer, forming a fluidized mixture of dust and air. For simplicity, the mixture was treated as a dense gas of $50 \mathrm{mg} / \mathrm{cc}$, as inferred from experiments [3]. The specific case considered was that of an $M_{s}=1.7$ shock; the gasdynamic states behind the shock (normalized by the ambient conditions, state 1) are listed in Table 1. As in previous analytic studies [7], the analysis was performed in shock-fixed coordinates, (Fig. lb). In this coordinate system, the velocities vary from the value at the edge of the boundary layer, $u_{e}=W_{s}-u_{2}$, to a maximum value on the wall, $u_{w}=W_{s}$.

The computational grid consisted of 500 fine cells in the $x$ direction and 60 fine cells in the $y$ direction; a few coarse zones were used above and to the right of the fine mesh to remove any effects of the computational boundaries. The grid was initialized with the appropriate states from Table 1 and the profiles depicted in Figure lc. The shear layer on the wall was approximated by a $\operatorname{Tanh}(y)$ function with proper asymptotes of $u / a_{1}=1.7$ at $y=0$ and $u / a_{1}=0.77$ at $y=\infty$. The density profile consisted of a three-cell-thick fluidized bed (53 mg/cc) with shocked air above it. Note that the inflection point (IP) of the shear layer was located in the air, three cells above the fluidized bed (FB). The left-hand boundary of the grid was then driven by these same profiles (Fig. lc) with sinusoidal perturbations on the velocity field. Their frequencies corresponded to the frequency of maximum amplification rate from linear stability analysis and its first nine sub-harmonics. The maximum perturbation amplitude was 1 percent. A more complete description of this calculational approach may be found in Kuhl et al. [8].

The dynamic evolution of the flow field was calculated by means of the high-order Godunov scheme of Colella and Glaz [9] that solves the two-dimensional (2-D), non-steady equations of gasdynamics. Since molecular transport effects are neglected, this approach represents a large-Reynolds-number approximation to the dynamics of the flow. (Note that the viscous no-slip condition on the wall is imposed in the in-flow boundary condition of the calculation.) The dust was assumed to be in velocity and temperature equilibrium with the air; thus the entire mixture was modeled as a perfect gas with a constant ratio of specific heats, $\gamma=1.4$. This "dense gas" (DG) model represents an equilibrium approximation that focuses on the inertia effects of the dust. The calculation was run for 6,000 cycles ( $\sim 6$ hours) on a CrayXMP to create a sufficient database for statistical analysis of the flow. 


\section{Problem 6. Wall Mixing Layers}

\section{Results}

Figure 2 depicts the location of the material interface of the fluidized bed. Figure 3 presents the density $(\rho)$, internal energy $(e)$, vorticity $(\omega)$, and pressure $(p)$ contours near the end of the fine-zoned grid. These figures are useful for flow visualization in the boundary layer region. The shear layer in the air rolled up into large rotational structures that entrained material from the fluidized bed. Dense material from the fluidized bed formed long striations because of the flow field of the rotational structures of the turbulent boundary layer. Thus, the dust scouring occurred naturally in the calculation without any modeling.

Figure 4 depicts the boundary layer thickness $\delta$ (i.e., the height of the $99^{\text {th }}$ percentile point of the mean velocity profile) as a function of distance behind the shock. The dusty boundary layer grows linearly with distance: $\delta=0.24 x$, as a result of entrainment and merging of large-scale vortices. Note that this is in contrast to the clean boundary layer on a flat plate, which grows as the 0.8 power of distance from the leading edge: $\delta \propto x^{0.8}$.

The instantaneous flow field at the top of the fluidized bed $\left(y=y_{0}\right)$ is presented in Figure 5. The flow is clearly very chaotic-hence, useful representations of the flow require some type of averaging. To that end, the calculated environment was sampled for all y-cells at stations $x=$ 400. 600. 800, and 950 for each computational cycle. The mean-flow environment was then calculated from these station histories by integrating over the last 5,000 cycles.

The calculated mean density profiles are depicted in Figure 6a. The profiles become thicker with increasing distance from the shock because of turbulent convection. Near the wall all profiles converge to a density of about $\bar{\rho} / \rho_{\infty}=6(\sim 17 \mathrm{mg} / \mathrm{cc})$. This convergence point defines the calculated height of the fluidized bed: $y_{0}=3.5$ cells. Only the flow field above $y_{0}$ was used in the boundary layer profiles; the solution below $y_{0}$ was then considered to be part of the computational modeling of the dust scouring. Note that these calculated profiles are qualitatively similar to the measured dust density profiles [3] shown in Figure 6b.

The mean-flow velocity: $\bar{u} / U_{\infty}$, specific volume: $\bar{\lambda} / \Lambda_{\infty}$, and dynamic pressure: $\bar{q} / Q_{\infty}$ profiles are depicted in Figure 7 (subscript $\infty$ denotes the freestream conditions above the boundary layer). Note that by using the boundary layer scaling $\eta_{B L} \equiv\left(y-y_{0}\right) / \delta$, the calculated profiles collapse to a similarity profile, independent of distance behind the shock. Note also that the calculated profiles are consistent with the LDV and X-ray measurements (shaded regions in Fig. 7) over a soil bed of 10- $\mu m$ diameter loose dust (Batt et al. [3]). Near the wall, densities approach five to six times the free-stream value. This causes the velocities in the wall region to be small $\left(\bar{u} / U_{\infty}<0.1\right)$. Hence, the velocity profile in the wall region seems to be dominated by inertia effects of the dust, and not by fluid viscosity. Note that this situation differs considerably from that of a turbulent boundary layer on a flat plate-where $\bar{u} / U_{\infty} \cong 0.5$ in the law-of-the-wall region, and viscous effects play an important role. Using a control volume analysis of the finegrid region (with a bottom boundary at $y_{0}$ ) and the mean-flow profiles, the non-dimensional mass scouring rate was evaluated as $\dot{m}_{0} / \rho_{\infty} U_{\infty}=0.036$. 


\section{Problem 6. Wall Mixing Layers}

The root-mean-square (R.M.S.) fluctuations about the mean were also evaluated. These fluctuating-flow profiles are presented in Figure 8. The streamwise: $u^{\prime} / U_{\infty}$ and transverse: $v^{\prime} / U_{\infty}$ velocity fluctuations peak at about 30 and 13 percent, respectively, and extend many boundary layer thicknesses from the wall. Thus, dust will be transported well above the mean boundary layer height. The turbulent Reynolds stress: $\overline{u^{\prime} v^{\prime}} / U_{\infty}^{2}$ peaked at about -0.003 , similar to other boundary layers. Density fluctuations: $\rho^{\prime} / \rho_{\infty}$ peaked at about six times the freestream value because of turbulent entrainment of dense material from the fluidized bed. The static: $p^{\prime} / P_{\infty}$ and dynamic $q^{\prime} / Q_{\infty}$ pressure fluctuations peaked at about 17 and 42 percent, respectively.

The local fluctuation-intensity profiles are presented in Figure 9. In constrast with clean boundary layers, turbulent velocity fluctuations were much larger than their mean values (e.g., $u^{\prime} / \bar{u}>>1$ and $v^{\prime} / \bar{v}>>10$ ) because $\bar{u}$ is so small in the wall region. Hence, the fluctuations are the most important component of the flow. This seems to pose severe challenges for both experimentalists and turbulence modelers.

A high-order Godunov scheme has also been developed [10] for non-equilibrium gasdust mixtures. This solves the conservation of mass. momentum and energy for each phase, with drag and heat transfer interactions between phases. A non-equilibrium calculation of the dusty boundary layer behind a shock was run assuming very fine $(0.1 \mu \mathrm{m}$ diameter) dust particles. The non-equilibrium mixture results (NE) are depicted by dashed curves in Figures 7 and 8. Both the mean and the R.M.S. profiles are essentially identical to the dense gas results. Surprisingly then, the dense gas approximation is quite an accurate model for this two-phase flow problem.

\section{Discussion and Conclusions}

Dust lofting behind a shock can be viewed as a two-step process: (i) the formation of a fluidized bed, (ii) followed by entrainment of the dense material from the bed by the rotational structures of the turbulent boundary layer. The principal effect of the dust is to change the velocity of the flow. In the wall region, mean-flow velocities are small because dust densities are large. Also, turbulent velocity fluctuations in the wall region are much larger than the mean values due to this same effect.

The present calculations focus on the two most important physical processes in the turbulent boundary layer, namely, the non-steady velocity field of the large rotational structures, and the inertia effects of the dust. An accurate numerical simulation of these processes allows one to capture the first-order physical effects. For example, the velocity and density profiles, the dust scouring rate, and the boundary layer growth agree with the available data [3]. By inference then, non-equilibrium effects, fluid viscosity and three-dimensionality must have a secondary effect on the mean flow. Nevertheless, experimental data are needed to check the accuracy of the calculated R.M.S fluctuations.

This calculation vividly demonstrates the advantage of working in shock-fixed coordinates where the smooth or laminar solution is steady. By performing a non-steady calculation in these coordinates, one can not only capture the rotational structures of the turbulent flow, but also record time histories at fixed distances behind the shock. These can be used to determine both the mean and fluctuating flow profiles without resorting to turbulence modeling - the main limitation being the 2-D flow approximation. This approach represents a 


\section{Problem 6. Wall Mixing Layers}

significant advance over previous approaches that must assume turbulence properties - which are of course unknown for this dusty boundary layer flow.

\section{REFERENCES}

[1] D. Ausherman. Initial Dust Lofting: Shock Tube Experiments, DNA 3162F, 1973.

[2] B. Hartenbaum, Lofting of Particles by a High-Speed Wind, DNA 2737, 1974.

[3] R. G. Batt, V. A. Kulkarny, H. W. Behrens, H. Rungaldier, Shock-induced Boundary Layer Dust Lofting," Shock Tubes and Waves, H. Gronig, Ed., VCH, Weinheim, Germany, 1988, pp. 209-215 (1988).

[4] H. Mirels. Blowing model for turbulent boundary-layer dust ingestion, AIAA, 22(11), 1984, pp. 1582-1589.

[5] M. R. Denison, Two-layer model of dust lofting (in press).

[6] C. J. Wolf, R. C. Strawn, Boundary layers in surface burst flow fields (in press).

[7] H. Mirels, Boundary Layer Behind a Shock or Thin Expansion Wave Moving into a Stationary Fluid NACA TN 3712, 1956.

[8] A. L. Kuhl, K-Y. Chien, R. E. Ferguson, H. M. Glaz, P. Colella, Inviscid Dynamics of Unstable Shear Layers. RDA-TR-161604-006. R\&D Associates, Marina del Rey, CA, 1988.

[9] P. Colella, H. M. Glaz, Efficient solution algorithms for the Riemann problem for real gases $J$. Compo Phys., 59(2), 1985, pp. 264-289.

[10] J. P. Collins, R. E. Ferguson, K-Y. Chien, A. L. Kuhl, J. Krispin, H. M. Glaz, Simulation of shock-induced dusty gas flows using various models, AIAA Fluid Dynamics Conf. AIAA 9423091994.

Table 1. Flow Field States $\left(M_{s}=1.7, \gamma=1.4\right)$

\begin{tabular}{|l|rccccc|}
\hline & \multicolumn{7}{|c|}{ FLOW FIELD } \\
COORDINATES & $p / p_{1}$ & $\rho / \rho_{1}$ & $e / e_{1}$ & $a / a_{1}$ & $u / a_{1}$ & $u / a$ \\
\hline STATIONARY & & & & & & \\
State 1 & 1 & 1 & 1 & 1 & 0 & 0 \\
State 2 & 3.2 & 2.2 & 1.46 & 1.21 & 0.94 & 0.77 \\
\hline SHOCK-FIXED & & & & & & \\
Edge $(y=\infty)$ & 3.2 & 2.2 & 1.46 & 1.21 & 0.77 & 0.64 \\
FB $\left(0 \leq y<y_{0}\right.$ & 3.2 & 44 & 0.0727 & 0.270 & 1.7 & 6.3 \\
\hline
\end{tabular}




\section{Problem 6. Wall Mixing Layers}

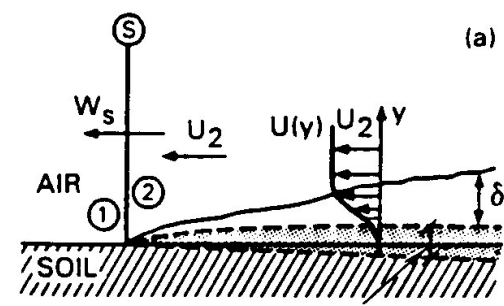

FLUIDIZED BED (FB)

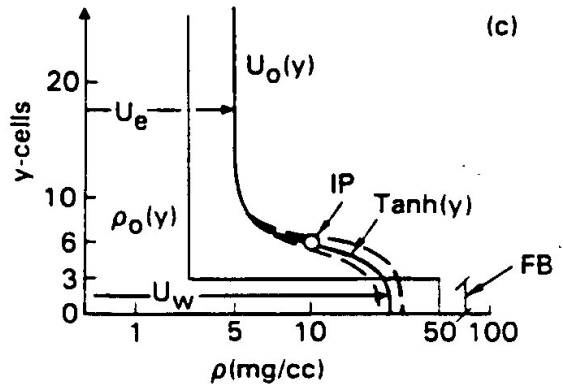

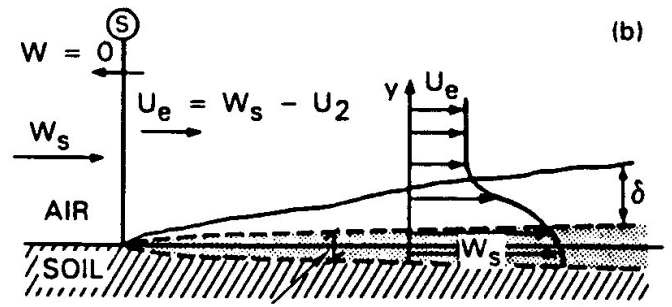

$\mathrm{FB}$

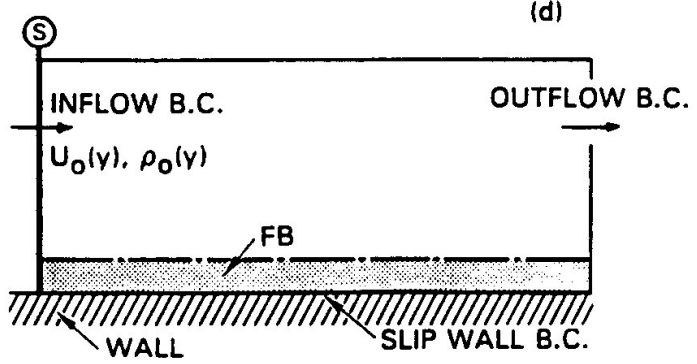

Figure 1. Dusty boundary layer behind a shock: (a) stationary coordinates, (b) shock-fixed coordinates, (c) inflow profiles, (d) grid.
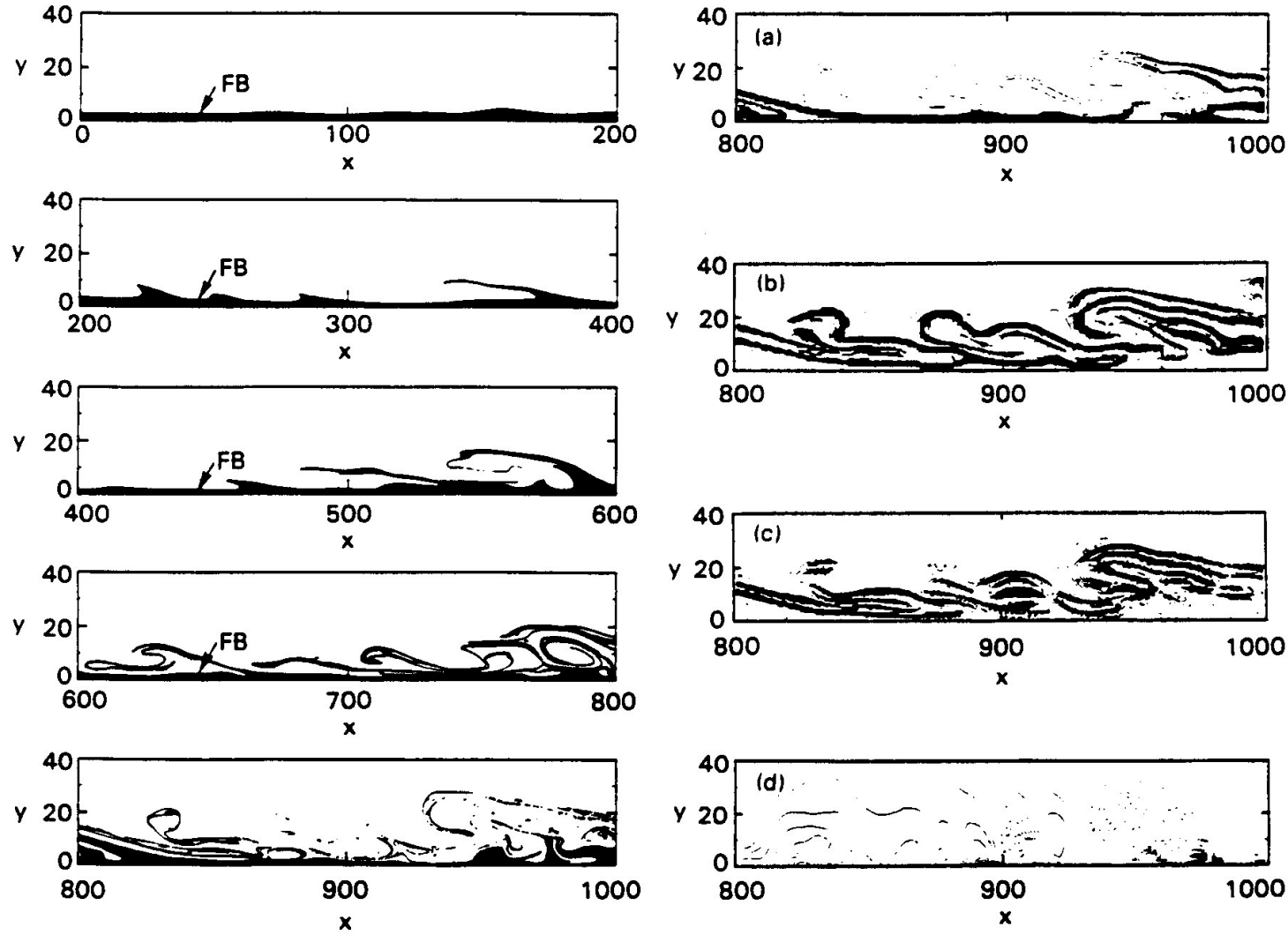

Figure 2. Material interface plots at $t=8202$ showing entrainment of dust from the fluidized bed (FB).

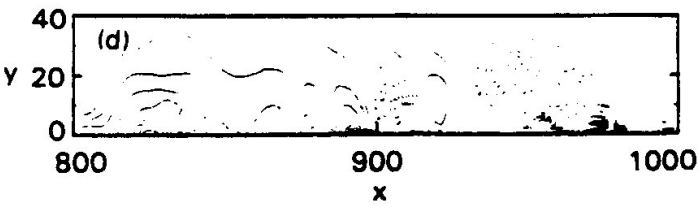

Figure 3. Flowfield contours at $t=8202$

(a) density, (b) internal energy, (c) vorticity, (d) overpressure. 


\section{Problem 6. Wall Mixing Layers}
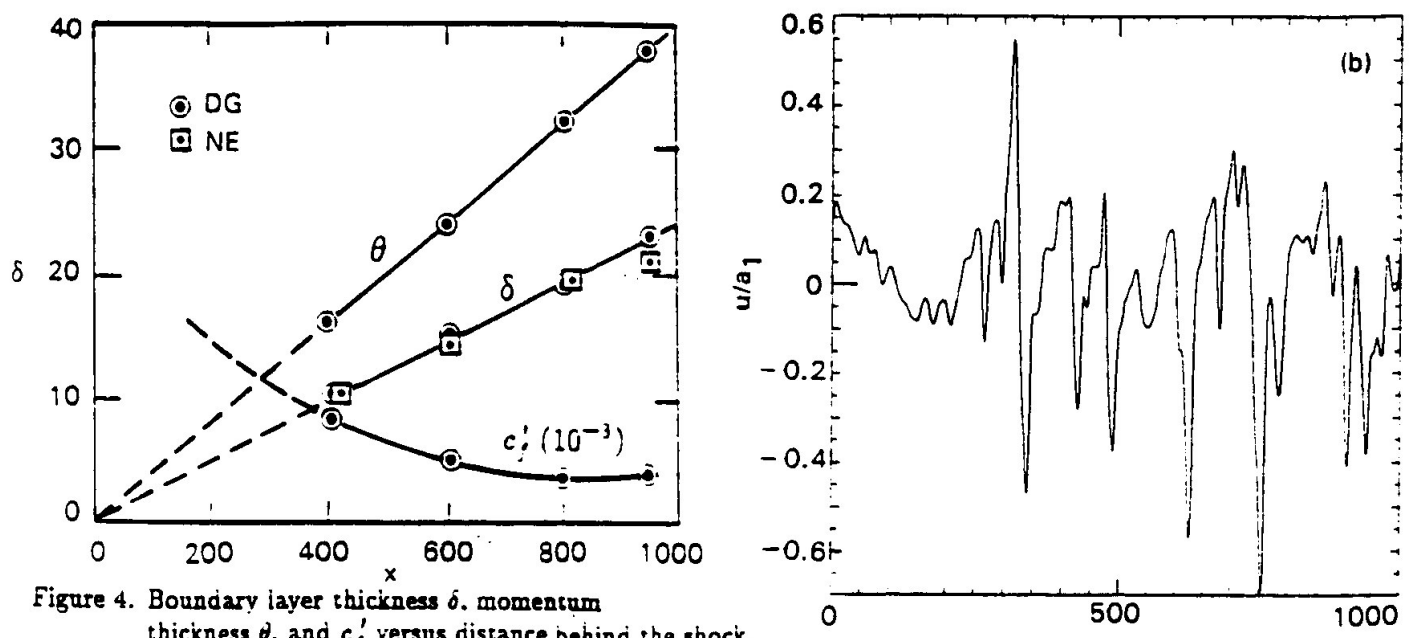

thicicness $\theta$. and $c_{f}^{\prime}$ versus distance behind the shock.
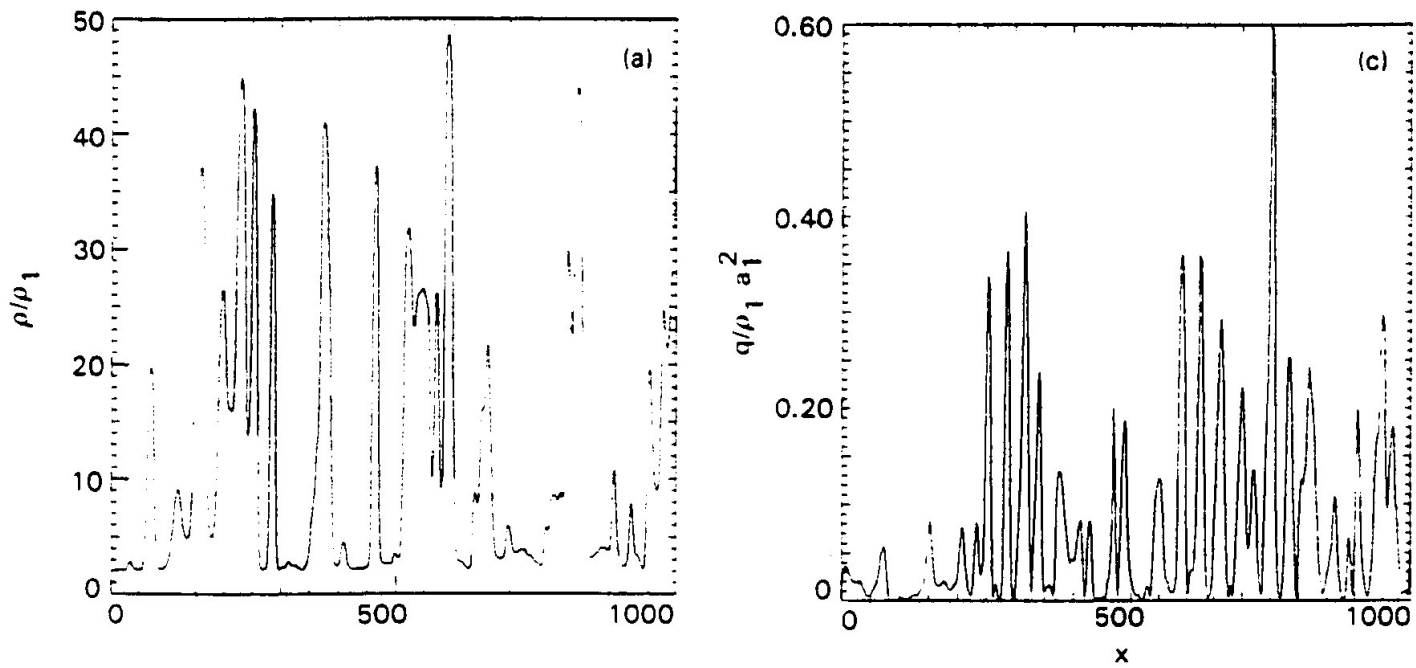

Figure 5. Instantaneous thowitield $(t=8202)$ at the top of the fluidized bed $\left(y=y_{0}\right)$ : $(a)$ density. (b) and (c) velocity and dynamic pressure (stationary coordinates).
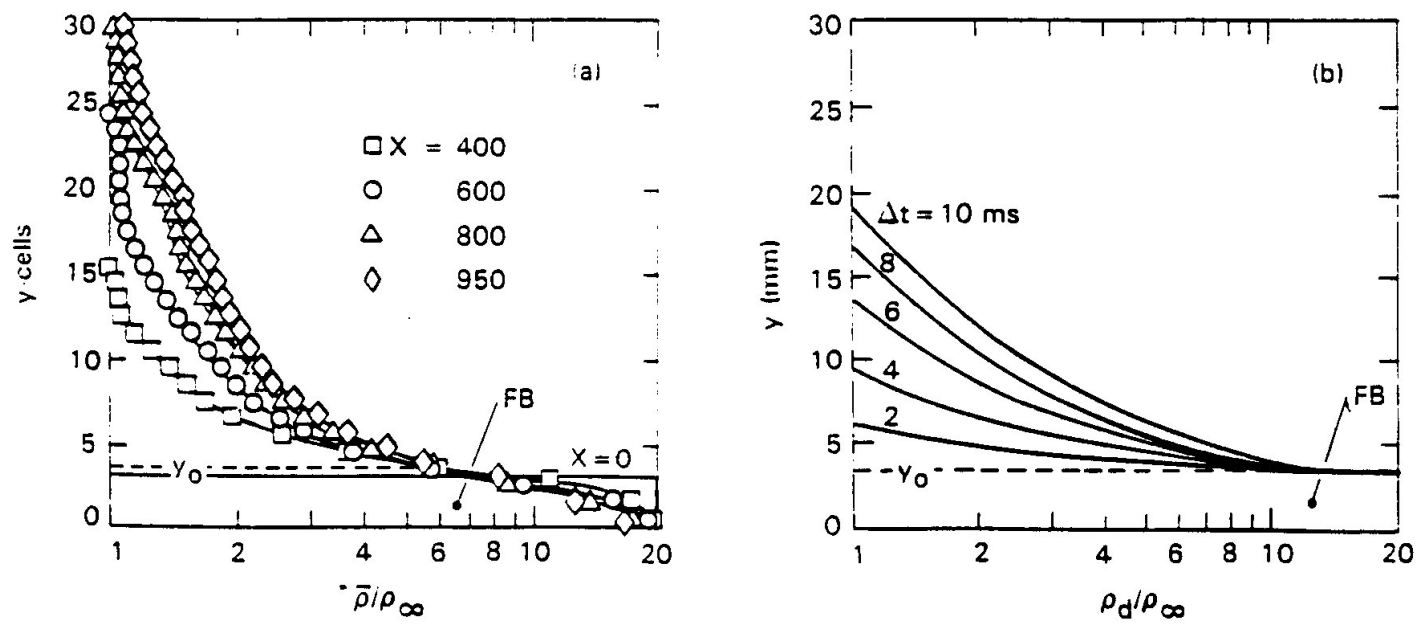

Figure 6. Density protiles behind a $\mathrm{M}_{\mathrm{s}}=1.7$ shock: (a) calculated, (b) measured (Batt et al., 1988). 


\section{Problem 6. Wall Mixing Layers}
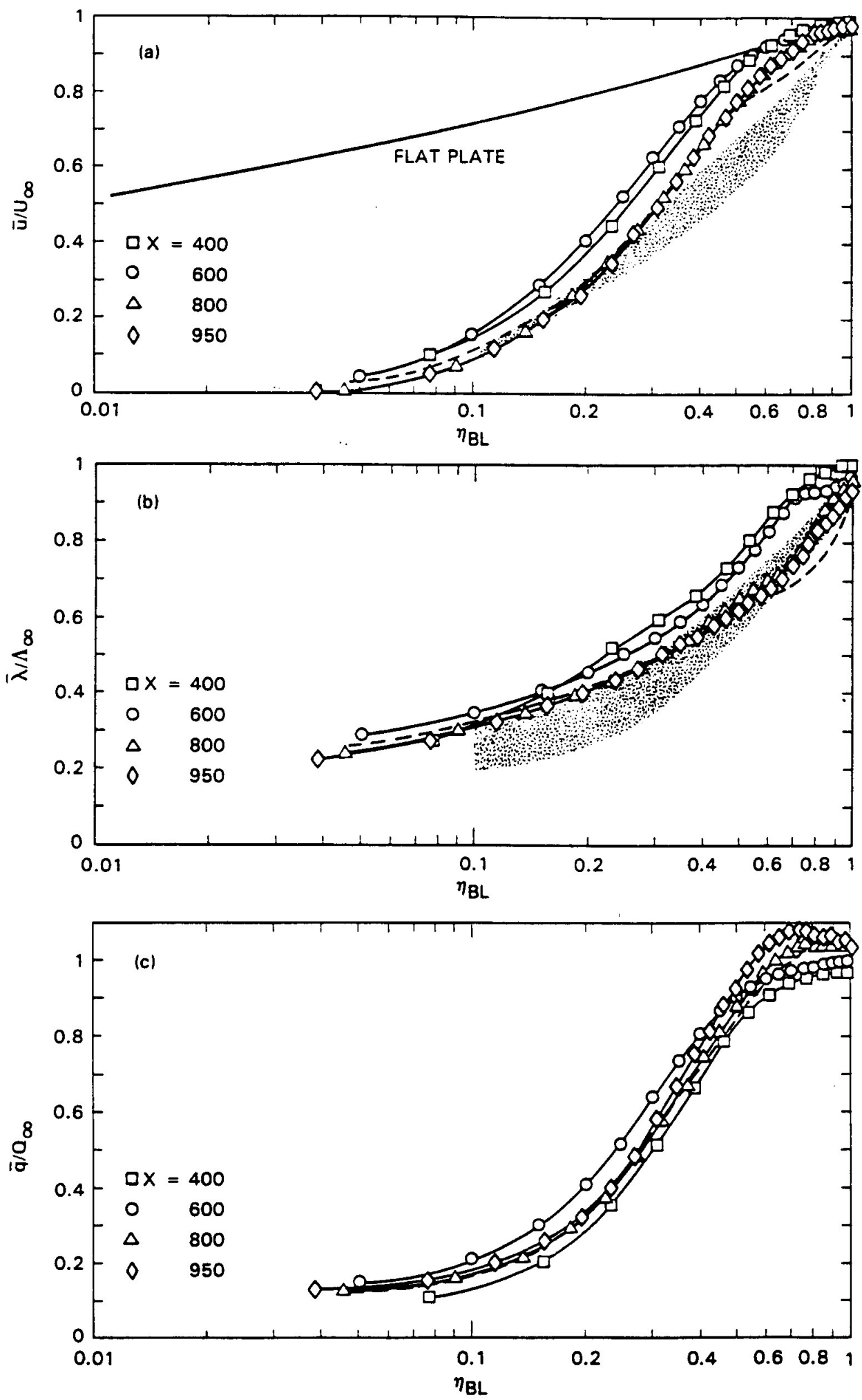

Figure 7. Mean-flow boundary layer profiles: (a) velociry, (b) specific volume, (c) dynamic pressure. Shaded regions denote data bands of Batt et al. (1988). Dashed curve denotes NE mixture results at $x=950$. 
Problem 6. Wall Mixing Layers
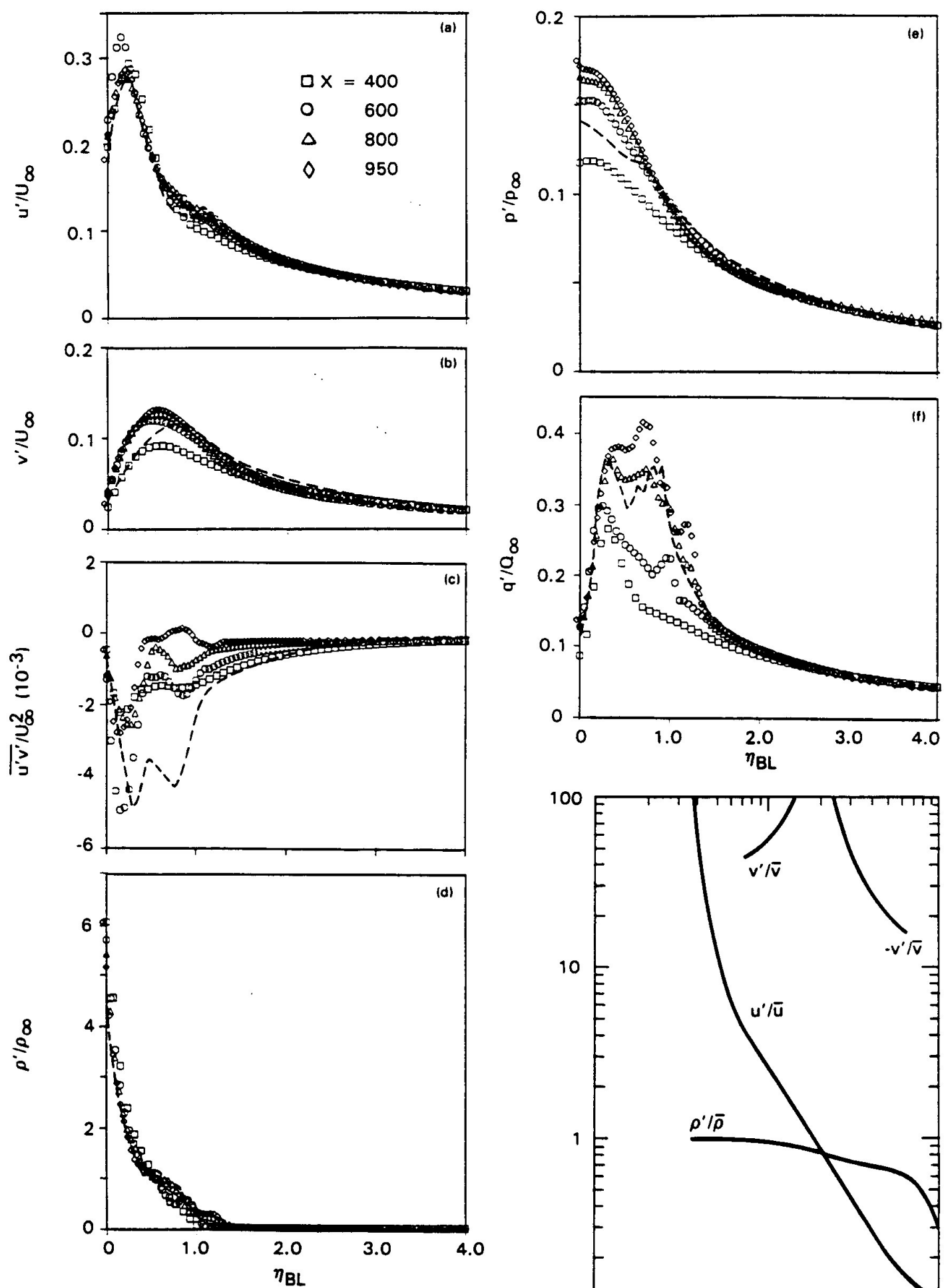

Figure 8. R.M.S. fluctuating-flow profiles: (a) and (b) steamwise and transverse velocities, (c) Reynolds stress. (d) density, (c) pressure, (I) dynamic pressure. Dashed curve shows NE mixture results at $\mathrm{x}=950$.

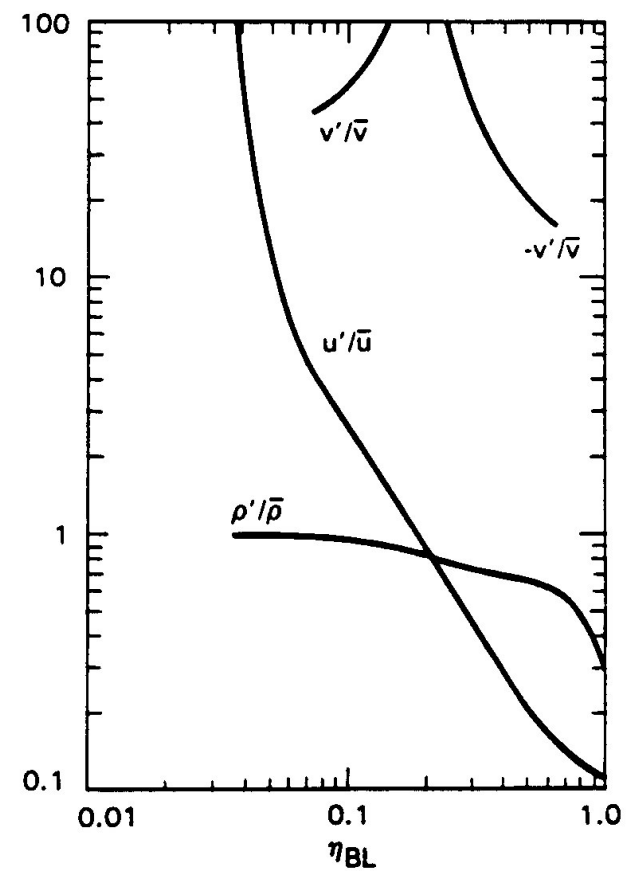

Figure 9. Local fluctuating-intensity profiles at $x=950$. 


\title{
Problem 6. Wall Mixing Layers
}

\subsection{Dense Gas Mixing Layer ${ }^{24}$}

\begin{abstract}
Described here are experiments and calculations of the interaction of a planar shock with a dense-gas layer located on the floor of the shock tube test section. The shock front deposited vorticity in the layer by the baroclinic mechanism. The wall shear layer was unstable and rapidly evolved into a turbulent boundary layer with a wide spectrum of mixing scales. Density effects dominated the dynamics in the wall region.
\end{abstract}

\section{Introduction}

Shock interactions with density discontinuities provide a rich source of fluid-dynamic phenomena. For example, normal shock interactions with planar density interfaces lead to instabilities and turbulent mixing at the interface, as studied by Richtmyer [6] and Meshkov [5]. Planar shock interactions with inclined density interfaces lead to a spectrum of shock refraction effects as shown in the shock tube experiments of Fattah et al. [3], and to the rollup of the interface as demonstrated by the calculations of Zabusky et al. [8]. Planar shock interactions with low density layers located along the wall create shock precursor effects. This leads to a turbulent wall jet as shown in the shock tube experiments of Reichenbach and Kuhl [7]. In these examples, the shock interaction with the interface creates vorticity by a baroclinic mechanism: $\nabla p \times \nabla \rho$. This shear layer is hydrodynamically unstable, and rapidly evolves into a turbulent mixmg region.

Considered here is a new class of such problems, which can be used to study turbulent mixing in boundary layers that are dominated by density effects -in contrast with classical boundary layers that are dominated by viscous effects. In this respect, the current problem can be considered as a boundary layer version of the classic free shear layer experiments by Brown and Roshko [1]. The problem consists of the interaction of a planar sho.ck wave with a Freon layer located along the floor of the test section of a shock tube. Shock interactions with the density interface create a wall shear layer that rapidly rolls up into a turbulent boundary layer. Described here are shock tube experiments and direct numerical simulations of this problem.

\section{Problem Description}

Experiments were performed in the Ernst Mach Institut shock tube. Figure 1 shows a schematic of the test section which had an effective rectangular cross-sectional area of $7.5 \mathrm{~cm}$ by $4 \mathrm{~cm}$. The wall layer was created by means of a fixture that was inserted into the shock tube. It contained a plenum for the injected gas, covered by a $65 \mathrm{~cm}$-long porous ceramic plate (Filtrokelit) that spanned the $4 \mathrm{~cm}$ width of the shock tube. The plenum was filled with Freon-12 at a pressure of $\Delta p_{F} \cong 0.5$ bars and run fore about one minute - to achieve essentially 100 percent Freon concentration in the plenum, and to cleanse the pores of the ceramic plate of

\footnotetext{
${ }^{24}$ Kuhl, A. L., Reichenbach, H. \& Ferguson, R. E., (1991) Shock interactions with a dense-gas wall layer, presented at the Eighteenth Int. Symposium on Shock Waves, 21-26 July 1991, Sendai, Japan. Published in Shock Waves: Proceedings of Eighteenth Int. Symposium on Shock Waves (K. Takayama Ed.) Springer, Berlin pp. 159-166.
} 


\section{Problem 6. Wall Mixing Layers}

residual air. Next, the Freon valve $V_{F}$ was closed and the shock tube was purged of contaminated gases. Then the Freon valve was opened and the plenum was pressurized to $\Delta p_{F}=1.5$ bars for a time $\Delta t_{F}$ (typically 2 seconds) and then the shock tube diaphragm was broken mechanically. To increase the reproducibility of the experiments, all filling procedures were computer controlled.

This procedure created a thin layer $(\sim 0.3 \mathrm{~cm})$ of pure Freon-12 on the floor of the test section. Above this, the Freon concentration decreased gradually in a thicker $(\sim 1.5 \mathrm{~cm})$ diffusion layer. This was caused by residual air pollution in the pores of the ceramic plate, and by bimolecular diffusion processes. The resulting layer was a $2 \mathrm{~cm}$-thick by $65 \mathrm{~cm}$-long laminar layer of Freon whose concentration profile was independent of $x$. The layer could be controlled by the Freon plenum pressure $\Delta p_{F}$ and duration $\Delta t_{F}$; best results were achieved with $\Delta p_{F}=1.5$ bars and $\Delta t_{F}=2$ seconds.

High-speed photography was used as the primary flow field diagnostic - to make visible the turbulent mixing processes occurring in the wall layer. It consisted of 24 sequential frames of shadow-schlieren photographs that were recorded by EMI's Cranz-Schardin camera. In addition, Mach-Zhender interferometry was used to evaluate the pre-shock density profile of the Freon layer.

Figure 2 shows a schematic of the computational domain that was used for numerical simulations of the experiments. A rectangular x-y Cartesian grid of 150 cells by 600 cells was employed. The mesh spacing was uniform with $\Delta x=\Delta y=0.05 \mathrm{~cm}$. The grid was initialized with a quiescent air atmosphere: $\rho_{1}=1.223 \times 10^{-3} \mathrm{~g} / \mathrm{cm}^{3}, p_{1}=1 \mathrm{~atm}$ and $u_{1}=v_{1}=0$. The Freon layer was then modeled by a $\operatorname{Tanh}(y)$ profile, which approached the Freon density on the wall $\rho_{F}=4 \rho_{1}$. The left boundary of the grid was fed with constant conditions corresponding to the state behind a $M_{I}=1.38$ shock wave: $\rho_{2}=1.65 \times \rho_{1}, p_{2}=2.06 \times p_{1}$ and $u_{2}=1.86 \times 10^{4} \mathrm{~cm} / \mathrm{s}$. Outflow boundary conditions were used on the right boundary. The roof and floor were treated as inviscid (slip) walls. Both the air and the Freon were modeled as an ideal gas with $\gamma=1.4$.

\section{Experimental Results}

Figure 3 presents a sequence of photographs showing the interaction of a $M_{I}=1.38$ air shock with a Freon layer. The pre-shock structure of the layer is visible in the first frame. The white band along the floor consists of pure Freon, about $0.3 \mathrm{~cm}$ thick. The black band above it is the aforementioned diffusion layer, where the Freon concentration gradually decreases to zero. The complete layer is about $2 \mathrm{~cm}$ thick in this experiment.

The sound speed in the layer was smaller than that in the air above it, hence the shock propagation was retarded in the layer. This caused the shock front to be curved near the floor. The oblique shock compressed the layer and reflected from the floor as a regular-reflection shock structure. The reflected shock reflected off the layer interface as a rarefaction wave, which stopped the compression of the layer. The rarefaction wave reflected back and forth within the layer, causing periodic accretions and depletions of density. These effects are clearer in the numerical simulation presented in the next section.

By about $10 \mathrm{~cm}$ behind the shock, the wave dynamics effects had damped out, and mixing processes began to dominate. Figure 3 shows that the flow was very unstable, and for 


\section{Problem 6. Wall Mixing Layers}

$\Delta t \geq 0.35 \mathrm{~ms}$, it rapidly evolved into a turbulent mixing layer-even in these small-scale experiments. Although the flow was no doubt three-dimensional, one can occasionally identify large-scale structures (e.g., $\Delta t=0.85 \mathrm{~ms}$ and $0.95 \mathrm{~ms}$ ) that entrain Freon from the wall layer. These structures created density striations that point up and to the right at an angle of about 30 degrees from the floor. Similar striation effects are found in the numerical simulations. One can also observe fine-scale structures, especially near the top of the layer.

\section{Numerical Simulations}

A numerical simulation of the preceding experiment was also performed. As an approximation, it was assumed that the flow was two-dimensional and inviscid. Hence, its dynamics were governed by the mass, momentum and energy conservation laws of gas dynamics. These were solved numerically by the high-order Godunov scheme of Colella and Glaz [2]. The computational setup was shown in Figure 2.

Figure 4 presents contour plots that show the initial shock interaction with the wall layer. The pressure contours show the refraction of the shock front in the layer, the reflection at the floor, and the formation of periodic expansion and compression waves in the layer. The internal energy contours show the periodic compaction $(x=61 \mathrm{~cm}$ and $71 \mathrm{~cm})$ and expansion $(x=66 \mathrm{~cm})$ of the surface. The vorticity contours show that vorticity is generated at the point where the shock interacts with the layer interface. The Freon concentration contours show that the interface begins to rollup almost immediately behind the shock front. At distances greater than about 10 $\mathrm{cm}$ behind the shock ( $<61 \mathrm{~cm}$ in Fig. 4$)$, the rotational structures pair and merge into largerscale structures.

Figure 5 depicts flow field contours at $\Delta t=0.5 \mathrm{~ms}$ after shock passage at $x=70 \mathrm{~cm}$. They show an intense mixing with a spectrum of length scales. As is characteristic of variabledensity flows, the vorticity contours show that counter-sign vorticity (solid lines) are baroclinically generated in the braid region between rotational structures. Peak values are about 0.5 times the peak value of the vorticity of the main flow. The counter-sign vorticity, of course, increases the complexity of the mixing. The tendency in these two-dimensional calculations is to form multiple merging of vortices. At later times, this tendency leads to predominantly largescale structures. In three-dimensional flow, however, vortex stretching and tangling will maintain the fine-scales within the large structures - as is evident in the photographs of Figure 3. Future calculations should include the three-dimensional effects.

The flow field was sampled at station $x=70 \mathrm{~cm}$, and time-averaged profiles in the wall layer were evaluated. The mean flow profiles are presented in Figure 6. Flow variables were non-dimensionalized by the free-stream values, denoted by subscript $\infty$. The profiles are qualitatively similar to our previous simulations of the dusty boundary layer behind a shock [4]. The streamwise velocity profile indicates that the layer remained about $2 \mathrm{~cm}$ thick during this time period-thus eliminating the need for self-similarly stretching the grid to account for growth of the layer. Density effects cause the velocity to decrease to $\bar{u}=0.2 U_{\infty}$ at the floor; if viscous effects were included in the laminar sub-layer, then the velocity would go to zero at $y=0$. In accordance with the negative displacement effect of shock-induced boundary layers, the mean transverse velocities are negative in the layer and reach a peak value of $\bar{v}=-0.035 U_{\infty}$. The mean density reaches a peak value of about $\bar{\rho}=3.8 \rho_{\infty}$ because of the Freon near the floor; hence, this 


\section{Problem 6. Wall Mixing Layers}

mixing layer is strongly influenced by density effects. The mean pressure is essentially constant throughout the layer.

Figure 7 presents the corresponding fluctuating flow profiles. Again, these profiles are qualitatively similar to our dusty boundary layer simulations [4]. The streamwise velocity fluctuations peak at a value of $u^{\prime}=0.31 U_{\infty}$, which is typical of boundary layers. The transverse velocity fluctuations peak at a value of $v^{\prime}=0.26 U_{\infty}$ : this is probably too large by a factor of two - due to the two-dimensional flow approximation. The Reynolds stress $u^{\prime} v^{\prime}$ is positive, indicating that mixing is feeding fluctuating kinetic energy back into the mean flow; peak values reach $\overline{u^{\prime} v^{\prime}}=0.0075 U_{\infty}^{2}$. Density fluctuations are of order 100 percent, due to the Freon. Fluctuations in dynamic pressure and stagnation pressure are also large (e.g., 50 percent) near the floor.

\section{Conclusions}

Shock interactions with a dense-gas layer create a shear layer on the wall by an inviscid (i.e., baroclinic) mechanism. The wall shear layer is unstable, and rapidly rolls up into a threedimensional, turbulent mixing layer with a variety of mixing scales. One of the most interesting features of this problem is that it provides a method for studying high-Reynolds number, turbulent wall layers that are dominated by baroclinically-generated vorticity and density effects - in contrast with classical boundary layers that are dominated by viscous effects. This problem also provides a gasdynamic simulation of turbulent dusty boundary layers if the dust particle diameters are very small. Measurements of both the mean and fluctuating flow profiles of this mixing layer should be performed to check the present numerical predictions.

\section{References}

[1] G. L. Brown, A. Roshko, (1974) On density effects and large structures in turbulent mixing layers, J. Fluid Mech. 64, 1974, pp. 775-816.

[2] P. Colella, H. M. Glaz, (1985) "Efficient solution algorithms for the Riemann problem for real gases, J. Comp. Phys. 59(2) 1985, pp. 264-289.

[3] A. M. Abd-el-Fattah, L. F. Henderson, A. Lozzi, Precursor shock waves at a slow-fast gas interface, J. Fluid Mech. 76(1), 1976, pp. 157-176.

[4] A. L. Kuhl, K.-Y. Chien, R. E. Ferguson, J. P. Collins, H. M. Glaz, P. Colella. Simulation of a turbulent dusty boundary layer behind a shock, Current Topics in Shock Waves, (ed. Y. W. Kim), 1990 American Institute of Physics Press, New York, pp. 762-769.

[5] E. E. Meshkov, Instability of the interface of two gases accelerated by a shock wave, Izv. $A N$ SSSR Mekanika Zhidkosti i Gaza 4(5) 1960, pp. 151-157.

[6] R. D. Richtmyer, Taylor instability in shock acceleration of compressible fluids, Comm. Pure Appl. Math. 13, 1960, pp. 297-319.

[7] H. Reichenbach, A. L. Kuhl, Techniques for creating precursors in shock tubes, $16^{\text {th }}$ Int. Symp. on Shock Tubes and Waves (ed. H. Gronig), VCH press, Weinheim, Germany, 1987, pp. 847-853.

[8] N. Zabusky et al., Vorticity deposition, evolution and mixing for shocked, density-stratified interfaces and bubbles, $18^{\text {th }}$ Int. Symp. on Shock Waves, 1991 (in press). 
Problem 6. Wall Mixing Layers
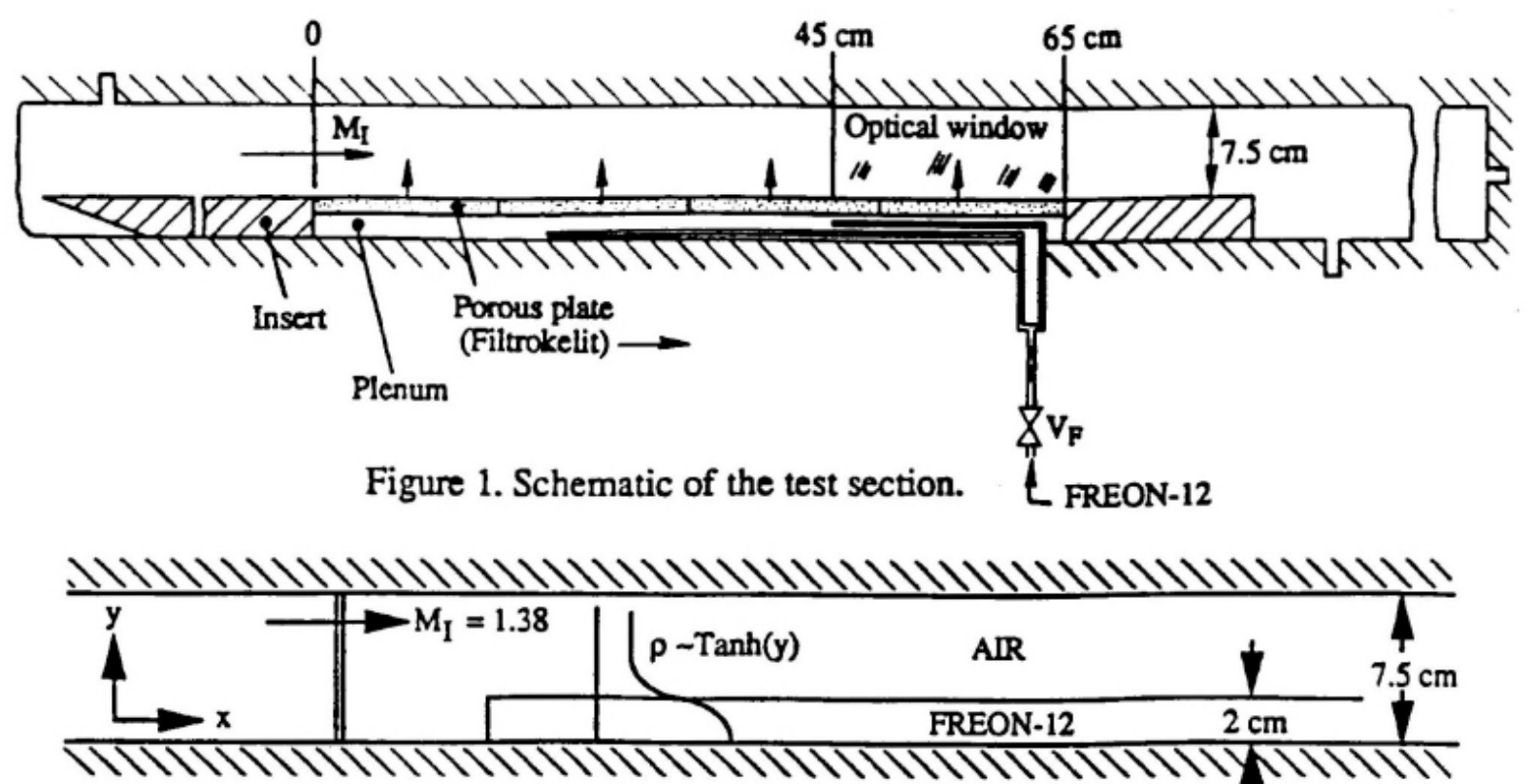

Figure 2. Schematic of the computational domain. 
Problem 6. Wall Mixing Layers

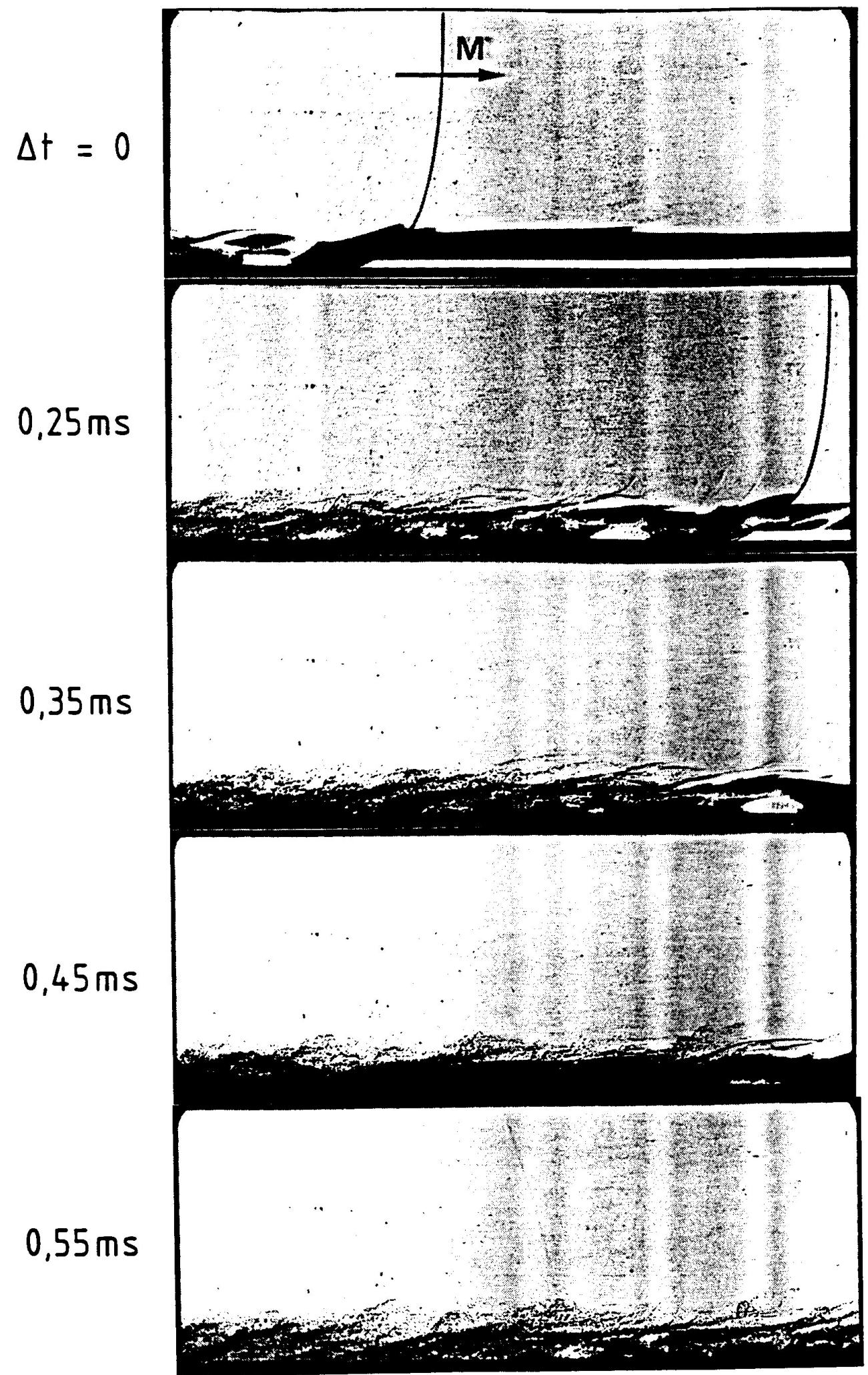

Figure 3. Shadow-schlieren photography of the interaction of a $M_{I}=1.38$ shock wave with a Freon wall layer $\left(\mathrm{pI}_{\mathrm{I}}=1 \mathrm{~atm}\right)$. 
Problem 6. Wall Mixing Layers

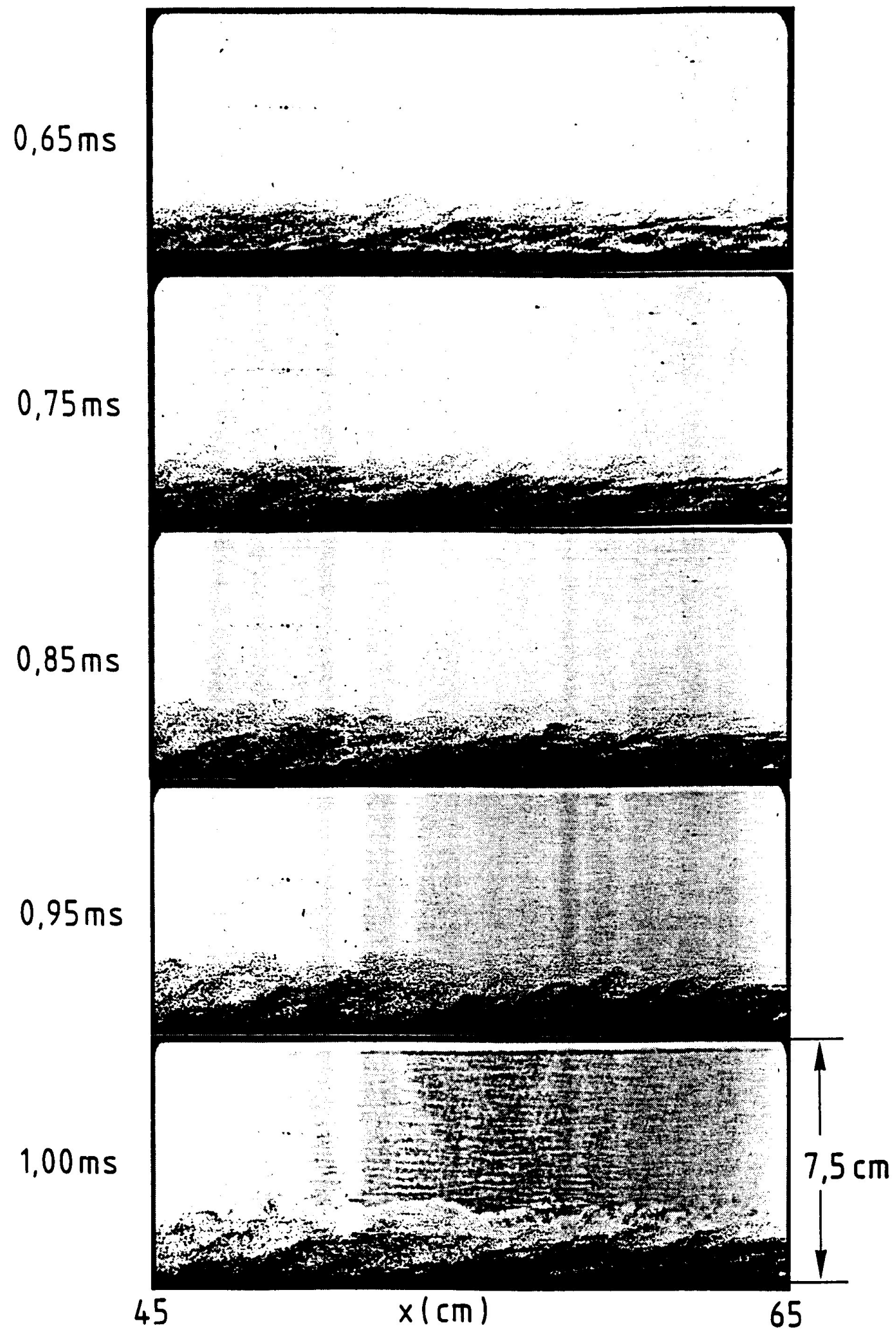

Figure 3. Concluded. 


\section{Problem 6. Wall Mixing Layers}

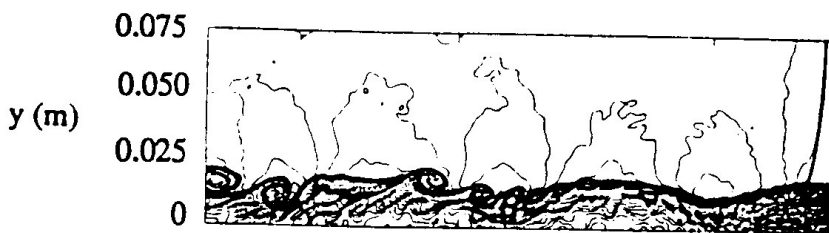

(a) INTERNAL ENERGY

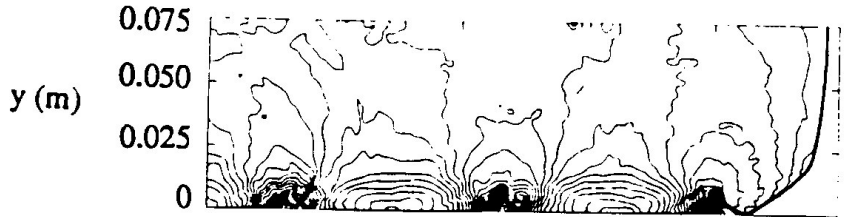

(b) PRESSURE

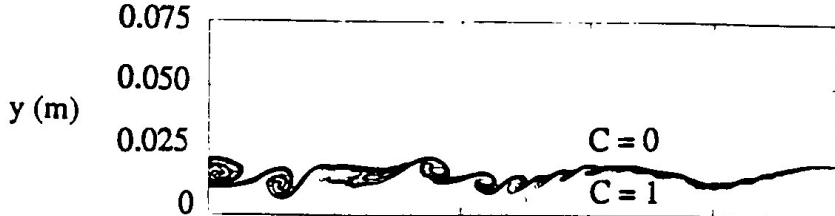

(c) COMP. ZONE

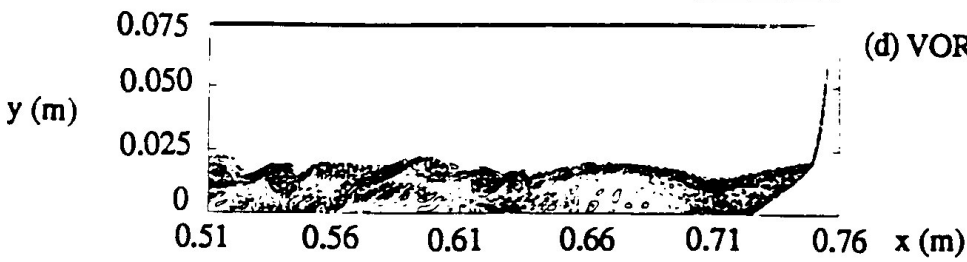

Figure 4. Contour plots showing the initial shock interaction with the wall layer:

(a) internal energy; (b) pressure; (c) Freon concentration; (d) vorticity.
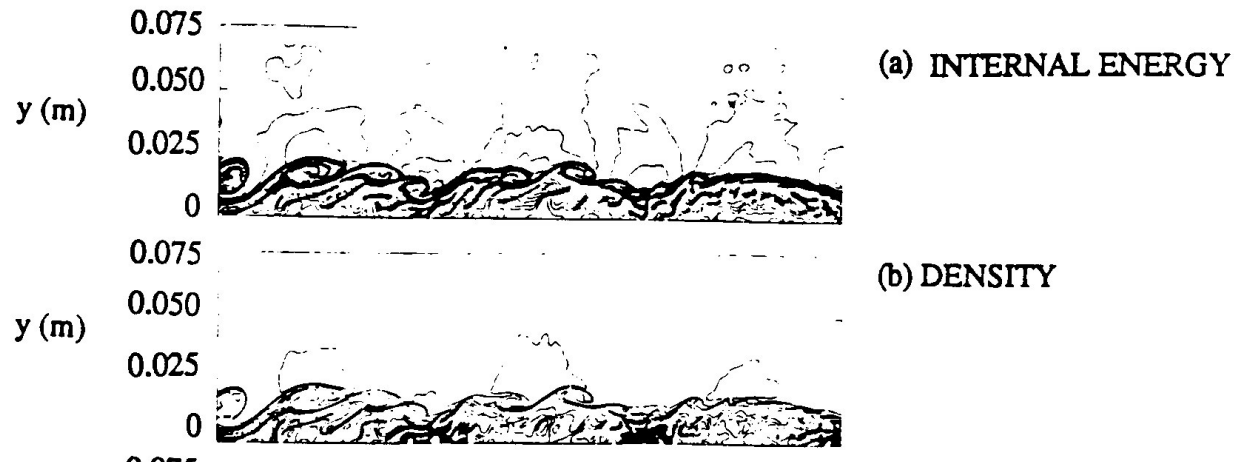

(b) DENSITY

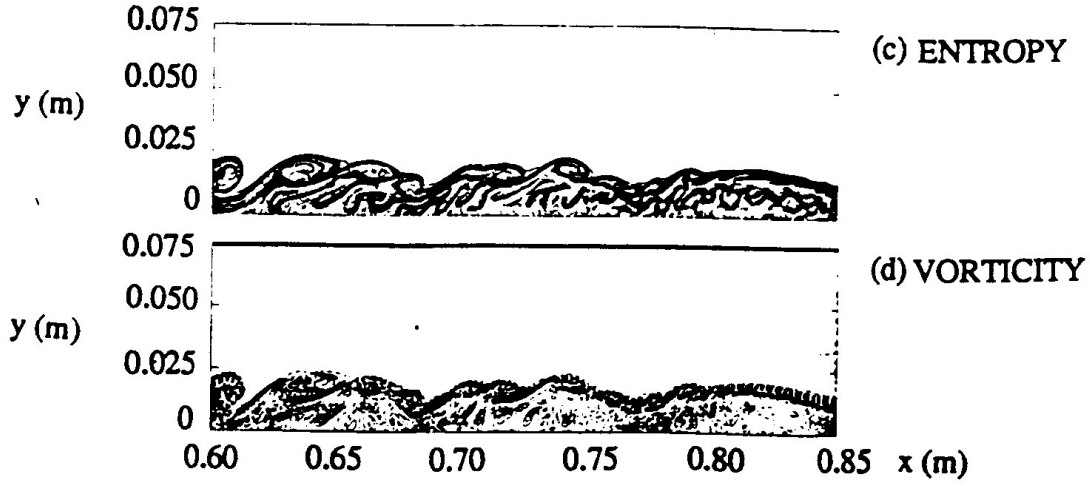

Figure 5. Contour plots showing intermediate-time mixing in the wall layer $(\Delta t=0.5 \mathrm{~ms}$ ): (a) internal energy; (b) density; (c) entropy; (d) vorticity. 


\section{Problem 6. Wall Mixing Layers}
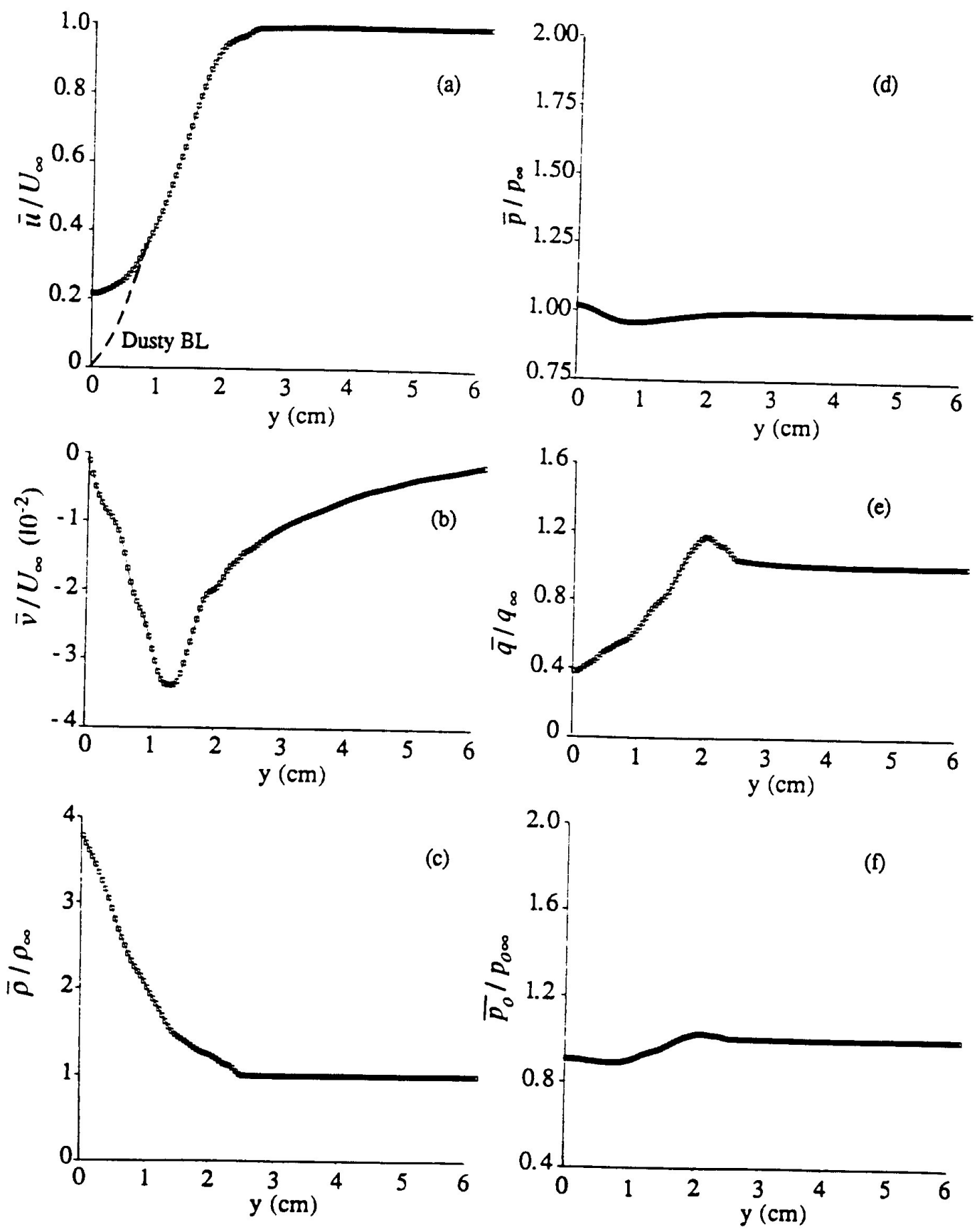

Figure 6. Mean flow profiles in the wall layer $(x=70 \mathrm{~cm})$ : (a) streamwise velocity;

(b) transverse velocity; (c) density; (d) pressure; (e) dynamic pressure; (f) stagnation point. 


\section{Problem 6. Wall Mixing Layers}
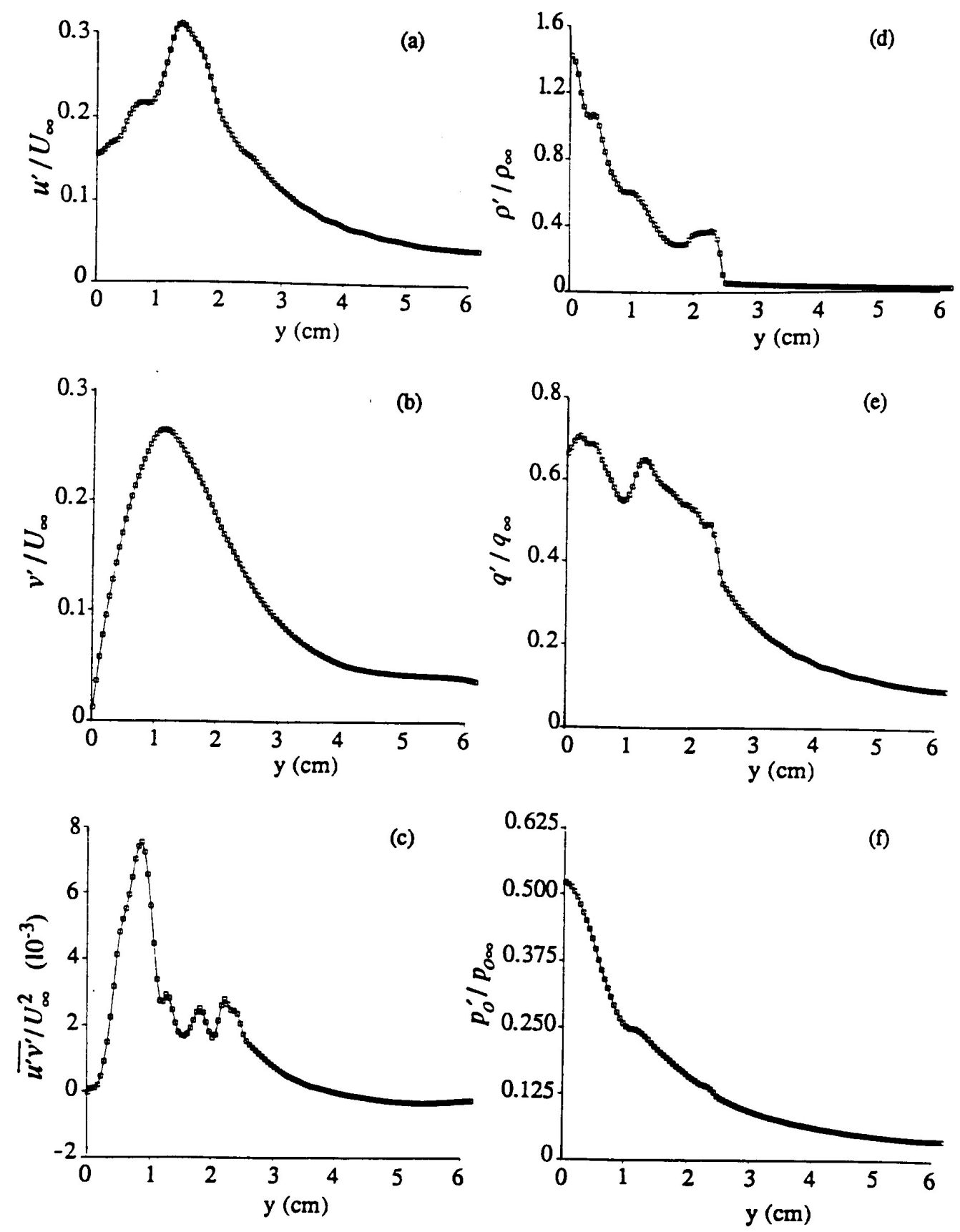

Figure 7. Fluctuating-flow profiles in the wall layer $(x=70 \mathrm{~cm})$ : (a) streamwise velocity; (b) transverse velocity; (c) Reynolds stress; (d) density; (e) dynamic pressure;

(f) stagnation pressure. 


\section{Height of Burst Curves}

\subsection{HOB curves from 1-g Nitropenta charges ${ }^{25}$}

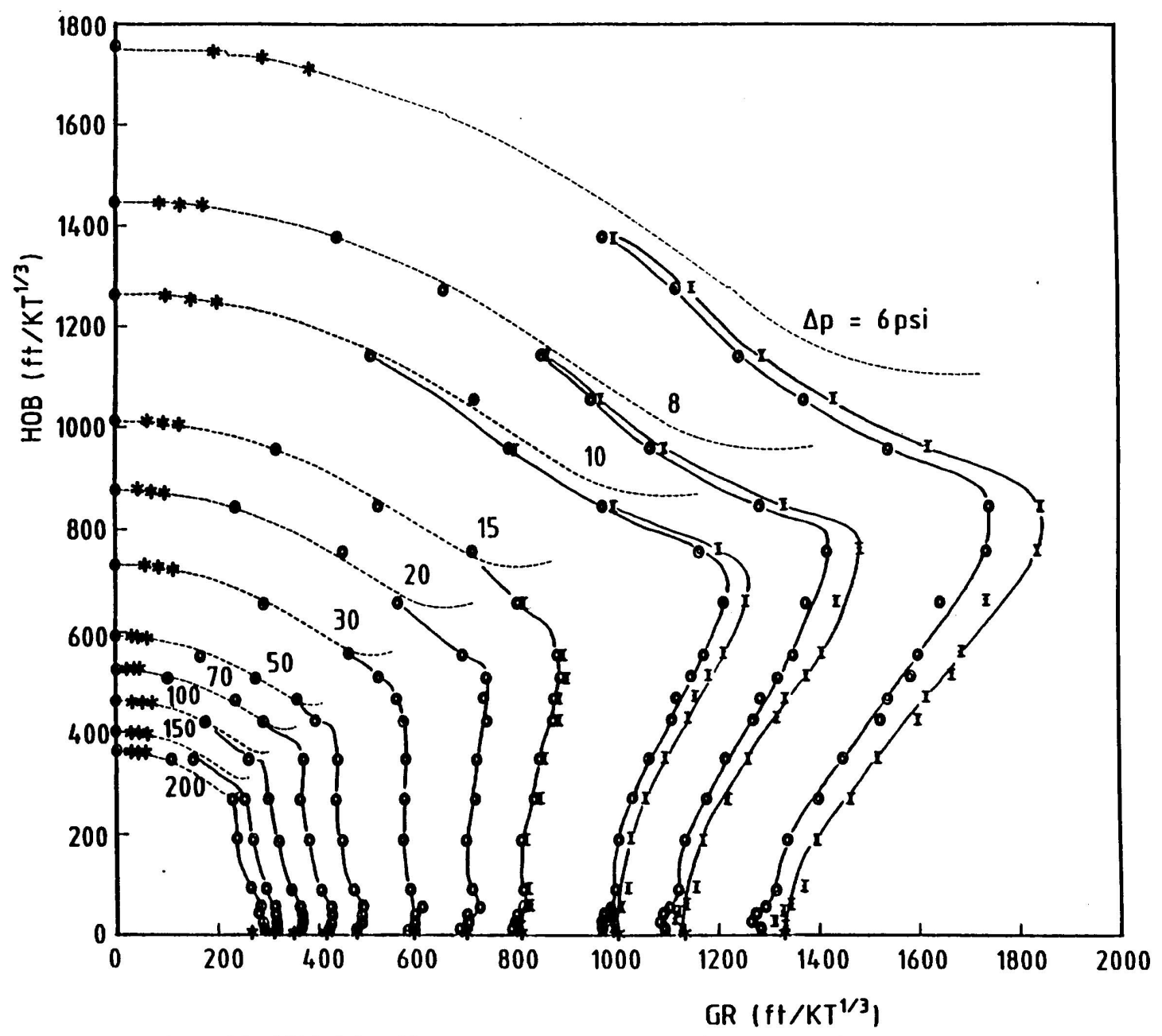

Figure 24. NP height-of-burst curves for a smooth surface (symbol $O$ ) and an ideal surface (symbol I) scaled to a 1 -KT point explosion by function SF for $\Delta \mathrm{p}=6$ to $200 \mathrm{psi}$.

${ }^{25}$ Reichenbach, H. \& Kuhl, A. (1991) HOB curves for 0.5-g NP charges, DNA-TR-90-223, Defense Nuclear Agency, Alexandria, VA, 38 pp. 


\subsection{HOB curves from 8-lb PBX9404 charges $^{26}$}

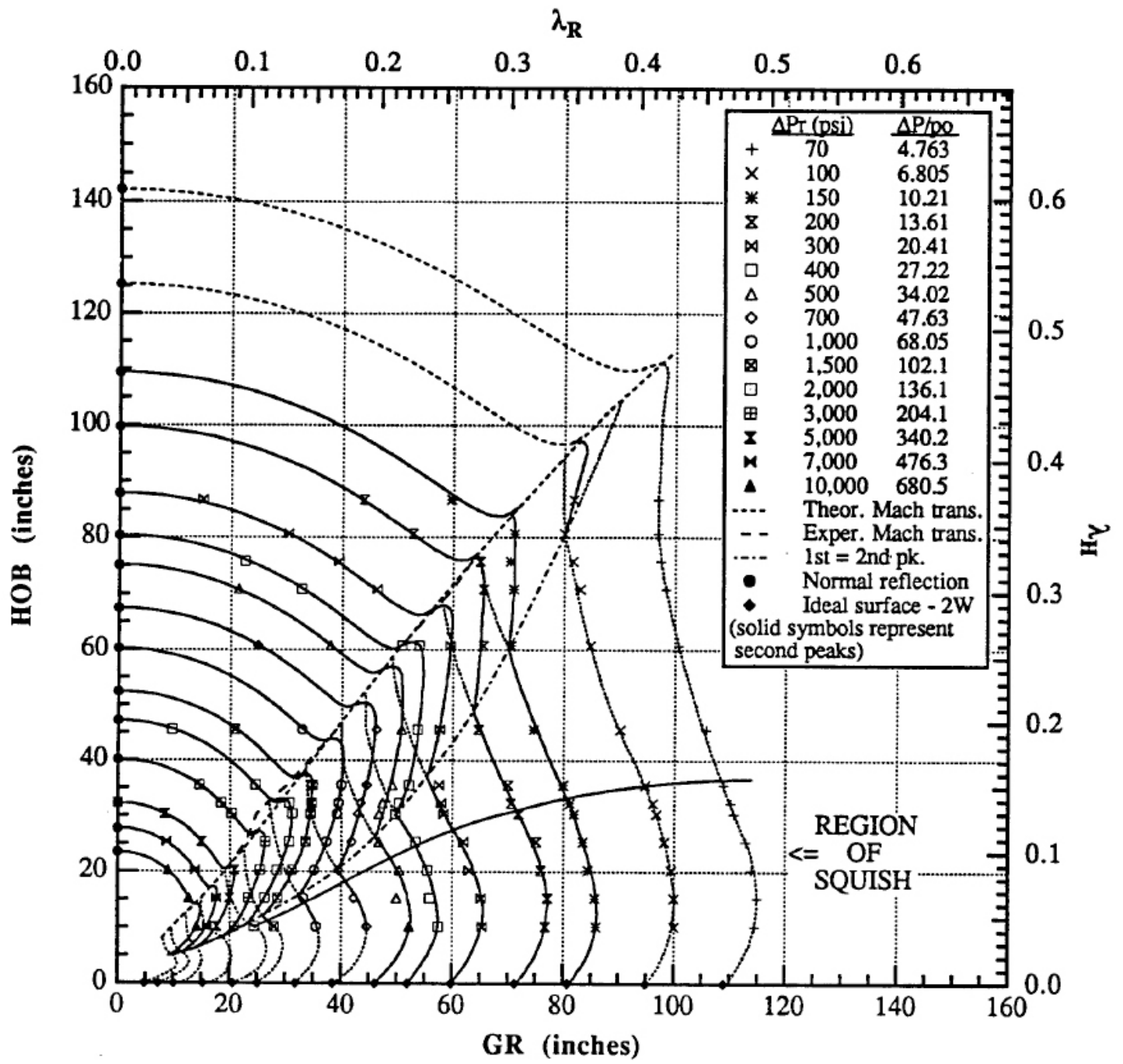

Figure 3-27. Peak overpressure HOB curves from 8-lb PBX-9404 spheres, 70 - 10,000 psi.

${ }^{26}$ H. J. Carpenter, D. J. Michalski, Eight-lb PBX9404 HOB Experiments, Volume 1: Experimental Description and Data Analysis, DNA-TR-94-129-V1, Defense Nuclear Agency, 1995. 
Problem 7. Height of Burst Curves

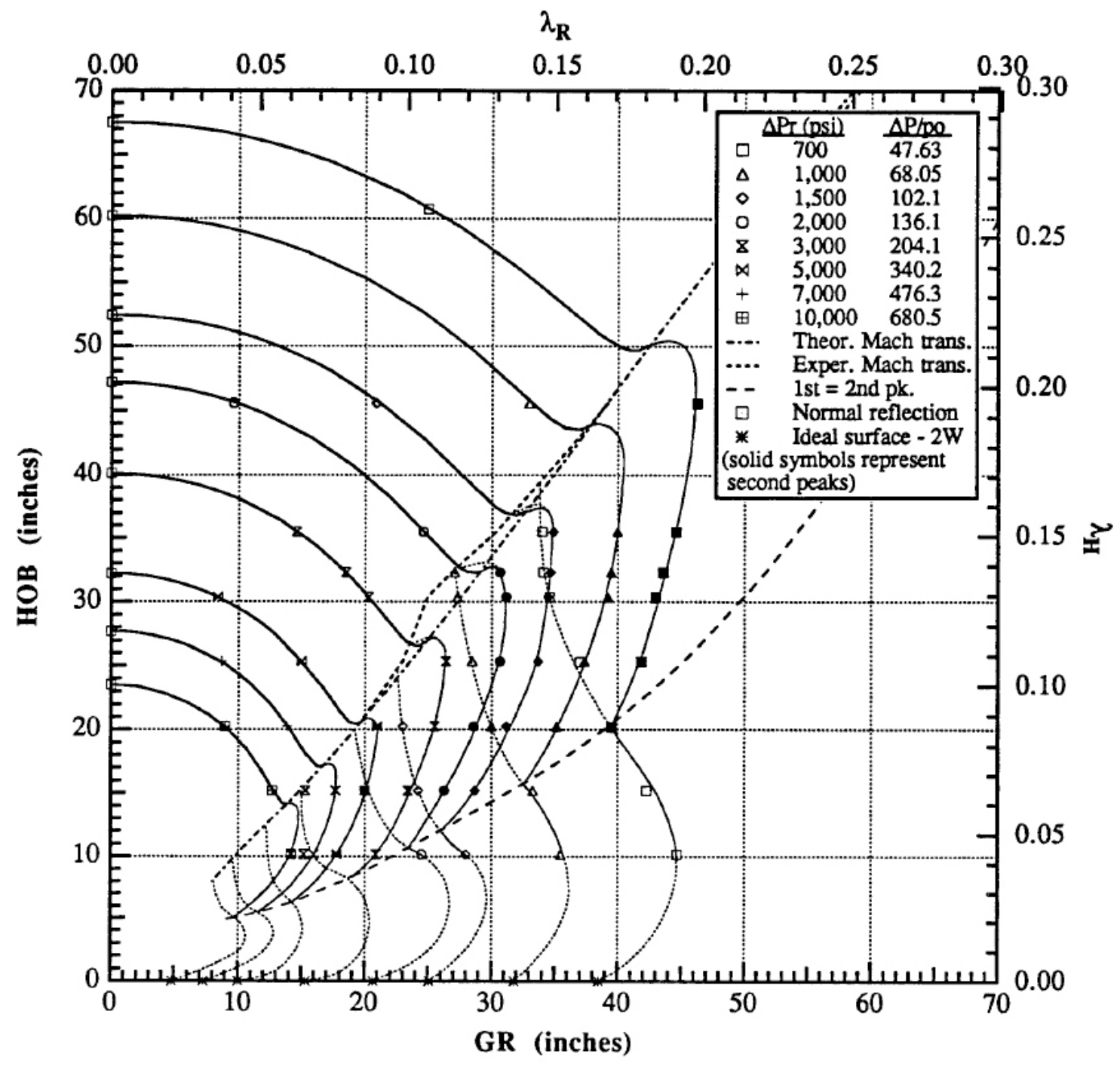

Figure 3-29. First and second peak overpressure HOB curves from 8-lb PBX-9404 spheres, 700 - 10,000 psi. 
7.3 Nuclear HOB Curves for Ideal Surfaces ${ }^{22}$

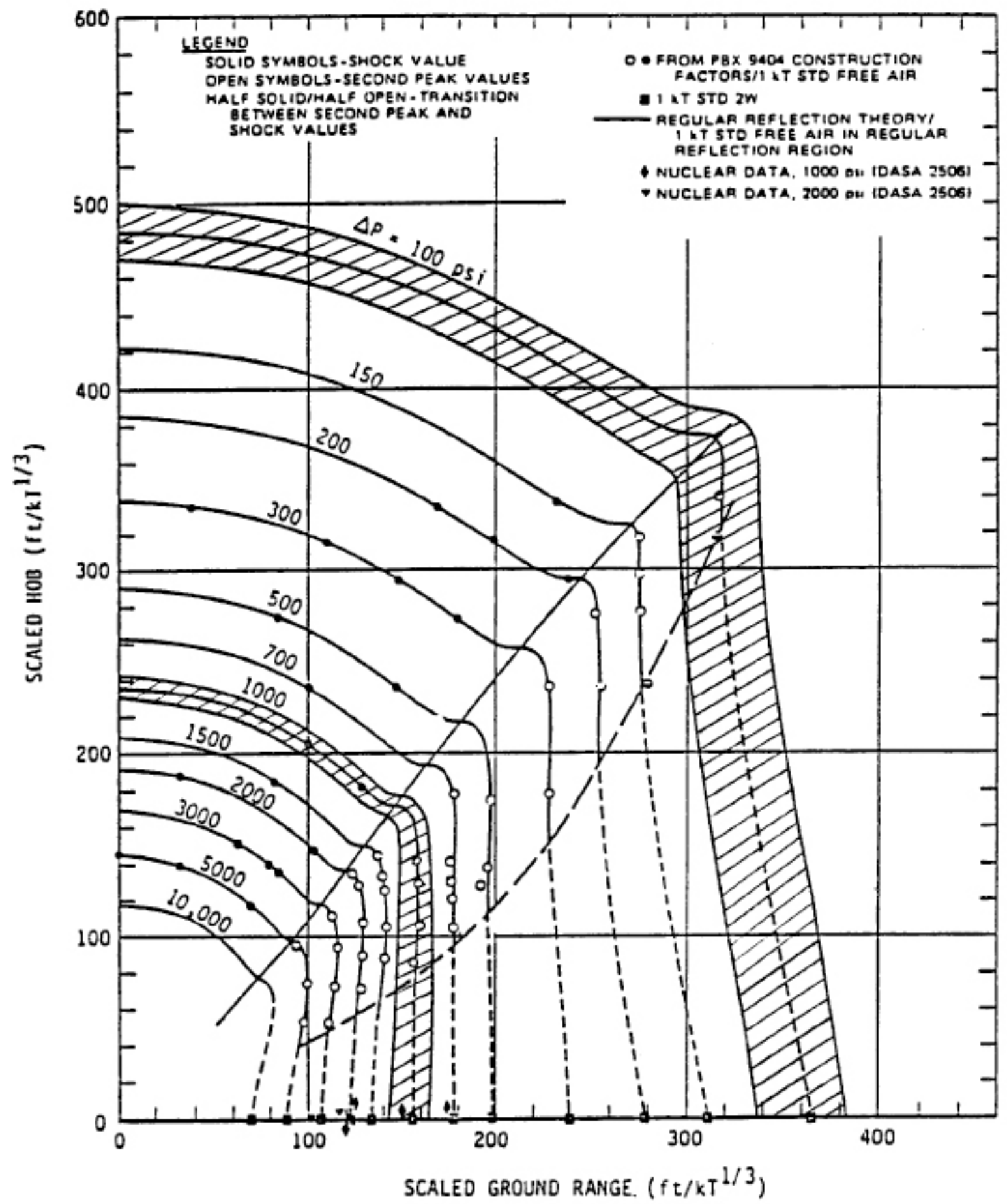

Figure 4-1. Ideal surface peak overpressure HOB curves. 


\section{Problem 7. Height of Burst Curves}

\section{References}

[1] S. Glasstone (Ed.) The Effects of Nuclear Weapons, US Atomic Energy Commission, 1962, $730 \mathrm{pp}$.

[2] P. J. Dolan, Editor, Capabilities of Nuclear Weapons, DNA EM-1 Part I: Phenomenology, Defense Nuclear Agency, Washington, DC 1972 (revised 1 August 1981).

[3] Fred Sauer, A Guide to the NWETI Nuclear Airblast Record Inventory, Vol. 1, 2, Technical Note DTRIAC SR-11-003, August 2011.

[4] H. J. Carpenter, D. J. Michalski, Eight-lb PBX9404 HOB Experiments, Vol. 1: Experimental Description and Data Analysis, DNA-TR-94-129-V1, Defense Nuclear Agency, 1995.

[5] Reichenbach, H. \& Kuhl, A. (1991) HOB curves for 0.5-g NP charges, DNA-TR-90-223, Defense Nuclear Agency, Alexandria, VA, 38 pp. 


\section{Summary and Conclusions}

\section{Radiation-Hydrodynamic Wave}

If one considers explosion effects close to a nuclear weapon $\left(R \leq 8 \mathrm{~m} / K T^{1 / 3}, t \leq 0.1 \mathrm{~ms} / K T^{1 / 3}\right)$, the flow field must be calculated with a radiation hydrodynamics code. Simulations of a free-air burst should be performed and compared with Brode's solution based on a radiation diffusion model (RM-2248).

\section{Blast Waves from Nuclear Explosions}

The hydrodynamic range of the explosion starts when the bomb case shock overtakes the radiation diffusion front $\left(R=8 \mathrm{~m} / K T^{1 / 3}\right.$ and $\left.t=0.1 \mathrm{~ms} / K T^{1 / 3}\right)$. At this point, the blast wave can be calculated with a gasdynamics code ${ }^{27}$. The computational grid can be initialized by the point source similarity solution applied at a convenient point along the free air curve. The domain must be initialized by a realistic atmospheric structure (U.S. Standard 1962 Atmosphere, or atmosphere measured on a specific test event). Results presented in Problem 1 can be used to check the accuracy of numerical simulations of unconfined nuclear explosions; the free air curve serves as the standard measure.

\section{Blast Wave Reflections from Ideal Surfaces}

The ability of a code to calculate flow fields behind reflected shocks can be checked by performing numerical simulations of shock reflections from wedges. Three cases were proposed (corresponding to regular reflection, Mach reflection and double-Mach reflection), but there are 12 other cases available. The ability of a code to accurately predict reflected blast wave pressures in the high-pressure regime can be verified by numerical simulations of the 8-lb charge experiments of Carpenter (DNA-TR-94-129). One case was proposed $(H O B=51.66 \mathrm{~cm})$, but data from 12 other heights of burst are available.

\section{Blast Wave Reflections from Ground Surfaces}

The principal difference between blast waves from nuclear explosions and blast waves from HE charges is the existence of the Precursor Regime. Thermal radiation from the nuclear fireball creates a hot thermal layer on the ground surface. The shock front runs faster in the thermal layer, leading to a precursor shock structure with an embedded wall jet. This wall jet scours dust from the surface, forming a turbulent wall jet flow. The stagnation pressure in the wall jet can be an order of magnitude larger than the corresponding ideal surface case (see Fig. 4 of the Introduction). Based on experimental data described in EM-1, the precursor regime is: $H O B<\sim 700 \mathrm{ft}^{1 / 3}$ and $G R<\sim 1100 \mathrm{ft} / K T^{1 / 3}$. To compute the flow field above the surface in the precursor regime, one must model the thermal layer, turbulent wall jet and dusty boundary layer flow; the models described in Problem 3 (with the iLES model of turbulence) are recommended. If one is outside the precursor regime (i.e., $H O B>700 \mathrm{ft} / K T^{1 / 3}$ or $G R>1100 \mathrm{ft} / K T^{1 / 3}$ ), one can treat the surface as ideal and model it with gasdynamic code simulations.

\footnotetext{
${ }^{27}$ As the fireball temperature drops due to adiabatic expansion, internal energy re-distribution occurs by convective flow, which is faster than radiation diffusion, and a gasdynamic model is adequate.
} 


\section{Summary and Conclusions}

\section{Spherical Mixing Layers}

The turbulence fields have been studied by iLES simulations of turbulent combustion clouds. Taking advantage of the point symmetry of the problem, the flow fields were azimuthally averaged to extract the mean and rms profiles of thermodynamic and kinematic variables, the mixing layer profiles and the evolution of the turbulent kinetic energy spectra. These results can be used to construct models of spherical mixing layers in explosions. And they can be used to check the accuracy of existing turbulence models $(k-\varepsilon$, etc.) to predict turbulent mixing layers in explosions.

\section{Kelvin-Helmholtz Mixing Layers}

To check the ability of hydro codes to simulate slip lines and shear layers embedded in explosions, four free shear layer problems were proposed. These correspond to the seminal experiments of Brown \& Roshko (1974) and Oster \& Wignanski (1982). Such flows are dominated by the rotational velocity component whose simulation can be problematic for Lagrangian-based numerical algorithms. Both two-dimensional and three-dimensional simulations are recommended.

\section{Wall Mixing Layers}

For explosions over ground surfaces, one must model the entrainment and mixing of the dust from the surface. An iLES approach was recommended in Problem 6-whereby the dust is entrained and transported by the rotational structures of the turbulent boundary layer flow. The ability to model the turbulent boundary layer flow is crucial to predicting loads on above ground structures-especially in the precursor regime.

\section{HOB Curves}

Height-of-burst curves are often used in targeting applications, for example, to select the HOB to maximize the damage radius for a selected overpressure. If one is interested in the high-pressure (precursor) regime, one needs HOB curves based on stagnation pressure impulse (which do not now exist). If one is interested in the low-pressure regime ( $\left.\Delta p_{s}<15 \mathrm{psi}\right)$, HOB curves for ideal surfaces are sufficient.

\section{Parametric Studies}

Structural response of above ground structures is proportional to the stagnation pressure impulse. To compute loads in the precursor regime, one needs the stagnation pressure histories as a function of: ( $i$ ) height above the surface, (ii) ground range and (iii) height of burst. Such flow fields can be computed with the models described in Problem 3. A computational matrix is suggested in Table 1. Results can be checked against data from nuclear test events. The flow fields can also be used to initialize numerical simulations of dust clouds from nuclear explosions, to be performed with our low-Mach number AMR code. 


\section{Summary and Conclusions}

Table 1. Computational Matrix for Simulating Nuclear Events

\begin{tabular}{|c|l|c|c|}
\hline Case & \multicolumn{1}{|c|}{ US Test Events } & $\begin{array}{c}\text { HOB } \\
(f t)\end{array}$ & $\begin{array}{c}\text { SHOB } \\
\left(f t / K T^{1 / 3}\right)\end{array}$ \\
\hline 1 & Small Boy/Little Feller & surface & 0 \\
\hline 2 & Trinity (19 KT) & $100 \mathrm{~T}$ & 37 \\
\hline 3 & UPSHOT KNOTHOLE-Simon (43 KT) & $300 \mathrm{~T}$ & 81 \\
\hline 3 & UPSHOT KNOTHOLE-Badger (23 KT) & $300 \mathrm{~T}$ & 106 \\
\hline 4 & TEAPOT-Met (22 KT) & $300 \mathrm{~T}$ & 137 \\
\hline 5 & PLUMBBOB-Priscilla (37 KT) & $700 \mathrm{~B}$ & 200 \\
\hline 6 & Tsar Bomba (58 MT) 10/30/61 & 12,000 & 312 \\
\hline 7 & UPSHOT KNOTHOLE-Climax (61 KT) & 1,334 & 322 \\
\hline 8 & PLUMBBOB-Hood (74 KT) & 1,500 & 340 \\
\hline 9 & PLUMBBOB-Stokes (19 KT) & 1,500 & 533 \\
\hline 10 & Nagasaki ( 22 KT) & 1,640 & 585 \\
\hline 11 & PLUMBBOB-Charleston (12 KT) & 1,500 & 621 \\
\hline 12 & UPSHOT KNOTHOLE-Encore (27 KT) & 2,425 & 766 \\
\hline 13 & Hiroshima ( 12 KT) & 1,850 & 808 \\
\hline 14 & TUMBLER SNAPPER-Charlie (31 KT) & 3,447 & 1040 \\
\hline 15 & UPSHOT KNOTHOLE-Dixie (11 KT) & 6,020 & 2,564 \\
\hline
\end{tabular}

* Samuel Glasstone, Editor, The Effects of Nuclear Weapons, U. S. AEC, 1962, 730 pp.

$\mathrm{T}=$ tower shot, $\mathrm{B}=$ balloon shot 


\section{Recommendations}

\section{Test Problems}

Problems 1-6 should be added to the official suite of test problems used to check hydro-codes developed in A and B Programs.

\section{Radiation-Hydrodynamic Wave}

The radiation-hydrodynamic wave from a nuclear charge should be computed with LLNL codes. Results should be compared with Brode's numerical simulation (RM-2248).

\section{Reflections from Ideal Surfaces}

The ability of AX Division codes to accurately predict reflections from ideal surfaces should be checked by performing shock reflections from wedges and blast wave reflections from an ideal surface-as described in Problem 2.

\section{Spherical Mixing Layers}

The solution of the turbulent flow field presented in Problem 4 should be used to construct and validate turbulent mixing models for spherical explosion fields.

\section{Shear Layers}

The ability of AX Division hydro codes (and/or turbulence models) to accurately predict turbulent mixing in free shear layers should be verified by simulations of the four cases presented in Problem 5.

\section{Stagnation Pressure HOB Curves}

To define the blast loads in the high-pressure regime, a set of numerical simulations of nuclear explosions over ground surfaces should be performed with the models described in Problem 3 . Heights of burst should include cases 2-10 identified in Table 1. The flow fields will be analyzed to produce stagnation pressure impulses as a function of ground range, height above the surface and HOB. 


\section{PROPOSALS}

\section{Proposal 1: \\ Modeling Spherical Mixing in Explosion Fields}

The turbulence fields in spherical combustion clouds have been studied by iLES simulations of the explosion of a spherical TNT charge (Problem 4). Taking advantage of the point symmetry of the problem, the flow fields were azimuthally averaged to extract the mean and rms profiles of thermodynamic and kinematic variables, the mixing layer profiles and the evolution of the turbulent kinetic energy and its spectrum. These results will be used to construct models of spherical mixing layers in explosions. And they can be used to check the accuracy of existing turbulence models $(k-\varepsilon$, etc.) to predict turbulent mixing layers in explosions. 


\section{PROPOSALS}




\section{PROPOSALS}

\section{Proposal 2: \\ Parametric Studies of Turbulent Mixing in Nuclear Explosions over Ground Surfaces}

Numerical simulations of the flow field created by nuclear explosions over ground surfaces will be performed with our AMR explosion code (AMR-EC). The code uses the iLES (implicit Large-Eddy Simulation) approach to capture the energy-bearing scales of turbulence on the computational grid, so no turbulence modeling is needed. Adaptive Mesh Refinement (AMR) is used to follow the evolution of turbulent mixing regions in the flow. The code contains empirical models of the thermal layer and fluidized bed, needed to model the turbulent wall jet and dusty boundary layer flow from first principles (i.e., vorticity in the boundary layer entrains dust from the fluidized bed and distributes it throughout the flow via turbulent mixing). Eleven heights of burst in the precursor regime will be studied:

- $H O B=0 \mathrm{ft} / K T^{1 / 3}$ (Small Boy/Little Feller)

- $H O B=37 \mathrm{ft} / K T^{1 / 3}$ (Event Trinity)

- $H O B=81 \mathrm{ft} / K T^{1 / 3}$ (UPSHOT KNOTHOE-Simon)

- $H O B=106 \mathrm{ft} / \mathrm{KT}^{1 / 3}$ (UPSHOT KNOTHOLE-Badger)

- $H O B=137 \mathrm{ft} / \mathrm{K} T^{1 / 3}$ (TEAPOT-Met)

- $H O B=200 \mathrm{ft} / \mathrm{KT}^{1 / 3}$ (PLUMBBOB-Priscilla)

- $H O B=322 \mathrm{ft} / \mathrm{KT}^{1 / 3}$ (UPSHOT KNOTHOE-Climax)

- $H O B=533 f t / K T^{1 / 3}$ (PLUMBBOB-Stokes)

- $H O B=621 \mathrm{ft} / \mathrm{KT}^{1 / 3}$ (PLUMBBOB-Charleston)

- $H O B=766 f t / K T^{1 / 3}$ (UPSHOT KNOTHOE-Encore)

- $H O B=1040 \mathrm{ft} / K T^{1 / 3}$ (TUMBLER SNAPPER-Charlie)

The results will be analyzed to determine the stagnation pressure history and positive phase impulse as a function of: (i) height above the surface, (ii) ground range and (iii) height of burst. Results can be checked against data from nuclear test events. The flow fields can also be used to initialize numerical simulations of dust clouds from nuclear explosions, to be performed with our low-Mach number AMR code. 


\section{PROPOSALS}




\section{PROPOSALS}

\section{Proposal 3: \\ V\&V of Shock Hydrodynamics Codes}

\section{Radiation-Hydrodynamic Wave (V\&V)}

The radiation-hydrodynamic wave from a nuclear charge should be computed with LLNL codes. Results should be compared with Brode's numerical simulation (RM-2248).

\section{Reflections from Ideal Surfaces $(V \& V)$}

The ability of AX Division codes to accurately predict reflections from ideal surfaces should be checked by performing shock reflections from wedges and blast wave reflections from an ideal surface, as described in Problem 2.

\section{Kelvin-Helmholtz Mixing Layers}

To check the ability of hydro codes to simulate shear layers embedded in explosions, four free shear layer problems were proposed. These correspond to the seminal experiments of Brown \& Roshko (1974) and Oster \& Wignanski (1982). Such flows are dominated by the rotational velocity component whose simulation can be problematic for Lagrangian-based numerical algorithms. Both two-dimensional and three-dimensional simulations are recommended. 


\section{PROPOSALS}




\section{References}

\section{Introduction}

[1] Samuel Glasstone (Editor), Effects of Nuclear Weapons, US Atomic Energy Commission, April 1962, 730 pp.

[2] P. J. Dolan, Editor, Capabilities of Nuclear Weapons, DNA EM-1 Part I: Phenomenology, Defense Nuclear Agency, Washington, DC 1972 (revised 1 August 1981).

[3]A. L. Kuhl, Airblast Effects from Nuclear Explosions over Real Surfaces, DNUG Meeting, April 2012 (in press).

[4] A. L. Kuhl, P. Wang, Nuclear Explosion at 500 feet above a Ground Surface, NECDC 2012 Proceedings (in press)

\section{Problem 1: Blast Waves from Nuclear Charges}

[1] H. L. Brode, Numerical solutions of spherical blast waves, J. App. Phys. 16 (6) 1955, pp. 766-775. (Evolution of blast wave from point-source solution: 2 kilo-bars to 0.1 bar regime)

[2] H. L. Brode, Theoretical Description of the Blast and Fireball for a Sea-Level Megaton Explosion, RM-2248, Rand Corp. 1959, 147 pp.

[3] H. L. Brode, Theoretical Description of the Blast and Fireball for a Sea-Level Kiloton Explosion, RM-2246, 1966 (FOUO).

[4] H. L. Brode, Review of Nuclear Weapons Effects, Annual Reviews of Nuclear Science vol. 18, 1968, pp. 153-202.

[5] S. Glasstone (Ed.) The Effects of Nuclear Weapons, US Atomic Energy Commission, 1962, $730 \mathrm{pp}$.

[6] F. R. Gilmore, Equilibrium Composition and Thermodynamic Properties of Air to 24,000 K, Rand Corp. Report RM-1543, 1955, 68 pp; also see DASA 1971-1 THERMAL RADIATION PHENOMENA, J. L. Magee and H. Aroeste, Eds. 3-27-67-1 Vol. 1, May 1967, 333 pp. (vid. esp. Chapter 1: The Equilibrium Thermodynamic Properties of High Temperature Air by F. R. Gilmore).

[7] H. L. Brode, Blast wave from a spherical charge, Physics of Fluids 2 (2) 1959, 217-229; also Calculation of the Blast Wave from a Spherical Charge of TNT, Rand Corp. Report \# RM1965, 1957, 1-68.

[8] R. K. M. Landshoff, Ed., Thermal Radiation Phenomena; Volume 5: H. L. Brode and R. K. M. Landshoff Radiation Hydrodynamics of High Temperature Air, DASA 1917-5, Lockheed Missiles and Space Company, 1967, 172 pp. (vid. esp. Chapter 2: Physics of Fireballs, pp. 36-58, one kiloton at sea level conditions).

[9] H. L. Brode, Gasdynamic motion with radiation: a general numerical method, Astronautica ACTA vol. 14 (5), 1969, pp. 443-444.

[10] M. Dubin, N. Sissenwine, H. Wexler, Co-chairmen, US Standard Atmosphere 1962, US Government Printing Office, Washington, DC 1962, 278 pp. (produced by the United States Committee on Extension to the Standard Atmosphere: COESA)

[11] A. K. Oppenheim, A. L. Kuhl, E. A. Lundstrom, M. M. Kamel, A parametric study of selfsimilar blast waves, J. Fluid Mech. 52(4), 1972, pp. 657-682.

[12] Taylor, G. I., "The Formation of a Blast Wave by a Very Intense Explosion, Part 1: Theoretical Discussion," first published in British Report RC-210, 1941; revised version in Proc. Royal Society of London Series A, Vol. 201, 1950, pp. 159-174. 


\section{References}

[13] Taylor, G. I., "The Formation of a Blast Wave by a Very Intense Explosion, Part 2: The Atomic Explosion of 1945" Proc. Royal Society of London Series A, Vol. 201, 1950, pp 175186.

[14] von Neumann, J. "The Point Source Solution," first published in NDRC Div. B. Report AM-9, 1941; then in Shock Hydrodynamics and Blast Waves, Bethe, H. A., ed., AECD-2860, 1944; revised version in Blast Waves, Bethe, H. A., ed. , Los Alamos Scientific Laboratory Report LA-2000, 1947, pp. 27-55; reprinted in John von Neumann Collected Works, Taub, A. H, ed., Vol. VI, Pergamon Press, New York 1963, pp. 219-237.

[15] von Neumann, J., and Goldstine, H., "Blast Wave Calculation," Comm. Pure and Applied Mathematics Vol. 8, 1955, pp. 327-353; reprinted in John von Neumann Collected Works, Taub, A. M., ed., Vol. VI, Pergamon Press , New York, 1963 , pp . 386-412.

[16] Sedov, L. I., "The Motion of Air due to an Intense Explosion" Doklady AN SSSR, Vol. 52, No. 1, 1946, pp 17-20.

[17] Sedov, L. I., "Rasprostraneniya sil'nykh vzryvnykh voln" (The Propagation of Intense Explosion Waves), Prikladnaya Matematika i Mekhanika, Vol. 10, No 2, 1946, pp. 241-250.

[18] Stanyukovich. K. P., "Application of Particular Solutions for Equations of Gas Dynamics to the Study of Blast and Shock Waves," Report of the Academy of Science of the USSR, Vol. 52, No. 7, 1946.

[19] Stanyukovich. K. P., Unsteady Motion of Continuous Media, Gostekhizdat, Moscow, 1955; English translation. Holt, M., ed., Pergamon Press, New York, 1960, 745 pp.

[20] P. W. Bridgeman, Dimensional Analysis, Yale University Press, 1922, 113 pp.

[21] Sedov, L. I., Similarity and Dimensional Method in Mechanics, Friedman, M., translator, Holt, H., ed., 4th Printing, Academic Press, New York, 1959, (vid. sp. "Chapter IV: Onedimensional Unsteady Motion of a Gas"); orginially published as Methody teorii razmernostey I teorii podobiyav mekhanike (Methods of Dimensional Theory and Similarity Theory in Mechanics), Moscow-Leningrad, Gostekhizdat, 1944, 136 pp.

[22] P. J. Dolan, Editor, Capabilities of Nuclear Weapons, DNA EM-1 Part I: Phenomenology, Defense Nuclear Agency, Washington, DC 1972 (revised 1 August 1981).

[23] A. L. Kuhl, P. Wang, Nuclear Explosion at 500 feet above a Ground Surface, NECDC 2012 Proceedings (in press)

\section{Problem 2: Blast Reflections from Ideal Surfaces}

[1] H. M. Glaz, P. Colella, I. I. Glass, and R. L. Deschambault, A Detailed Numerical, Graphical and Experimental Study of Shock Wave Reflections, LBL-20033, Lawrence Berkeley Laboratory, 1985, $380 \mathrm{pp}$.

[2] H. M. Glaz, P. Colella, I. I. Glass, R. L. Deschambault (1985a) A numerical study of oblique shock-wave reflections with experimental comparisons. Proc. R. Soc. London, Ser. A, 398, pp. 117-140.

[3] P. Colella, R. E. Ferguson, H. M. Glaz, A. L. Kuhl, Mach reflection from an HE-driven blast wave, Dynamics of Explosions, AIAA Progress in Astronautics and Aeronautics, vol. 106, 1985, pp. 388-421.

[4] H. J. Carpenter, D. J. Michalski, Eight-lb PBX9404 HOB Experiments, Vol. 1: Experimental Description and Data Analysis, DNA-TR-94-129-V1, Defense Nuclear Agency, 1995. 


\section{References}

\section{Problem 3: Blast Reflections from Ground Surfaces}

[1] Kuhl, A. L. (1993) Turbulent wall jet in a blast wave precursor (plenary lecture), 1993 Japanese National Symposium on Shock Wave Phenomena (K. Takayama Ed.), Tohoku Print, Sendai, Japan. Also published as UCRL-JC-112713, Lawrence Livermore National Laboratory, Livermore, CA, $40 \mathrm{pp}$.

\section{Problem 4: Spherical Mixing Layers}

[1] A. L. Kuhl, J. B. Bell, V. E. Beckner, K. Balakrishnan, Spherical combustion clouds in explosions, Shock Waves 2012 (in press).

\section{Problem 5: KH Mixing Layers}

[1] A. L. Kuhl, K.Y. Chien, R. E. Ferguson, H. M. Glaz, P. Colella, Inviscid dynamics of unstable shear layers, RDA-TR-161604-006, August 1988, 94 pp.

[2] K-Y. Chien, R. E. Ferguson, A. L. Kuhl, H. M. Glaz, P. Colella, Inviscid dynamics of twodimensional shear layers, Int. J. Computational Fluid Dynamics, 1995, 5 (1-2), p. 59-80.

[3] G. L. Brown, A. Roshko, On density effects and large structures in turbulent mixing layers, $J$. Fluid Mechanics 64, 1974, pp. 775-816.

[4] D. Oster, I. Wygnanski, The forced mixing layer between parallel streams, J. Fluid Mechanics 123, 1982, pp. 91-130.

\section{Problem 6: Shock-induced Wall Mixing Layers}

[1] Kuhl, A. L., Chien, K.-Y., Ferguson, R. E., Collins, J. P., Glaz, H. M., \& Colella, P. (1989) Simulation of a turbulent dusty boundary layer behind a shock. In Proceedings of the Seventeenth Int. Symposium of Shock Waves and Shock Tubes, Lehigh University, Bethlehem, PA, 17-21 July. Published in Current Topics in Shock Waves (ed. Y. W. Kim), AIP Conference Proceedings 208, American Institute of Physics, New York, NY, pp. 762-769, 1990 (ISBN 0-88318-776-0) Also published as RDA-TR-0263229004-001, R \& D Associates, Los Angeles, CA.

[2] Kuhl, A. L., Reichenbach, H. \& Ferguson, R. E., (1991) Shock interactions with a dense-gas wall layer, presented at the Eighteenth Int. Symposium on Shock Waves, 21-26 July 1991, Sendai, Japan. Published in Shock Waves: Proceedings of Eighteenth Int. Symposium on Shock Waves (K. Takayama Ed.) Springer, Berlin pp. 159-166.

\section{Problem 7: Height of Burst Curves}

[1] S. Glasstone (Ed.) The Effects of Nuclear Weapons, US Atomic Energy Commission, 1962, $730 \mathrm{pp}$.

[2] P. J. Dolan, Editor, Capabilities of Nuclear Weapons, DNA EM-1 Part I: Phenomenology, Defense Nuclear Agency, Washington, DC 1972 (revised 1 August 1981).

[3] Fred Sauer, A Guide to the NWETI Nuclear Airblast Record Inventory, Vol. 1, 2, Technical Note DTRIAC SR-11-003, August 2011.

[4] H. J. Carpenter, D. J. Michalski, Eight-lb PBX9404 HOB Experiments, Vol. 1: Experimental Description and Data Analysis, DNA-TR-94-129-V1, Defense Nuclear Agency, 1995.

[5] Reichenbach, H. \& Kuhl, A. (1991) HOB curves for 0.5-g NP charges, DNA-TR-90-223, Defense Nuclear Agency, Alexandria, VA, 38 pp. 